\title{
INSTABILIDADE LATERAL DAS VIGAS PRÉ-MOLDADAS EM REGIME DE SERVIÇO E DURANTE A FASE TRANSITÓRIA
}

MARIA CRISTINA VIDIGAL DE LIMA

Dissertação apresentada à Escola de Engenharia de São Carlos, da Universidade de São Paulo, como parte dos requisitos necessários para a obtenção do Título de " Mestre em Engenharia de Estruturas".

ORIENTADOR: Mounir Khalil El Debs

São Carlos

1995 


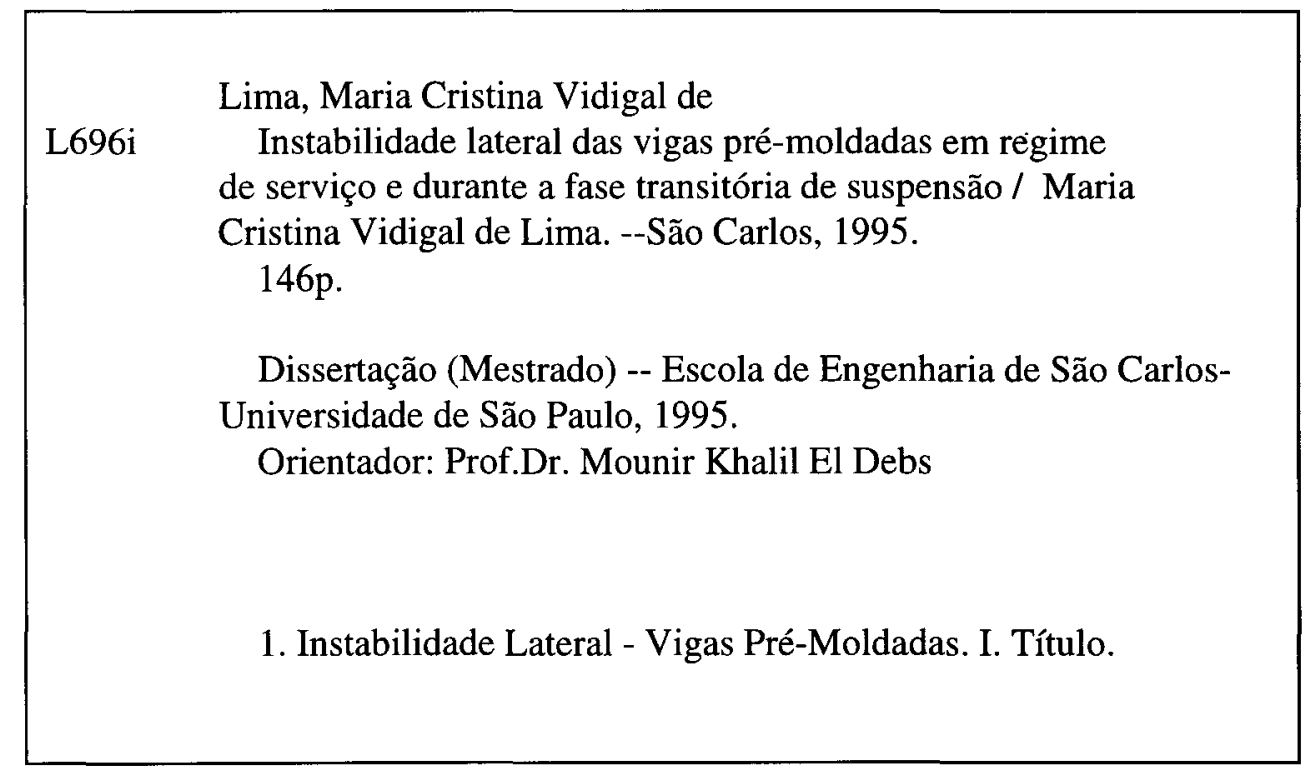


Aos meus amados pais, pela participação especial e carinhosa em minha vida. 


\section{AGRADECIMENTOS}

À Deus, pela crença e pela importante oportunidade.

Ao meu orientador Mounir Khalil El Debs pela paciência e valiosa colaboração.

Ao professor Munir Rachid, co-orientador deste trabalho, pelos minutos diários de atenção e pelos exemplos constantes de solidariedade.

À Rosi Aparecida Jordão Rodrigues e Maria Nadir Minatel, pela companhia afetuosa e pelas incontáveis horas de dedicação.

Ao Francisco Carlos G. Brito, Sylvia Helena Morette Villani, Marta Regina Couto Faria, Rui Casale, Antonio Valdair Carneiro e Roberto dos Santos Junior pela ajuda, muito preciosa, para a realização deste trabalho.

Ao $\mathrm{CNPq}$, pelo apoio financeiro que possibilitou a realização desta dissertação.

Aos colegas do departamento, pela amizade.

Às amigas, Larissa, Aninha, Paula, Regina e Ronisi, pelo tempo de convivência saudável, pela simpatia, pela colaboração constante e pela grande e bela amizade.

Aos meus irmãos, Dri, Ciça, Rodrigo e Edu, pelo carinho à distância.

À tia Neusa e minhas queridas avós, pela grandeza de suas intenções. 


\section{SUMÁRIO}

LISTA DE QUADROS $\ldots \ldots \ldots \ldots \ldots \ldots \ldots \ldots \ldots \ldots \ldots \ldots \ldots \ldots$

LISTA DE TABELAS $\ldots \ldots \ldots \ldots \ldots \ldots \ldots \ldots \ldots \ldots \ldots \ldots \ldots \ldots \ldots \ldots$

LISTA DE FIGURAS $\ldots \ldots \ldots \ldots \ldots \ldots \ldots \ldots \ldots \ldots \ldots \ldots \ldots \ldots \ldots \ldots$ ii

LISTA DE SÍMBOLOS $\ldots \ldots \ldots \ldots \ldots \ldots \ldots \ldots \ldots \ldots \ldots \ldots \ldots$

1 INTRODUÇÃO $\ldots \ldots \ldots \ldots \ldots \ldots \ldots \ldots \ldots \ldots \ldots \ldots \ldots \ldots \ldots \ldots \ldots$

1.1 Revisão bibliográfica $\ldots \ldots \ldots \ldots \ldots \ldots \ldots \ldots \ldots \ldots \ldots \ldots \ldots \ldots \ldots$

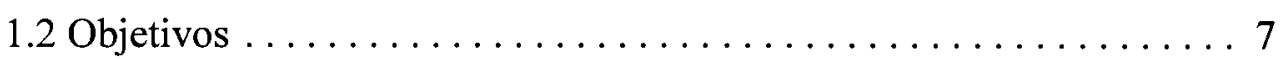

1.3 Apresentação do trabalho $\ldots \ldots \ldots \ldots \ldots \ldots \ldots \ldots \ldots$

2 INSTABILIDADE LATERAL DE VIGAS DURANTE A FASE DE

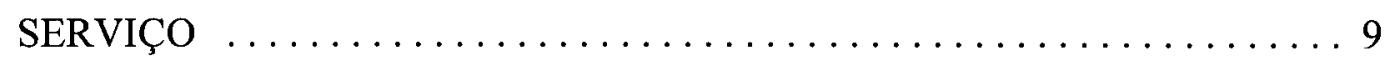

2.1 Preliminares $\ldots \ldots \ldots \ldots \ldots \ldots \ldots \ldots \ldots \ldots \ldots \ldots$

2.2 Equações diferenciais regentes da instabilidade lateral para algumas seções típicas $\ldots \ldots \ldots \ldots \ldots \ldots \ldots \ldots \ldots \ldots \ldots \ldots \ldots \ldots \ldots \ldots \ldots \ldots \ldots \ldots$

2.3 Resolução da equação diferencial para vigas retangulares . . . . . . 13

2.3.1 Processo de integração por séries de potência . . . . . 13

2.3.2 Método de Runge-Kutta . . . . . . . . . . . 17

2.4 Instabilidade lateral de vigas protendidas em serviço $\ldots \ldots \ldots 21$

2.4.1 Considerações gerais sobre a energia potencial total . . . 21

2.4 .2 Ação isolada da protensão . . . . . . . . . 23

2.4.3 Ação conjunta do carregamento vertical da força de protensão $\ldots \ldots \ldots \ldots \ldots \ldots \ldots \ldots \ldots \ldots \ldots \ldots \ldots \ldots \ldots \ldots \ldots$

2.5 Instabilidade lateral de vigas em serviço sobre apoios deformáveis . 33

2.5.1 Viga sobre apoios deformáveis à torção . . . . . . 34 
3 INSTABILIDADE LATERAL DE VIGAS DURANTE A FASE

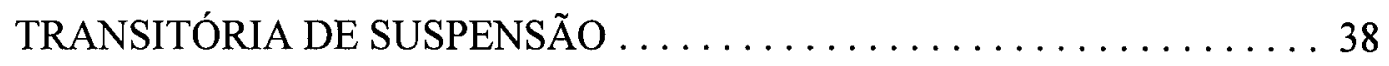

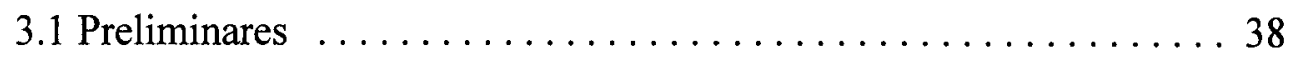

3.2 Estudo da instabilidade utilizando integração por séries de potência . 40

3.2.1 Cabos oblíquos ....................... 40

3.2.2 Cabos retos ........................ 44

3.2.2.1 Suspensão por 2 pontos . . ............ 44

3.2.2.2 Suspensão por 3 pontos . ............. 55

3.2.2.3 Suspensão por 1 ponto $\ldots \ldots \ldots \ldots \ldots \ldots 5$

3.3 vigas protendidas durante a fase transitória

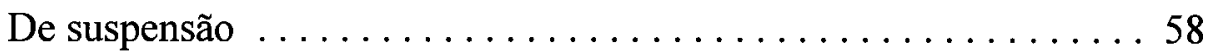

3.3.1 Ação isolada da protensão $\ldots \ldots \ldots \ldots \ldots \ldots \ldots \ldots 58$

3.3.2 ação de eventuais outros

carregamentos distribuídos $\ldots \ldots \ldots \ldots \ldots \ldots 62$

3.4 Aplicação do método de Runge-Kutta em problemas de instabilidade lateral de vigas em suspensão $\ldots \ldots \ldots \ldots 63$

3.4 .1 Introdução $\ldots \ldots \ldots \ldots \ldots \ldots \ldots \ldots \ldots \ldots \ldots \ldots \ldots \ldots \ldots$

3.4.2 Preliminares do método numérico de Runge-Kutta em problemas de auto-valor . . . . . . . . . 65

3.4.3 Viga de seção retangular suspensa por cabos verticais . 69

3.4.4 Viga de seção retangular suspensa por cabos inclinados .72

3.4.5 Viga de seção duplo t simétrico suspensa por cabos

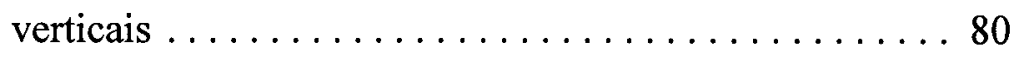

3.4.6 Viga de seção duplo t simétrico suspensa por cabos inclinados $\ldots \ldots \ldots \ldots \ldots \ldots \ldots \ldots . \ldots . \ldots . \ldots$

\section{PROCEDIMENTOS SIMPLIFICADOS PARA DETERMINAÇÃO DO}

FATOR DE SEGURANÇA DURANTE A FASE TRANSITÓRIA . . . . . 87

4.1 Preliminares $\ldots \ldots \ldots \ldots \ldots \ldots \ldots \ldots \ldots \ldots \ldots \ldots \ldots \ldots \ldots \ldots$

4.2 Vigas pré-moldadas de concreto durante fase de içamento $\ldots \ldots 88$

4.3 Vigas pré-moldadas de concreto durante o transporte $\ldots \ldots \ldots 100$ 
4.4 Considerações gerais sobre os fatores de segurança $\ldots \ldots \ldots \ldots 107$

5 EXEMPLOS NUMÉRICOS E ANÁLISES DOS RESULTADOS . . . . . 113

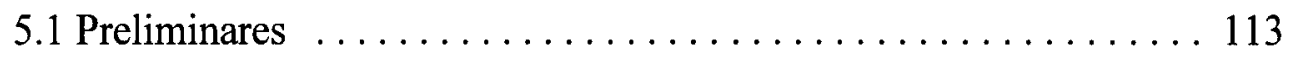

5.2 Viga de seção retangular $\ldots \ldots \ldots \ldots \ldots \ldots \ldots \ldots \ldots \ldots \ldots$

5.2 .1 Fase de serviço $\ldots \ldots \ldots \ldots \ldots \ldots \ldots \ldots \ldots \ldots$

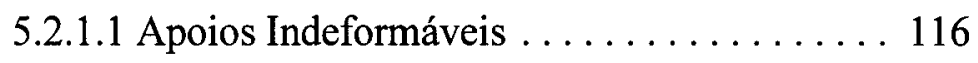

5.2.1.2 Apoios Deformáveis ........... 117

5.2.2 Fase transitória de suspensão $\ldots \ldots \ldots \ldots \ldots \ldots 118$

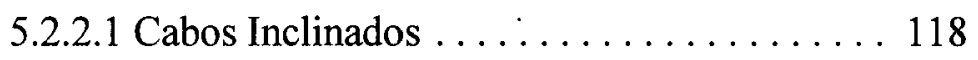

5.2.2.1.1 Séries de potência ......... 119

5.2.2.1.2. Runge-Kutta .......... 120

5.2.2.2 Cabos Retos $\ldots \ldots \ldots \ldots \ldots \ldots \ldots 121$

5.2.2.2.1 Sem balanços ......... 121

5.2.2.2.2 Com balanços .......... 122

5.3 Viga de argamassa armada $\ldots \ldots \ldots \ldots \ldots \ldots \ldots \ldots \ldots \ldots$

5.3 .1 Fase de serviço $\ldots \ldots \ldots \ldots \ldots \ldots \ldots \ldots \ldots \ldots$

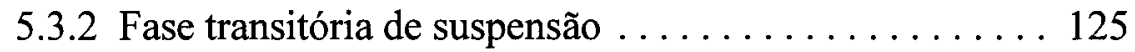

5.3.2.1 Cabos inclinados e Cabos Retos ........ 125

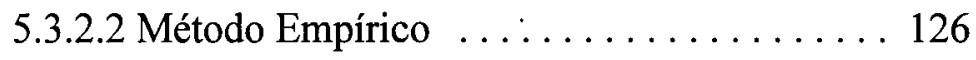

5.4 Viga protendida PCI BT-72 . . . . . . . . . . . 131

5.4 .1 Fase de serviço . . . . . . . . . . . . 132

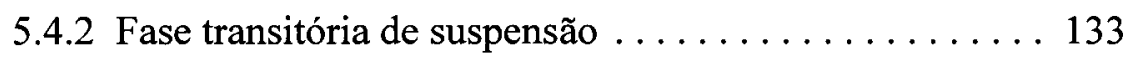

5.4.2.1 Cabos Inclinados $\ldots \ldots \ldots \ldots \ldots \ldots . \ldots 133$

5.4.2.2 Cabos Retos ............... 135

5.4.2.2.1 Sem balanços . . ......... 135

5.4.2.2.2 Com balanços .......... 139

6 CONSIDERAÇÕES FINAIS E CONCLUSÕES $\ldots \ldots \ldots \ldots \ldots \ldots \ldots$ 


\section{LISTA DE QUADROS}

Quadro 2.1 - Equações diferenciais regentes da instabilidade lateral.

$[$ Rachid (1993) ] . . . . . . . . . . . . . . . . . . 12

Quadro 2.2 - Equações diferenciais regentes da instabilidade lateral.

[ CATANIA \& COCCHI (1985) ] . . . . . . . . . . . . 12

Quadro 3.1 - Procedimentos para superposição de efeitos - Sistema de ordem 6 . 78

Quadro 3.2 - Procedimentos para superposição de efeitos - Sistema de ordem $4 \quad .82$

Quadro 3.3 - Procedimentos para superposição de efeitos - Sistema de ordem 8 . 86

Quadro 5.1 - Esquema geral dos casos analisados e os respectivos processos

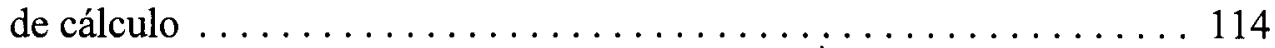




\section{LISTA DE TABELAS}

Tabela 2.1 - Valores de $\mathrm{k}$ relativos ao tipo de apoio.

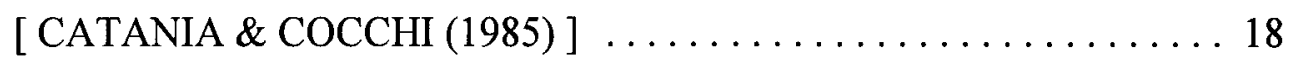

Tabela 3.1 - Valores dos parâmetros k em função de $\bar{d} . ~ \ldots . \ldots . \ldots . \ldots 53$

Tabela 3.2 - Expressões diferenciais de $1^{\mathrm{a}}$ ordem - Sistema de ordem $6 \ldots \ldots$. . 77

Tabela 3.3 - Expressões diferenciais de $1^{\mathrm{a}}$ ordem - Sistema de ordem $4 \ldots \ldots . .82$

Tabela 3.4 - Expressões diferenciais de $1^{\text {a }}$ ordem - Sistema de ordem $8 \ldots \ldots 85$

Tabela 5.1 - Ângulos $\gamma \times$ carga crítica Viga-testeira padrão da ABCI . . . . . 120

Tabela 5.2 - Valores da carga crítica conforme variação de $\overline{\mathrm{d}}$. . . . . . . . . . . 122

Tabela 5.3 - Carga crítica para a variação do ângulo $\gamma$ - Viga de argamassa

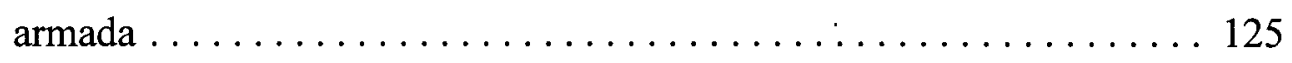

Tabela 5.4 - Valores da carga crítica em função do ângulo $\gamma$ - Viga protendida

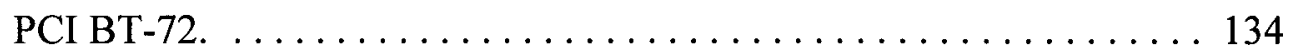




\section{LISTA DE FIGURAS}

Figura 1.1 - Manuseio de uma viga pré-moldada de concreto

[ IMPER \& LASLO (1987) ] . . . . . . . . . . . . . 2

Figura 1.2 - Colapso de uma viga pré-moldada de concreto durante o transporte

[ IMPER \&LASLO (1987) ] . . . . . . . . . . . . . . 3

Figura1.3 - Colapso de uma viga por instabilidade lateral

[ CATANIA \& COCCHI (1985) ] . . . . . . . . . . . . 4

Figura 2.1 - Nomenclatura e sistema de eixos coordenados segundo

CATANIA \& COCCHI $(1985) \ldots \ldots \ldots \ldots \ldots \ldots \ldots \ldots \ldots \ldots$

Figura 2.2 - Viga de seção retangular apoiada sobre garfos. . . . . . . . . 18

Figura 2.3 - Viga protendida de seção duplo T.

[ CATANIA \& COCCHI (1985) ] ................... 24

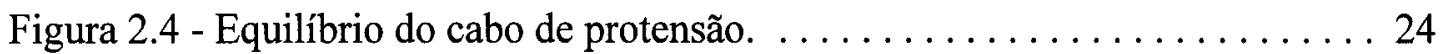

Figura 2.5 - Viga protendida biapoiada sob carregamento vertical.

[ CATANIA \& COCCHI (1985) ] . . . . . . . . . . . 31

Figura 2.6 - Viga apoiada sobre vínculo de garfo deformável à torção. . . . . . . 34

Figura 2.7 - Viga em serviço sobre apoios deformáveis à torção. . . . . . . . 35

Figura 2.8 - Gráfico da função $f(\alpha)$ em função de $\psi \ldots \ldots \ldots \ldots \ldots \ldots \ldots$

Figura 3.1 - Excentricidade $e$. CATANIA \& COCCHI (1985). . . . . . . . 41

Figura 3.2 - Vista em corte da viga deslocada.

[ CATANIA \& COCCHI (1985) ] . . . . . . . . . . . . 42

Figura 3.3 - Gráfico da função $f(\alpha)$ em função de $\psi \ldots \ldots \ldots \ldots \ldots \ldots \ldots$

Figura 3.4 - Viga suspensa por cabos retos com balanços.

[ CATANIA \& COCCHI (1985) ] . . . . . . . . . . . . 45

Figura 3.5 - Gráfico da função $\mathrm{j}(\alpha)$ para $\overline{\mathrm{d}}=1 . \ldots \ldots \ldots \ldots \ldots \ldots \ldots$

Figura 3.6 - Gráfico da função $\mathrm{j}(\alpha)$ para $\overline{\mathrm{d}}=0 . \ldots \ldots \ldots \ldots \ldots \ldots \ldots \ldots \ldots \ldots \ldots \ldots \ldots$ 
Figura 3.7 - Gráfico da função $\mathrm{j}(\alpha)$ para $\overline{\mathrm{d}}=0,5 \ldots \ldots \ldots \ldots \ldots \ldots \ldots \ldots \ldots \ldots \ldots \ldots$

Figura 3.8 - Gráfico da função $\mathrm{j}(\alpha)$ para $\overline{\mathrm{d}}=0,3 \ldots \ldots \ldots \ldots \ldots \ldots \ldots \ldots \ldots$

Figura 3.9 - Gráfico de $\psi$ em função de d. [ CATANIA \& COCCHI (1985) ] . . . 51

Figura 3.10 - Diagrama dos valores de $\mathrm{k}$ e $1 / \mathrm{k}$.

[ CATANIA \& COCCHI (1985) ] .................... 54

Figura 3.11 - Viga suspensa em 3 pontos.[ CATANIA \& COCCHI (1985)] . . 55

Figura 3.12 - Esquema de viga suspensa no vão central.

[ CATANIA \& COCCHI (1985) ] ..................... 56

Figura 3.13 - Gráfico de suspensão em $\mathrm{x}=\ell / 2$.

[ CATANIA \& COCCHI (1985) ] . . . . . . . . . . . . . . . 57

Figura 3.14 - Comparação entre os máximos momentos fletores

para carregamento uniformemente distribuído $\ldots \ldots \ldots \ldots \ldots \ldots . \ldots 57$

Figura 3.15 - Viga protendida em suspensão.

[ CATANIA \& COCCHI (1985) ] . . . . . . . . . . . . . . . . 59

Figura 3.16 - Viga de seção retangular suspensa por cabos verticais. . . . . . . . 69

Figura 3.17 - Viga de seção retangular suspensa por cabos inclinados. . . . . . 72

Figura 3.18 - Equilíbrio da viga em suspensão por cabos inclinados ........ 73

Figura 3.19 - Diagrama para definição da expressão do momento de torção nos apoios. . . . . . . . . . . . . . . . . 76

Figura 3.20 - Viga de seção duplo T simétrico suspensa por cabos verticais. . . . 80

Figura 3.21 - Viga de seção duplo T simétrico suspensa por cabos inclinados. . . 83

Figura 4.1 - Equilíbrio da viga durante a suspensão. [ MAST (1989) ] . . . . . . 89

Figura 4.2 - Relação entre a inércia efetiva e o ângulo $\phi$. [ MAST (1993) ] . . ... 95

Figura 4.3 - Braço do momento aplicado e do momento resistente.

[ MAST (1993) ] ............................... 96

Figura 4.4 - Braço dos momentos x ângulo de inclinação. [MAST (1993) ] . . . 97

Figura 4.5 - Fatores de segurança contra fissuração. [ MAST (1993) ] . . . . . . 97

Figura 4.6 - Viga fissurada para $\phi>\phi_{\max }$ [ MAST (1993) ] . ............. 99

Figura 4.7 - Braço do momento aplicado para apoio na face inferior.

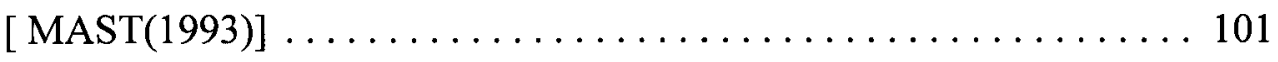

Figura 4.8 - Braço dos momentos $\mathrm{x}$ ângulo de inclinação da viga sobre a 


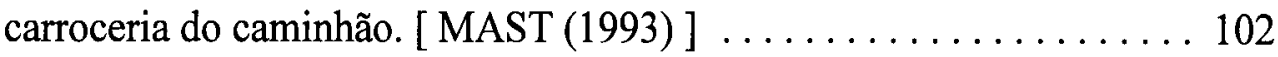

Figura 4.9 - Máximo braço do momento resistente durante o transporte.

[MAST (1993) ] . . . . . . . . . . . . . . . . . 103

Figura 4.10 - Importância da rigidez à rotação da carroceria e da cabine.

[MAST (1993) ] . . . . . . . . . . . . . . . . . . . 104

Figura 4.11 - Situação das cargas no caso da carroceria em posição normal.

[ CUNHA (1994) ] .............................. 105

Figura 4.12 - Situação das cargas para carroceria em posição extendida.

[ CUNHA (1994) ] . . . . . . . . . . . . . . . . . 106

Figura 4.13 - Esquema para a determinação da superelevação da estrada medida no próprio caminhão de transporte. [ CUNHA (1994) ] . . . 106

Figura 4.14 - Efeito dos balanços no fator de segurança em vigas na fase de suspensão. [ MAST (1993)] . . . . . . . . . . . . . . . . 108

Figura 4.15 - Influência da posição dos balanços em vigas protendidas.

[ IMPER \& LASZLO (1987) ] . . . . . . . . . . . . . . . 108

Figura 5.1 - Seção transversal padrão da ABCI. . . . . . . . . . . . . 116

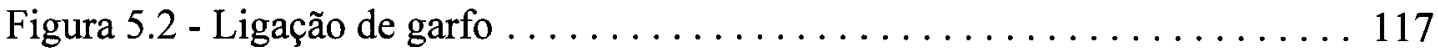

Figura 5.3 - Seção transversal da viga de argamassa armada. . . . . . . . . . 123

Figura 5.4 - Viga suspensa por cabos inclinados presos às extremidades. . . . . 125

Figura 5.5 - Diagrama de momento fletor e equação para máximo momento fletor

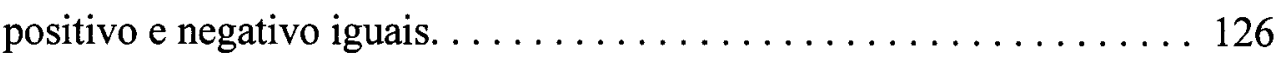

Figura 5.6 - Características geométricas da parábola para suspensão com balanços.

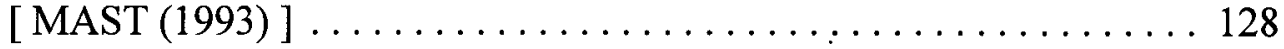

Figura 5.7 - Seção transversal viga PCI BT-72. . . . . . . . . . . . . 131

Figura 5.8 - Disposição do cabo de protensão na viga pré-moldada de

concreto. ............................ 131

Figura 5.9 - Diagrama das tensões normais atuantes na seção do meio do vão para

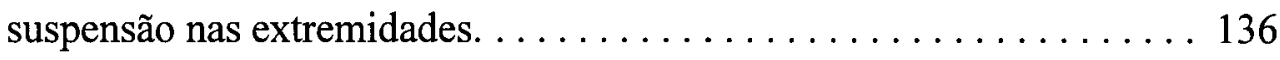

Figura 5.10 - Propriedades da parábola para suspensão nas extremidades. . . . . 136

Figura 5.11 - Diagrama das tensões normais atuantes na seção do meio do vão para suspensão com balanços. 


\section{LISTA DE SÍMBOLOS}

Romanos:

a $\quad$ Metade do vão $\ell$ da viga

$\bar{a} \quad$ Comprimento do balanço

$\mathrm{A}, \mathrm{S} \quad$ Área da seção transversal

$\mathrm{A}_{\mathrm{a}} \quad$ Área da seção transversal do cabo de suspensão

$\mathrm{A}_{\mathrm{i}}^{*}, \mathrm{~A}_{\mathrm{i}} \quad$ Coeficientes genéricos

$\mathrm{B}_{\mathrm{i}} \quad$ Coeficientes genéricos

B Bimomento

$\mathrm{c}_{\mathrm{a}}, \mathrm{c}_{\mathrm{r}} \quad$ Braço do momento aplicado e resistente, respectivamente

CG Centro de gravidade da seção

d Distância do CG ao ponto de fixação dos cabos na suspensão por pontos na face superior da viga

$d^{*} \quad$ Distância do carregamento aplicado em relação ao centro de torção

d Posição do cabo de içamento em relação à seção central de simetria da viga

D Centro de torção

$\mathrm{e}_{0} \quad$ Excentricidade do cabo de protensão em relação à linha do CG

$\mathrm{e}_{\mathrm{i}} \quad$ Excentricidade inicial

$e_{y}, e_{z} \quad$ Projeção da excentricidade nas direções principais

$e \quad$ Altura acima do centro de gravidade à extremidade do cabo de supensão para

o içamento por pontos na face superior da viga

E Módulo de elasticidade

$\mathrm{E}_{\mathrm{a}} \quad$ Módulo de elasticidade do cabo de suspensão

$E_{1}, D_{1} \quad$ Constantes de integração

EPT Energia potencial total

$\mathrm{f}, \mathrm{g}, \mathrm{j} \quad$ Funções genéricas

$\mathrm{f}_{0} \quad$ Forças radiais distribuídas

$\mathrm{F}_{\mathrm{i}} \quad$ Funções genéricas 
FS Fator de segurança contra fissuração

FS' Fator de segurança contra ruptura

G Módulo de elasticidade transversal

h Altura da seção

$h_{\mathrm{r}} \quad$ Distância do eixo de giro à pista de rolamento

$\mathrm{i}_{\mathrm{D}} \quad$ Raio de giração polar em relação ao centro de torção

$\mathrm{i}_{z} \quad$ Raio de giração

I Momento lateral de inércia

$\mathrm{I}_{\mathrm{ef}} \quad$ Momento de inércia efetivo

$I_{y}, I_{z} \quad$ Momentos principais de inércia da seção

$\mathrm{J} \quad$ Momento de inércia das mesas

$\mathrm{J}_{1} \quad$ Momento de inércia da mesa superior

$\mathrm{J}_{2} \quad$ Momento de inércia da mesa inferior

$\mathbf{J}_{\mathrm{t}} \quad$ Momento de inércia à torção

$\mathrm{J}_{\mathrm{w}} \quad$ Momento setorial de inércia

$k, k_{1} \quad$ Parâmetros de vinculação

$\mathrm{k}_{\mathrm{c}} \quad$ Variável auxiliar

$\mathrm{K}_{\mathrm{y}}, \mathrm{K}_{\mathrm{z}}$ Coordenadas do centro do círculo de estabilidade

$\mathrm{K}_{\phi} \quad$ Constante de mola à torção

$\ell \quad$ Comprimento da viga

$\mathrm{m}, \mathrm{n}, \mathrm{t} \quad$ Variável auxiliar

M Momento fletor genérico

$\mathrm{M}_{\mathrm{c}} \quad$ Momento fletor devido à componente normal $\mathrm{F}_{0}$ na suspensão por cabos inclinados

$\mathrm{M}_{\ell} \quad$ Momento de torção livre

$\mathrm{M}_{\mathrm{ft}} \quad$ Momento de flexo-torção

$\mathrm{M}_{\mathrm{y}}, \mathrm{M}_{\mathrm{z}}$ Momentos fletores em relação aos eixos y e $\mathrm{z}$, respectivamente

$\mathrm{M}_{\mathrm{t}} \quad$ Momento total de torção

$\mathrm{N} \quad$ Força normal

$\mathrm{N}_{\mathrm{P}} \quad$ Força normal de protensão 
$\mathrm{p}, \mathrm{q} \quad$ Força uniformemente distribuída genérica

$\mathrm{p}^{*} \quad$ Carregamento genérico

$\mathrm{p}_{\mathrm{y}}, \mathrm{p}_{\mathrm{z}} \quad$ Componentes de $\mathrm{p}$ nas direções y e $\mathrm{z}$, respectivamente

$\mathrm{pp} \quad$ Carregamento relativo ao peso próprio

P Força concentrada

R Curvatura

$\mathrm{R}_{\mathrm{x}} \quad$ Constante elástica da ligação na fase de suspensão

$\mathrm{T} \quad$ Energia potencial propriamente dita

$\mathrm{T}_{2} \quad$ Energia potencial dos esforços internos de primeira ordem nas deformações de segunda ordem

$\mathrm{u}, \mathrm{v}, \mathrm{w}$ Deslocamentos genéricos segundo os eixos coordenados $\mathrm{x}, \mathrm{y}$ e $\mathrm{z}$ respectivamente

U Energia de deformação

$\mathrm{U}_{\mathrm{w}} \quad$ Característica geométrica da seção que regula a influência do bimomento na teoria de segunda ordem (Vlassov)

$v_{D}, w_{D} \quad$ Deslocamentos do centro de torção

V Volume

$\mathrm{V}_{\mathrm{y}}, \mathrm{V}_{\mathrm{z}} \quad$ Esforços cortantes nas direções y e $\mathrm{z}$, respectivamente

$\mathrm{x} \quad$ Eixo coordenado longitudinal ao elemento

$\mathrm{y}, \mathrm{z} \quad$ Eixos principais de inércia

$\mathrm{y}_{\mathrm{D}}, \mathrm{z}_{\mathrm{D}} \quad$ Coordenadas do centro de torção

$\mathrm{y}_{\mathrm{r}} \quad$ Distância do $\mathrm{CG}$ à face superior da viga

$\mathrm{z}_{\mathrm{a}}$ Distância entre a linha do CG da mesa superior e a linha do CG da linha inferior

$\mathrm{z}_{0} \quad$ Valor fictício para cálculo de $\mathrm{z}$ correspondente ao deslocamento lateral do centro de massa para todo o peso próprio aplicado lateralmente

$\mathrm{z}_{1} \quad$ Distância da face superior da viga ao eixo baricêntrico

$\mathrm{z}_{\text {máx }}$ Distância do ponto central da carroceria até o centro entre as duas rodas do eixo traseiro de 4 pneus 


\section{Gregos:}

$\alpha^{\prime}, \bar{\gamma} \quad$ Variável auxiliar

$\Delta \quad$ Flecha no meio do vão para carregamento uniformemente distribuído, e apoio nas extremidades

$\Delta_{\mathrm{y}}, \Delta_{\mathrm{z}} \quad$ Flecha no meio do vão na direção y e $\mathrm{z}$, respectivamente

$\gamma \quad$ Ângulo genérico de inclinação dos cabos de suspensão

$\delta \quad$ Deslocamento de centro de gravidade da viga

$\lambda$ Deformabilidade à torção da ligação tipo garfo

$\varepsilon^{(2)} \quad$ Deformação de segunda de ordem

$\sigma \quad$ Tensão normal

$\phi, \theta \quad$ Deslocamento angular

$\phi_{i} \quad$ Rotação inicial

$\phi_{\operatorname{máx}} \quad$ Rotação máxima relativa à fissuração

$\phi_{\text {máx }}^{\prime} \quad$ Rotação máxima relativa à ruptura

$\phi_{\mathrm{S}} \quad$ Ângulo de torção do trecho em balanço

$\tau \quad$ Tensão tangencial

$\psi \quad$ Variável auxiliar ao cálculo da carga crítica

Demais símbolos não listados, serão explicados quando de sua utilização.

Este trabalho utiliza, de forma geral, o sistema internacional de unidades (SI). Entretanto, o desenvolvimento matemático do trabalho de MAST (1993) encontra-se em outro sistema. Os fatores de conversão de unidades estão definidos a seguir:

FATORES DE CONVERSÃO DE UNIDADES (SI)

$$
\begin{array}{ll}
1 f t=0,305 \mathrm{~m} & 1 \mathrm{psi}=0,006895 \mathrm{MPa} \\
1 \text { in }=25,4 \mathrm{~mm} & 1 \mathrm{Kip}-f t=1356 \mathrm{~N}-\mathrm{m} \\
1 p c f=16,02 \mathrm{~kg} / \mathrm{m}^{3} & 1 \mathrm{Kip}=4448 \mathrm{~N} \\
1 \mathrm{lb} / f t=1,488 \mathrm{~kg} / \mathrm{m} &
\end{array}
$$




\section{RESUMO}

Este trabalho aborda o estudo da instabilidade lateral das vigas pré-moldadas durante o regime de serviço e durante a fase transitória. Na fase de serviço inclui os casos de apoios indeformáveis e deformáveis à torção. Para a fase transitória, o cálculo da carga crítica é desenvolvido para as diversas disposições dos cabos de içamento.

O estudo do equilíbrio no espaço é realizado a partir da resolução das equações diferenciais regentes da instabilidade elástica. A resolução do sistema diferencial composto pelas equações acopladas da flexão lateral e da torção é realizada por vários métodos, incluindo o desenvolvimento e automatização do método numérico de RungeKutta, para algumas seções típicas.

Apresentam-se ainda os procedimentos empíricos encontrados na literatura técnica, baseados em ensaios experimentais, para o cálculo dos fatores de segurança.

Os resultados dos exemplos numéricos mostram que as situações de serviço não são, em geral críticas, ao contrário da fase transitória. Os exemplos mostram ainda que a suspensão com balanços é uma forma de diminuir os problemas de instabilidade lateral desta fase.

Palavras-Chave: Instabilidade lateral, Vigas pré-moldadas. 


\section{ABSTRACT}

This work deals with the lateral stability of precast beams on service and during intermediate phase - handling and transportation. At service phase it is considered rigid and semi-rigid connections with torsion deformation. At handling phase, the critical load is evaluated for several positions of lifting cables.

The study of the equilibrium conditions is done with the solution of the differential equation of elastic instability problem. The solution of flexural and torsional coupled equation is gotten by use of some methods, including the one developed and authomatized from the application of Runge-Kutta method, for certain sections.

Empirical procedures are presented based on experimental tests, found in technical papers to compute fators of safety.

The results of numerical examples show that the service situations are not generally critical, unlike the intermediate situations. The results show also that a possible way to avoid this problem is with the use of lifting points with a distance from the ends.

Keywords: Lateral Instability, Precast beams. 


\section{INTRODUÇÃO}

No projeto das estruturas pré-moldadas é necessário considerar todas as etapas que compõem a produção do elemento até a sua disposição final na estrutura.

Estas fases em geral podem ser divididas em: fase de serviço, que corresponde a situação final do elemento na estrutura, e as fases transitórias.

Entende-se por fases transitórias ou temporárias, todas aquelas iniciadas na fabricação até o posicionamento definitivo na estrutura. Basicamente podem ser definidas como: fase de desmoldagem, suspensão e transporte no canteiro da fábrica, estocagem na fábrica ou no canteiro de montagem, transporte da fábrica à obra, suspensão no local da edificação e período antes de se efetuar a ligação definitiva.

Com o avanço das técnicas da protensão paralelamente ao aperfeiçoamento da tecnologia dos materiais, as seções das vigas pré-moldadas tornaram-se mais esbeltas, aumentando também os vãos empregados. Como consequência, as vigas ficaram mais susceptíveis aos problemas de instabilidade lateral. Por exemplo, as vigas que compõem a superestrutura das pontes, geralmente protendidas, de longos vãos e com seções muito altas, constituem parte dos elementos que podem apresentar excesso de deformação lateral e perda de estabilidade.

Os problemas de instabilidade não são comuns durante a fase de serviço quando 
os vínculos apresentam rigidez adequada. Em ligações deformáveis à torção, o estudo da instabilidade merece maior atenção. As fases transitórias são importantes e devem ser asseguradas quanto ao fenômeno da flambagem lateral, principalmente por apresentarem ligações incompletas e com graus de liberdade, embora o carregamento se limite ao peso-próprio. A figura 1.1 ilustra a fase de suspensão de uma viga prémoldada, por meio de cabos acoplados à face superior, exemplificando uma ligação que permite giro e deslocamentos nas seções de apoio.

De fato, problemas devido à instabilidade lateral têm ocorrido em elementos prémoldados como mostram as figuras 1.2 e 1.3. O colapso de uma viga de ponte, de seção alta e grande comprimento, ilustrado na figura 1.2, corresponde a uma situação de perda de estabilidade durante a fase de tranporte em rodovia. A figura 1.3 apresenta uma viga de cobertura de galpões em estado de ruína por flambagem lateral.

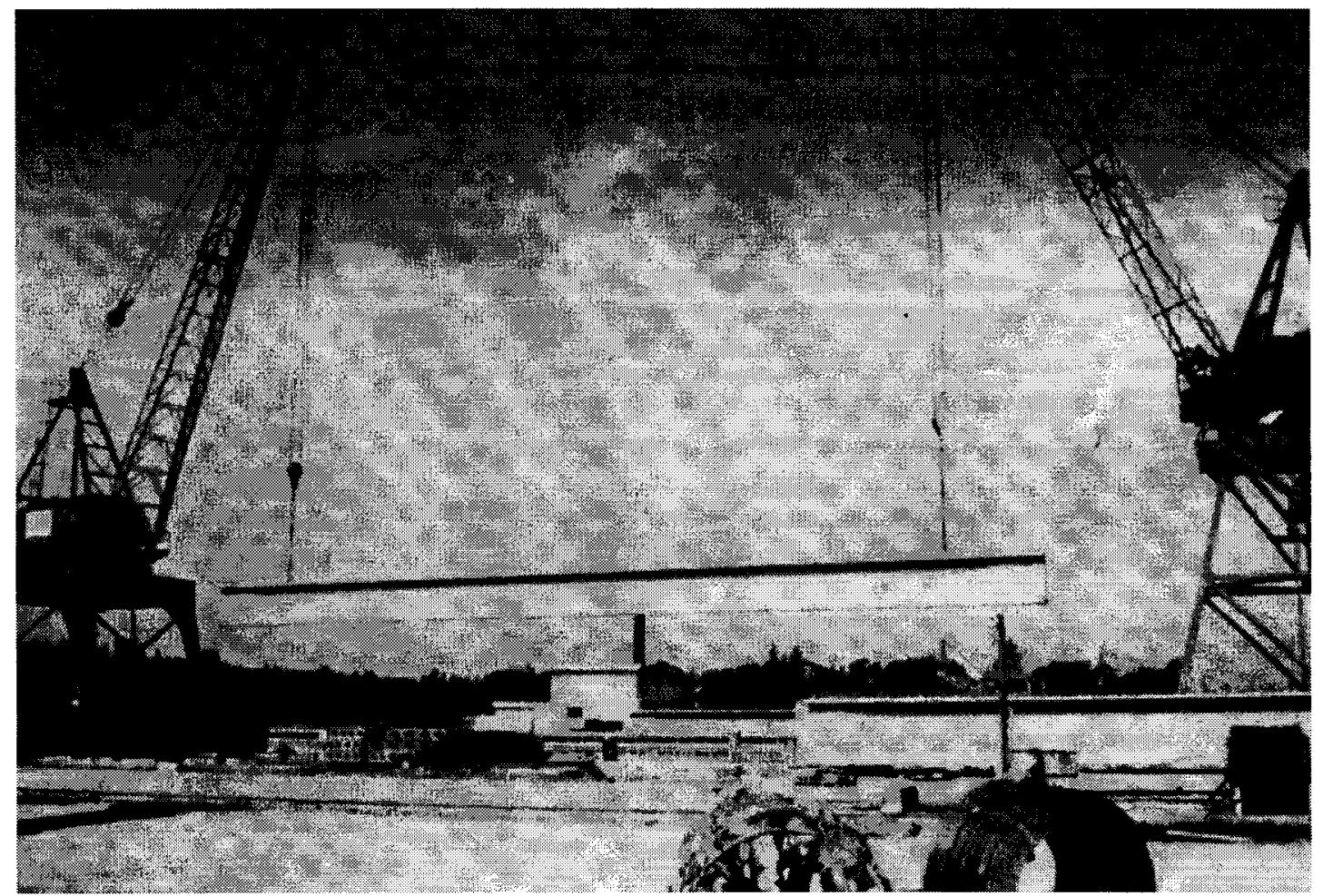

Figura 1.1 - Manuseio de uma viga pré-moldada de concreto. [ IMPER \& LASZLO (1987)]

A estabilidade das vigas depende não apenas da capacidade das mesmas de resistir aos esforços solicitantes convencionais, como o peso-próprio e a protensão; mas 
sobretudo, resistir às deformações laterais significativas que ocorrem nesta fase, em razão da esbeltez da seção. Para isso, os elementos pré-moldados devem ter rigidez lateral suficiente para evitar a redução da capacidade resistente, por excesso de deformação e fissuração.

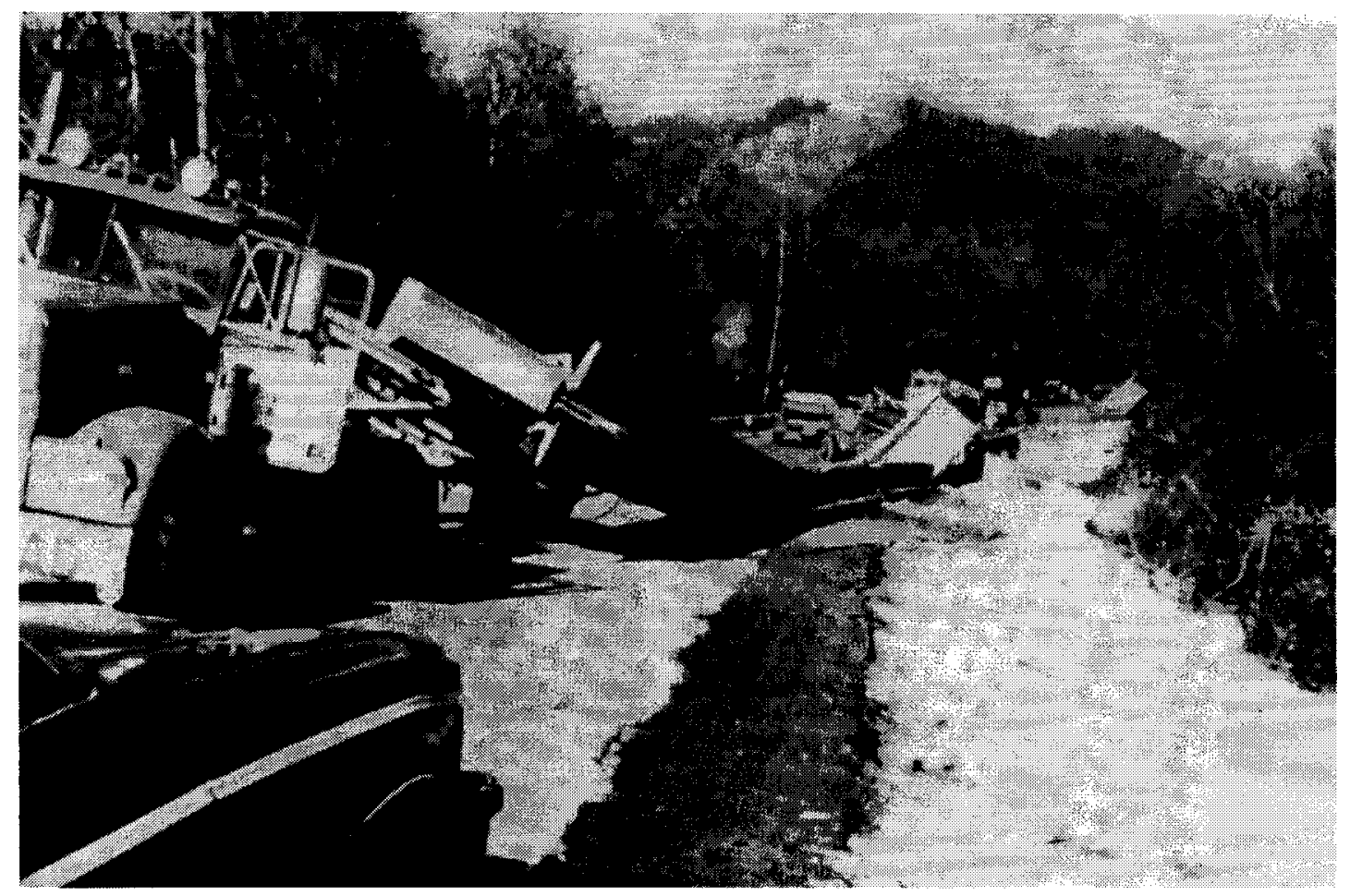

Figura 1.2 - Colapso de uma viga pré-moldada de concreto durante o transporte. [ IMPER \& LASZLO (1987) ]

Como referido anteriormente, a maioria dos problemas de instabilidade lateral em vigas longas e delgadas ocorrem quando os apoios apresentam liberdade ao giro por flexão e torção. Estes vínculos podem ser os cabos de suspensão, que são ligações provisórias, ou também, as ligações deformáveis da estrutura. Em adição aos graus de liberdade fornecidos por estes vínculos, temporários ou definitivos da estrutura, a verificação da estabilidade dos elementos torna-se mais crítica quando são consideradas as inevitáveis imperfeições construtivas e os desvios de montagem.

As imperfeições geradas na fase construtiva do elemento pré-moldado são basicamente provocadas pelo controle ineficaz do posicionamento das armaduras, pela não homogeneidade da peça moldada, pelos erros de posicionamento das fontes de calor 
na cura acelerada, pela má execução da protensão, apresentando perdas excessivas e não controladas.

O posicionamento dos pontos de suspensão feito de forma imprevista, por exemplo o içamento com balanços, pode acarretar uma série de inconvenientes ao elemento, resultando no aparecimento de tensões de tração e possível fissuração de algumas seções, as quais não foram dimensionadas para estes esforços solicitantes.

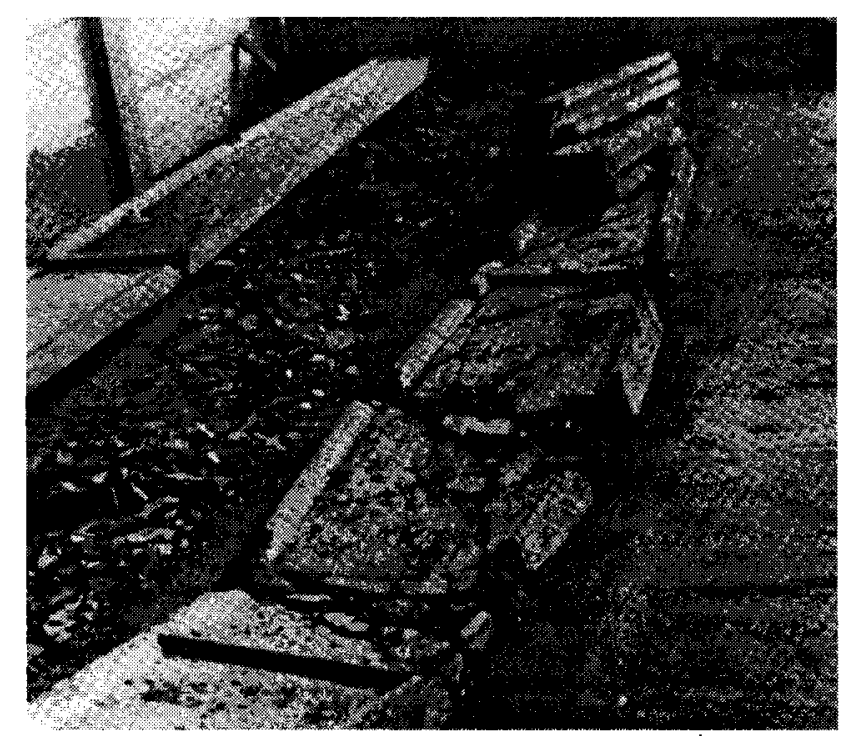

Figura 1.3 - Colapso de uma viga por instabilidade lateral. [ CATANIA \& COCCHI (1976) ]

Durante o transporte das vigas longas, os efeitos dinâmicos variam em intensidade conforme as condições da pista de rolamento, a elasticidade do sitema de amortecimento do meio e dos vínculos, a elasticidade do elemento estrutural, dentre outros fatores. $\mathrm{O}$ aparecimento das fissuras nas seções das vigas pode ser provocado pelas excessivas solicitações causadas por um incorreto ou mal posicionamento do elemento na carreta. Também ocorrem durante a passagem do veículo sobre as curvas e as superelevações, cujos desníveis, não sendo compensados pelo sistema hidráulico do aparelho de apoio, resultam em esforços de torção ao longo do comprimento da viga. Estes fatores contribuem de forma singular com a instabilidade do elemento.

A norma brasileira NBR-9062 (1985), Projeto e execução de estruturas de concreto pré-moldado, no item 6.1 relativo à estabilidade lateral das vigas pré-moldadas, 
limita-se aos princípios básicos e poucas indicações gerais de projeto, o que justifica a necessidade de um estudo mais detalhado da possibilidade de perda de estabilidade das vigas esbeltas.

O texto base para revisão da NB-1/78 (1994), sobre a estabilidade lateral de vigas de concreto armado, apesar de não ser específico para as vigas pré-moldadas, considera importante assegurar a estabilidade lateral das vigas de seção delgada na fase de serviço através de limitações da curvatura, sem ainda quantificar este limite.

\subsection{REVISÃO BIBLIOGRÁFICA}

As vigas, mesmo carregadas apenas no plano vertical, podem romper-se por deslocamentos laterais excessivos e rotações por torção, simultaneamente. Este tipo de colapso é chamado de flambagem lateral e foi analisado primeiramente por Prandtl, em 1899. Em 1940, a teoria proposta por Vlassov para barras de seção delgada consiste em importante avanço no estudo da instabilidade de peças estruturais unidimensionais.

Os fundamentos da instabilidade lateral de vigas baseados na teoria de Vlassov constituem no estudo em regime elástico do equilíbrio em teoria de $2^{\mathrm{a}}$ ordem, considerando o equilíbrio geral no espaço de vigas esbeltas de esqueleto aberto.

As expressões diferenciais regentes da instabilidade lateral em vigas de seção delgada, encontram-se definidas em RACHID (1993) pelo método da energia, no estudo do equilíbrio do elemento na posição deslocada.

O estudo da instabilidade lateral de vigas pré-moldadas apresenta-se na literatura de forma restrita, com poucos trabalhos desenvolvidos neste assunto.

CATANIA \& COCCHI (1985), para algumas seções típicas de concreto, tratam dos problemas da instabilidade das vigas pré-moldadas, em regime de serviço e durante a fase transitória. Em razão da frequente utilização de seções de vigas baseadas nas formas usuais dos perfis metálicos, os autores ressaltam a importância de prevenir o fenômeno da instabilidade por flexão lateral e torção.

IMPER \& LASZLO (1987) no estudo da instabilidade durante o manuseio de 
longas vigas de pontes, sugerem um procedimento analítico e empírico para determinação da segurança durante a fase transitória. Especial atenção foi dispensada à influência do posicionamento dos pontos de apoio, nas duas etapas consideradas da fase transitória, à benefício da estabilidade da viga. Os sistemas de contraventamento usuais são discutidos no trabalho citado.

O estudo desenvolvido por MAST (1989) avalia a estabilidade lateral de vigas protendidas de seção duplo $\mathrm{T}$, quando suspensas por cabos de elevação, através da definição de um fator de segurança. Este fator depende da altura do eixo de giro (este eixo é a reta que une o centro de giro de cada apoio e, normalmente situa-se na superfície superior da viga de concreto), da excentricidade lateral inicial, da rigidez lateral e da máxima inclinação permissível da viga.

Na continuação da pesquisa, MAST (1993) extende as análises de estabilidade lateral para casos mais gerais de vigas, cujo suporte seja provido de restrições elásticas ao giro. Inclui vigas apoiadas em almofadas de elastômero e em caminhões, durante o transporte em via de tráfego. A superelevação da via é admitida e alguns procedimentos simples são descritos para sua determinação. Para a fase de suspensão, os fatores de segurança alcançaram maior amplitude, e o estudo realizado anteriormente pelo autor foi reformulado pela avaliação da fissuração e do colapso da viga, com comprovação experimental por ensaios em escala real.

O estudo de CUNHA (1994), relativo aos problemas de estabilidade de vigas esbeltas protendidas durante o içamento e o transporte, foi desenvolvido através da análise de diferentes parâmetros referentes à estabilidade. Apresenta uma série de gráficos de influência, baseados em MAST (1989, 1993). Entretanto, os gráficos pertecem a um caso específico, e de forma geral, servem para mostrar qualitativamente a importância dos parâmetros na estabilidade de vigas protendidas esbeltas e de grande comprimento.

Os parâmetros avaliados para a fase de suspensão foram: a força de protensão, a posição da resultante de protensão, a posição dos pontos de içamento e a resistência do concreto. Para a fase de transporte da fábrica ao canteiro de obras: locação dos apoios da viga sobre o veículo, força de protensão, posição da resultante dos cabos de 
protensão, distância do CG à pista de rolamento, superelevação da estrada e rigidez da carroceria.

Uma contribuição dada por CUNHA (1994), e que será descrita neste trabalho, refere-se à determinação experimental do coeficiente de rigidez à rotação da carroceria do veículo utilizado para o transporte das vigas, bem como a medida da superelevação da via de tráfego por onde o veículo que transporta as vigas deve passar. Estes dados são necessários à verificação da estabilidade das vigas durante o transporte.

\subsection{OBJETIVOS}

Tendo em vista a importância da verificação da estabilidade de vigas prémoldadas em todas as suas fases de projeto, os objetivos deste trabalho são:

a.) o estudo da instabilidade lateral de vigas pré-moldadas durante a fase em serviço, incluindo a consideração da deformabilidade à torção dos apoios;

b.) o estudo da instabilidade lateral de vigas pré-moldadas durante a fase transitória de suspensão, para várias disposições dos cabos de içamento;

c.) comparar os resultados do estudo da instabilidade lateral nas situações transitórias com os modelos simplificados encontrados na literatura.

\subsection{APRESENTAÇÃO DO TRABALHO}

No capítulo 2 encontra-se desenvolvido o estudo da instabilidade lateral das vigas durante a fase de serviço. Esta fase será analisada para vínculos rígidos e deformáveis à torção.

As vigas protendidas também serão avaliadas quanto à instabilidade, considerando isoladamente os efeitos da protensão, e posteriormente, em ação conjunta com o carregamento aplicado. 
A fase transitória de suspensão está descrita no capítulo 3, para as diversas disposições dos cabos: cabos oblíquos, inclinados e retos. $\mathrm{O}$ número de pontos de içamento variam, bem como a sua locação na face superior da viga.

Foram empregados neste capítulo, o método de integração por séries de potência, o método de Ritz, e o método numérico de Runge-Kutta, especialmente programado para o estudo da instabilidade de vigas em suspensão.

Para as vigas protendidas, o estudo da flambagem lateral no içamento por cabos inclinados será apresentado para carregamentos distribuídos, representados de forma genérica através das séries de Fourier.

No capítulo 4 apresentam-se os procedimentos empíricos para a determinação dos fatores de segurança relativos à fase transitória de suspensão e de transporte, considerando a fissuração e a ruptura, baseado no trabalho de $\operatorname{MAST}(1989,1993)$.

Os exemplos numéricos e as análises dos resultados compõem o capítulo 5, onde serão analisados quanto aos problemas de instabilidade lateral, em serviço e durante a suspensão, uma viga de seção retangular de concreto armado, uma viga de argamassa armada de seção duplo T simétrico e uma viga protendida de seção duplo T do PCI.

O capítulo 6 trata das considerações finais e conclusões. 


\section{INSTABILIDADE LATERAL DE VIGAS DURANTE A FASE DE SERVIÇO}

\subsection{PRELIMINARES}

O estudo da estabilidade lateral das vigas pré-moldadas, como as vigas de argamassa armada, com longos vãos, tem merecido maior atenção devido à utilização de elementos cada vez mais delgados. Este estudo torna-se ainda mais importante em regime de serviço, tanto nas vigas de concreto como nas vigas de argamassa armada, à medida que se admitem ligações deformáveis à torção.

As vigas de concreto por não apresentarem geralmente seções delgadas, mas com grande rigidez à torção, dificilmente submetem-se a fenômenos de flambagem lateral. Entretanto, a causa da maioria dos problemas de perda de estabilidade em vigas longas de concreto é a deformabilidade dos apoios.

No estudo do equilíbrio geral no espaço em teoria de segunda ordem, será avaliado através da carga crítica, o valor do carregamento que provoca mudança no tipo de equilíbrio em regime elástico.

A determinação da carga crítica no estudo do equilíbrio na posição deformada, resulta do desenvolvimento das equações diferenciais. A resolução destas equações 
geralmente é inviável pelo método exato, devido à complexidade de manuseio das funções de Bessel. As soluções por métodos numéricos e aproximados são, na maioria das vezes preferidas, apresentando excelentes resultados.

As equações diferenciais regentes da instabilidade lateral encontram-se desenvolvidas em RACHID (1993), pelo método da energia potencial. O procedimento de obtenção destas equações parte do estudo do equilíbrio na posição deslocada considerada suficientemente próxima da posição original, de modo que possam ser adotadas as expressões aproximadas para os deslocamentos e deformações.

As expressões diferenciais gerais da instabilidade lateral definidas em RACHID (1993), segundo a teoria de Vlassov, para carregamentos como: força transversal distribuída, força transversal concentrada de componentes nos eixos principais (com a restrição de passarem pelo centro de torção), momentos fletores e bimomentos aplicados, são:

$$
\begin{aligned}
& E I_{y} v_{D}^{\prime \prime \prime \prime}-\left[N\left(v_{D}^{\prime}+z_{D} \phi^{\prime}\right)\right]^{\prime}-\left(M_{y} \phi\right)^{\prime \prime}-p_{y}=0 \\
& E I_{y} w_{D}^{\prime \prime \prime \prime}-\left[N\left(w_{D}^{\prime}+y_{D} \phi^{\prime}\right)\right]^{\prime}+\left(M_{z} \phi\right)^{\prime \prime}-p_{z}=0 \\
& E J_{w} \phi^{\prime \prime \prime \prime}-G J_{t} \phi^{\prime \prime}-\left\{\left[N i_{D}^{2}+2 M_{z}\left(K_{y}-y_{D}\right)+\right.\right. \\
& \left.\left.\quad+2 M_{y}\left(K_{z}-z_{D}\right)+\frac{B}{J_{w}} U_{w}\right] \phi^{\prime}\right\}^{\prime}-\left(N w_{D}^{\prime}\right)^{\prime} y_{D}+ \\
& \quad+\left(N v_{D}^{\prime}\right)^{\prime} z_{D}+M_{z} w_{D}^{\prime \prime}-M_{y} v_{D}^{\prime \prime}-\left(p_{y} e_{y}+p_{z} e_{z}\right) \phi=0
\end{aligned}
$$

Para a resolução das equações diferenciais regentes da instabilidade lateral CATANIA \& COCCHI (1985) utilizaram o método de integração por séries de potência e o método de Ritz. O método numérico de Runge-Kutta foi escolhido neste trabalho para ser programado com o fim de determinar a carga crítica, a partir do sistema de equações diferenciais regentes da instabilidade e das condições de contorno.

O método de Runge-Kutta é eficaz e de grande importância prática porque não necessita de procedimentos especiais e utiliza o mesmo procedimento várias vezes. Dentre as formulações por passos, é amplamente utilizado por gerar resultados precisos. Uma vantagem que apresenta na resolução de um sistema de equações diferenciais é a simplicidade da programação em problemas de valor inicial, valor de contorno e auto- 
valor, onde as condições de contorno são incorporadas.

Uma comparação entre os resultados da carga crítica obtidos com o método de integração por séries de potência e o método de Runge-Kutta, aplicados à equação diferencial de uma viga de seção retangular sobre vínculos de garfo indeformável, indica valores totalmente satisfatórios.

Este capítulo basicamente está subdividido em duas partes. A primeira refere-se ao estudo da instabilidade lateral das vigas em serviço sobre apoios indeformáveis e a segunda, sobre apoios deformáveis à torção.

\subsection{EQUAÇÕES DIFERENCIAIS REGENTES DA INSTABILIDADE LATERAL PARA ALGUMAS SEÇÕES TÍPICAS}

A análise do equilíbrio geral de vigas no espaço é expressa, para cada tipo de seção e carregamento, por um sistema de equações diferenciais que incluem a flexão em relação aos eixos principais de inércia e a torção. No estudo da instabilidade, geralmente estão acopladas as equações representativas da flexão lateral e da torção.

O trabalho de CATANIA \& COCCHI (1985) apresenta uma dedução particular para três seções comuns de elementos pré-moldados de concreto pelo método do equilíbrio. No estudo de RACHID (1993), as equações gerais da instabilidade deduzidas pelo método da energia potencial são a base do processo de Ritz, as quais podem ser aplicadas a qualquer tipo de seção transversal. Estas equações consideram os mesmos tipos de carregamento citados para a equação (2.1).

Nos quadros 2.1 e 2.2 encontram-se as equações diferenciais definidas para algumas seções. Estas seções transversais são constantes ao longo do comprimento e das formas: retangular, duplo T simétrico e duplo T não simétrico. O carregamento é linear distribuído na linha do CG e a viga simplesmente apoiada nas extremidades em vínculos de garfo. As expressões diferenciais regentes da instabilidade lateral definidas nos quadros, para cada tipo de seção, são equivalentes. 
Quadro 2.1 - Equações diferenciais regentes da instabilidade lateral. [ RACHID (1993) ]

\begin{tabular}{|c|c|}
\hline Seção & Equações diferenciaịs \\
\hline retangular & $\phi^{\prime \prime}(x)+\frac{M_{z}^{2}}{E I_{y} G J_{t}} \phi(x)=0$ \\
\hline $\begin{array}{l}\text { duplo } \mathrm{T} \\
\text { simétrico }\end{array}$ & $\phi^{\prime \prime \prime \prime}(x)-\frac{G J_{t}}{E J_{w}} \phi^{\prime \prime}(x)-\frac{M_{z}^{2}}{E I_{y} E J_{w}} \phi(x)=0$ \\
\hline $\begin{array}{c}\text { duplo } \mathrm{T} \\
\text { não simétrico }\end{array}$ & $\phi^{\prime \prime \prime \prime}(x)-\frac{G J_{t}}{E J_{w}} \phi^{\prime \prime}(x)-\frac{M_{z}^{2}}{E I_{y} E J_{w}} \phi(x)=0$ \\
\hline
\end{tabular}

Quadro 2.2 - Equações diferenciais regentes da instabilidade lateral. [ CATANIA \& COCCHI (1985) ]

\begin{tabular}{|c|c||}
\hline Seção & Equações diferenciais \\
\hline retangular & $\phi^{\prime \prime}(x)+\frac{M_{y}^{2}}{E I_{z} G J_{t}} \phi(x)=0$ \\
\hline $\begin{array}{c}\text { duplo T } \\
\text { simétrico }\end{array}$ & $-\frac{1}{2} E J z_{a}^{2} \phi^{\prime \prime \prime}(x)+G J_{t} \phi^{\prime \prime}(x)+\frac{M_{y}^{2}(x)}{E I_{z}} \phi(x)=0$ \\
\hline duplo T & $\cdot \cdot M_{y}^{2}(x)$ \\
não simétrico & $-E \frac{J_{1} J_{2}}{J_{1}+J_{2}} z_{a}^{2} \phi^{\prime \prime \prime \prime}(x)+G J_{t} \phi^{\prime \prime}(x)+\frac{0}{2}$ \\
\hline
\end{tabular}

A nomenclatura utilizada no quadro 2.2 pode ser traduzida por:

J - momento de inércia das mesas (no caso de mesas simétricas);

$\mathrm{J}_{1}$ - momento de inércia da mesa superior;

$\mathrm{J}_{2}$ - momento de inércia da mesa inferior; 
$\mathrm{Z}_{\mathrm{a}}$ - distância entre a linha do CG da mesa superior e a linha do CG da mesa inferior.

Os eixos coordenados definidos por CATANIA \& COCCHI (1985) são contrários aos apresentados em RACHID (1993), cuja convenção de sinais dos eixos será adotada neste trabalho.

\subsection{RESOLUÇÃO DA EQUAÇÃO DIFERENCIAL PARA VIGAS RETANGULARES}

O cálculo da expressão da carga crítica para uma viga de seção retangular apoiada sobre garfos nas extremidades será desenvolvido por dois métodos numéricos: séries de potência, conforme CATANIA \& COCCHI (1985) e Runge-Kutta, processo automatizado computacionalmente neste trabalho. $\mathrm{O}$ carregamento $\mathrm{p}$ é uniformemente distribuído na linha do eixo baricêntrico.

O conceito dos vínculos de garfo pode ser entendido como o apoio que é capaz de impedir a rotação por torção deixando livre os deslocamentos axiais, os quais são chamados de empenamento. A deformabilidade dos garfos à torção não será ainda considerada.

\subsubsection{PROCESSO DE INTEGRAÇÃO POR SÉRIES DE POTÊNCIA}

A viga de seção retangular ilustrada na figura 2.1 será avaliada quanto ao valor crítico de carregamento linear unifome $\mathrm{p}$ que provoca perda de estabilidade lateral.

A expressão do momento fletor no plano vertical $M=M_{z}$ é:

$$
M(x)=M(m)=\frac{1}{2} p a^{2}\left(1-m^{2}\right) .
$$

onde $\mathrm{m}$ é uma variável auxiliar definida por $\mathrm{m}=2 \mathrm{x} / \ell$ no intervalo $(-1,1)$. 


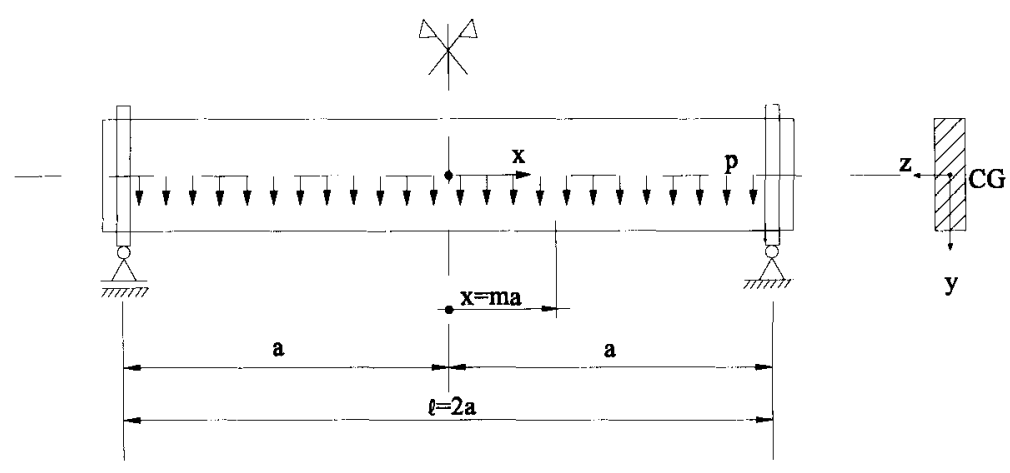

Figura 2.1 - Nomenclatura e sistema de eixos coordenados segundo CATANIA \& COCCHI (1985)

A equação diferencial regente da instabilidade lateral está definida a seguir:

$$
\phi^{\prime \prime}(x)+\frac{M^{2}(x)}{E I_{y} G J_{t}} \phi(x)=0
$$

Relacionando a derivada segunda de $\phi(\mathrm{x})$ com a derivada segunda de $\phi(\mathrm{m})$, temse para $\mathrm{dm} / \mathrm{dx}=2 / \ell$ e $\ell=2 \mathrm{a}$ :

$$
\begin{gathered}
\frac{d \phi}{d x}=\frac{d \phi}{d m} \frac{d m}{d x} \\
\frac{d^{2} \phi(x)}{d x^{2}}=\frac{1}{a^{2}} \cdot \frac{d^{2} \phi(m)}{d m^{2}}
\end{gathered}
$$

Substituindo a equação (2.4) na expressão diferencial (2.3) resulta:

$$
\phi^{\prime \prime}(m)+\frac{1}{4} \frac{p^{2} a^{6}}{E I_{y} G J_{t}}\left(1-m^{2}\right)^{2} \phi(m)=0
$$

Definindo $\alpha$ como:

$$
\alpha=\frac{p^{2} a^{6}}{4 E I_{y} G J_{t}}
$$

tem-se:

$$
\phi^{\prime \prime}(m)+\alpha\left(1-m^{2}\right)^{2} \phi(m)=0
$$

A resolução da equação acima em forma de série inteira pode ser expressa por: 


$$
\begin{gathered}
\phi(m)=\phi_{0}(m)+\phi_{1}(m)=\sum_{i-2 j}^{\infty} A_{i} m^{i}+\sum_{k-2 j+1}^{\infty} A_{k} m^{k} \\
(j=0,1,2, \ldots)
\end{gathered}
$$

onde $\phi_{0}(\mathrm{~m})$ representa os termos com índices pares e $\phi_{1}(\mathrm{~m})$ representa os termos com índices ímpares da série.

$\mathrm{O}$ número de elementos da série a ser considerado depende do grau de aproximação que se deseja obter. CATANIA \& COCCHI (1985) optaram por adotar um polinômio com 10 coeficientes desconhecidos: de $A_{0}$ à $A_{9}$. Os coeficientes $A_{10}$ e $A_{12}$ foram introduzidos nas derivadas para melhorar a precisão fornecida pelos polinômios.

Por simetria, a equação de $\phi$ deve resultar em uma função par de m, então:
(a) $\phi(m)=\phi_{0}(m)=\sum_{i=2 j}^{\infty} A_{i} m^{i}$
(b) $\phi_{0}(m)=A_{0} m^{0}+A_{2} m^{2}+A_{4} m^{4}+A_{6} m^{6}+A_{8} m^{8}$
(c) $\phi_{0}^{\prime}(m)=2 A_{2} m+4 A_{4} m^{3}+6 A_{6} m^{5}+8 A_{8} m^{7}+10 A_{10} m^{9}$
(d) $\phi^{\prime \prime}{ }_{0}(m)=2 A_{2}+12 A_{4} m^{2}+30 A_{6} m^{4}+56 A_{8} m^{6}+90 A_{10} m^{8}+132 A_{12} m^{10}$

Substituindo os polinômios de $\phi(\mathrm{m})$ e $\phi^{\prime \prime}(\mathrm{m})$ na equação diferencial, tem-se:

$$
\begin{aligned}
{\left[2 A_{2}\right.} & \left.+12 A_{4} m^{2}+30 A_{6} m^{4}+56 A_{8} m^{6}+90 A_{10} m^{8}+132 A_{12} m^{10}\right]+ \\
& +\alpha\left(1-m^{2}\right)^{2}\left[A_{0}+A_{2} m^{2}+A_{4} m^{4}+A_{6} m^{6}+A_{8} m^{8}\right]=0
\end{aligned}
$$

e agrupando os termos na variável m:

$$
\begin{aligned}
& \quad\left(2 A_{2}+\alpha A_{0}\right)+ \\
& +\left(12 A_{4}+\alpha A_{2}-2 \alpha A_{0}\right) m^{2}+ \\
& +\left(30 A_{6}+\alpha A_{4}-2 \alpha A_{2}+\alpha A_{0}\right) m^{4}+ \\
& +\left(56 A_{8}+\alpha A_{6}-2 \alpha A_{4}+\alpha A_{2}\right) m^{6}+ \\
& +\left(90 A_{10}+\alpha A_{8}-2 \alpha A_{6}+\alpha A_{4}\right) m^{8}+ \\
& +\left(132 A_{12}-2 \alpha A_{8}+\alpha A_{6}\right) m^{10}+\left(\alpha A_{8}\right) m^{12}=0
\end{aligned}
$$


Então, ou $\mathrm{m}=0$ que resulta na solução trivial, ou os coeficientes da expressão (2.11) devem ser nulos. Assim:

$$
\begin{aligned}
& \alpha A_{0}+2 A_{2}=0 \\
& \alpha\left(-2 A_{0}+A_{2}\right)+12 A_{4}=0 \\
& \alpha\left(A_{0}-2 A_{2}+A_{4}\right)+30 A_{6}=0 \\
& \alpha\left(A_{2}-2 A_{4}+A_{6}\right)+56 A_{8}=0 \\
& \alpha\left(A_{4}-2 A_{6}+A_{8}\right)+90 A_{10}=0 \\
& \alpha\left(A_{6}-2 A_{8}\right)+132 A_{12}=0 \\
& \alpha\left(A_{8}\right)=0
\end{aligned}
$$

de forma que as expressões dos coeficientes são:

$$
\begin{aligned}
& A_{2}=-\frac{1}{2} \alpha A_{0} \\
& A_{4}=\left(\frac{\alpha}{6}+\frac{\alpha^{2}}{24}\right) A_{0} \\
& A_{6}=\left(-\frac{\alpha}{30}-\frac{7 \alpha^{2}}{180}-\frac{\alpha^{3}}{720}\right) A_{0} \\
& A_{8}=\left(\frac{13 \alpha^{2}}{840}+\frac{11 \alpha^{3}}{5040}+\frac{\alpha^{4}}{40320}\right) A_{0}
\end{aligned}
$$

Substituindo as equações obtidas em (2.13) na expressão (2.9b):

$$
\begin{aligned}
\phi_{0}(m)= & A_{0}\left[1-\frac{1}{2} \alpha m^{2}+\left(\frac{\alpha}{6}+\frac{\alpha^{2}}{24}\right) m^{4}+\right. \\
& +\left(-\frac{\alpha}{30}-\frac{7 \alpha^{2}}{180}-\frac{\alpha^{3}}{720}\right) m^{6}+ \\
& \left.+\left(\frac{13 \alpha^{2}}{840}+\frac{11 \alpha^{3}}{5040}+\frac{\alpha^{4}}{40320}\right) m^{8}\right]
\end{aligned}
$$

Aplicando as condições de contorno, onde o vínculo de garfo rígido fornece giro nulo por torção, tem-se: 


$$
\phi(m) \underset{m= \pm 1}{\mid}=0
$$

Desprezando os termos com potência $\alpha$ maior que $2 \mathrm{e}$, acrescentando os termos devido às parcelas de $A_{10}$ e $A_{12}$, obtém-se a igualdade que segue:

$$
\begin{gathered}
1-\frac{1}{2} \alpha+\frac{\alpha}{6}+\frac{\alpha^{2}}{24}-\frac{\alpha}{30}+\frac{13 \alpha^{2}}{840}-\frac{7 \alpha^{2}}{2700}+\frac{\alpha^{2}}{3960}=0 \\
\frac{6617}{415800} \alpha^{2}-\frac{11}{30} \alpha+1=0 \\
\therefore \quad \alpha_{\text {crtt }}=3,16
\end{gathered}
$$

O valor crítico de $\alpha$ substituído na expressão (2.6) fornece o carregamento crítico:

$$
\alpha_{c r t t}=\frac{p_{c r t t}^{2} a^{6}}{4 E I_{z} G J}
$$

Isolando $p_{\text {crit }}$ na equação acima:

$$
p_{\text {crtt }}=28,44 \frac{\sqrt{E I_{y} G J_{t}}}{\ell^{3}}
$$

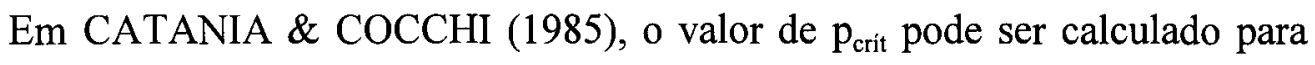
diversas condições de vinculação, procedendo analogamente ao desenvolvido, mas fazendo as devidas modificações nas expressões diferenciais relativas à flexão e à torção.

Generalizando, a expressão da carga crítica para os tipos de vinculações definidos na tabela 2.1 é:

$$
p_{c r i t}=k \frac{\sqrt{E I_{y} G J_{t}}}{\ell^{3}}
$$

\subsubsection{MÉTODO DE RUNGE-KUTTA}

A viga da figura 2.2, para o sistema adotado de eixos coordenados, será agora 
avaliada quanto à instabilidade lateral utilizando o método numérico de Runge-Kutta na solução da equação diferencial regente.

Tabela 2.1 - Valores de $k$ relativos ao tipo de apoio. [ CATANIA \& COCCHI (1985) ]

\begin{tabular}{|c|c|c|c|}
\hline \multicolumn{4}{|c|}{ VÍNCULOS NAS SEÇÕES DE EXTREMIDADE } \\
\hline À torção & À flexão vertical & À flexão lateral & $\mathrm{k}$ \\
\hline \multirow{6}{*}{$\begin{array}{l}\text { Engaste } \\
\text { perfeito }\end{array}$} & $\begin{array}{l}\text { apoio simples / } \\
\text { apoio simples }\end{array}$ & $\begin{array}{l}\text { garfo / } \\
\text { garfo }\end{array}$ & 28,4 \\
\hline & $\begin{array}{l}\text { engaste / } \\
\text { extremidade livre }\end{array}$ & $\begin{array}{l}\text { garfo / } \\
\text { extremidade livre }\end{array}$ & 12,85 \\
\hline & $\begin{array}{l}\text { engaste / } \\
\text { engaste }\end{array}$ & $\begin{array}{l}\text { garfo / } \\
\text { garfo }\end{array}$ & 98,0 \\
\hline & $\begin{array}{l}\text { engaste / } \\
\text { apoio simples }\end{array}$ & $\begin{array}{l}\text { garfo / } \\
\text { garfo }\end{array}$ & 54,0 \\
\hline & $\begin{array}{l}\text { apoio simples / } \\
\text { apoio simples }\end{array}$ & $\begin{array}{l}\text { engaste perfeito / } \\
\text { engaste perfeito }\end{array}$ & 50,0 \\
\hline & $\begin{array}{l}\text { engaste perfeito / } \\
\text { engaste perfeito }\end{array}$ & $\begin{array}{l}\text { engaste perfeito / } \\
\text { engaste perfeito }\end{array}$ & 137,0 \\
\hline
\end{tabular}

A seção é duplamente simétrica, o centro de torção coincide com o CG e, portanto, as coordenadas do centro de torção $\mathrm{y}_{\mathrm{D}}$ e $\mathrm{z}_{\mathrm{D}}$ são nulas. A seção retangular não apresenta características setoriais, de forma que o momento sẹtorial de inércia $\mathrm{J}_{\mathrm{w}}$ é igual a zero.

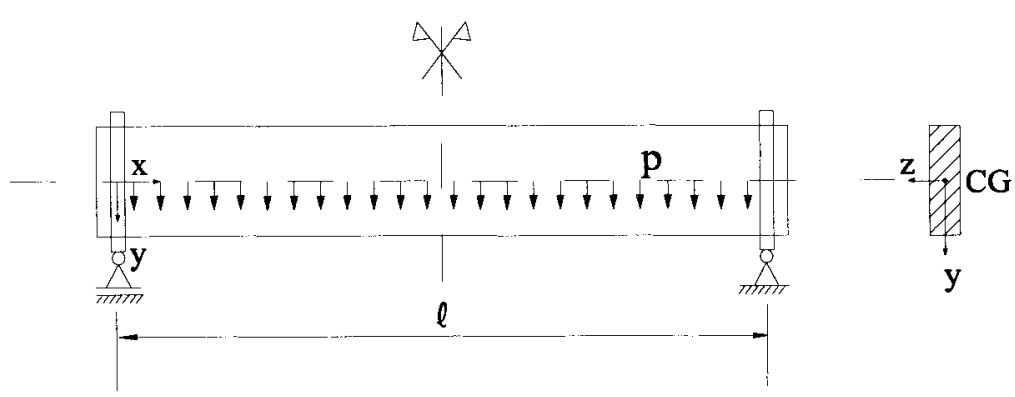

Figura 2.2 - Viga de seção retangular apoiada sobre garfos. 
As equações diferenciais regentes são:

$$
\begin{aligned}
& \text { (a) } E I_{z} v_{D}^{\prime \prime \prime \prime}-p=0 \\
& \text { (b) } E I_{y} w_{D}^{\prime \prime \prime \prime}+\left(M_{z} \phi\right)^{\prime \prime}=0 \\
& \text { (c) }-G J_{t}^{\prime \prime \prime} \phi^{\prime \prime} M_{z} w_{D}^{\prime \prime}=0
\end{aligned}
$$

A equação (2.21a) resulta na expressão já muito conhecida da resistência dos materiais, correspondente à flexão no plano vertical em $1^{\mathrm{a}}$ ordem:

$$
v_{D}(x)=\frac{p}{E I_{z}}\left(\frac{x^{4}}{24}-\frac{\ell x^{3}}{12}+\frac{\ell^{3} x}{24}\right)
$$

Para $x=\ell / 2$, a flecha $v_{D}$ vale:

$$
\operatorname{mó} x v_{D}(\ell / 2)=\frac{5 p \ell^{4}}{384 E I_{z}}
$$

As equações (2.21b) e (2.21c) acopladas serão resolvidas a seguir. Fazendo a integração preliminar da equação (2.21b), tem-se:

$$
\begin{gathered}
E I_{y} w_{D}^{\prime \prime \prime}+\left(M_{z} \phi\right)^{\prime}=D_{1} \\
E I_{y} w_{D}^{\prime \prime}+M_{z} \phi=D_{1} x+E_{1}
\end{gathered}
$$

onde $D_{1}$ e $E_{1}$ são constantes de integração genéricas.

Aplicando as condições de contorno em $\mathrm{w}_{\mathrm{D}}$, obtém-se $\mathrm{E}_{1}=\mathrm{D}_{1}=0$.

$$
\begin{array}{rllll}
w_{D}^{\prime \prime}(0)=0 & e & \phi(0)=0 & \Rightarrow & E_{1}=0 \\
w_{D}^{\prime \prime \prime}(0)=0 & e & M_{z}(0)=0 & \Rightarrow & D_{1}=0
\end{array}
$$

As expressões dos esforços de $2^{\mathrm{a}}$ ordem são:

$$
\begin{aligned}
& M_{y}\left(2^{\mathrm{a}}\right)=-E I_{y} w_{D}^{\prime \prime} \\
& V_{z}\left(2^{\mathrm{a}}\right)=-E I_{y} w_{D}^{\prime \prime \prime}
\end{aligned}
$$

de forma que, a expressão diferencial da elástica em $\mathrm{w}_{\mathrm{D}}$ é: 


$$
E I_{y} w_{D}^{\prime \prime}+M_{z} \phi=0
$$

Logo,

$$
w_{D}^{\prime \prime}=-\frac{M_{z}}{E I_{y}} \phi
$$

Substituindo $\mathrm{w}_{\mathrm{D}}$ " na equação $(2.21 \mathrm{c})$, resulta:

$$
G J_{t} \phi^{\prime \prime}(x)+\frac{M_{z}^{2}}{E I_{y}} \phi(x)=0
$$

e para a expressão do momento fletor $\mathrm{M}_{\mathrm{z}}$ :

$$
M_{z}=\frac{p}{2}\left(\ell x-x^{2}\right)
$$

A expressão diferencial a ser resolvida pelo método numérico de Runge-Kutta, é a seguinte:

$$
G J_{t} \phi^{\prime \prime}(x)+\frac{p^{2}}{4 E I_{y}}\left(\ell x-x^{2}\right)^{2} \phi(x)=0
$$

Utilizando o parâmetro $t$, onde $x=t . \ell$, tem-se:

$$
\frac{G J_{t}}{\ell^{2}} \phi^{\prime \prime}(t)+\frac{p^{2} \ell^{4}}{4 E I_{y}}\left(t-t^{2}\right)^{2} \phi(t)=0
$$

então:

$$
\phi^{\prime \prime}(t)+\frac{p^{2} \ell^{6}}{4 E I_{y} G J_{t}}\left(t-t^{2}\right)^{2} \phi(t)=0
$$

Chamando:

$$
\beta^{2}=\frac{p^{2} \ell^{6}}{4 E I_{y} G J_{t}}
$$

resulta a expressão final regente da instabilidade lateral:

$$
\phi^{\prime \prime}(t)=\beta^{2}\left(t-t^{2}\right)^{2} \phi(t)
$$

Utilizando o programa desenvolvido para a equação (2.35) em estudo, 
assumindo as condições de contorno para a aplicação do método de Runge-Kutta em problemas de auto-valor tem-se que o giro $\phi(0)=0$ nos apoios é nulo, e por simetria $\phi^{\prime}(\ell / 2)=0$. Deve-se adotar, por exemplo, $\phi^{\prime}(0)=1$ e calcular o valor de $\beta$ que satisfaz a condição de contorno conhecida no meio do vão.

$\mathrm{Na}$ aplicação do programa, para uma viga subdividida em 300 trechos, o valor de $\beta$ obtido foi 14,1550. Logo, a expressão de $p_{\text {crit }}$ resultante é:

$$
p_{c r t t}=28,31 \frac{\sqrt{G J_{t} E I_{y}}}{\ell^{3}}
$$

A partir de 100 trechos, os valores resultantes da carga crítica apresentaram-se muito próximos.

Nota-se a excelente aproximação entre os resultados obtidos pelos métodos de integração por séries de potência e o método numérico de Runge-Kutta. Entretanto, como o método de Runge-Kutta é facilmente programável, será preferível como solução neste estudo.

\subsection{INSTABILIDADE LATERAL DE VIGAS PROTENDIDAS EM SERVIÇO}

O estudo da instabilidade lateral das vigas protendidas durante a fase de serviço, desenvolvido por CATANIA \& COCCHI (1985) pelo método da energia potencial será descrito a seguir, para seções duplo $\mathrm{T}$ quaisquer apoiadas nas extremidades sobre vínculos indeformáveis de garfo. As armaduras de protensão são compostas de cabos retilíneos e paralelos ao eixo baricêntrico da viga de altura e seção constante.

Em primeira análise, a viga será analisada sob os efeitos isolados da protensão, e posteriormente sob ação conjunta com o carregamento vertical.

\subsubsection{CONSIDERAÇÕES GERAIS SOBRE A ENERGIA POTENCIAL TOTAL}

A expressão da energia potencial total pode ser apresentada pela adição de 3 parcelas: 


$$
E P T=U+T+T_{2}
$$

onde: $U=$ energia potencial dos esforços internos ou energia de deformação;

$\mathrm{T}=$ energia potencial dos esforços externos* (ou potencial propriamente dita);

$\mathrm{T}_{2}=$ energia potencial dos esforços internos de primeira ordem nas deformações

de segunda ordem;

* a energia potencial das cargas será sempre o trabalho realizado com sinal contrário.

$\mathrm{Na}$ definição de $\mathrm{U}$, utiliza-se os esforços internos de segunda ordem com as respectivas deformações. Em T, considera-se o potencial dos esforços aplicados em relação à posição original não deslocada da viga. A parcela $T_{2}$ completa as duas anteriores, na teoria de $2^{\mathrm{a}}$ ordem.

Esta interpretação e o modo de designar as diversas parcelas da energia é apenas uma entre as várias possíveis, e é a mesma utilizada em RACHID (1993).

A expressão da EPT ilustrada na publicação acima, para os mesmos tipos de carregamento indicados na equação (2.1), é:

$$
\begin{array}{rl}
E P T=\int_{I} & F\left(v_{D}, v_{D}^{\prime}, v_{D}^{\prime \prime}, w_{D}, w_{D}^{\prime \prime}, w_{D}^{\prime \prime \prime}, \phi, \phi^{\prime}, \phi^{\prime \prime}\right) d x \\
& -\sum\left(P_{y i} v_{D i}+P_{z i} w_{D i}+\frac{1}{2} P_{y i} e_{y i} \phi_{i}^{2}+\frac{1}{2} P_{z i} e_{z i} \phi_{i}^{2}\right) \\
& -\sum\left(M_{y i} w_{D i}^{\prime}+M_{z i} v_{D i}^{\prime}\right)-\sum B_{i} \phi_{i}^{\prime}
\end{array}
$$

onde o funcional F significa:

$$
\begin{aligned}
F=\frac{1}{2} & \left\{E I_{z} v_{D}^{\prime \prime 2}+E I_{y} w_{D}^{\prime \prime 2}+E J_{w} \phi^{\prime \prime 2}+G J_{t} \phi^{2}+\right. \\
& -2\left(p_{y} v_{D}+p_{z} w_{D}\right)-\left(p_{y} e_{y}+p_{z} e_{z}\right) \phi^{2}+ \\
& +N\left[i_{D}^{2} \phi^{\prime 2}+2\left(y_{D} w_{D}^{\prime}-z_{D} v_{D}^{\prime}\right) \phi^{\prime}+v_{D}^{\prime 2}+w_{D}^{\prime 2}\right]+ \\
& +2\left[M_{z}\left(K_{y}-y_{D}\right)+M_{y}\left(K_{z}-z_{D}\right)\right] \phi^{\prime 2}+ \\
& -2\left[M_{z} w_{D}^{\prime}-M_{y} v_{D}^{\prime}\right] \phi^{\prime}+\frac{B}{J_{w}} U_{w} \phi^{\prime 2}+ \\
& \left.-2\left(M_{z}^{\prime} w_{D}^{\prime}-M_{y}^{\prime} v_{D}^{\prime}\right) \phi\right\}
\end{aligned}
$$


Em RACHID (1993), o estudo da instabilidade lateral avalia os deslocamentos da seção pelo deslocamento do centro de torção $D$ : $v_{D}$ e $w_{D}$, em y e em $z$, respectivamente.

O método da energia, além de proporcionar a dedução das equações diferenciais da instabilidade a partir das considerações do cálculo variacional, é a base do desenvolvimento do processo de Ritz, no estudo da mudança de equilíbrio em teoria de $2^{\mathrm{a}}$ ordem.

É necessário escolher funções aproximadas para os deslocamentos que sejam representativas das condições de contorno e admitir funções para as elásticas, de forma que estas funções sejam dependentes de alguns parâmetros. A energia torna-se então função desses parâmetros, e com isso transforma o problema de encontrar o extremo da função EPT em um problema onde procura-se o extremo de uma função de um número finito de variáveis. Entretanto, apresenta o inconveniente da escolha das funções, as quais, para cada caso particular, devem satisfazer as condições de contorno do problema. Além disso as integrações podem tornar o problema trabalhoso.

Neste estudo, admitiu-se para as elásticas a forma senoidal e as integrais a serem desenvolvidas, na maioria das vezes encontram-se tabeladas.

\subsubsection{AÇÃO ISOLADA DA PROTENSÃO}

A viga protendida, ilustrada na figura 2.3 , está vinculada à torção nas extremidades e protendida com cabo retilíneo ou cabo resultante de excentricidade $\mathrm{e}_{0}$.

A configuração deformada é definida pelo deslocamento $\delta$ do centro de gravidade da seção segundo o eixo $\mathrm{z}$, e pela rotação $\phi$ da seção em torno do eixo longitudinal x. A força normal de protensão $\mathrm{N}_{\mathrm{p}}$ provoca reações internas representadas na figura 2.4, através das forças $\mathrm{f}_{0}(\mathrm{x})$ radiais distribuídas.

Segundo o diagrama de equilíbrio do cabo de protensão, onde os valores assumidos para o ângulo $\theta$ são muito pequenos $(\theta \sim \operatorname{sen} \theta \sim \operatorname{tg} \theta)$, tem-se: 


$$
\begin{gathered}
\int_{0}^{\theta} f_{0} r d \theta=2 N_{p} \operatorname{sen}\left(\frac{\theta}{2}\right)=2 N_{P} \frac{\theta}{2}=N_{P} \theta \\
\therefore N_{P}=f_{0} r
\end{gathered}
$$

Do elemento curvo submetido a uma pressão radial $\mathrm{f}_{0}(\mathrm{x})$, resulta:

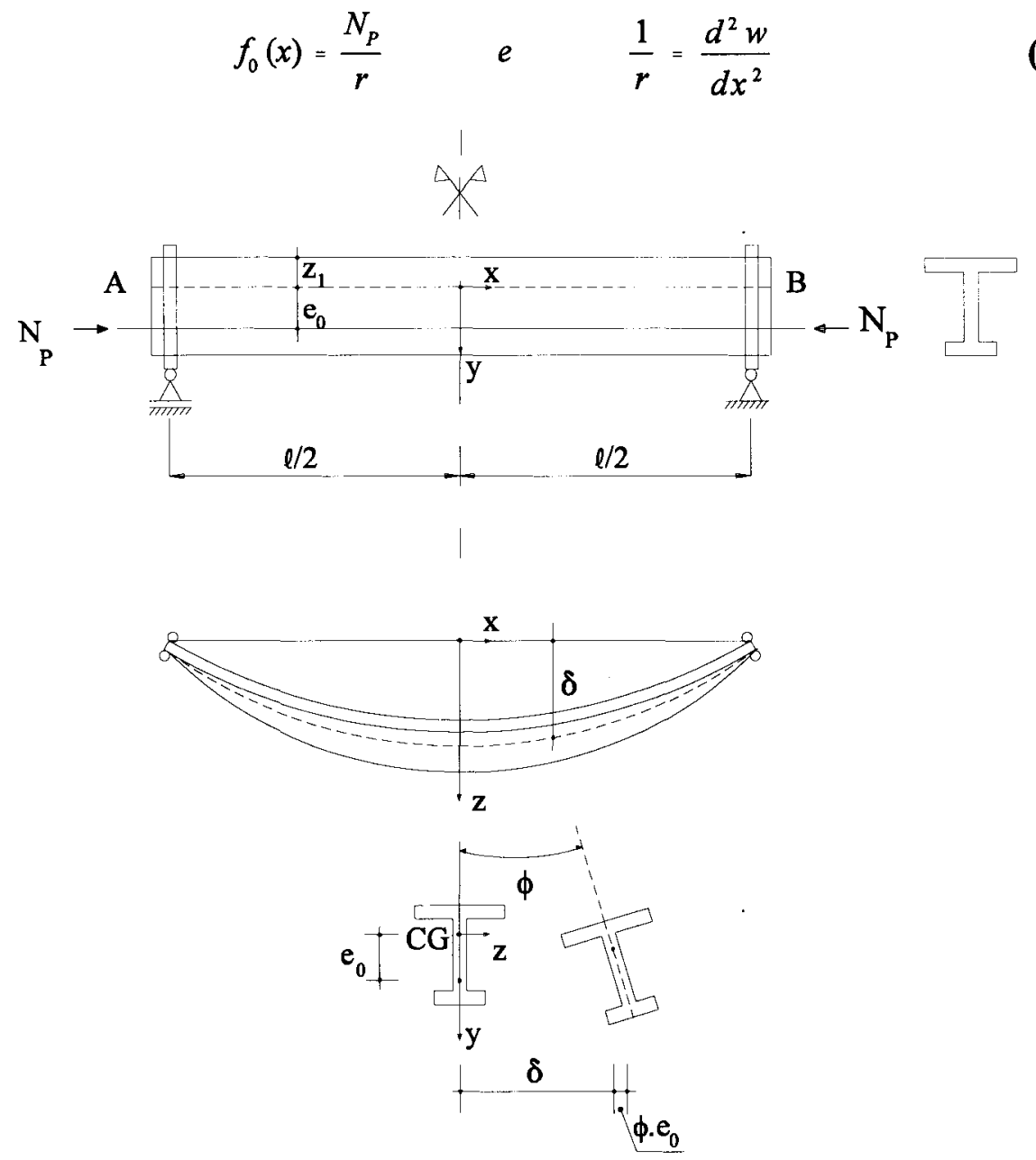

Figura 2.3 - Viga protendida de seção duplo T. [ CATANIA \& COCCHI (1985) ]

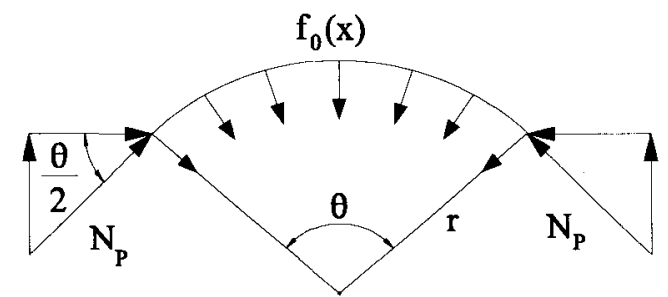

Figura 2.4 - Equilíbrio do cabo de protensão. 
Para um ponto genérico sob o cabo de protensão, o deslocamento $\mathrm{w}$ em z pode ser descrito por $\mathrm{w}=\delta(\mathrm{x})+\mathrm{e}_{0} \phi(\mathrm{x})$, onde $\delta$ é o deslocamento lateral em $\mathrm{z}$ do centro de gravidade. Assim, em $\mathrm{f}_{0}(\mathrm{x})$ tem-se:

$$
f_{0}(x)=N_{P} \frac{d^{2}\left(\delta+e_{0} \phi\right)}{d x^{2}}
$$

As tensões normal e cisalhante da viga protendida são:

$$
\begin{gathered}
\sigma_{x} \approx-\frac{N_{P}}{A}-\frac{N_{P} e_{0}}{I_{z}} y \\
\tau_{x y}=0
\end{gathered}
$$

$\mathrm{Na}$ energia de deformação U, não será considerada a contribuição da flexão vertical por não influenciar nos efeitos da flambagem. Os deslocamentos verticais são despreziveis frente aos deslocamentos laterais, pois a rigidez à flexão vertical é muito maior que a rigidez lateral e a rigidez por torção.

A expressão de $U$, por simetria, pode ser definida por:

$$
U=\int_{0}^{1 / 2} E I_{y} \delta^{\prime \prime 2}(x) d x+\int_{0}^{1 / 2} G J_{l} \phi^{\prime 2}(x) d x
$$

na qual CATANIA \& COCCHI (1985) não consideraram a contribuição das propriedades setoriais das seções delgadas.

O primeiro termo da expresão (2.43) representa a energia de deformação à flexão no plano horizontal, e o segundo refere-se à torção.

Sejam $\mathrm{u}, \mathrm{v}$, e w os deslocamentos segundo os eixos coordenados $\mathrm{x}, \mathrm{y} \mathrm{e} \mathrm{z}$, respectivamente.

$$
\begin{aligned}
& u=0 \\
& v=0 \\
& w=\delta(x)+y \phi(x)
\end{aligned}
$$

sendo nulo o giro por torção nos vínculos de garfo indeformável: $\phi(x)=0$ para $x= \pm \ell / 2$.

Em um ponto genérico ao longo de $\mathrm{x}$, para $\mathrm{y}=\mathrm{e}_{0}$, sob o cabo resultante 
tracionado ou sob a linha de ação da reação $\mathrm{f}_{0}(\mathrm{x})$, o deslocamento em y deste ponto, pode ser expresso por:

$$
e_{0}-\sqrt{e_{o}^{2}-\phi^{2} e_{0}^{2}}=e_{0}\left[1-\sqrt{1-\phi^{2}}\right] \sim e_{0}\left[1-\left(1-\frac{\phi^{2}}{2}\right)\right]=e_{0} \frac{\phi^{2}}{2}
$$

e segundo z:

$$
\delta+e_{0} \phi(x)
$$

A expressão da energia potencial $T_{2}$ dos esforços internos de $1^{\mathrm{a}}$ ordem nas deformações de $2^{\mathrm{a}}$ ordem é:

$$
T_{2}=\int_{V}\left(\sigma_{x} \varepsilon_{x}^{(2)}+\tau_{x y} \gamma_{x y}^{(2)}\right) d V
$$

onde $\varepsilon_{x}^{(2)} e \tau_{x y}^{\left({ }^{(2)}\right.}$ são relativas à $2^{a}$ ordem. Para $\tau_{x y}=0$, tem-se:

$$
T_{2}=\int_{V} \sigma_{x} \varepsilon_{x}^{(2)} d V
$$

A expressão da energia de deformação de $2^{\mathrm{a}}$ ordem resulta em:

$$
\begin{gathered}
\varepsilon_{x}=\varepsilon_{x}^{(1)}+\varepsilon_{x}^{(2)} \\
\varepsilon_{x}^{(2)}=\frac{1}{2}\left[\left(\frac{\partial v}{\partial x}\right)^{2}+\left(\frac{\partial w}{\partial x}\right)^{2}\right]=\frac{1}{2}\left[\frac{d}{d x}(\delta+y . \phi)\right]^{2}
\end{gathered}
$$

A parcela relativa à função $\mathrm{v}$ não será considerada, pois os deslocamentos verticais estão sendo desprezados em presença dos deslocamentos laterais.

$$
\varepsilon_{x}^{(2)}=\frac{1}{2}\left[\left(\frac{d \delta}{d x}\right)^{2}+2 y \frac{d \delta}{d x} \frac{d \phi}{d x}+y^{2}\left(\frac{d \phi}{d x}\right)^{2}\right]
$$

Então, substituindo $\sigma_{\mathrm{x}} \mathrm{e} \varepsilon_{\mathrm{x}}^{(2)}$, tem-se:

$$
T_{2}=\int_{0}^{\ell / 2} \int_{A}-N_{P}\left(\frac{1}{A}+\frac{e_{0}}{I_{z}} y\right) \cdot\left[\left(\frac{d \delta}{d x}\right)^{2}+2 y \frac{d \delta}{d x} \frac{d \phi}{d x}+y^{2}\left(\frac{d \phi}{d x}\right)^{2}\right] d A d x
$$

sendo válidas as igualdades definidas em (2.52): 


$$
\int_{A} y d A=0 \quad \int_{A} y^{3} d A=0 \quad \int_{A} y^{2} d A=I_{z}=i_{z}^{2} A
$$

onde $\mathrm{i}_{\mathrm{z}}$ é o raio de giração.

Assim, a expressão da energia potencial $\mathrm{T}_{2}$ é:

$$
T_{2}=-N_{P} \int_{0}^{u / 2}\left[\left(\frac{d \delta}{d x}\right)^{2}+i_{x}^{2}\left(\frac{d \phi}{d x}\right)^{2}+2 e_{0} \frac{d \delta}{d x} \frac{d \phi}{d x}\right] d x
$$

Entretanto, conforme definição de integração por partes, tem-se:

$$
\int_{0}^{2 / 2} \frac{d \delta}{d x} \frac{d \phi}{d x} d x=\left[\frac{d \delta}{d x} \phi\right]_{0}^{\ell / 2}-\int_{0}^{d / 2} \phi \frac{d^{2} \delta}{d x^{2}} d x=-\int_{0}^{1 / 2} \phi \frac{d^{2} \delta}{d x^{2}} d x
$$

onde $\delta^{\prime}(0)=0$ (giro lateral por flexão na seção de simetria) e $\phi(\ell / 2)=0$ (giro por torção nos apoios). A origem do eixo longitudinal $\mathrm{x}$ encontra-se na seção de simetria da viga.

A expressão resultante da energia $T_{2}$ é definida por:

$$
T_{2}=-N_{P} \int_{0}^{v / 2}\left[\left(\frac{d \delta(x)}{d x}\right)^{2}+i_{z}^{2}\left(\frac{d \phi(x)}{d x}\right)^{2}-2 e_{0} \phi \frac{d^{2} \delta(x)}{d x^{2}}\right] d x
$$

A energia potencial dos esforços externos $\mathrm{T}$ ou energia potencial propriamente dita é descrita pelo trabalho realizado pela força de protensão.

$$
T=-\frac{1}{2} \int_{-v^{2}}^{\ell / 2} f_{0}(x)\left[\delta(x)+e_{0} \phi(x)\right] d x
$$

Substituindo $\mathrm{f}_{0}(\mathrm{x})$ da equação (2.40), para $\mathrm{N}_{\mathrm{p}}$ constante ao longo de $\mathrm{x}$, tem-se:

$$
T=-\frac{1}{2} \int_{-1 / 2}^{u / 2} N_{P} \frac{d^{2}}{d x^{2}}\left(\delta+e_{0} \phi\right)\left[\delta+e_{0} \phi\right] d x
$$

onde,

$$
\frac{d^{2}\left(\delta+e_{0} \phi\right)}{d x^{2}}=\frac{d^{2} \delta}{d x^{2}}+e_{0} \frac{d^{2} \phi}{d x^{2}}
$$


Então, o trabalho das forças externas T, vale:

$$
T=-N_{P} \int_{0}^{1 / 2}\left[\frac{d^{2} \delta}{d x^{2}} \delta+\delta e_{0} \frac{d^{2} \phi}{d x^{2}}+e_{0} \phi \frac{d^{2} \delta}{d x^{2}}+e_{0}^{2} \phi \frac{d^{2} \phi}{d x^{2}}\right] d x
$$

A equação da energia potencial total para ação única da força de protensão é:

$$
\begin{aligned}
E P T= & \int_{0}^{\ell / 2} E I_{y} \delta^{\prime \prime} d x+\int_{0}^{\ell / 2} G J_{t} \phi^{\prime 2} d x+ \\
& -N_{P} \int_{0}^{\ell / 2}\left(\delta e_{0} \frac{d^{2} \phi}{d x^{2}}+e_{0}^{2} \phi \frac{d^{2} \phi}{d x^{2}}+i_{z}^{2}\left(\frac{d \phi}{d x}\right)^{2}+\right. \\
& \left.-e_{0} \phi \frac{d^{2} \delta}{d x^{2}}+\frac{d^{2} \delta}{d x^{2}} \delta+\left(\frac{d \delta}{d x}\right)^{2}\right) d x
\end{aligned}
$$

de forma que a expressão final de EPT pode ser reescrita por:

$$
\begin{aligned}
E P T= & \int_{0}^{\ell / 2} E I_{y} \delta^{\prime \prime 2}(x) d x+\int_{0}^{\ell / 2} G J_{l} \phi^{\prime 2}(x) d x+ \\
& -N_{P} \int_{0}^{\ell / 2}\left(\delta e_{0} \frac{d^{2} \phi}{d x^{2}}+e_{0}^{2} \phi \frac{d^{2} \phi}{d x^{2}}+i_{z}^{2}\left(\frac{d \phi}{d x}\right)^{2}-e_{0} \phi \frac{d^{2} \delta}{d x^{2}}\right) d x
\end{aligned}
$$

para o resultado da integral:

$$
\int_{0}^{1 / 2} \delta^{\prime 2}(x)=\left.\delta \cdot \delta^{\prime}\right|_{0} ^{\ell / 2}-\int_{0}^{1 / 2} \delta \cdot \delta^{\prime \prime} d x=-\int_{0}^{\ell / 2} \delta(x) \cdot \delta^{\prime \prime}(x) d x
$$

onde as condições de contorno são: $\delta^{\prime}(0)=0$ e $\delta(\ell / 2)=0$.

Atribuindo as equações definidas em (2.63) para elásticas, de modo que satisfaçam as condições de contorno e representem o primeiro modo de flambagem, tem-se:

$$
\delta=a \cos \frac{\pi x}{\ell} \quad \phi=b \cos \frac{\pi x}{\ell}
$$

para $\mathrm{x}=0, \delta=\mathrm{a}$ e $\phi=\mathrm{b}$, pois na seção central ocorre deslocamento lateral e giro por torção, e em $\mathrm{x}=\ell / 2$, nos apoios, $\delta=0$ e $\phi=0$, visto que os vínculos de garfo são rígidos.

Procede-se derivando as funções das elásticas, e em seguida, substitui-se $\delta, \delta "$, $\phi, \phi^{\prime}$ e $\phi^{\prime \prime}$ na expressão de EPT. 
A expressão resultante da energia potencial total para as elásticas assumidas é:

$$
E P T=E I_{y} \frac{\pi^{4}}{4 \ell^{3}} a^{2}+G J_{t} \frac{\pi^{2}}{4 \ell} b^{2}+N_{P} \frac{\pi^{2}}{4 \ell}\left(e_{0}^{2}-i_{z}^{2}\right) b^{2}
$$

sendo o resultado de algumas integrais comuns:

$$
\int_{0}^{\ell / 2} \cos ^{2} \frac{\pi x}{\ell} d x=\int_{0}^{\ell / 2} \operatorname{sen}^{2} \frac{\pi x}{\ell} d x=\frac{\ell}{4}
$$

Os extremos da função EPT devem ser procurados nos extremos dos parâmetros das funções: as funções $\delta$ e $\phi$ estão associadas aos parâmetros a e b. Para a determinação da carga crítica devem ser simultaneamente satisfeitas as condições abaixo relacionadas:

$$
\frac{\partial E P T}{\partial a}=0 \quad e \quad \frac{\partial E P T}{\partial b}=0
$$

Assim:

$$
\begin{aligned}
& \frac{\partial E P T}{\partial a}=2 E I_{y} \frac{\pi^{4}}{4 \ell^{3}} a=0 \\
& \frac{\partial E P T}{\partial b}=2 G J_{t} \frac{\pi^{2}}{4 \ell} b+2 N_{P} \frac{\pi^{2}}{4 \ell}\left(e_{0}^{2}-i_{z}^{2}\right) b=0
\end{aligned}
$$

O limite de instabilidade é definido quando o determinante do sistema expresso em (2.67) for nulo, ou seja, solução diferente da trivial.

$$
\left|\begin{array}{cc}
E I_{y} \frac{\pi^{4}}{2 \ell^{3}} & 0 \\
0 & \frac{\pi^{2}}{2 \ell}\left[G J_{t}+N_{P}\left(e_{0}^{2}-i_{z}^{2}\right)\right]
\end{array}\right|=0
$$

Desta forma:

$$
E I_{y} \frac{\pi^{6}}{4 \ell^{4}}\left[G J_{t}+N_{P}\left(e_{0}^{2}-i_{z}^{2}\right)\right]=0
$$

A expressão da força de protensão para as análises da instabilidade lateral é: 


$$
N_{P}=-\frac{G J_{t}}{\left(e_{0}^{2}-i_{z}^{2}\right)}
$$

Esta expressão significa que existe um valor crítico para a força normal $\mathrm{N}_{\mathrm{P}}$ de protensão, sob sua ação única dada pela expressão (2.69), e que depende diretamente do resultado da diferença $\left(\mathrm{e}_{0}{ }^{2}-\mathrm{i}_{\mathrm{z}}{ }^{2}\right)$.

No desenvolvimento da expressão da força crítica de protensão, para $N_{P}$ positivo, a força normal é de compressão.

\subsubsection{AÇÃO CONJUNTA DO CARREGAMENTO VERTICAL E DA FORÇA DE PROTENSÃO}

Para o carregamento externo aplicado ilustrado na figura 2.5, e considerando os efeitos já avaliados para a protensão, tem-se a expressão de $T+T_{2}$, conforme desenvolvido no item 2.4.1. Assim:

$$
\begin{aligned}
T+T_{2}= & N_{P} \int_{0}^{1 / 2}\left[e_{0} \delta(x) \phi^{\prime \prime}(x)+e_{0}^{2} \phi(x) \phi^{\prime \prime}(x)+i_{z}^{2} \phi^{\prime 2}(x)-e_{0} \phi(x) \delta^{\prime \prime}(x)\right] d x+ \\
& -d^{\prime / 2} \int_{0}^{1 / 2} p_{z}(x) \phi^{2}(x) d x
\end{aligned}
$$

onde a última parcela, representativa do trabalho do carregamento vertical, é o trabalho realizado pelo momento provocado por $\mathrm{p}_{z}$ em relação ao centro de torção vezes o giro.

A expressão da energia potencial total é:

$$
\begin{aligned}
E P T & =\int_{0}^{\ell / 2} E I_{y} \delta^{\prime \prime 2}(x) d x+\int_{0}^{\ell / 2} G J_{\iota} \phi^{\prime 2}(x) d x+ \\
& -N_{P} \int_{0}^{\ell / 2}\left[e_{0} \delta(x) \phi^{\prime \prime}(x)+e_{0}^{2} \phi(x) \phi^{\prime \prime}(x)+i_{z}^{2} \phi^{\prime 2}(x)-e_{0} \phi(x) \delta^{\prime \prime}(x)\right] d x+ \\
& -d \cdot \int_{0}^{\ell / 2} p_{z}(x) \phi^{2}(x) d x
\end{aligned}
$$

Para a definição de um carregamento vertical qualquer aplicado a uma distância $d^{*}$ do centro de torção da seção, CATANIA \& COCCHI (1985) utilizaram as séries de 


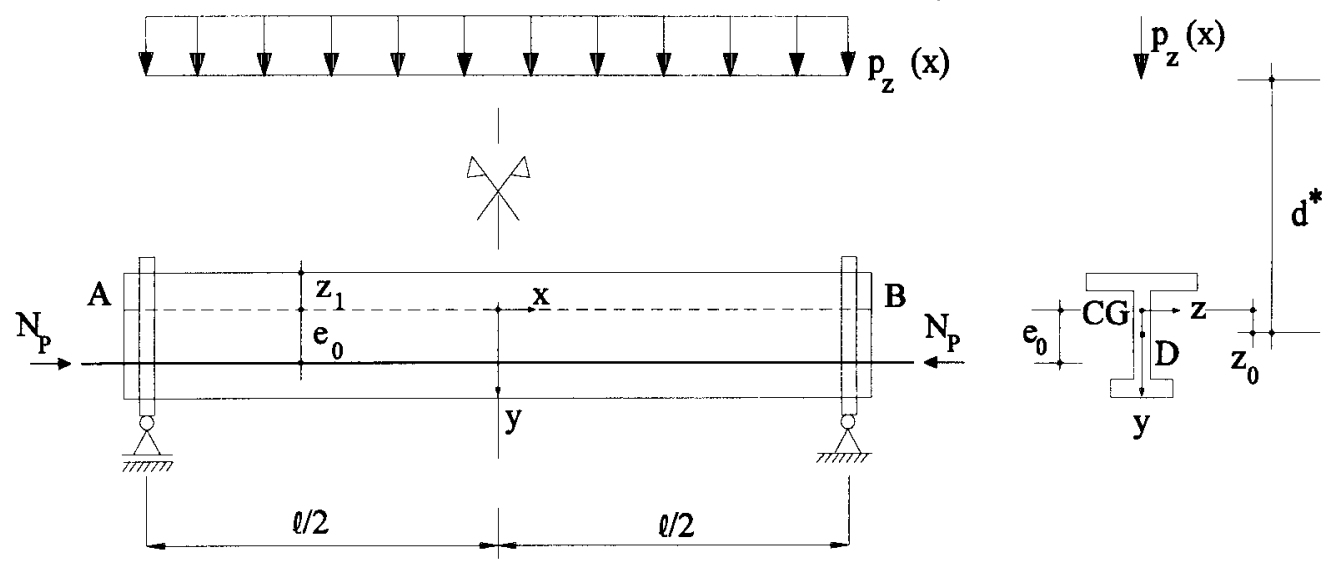

Figura 2.5 - Viga protendida biapoiada sob carregamento vertical.

[ CATANIA \& COCCHI (1985)]

Fourier.

$$
p_{z}(x)=p_{0}+\sum_{m=1}^{\infty}\left(p_{1 m} \operatorname{sen} \frac{m \pi x}{\ell}+p_{2 m} \cos \frac{m \pi x}{\ell}\right)
$$

de forma que, interrompendo a expresssão da série para $m=1$, desenvolvendo a última integral da expressão (2.71) para a série de $\mathrm{p}_{z} \mathrm{e}$, substituindo a função $\phi$ por sua expressão definida em (2.63), tem-se:

$$
\begin{aligned}
&-d^{*} \int_{0}^{\ell / 2} p_{z}(x) \phi^{2}(x) d x=-d^{\cdot}\left[\int_{0}^{\ell / 2} b^{2} p_{0}^{2} \cos ^{2} \frac{\pi x}{\ell} d x+\right. \\
&\left.+\int_{0}^{\ell / 2} b^{2} p_{1}^{2} \operatorname{sen} \frac{\pi x}{\ell} \cos ^{2} \frac{\pi x}{\ell} d x+\int_{0}^{\ell / 2} b^{2} p_{2}^{2} \cos ^{3} \frac{\pi x}{\ell} d x\right]= \\
&=-d^{*} b^{2} \frac{\ell}{\pi}\left[p_{o} \frac{\pi}{4}+p_{1} \frac{1}{3}+p_{2} \frac{2}{3}\right]
\end{aligned}
$$

Os coeficientes da série de Fourier podem ser definidos no intervalo de - $/ / 2$ à $\ell / 2:$ 


$$
\begin{aligned}
& p_{0}=p \| \text { carga constante } \\
& p_{1}=\frac{2}{\ell} \int_{-/ 2}^{\ell / 2} p_{z}(x) \operatorname{sen} \frac{\pi x}{\ell} d x \\
& p_{2}=\frac{2}{\ell} \int_{-/ 2}^{\ell / 2} p_{z}(x) \cos \frac{\pi x}{\ell} d x
\end{aligned}
$$

e por simplicidade designa-se $\mathrm{p}^{*}$ por:

$$
p^{*}=\frac{\pi}{4} p_{0}+\frac{1}{3} p_{1}+\frac{2}{3} p_{2}
$$

Para carga uniformemente distribuída: $\mathrm{p}_{1}=\mathrm{p}_{2}=0$; para carregamento simétrico em relação ao eixo de simetria: $\mathrm{p}_{2}=0$ e para carregamento antissimétrico: $\mathrm{p}_{0}=\mathrm{p}_{1}=0$.

A expressão final de EPT para as elásticas assumidas resulta em:

$$
\begin{aligned}
E P T=E I_{y} \frac{\pi^{4}}{4 \ell^{3}} a^{2}+ & G J_{t} \frac{\pi^{2}}{4 \ell} b^{2}+N_{P} \frac{\pi^{2}}{4 \ell}\left(e_{0}^{2}-i_{z}^{2}\right) b^{2}+ \\
& -d^{\cdot} b^{2} \frac{\ell}{\pi}\left(\frac{\pi}{4} p_{0}+\frac{1}{3} p_{1}+\frac{2}{3} p_{2}\right)
\end{aligned}
$$

Da mesma forma, para determinar a carga crítica, deve-se proceder realizando as derivadas parciais da EPT em relação aos parâmetros a e b da deformada.

$O$ valor crítico de instabilidade lateral depende da correlação entre $N_{P}$ e $p^{*}$, para que posto $n=N_{p} / p^{*}$, o sistema de equações possa ser escrito.

$$
\begin{aligned}
& \frac{\partial E P T}{\partial a}=E I_{y} \frac{\pi^{4}}{2 \ell^{3}} a=0 \\
& \frac{\partial E P T}{\partial b}=G J_{t} \frac{\pi^{2}}{2 \ell} b+N_{P} \frac{\pi^{2}}{2 \ell}\left(e_{0}^{2}-i_{z}^{2}\right) b-2 d \cdot \frac{\ell}{\pi} b\left(\frac{\pi}{4} p_{0}+\frac{1}{3} p_{1}+\frac{2}{3} p_{2}\right)=0
\end{aligned}
$$

Substituindo n na expressão e fazendo nulo o determinante de forma a existir solução para a e b diferente de zero, tem-se:

$$
\left|\begin{array}{cc}
E I_{y} \frac{\pi^{4}}{2 \ell^{3}} & 0 \\
0 & \frac{\pi^{2}}{2 \ell}\left[G J_{t}+p^{*} n\left(e_{0}^{2}-i_{z}^{2}\right)\right]-2 d \cdot p \cdot \frac{\ell}{\pi}
\end{array}\right|=0
$$


A expressão da carga crítica para o determinante igual a zero resulta em:

$$
p_{c r t t}=-\frac{G J_{t}}{n\left(e_{0}^{2}-i_{z}^{2}\right)-\frac{4 d \cdot l^{2}}{\pi^{3}}}
$$

Por tentativas deve-se ter para um dado $\mathrm{n}$, um valor para $\mathrm{p}_{\text {crit }}{ }^{*}$ de tal forma que a força de protensão $\mathrm{N}_{\mathrm{p}}$ seja dada por $\mathrm{N}_{\mathrm{p}} / \mathrm{p}_{\text {crit }}^{*}$ n. As tentativas terminam quando esta última condição for satisfeita.

\subsection{INSTABILIDADE LATERAL DE VIGAS EM SERVIÇO SOBRE APOIOS DEFORMÁVEIS}

No estudo da instabilidade lateral de vigas, admitindo ligações deformáveis à torção, entende-se que estes vínculos apresentando graus de liberdade, aumentam os riscos de perda de estabilidade em vigas esbeltas.

Neste item, as deformabilidades serão tratadas como conhecidas. Para a determinação da deformabilidade à torção das ligações, deve-se ter um modelo analítico que permita o cálculo, e que se possível, apresente comprovação experimental.

O comportamento das vigas sobre ligações deformáveis à torção, neste trabalho vínculos de garfo, pode ser avaliado através de molas dispostas de forma conveniente a introduzir a deformabilidade.

Uma analogia será feita ao estudo da estabilidade das vigas em suspensão desenvolvido por CATANIA \& COCCHI (1985), a ser descrito no capítulo 3.

Para vigas sobre apoios deformáveis, $\mathrm{K}_{\phi}$ é a constante de mola ilustrada na figura $2.6 \mathrm{e} \lambda$ é a deformabilidade à torção da ligação. Assim:

$$
M_{t}=-K_{\phi} \phi \quad ; \quad K_{\phi}=\frac{1}{\lambda}
$$




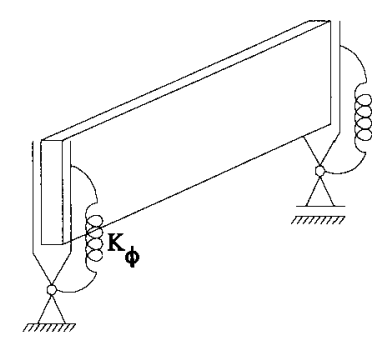

Figura 2.6 - Viga apoiada sobre vínculo de garfo deformável à torção.

\subsubsection{VIGA SOBRE APOIOS DEFORMÁVEIS À TORÇÃO}

O equacionamento desenvolvido referente à vigas de seção retangular, pode ser também elaborado para outros tipos de seções. A deformabilidade dos apoios é introduzida ao estudo da instabilidade através das condições de contorno. Porém, a definição de um mecanismo representativo depende das ferramentas com as quais o modelo será projetado, bem como da complexidade da ligação.

Assumindo uma ligação deformável, resultam que os deslocamentos laterais e o giro por torção nos apoios não é nulo.

A resolução da equação regente da instabilidade será desenvolvida por séries de potência, aproveitando o equacionamento desenvolvido por CATANIA \& COCCHI (1985) para a fase transitória.

Para a utilização de um outro método, como o processo de Ritz, no estudo da energia potencial as elásticas devem ser representadas, por exemplo, por uma função seno ou cosseno, dependendo da disposição dos eixos coordenados e da configuração da deformada, acrescidos de uma constante. Esta constante irá representar o giro inicial permitido pela ligação.

A figura 2.7 ilustra a viga de seção retangular apoiada sobre garfos deformáveis à torção nas extremidades. $\mathrm{O}$ carregamento é considerado linearmente distribuído na linha do CG. 


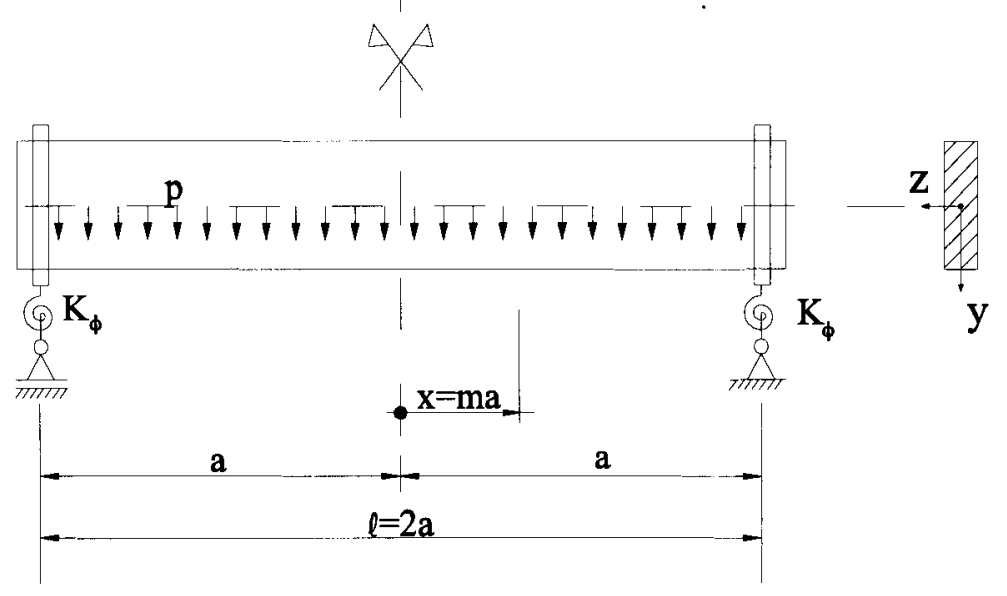

Figura 2.7 - Viga em serviço sobre apoios deformáveis à torção.

A expressão do momento fletor $\mathrm{M}_{\mathrm{z}}$ em uma seção qualquer, para a origem do eixo longitudinal na seção de simetria da viga, é:

$$
M_{z}(x)=\frac{p \ell}{2}\left(\frac{\ell}{2}-x\right)-\frac{p}{2}\left(\frac{\ell}{2}-x\right)^{2}
$$

Utilizando a variável auxiliar $\mathrm{m}=2 \mathrm{x} / \ell$ onde $\ell=2 \mathrm{a}$, tem-se:

$$
M_{z}(m)=\frac{p a^{2}}{2}\left(1-m^{2}\right)
$$

A equação regente da instabilidade lateral pode ser escrita como segue:

$$
\phi^{\prime \prime}(x)+\frac{M_{z}^{2}(x)}{E I_{y} G J_{t}} \phi(x)=0
$$

Reescrevendo a equação diferencial acima, em função da variável auxiliar m, e substituindo a expressão de $M_{z}(m)$, tem-se:

$$
\phi^{\prime \prime}(m)+\frac{p^{2} a^{6}}{4 E I_{y} G J_{t}}\left(1-m^{2}\right)^{2} \phi(m)=0
$$

onde deve-se lembrar que $\mathrm{dx} / \mathrm{dm}=\mathrm{a}$, e $\alpha$ pode novamente ser definido como: 


$$
\alpha=\frac{p^{2} a^{6}}{4 E I_{y} G J_{t}}
$$

Enfim, a expressão (2.84) resulta em:

$$
\phi^{\prime \prime}(m)+\alpha\left(1-m^{2}\right)^{2} \phi(m)=0
$$

Resolvendo a equação diferencial por integração de séries de potência, conforme feito para apoios indeformáveis no item 2.3.1, tem-se os procedimentos similares até a definição das condições de contorno.

Como a seção retangular apenas tem torção livre e o giro nos apoios não é nulo, a igualdade abaixo pode ser expressa:

$$
M_{t}=M_{\imath}=G J_{t} \phi^{\prime}=-K_{\phi} \cdot \phi
$$

onde o sinal negativo vem da concordância entre a convenção de sinais adotada para o momento de torção e o respectivo deslocamento angular.

Então:

$$
G J_{t} \phi_{x= \pm a}^{\prime}=-K_{\phi} \phi_{x= \pm a}^{\mid}
$$

Sendo $x=m a$ e $d x / m=a$, pode-se reescrever a equação $(2.87)$ para $m=1$ :

$$
\frac{G J_{t}}{a} \phi^{\prime}(m=1)=-K_{\phi} \phi(m=1)
$$

Aplicando as séries desenvolvidas para $\phi(\mathrm{m})$ e $\phi^{\prime}(\mathrm{m})$ para $\mathrm{m}=1$ e desprezando as parcelas com expoentes maiores que 2 , tem-se de (2.9) e (2.13):

$$
\begin{aligned}
& \phi(1)=A_{0}\left[1-\frac{11}{30} \alpha+\frac{6617}{415800} \alpha^{2}\right] \\
& \phi^{\prime}(1)=A_{0}\left[-\frac{8}{15} \alpha+\frac{356}{10395} \alpha^{2}\right]
\end{aligned}
$$

Para o cálculo da carga crítica, é preciso substituir na condição de contorno que considera a deformabilidade à torção do vínculo, as expressões $\phi(1)$ e $\phi^{\prime}(1)$.

Então: 


$$
\begin{aligned}
\frac{G J_{t}}{a} \phi^{\prime}(1) & =-K_{\phi} \phi(1) \\
\frac{G J_{t}}{a} A_{0}\left[-\frac{8}{15} \alpha+\frac{356}{10395} \alpha^{2}\right] & =-K_{\phi} A_{0}\left[1-\frac{11}{30} \alpha+\frac{6617}{415800} \alpha^{2}\right]
\end{aligned}
$$

e isolando os termos conhecidos, pode-se escrever:

$$
\frac{K_{\phi} \ell}{2 G J_{t}}=f(\alpha)=\frac{\frac{8}{15} \alpha-\frac{356}{10395} \alpha^{2}}{1-\frac{11}{30} \alpha+\frac{6617}{415800} \alpha^{2}}
$$

Para o valor de conhecido da função $f(\alpha)$, tira-se da expressão acima o valor da incógnita $\alpha$. Substituindo $\alpha$ na expressão (2.92), tem-se o valor da carga crítica.

$$
p_{c r t t}=16 \sqrt{\alpha_{c r t t}} \frac{\sqrt{E I_{y} G J_{t}}}{\ell^{3}}
$$

Analisando a expressão (2.91), para rigidez infinita $\mathrm{K}_{\phi} \rightarrow \infty$, a função $\mathrm{f}(\alpha) \rightarrow \infty$, ou seja, o denominador da referida equação tende a zero:

$$
1-\frac{11}{30} \alpha+\frac{6617}{415800} \alpha^{2}=0
$$

então, $\alpha_{\text {crit }}=3,16$, o que retorna à expressão para apoios indeformáveis.

O gráfico da figura 2.8 ilustra a expressão da função $f(\alpha)$, de modo a facilitar o cálculo da carga uniforme distribuída crítica, a partir do valor da constante de mola $K_{\phi}$ da ligação, do comprimento $\ell$ e da rigidez à torção $G_{\mathrm{t}}$ da viga de seção retangular.

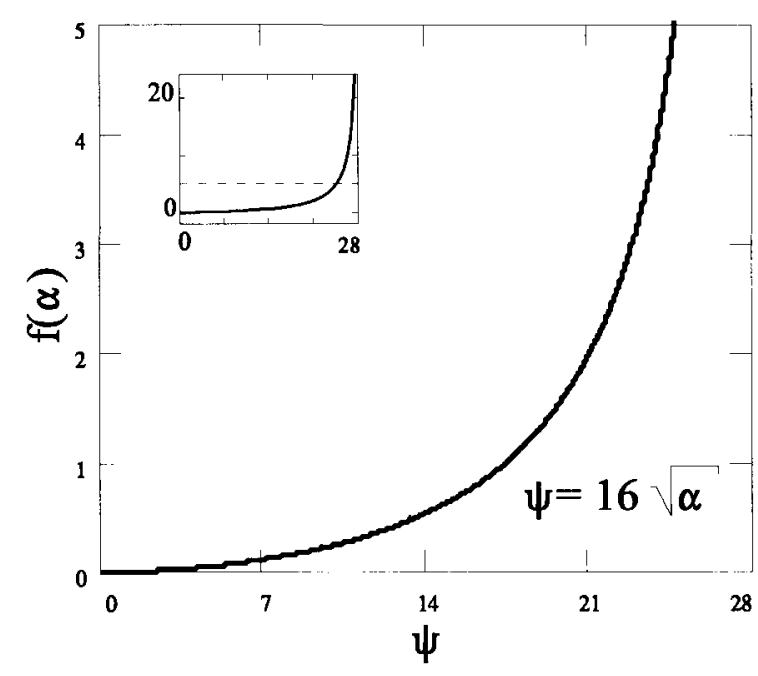

Figura 2.8 - Gráfico da função $f(\alpha)$ em função de $\psi$ 


\section{INSTABILIDADE LATERAL DE VIGAS DURANTE A FASE TRANSITÓRIA DE SUSPENSÃO}

\subsection{PRELIMINARES}

A pré-moldagem introduz ao dimensionamento dos elementos estruturais uma fase incomum ao projeto de estruturas de concreto armado moldado no local.

A fase transitória, dentre outras etapas, inclui a suspensão para manuseio no canteiro da fábrica, ou para a disposição definitiva do elemento pré-moldado na estrutura em montagem.

Durante a fase transitória, o carregamento externo é nulo, e à primeira vista pode-se supô-la de menor importância.

Entretanto, a fase transitória de suspensão é muitas vezes crítica em relação à estabilidade lateral. Isto se deve aos graus de liberdade apresentados pelas vinculações temporárias e ao uso atualmente frequente de vigas esbeltas de longos vãos, geralmente protendidas, como as vigas de pontes. Também nas vigas de seções normalmente delgadas, como as vigas de argamassa armada e os perfis metálicos durante a montagem 
de estruturas mistas, problemas por excesso de deformação lateral são comuns.

A presença de imperfeições no processo de produção, como por exemplo, pequenos desvios no ponto de aplicação da protensão, no posicionamento dos ganchos para acoplar os cabos de elevação, contribui para a perda de estabilidade lateral. As excentricidades apresentadas na produção serão consideradas no capítulo 4 , segundo indicações do PCI Design Handbook $3^{\text {a }}$ edição e de MAST (1993).

A importância de assegurar a estabilidade durante a montagem das estruturas pré-moldadas não é apenas fundamental do ponto de vista da integridade do elemento, mas também por possibilitar acidentes no trabalho.

Neste capítulo será abordado o estudo da instabilidade lateral das vigas prémoldadas em fase de içamento, sem considerar a presença das imperfeições construtivas e de montagem.

O processo de integração por séries de potência foi aplicado por CATANIA \& COCCHI (1985) às equações diferenciais regentes da instabilidade lateral, para a determinação da carga crítica que provoca mudança de equilíbrio. O estudo da instabilidade durante a fase de suspensão, para várias disposições dos cabos de içamento, encontra-se descrito neste capítulo. Para as vigas protendidas de seção duplo T quaisquer, o estudo do equilíbrio será apresentado pelo desenvolvimento do método da energia potencial.

Desenvolveu-se neste trabalho, quatro programas que automatizam o método numérico de Runge-Kutta, na resolução do sistema de equações diferenciais regente da instabilidade lateral. $\mathrm{O}$ estudo trata de vigas de seção retangular e seções duplo $\mathrm{T}$ simétrico, na suspensão por cabos retos e inclinados. Os cabos dispostos nas seções de extremidade da viga, na sua face superior, estão contidos no plano longitudinal vertical de simetria. Os programas foram definidos de forma a permitirem a determinação tanto da carga crítica, relativa a um carregamento linear uniforme distribuído na linha do CG, como do comprimento crítico para o mesmo tipo de carregamento. 


\subsection{ESTUDO DA INSTABILIDADE UTILIZANDO INTEGRAÇÃO POR SÉRIES DE POTÊNCIA}

O cálculo da carga crítica, a partir da resolução da equação diferencial pelo método de integração por séries de potência, foi desenvolvido para a suspensão por cabos oblíquos fixos nas seções de extremidade, considerando a deformabilidade dos cabos, segundo CATANIA \& COCCHI (1985).

Em vigas de seção delgada, para qualquer uma das disposições dos cabos de içamento avaliadas, o estudo da carga crítica aplica-se à seções retangulares, seções cruz e seções $T$, quaisquer seções duplamente simétricas, ou melhor, às seções que não apresentam características setoriais.

Porém, os autores acima citados, não fazem nenhuma restrição à utilização do estudo desenvolvido para a fase transitória de suspensão, a determinados tipos de seções transversais. Esta observação baseia-se no fato de que, todo o estudo formulado partiu da expressão diferencial relativa a uma viga sem características setoriais, como a de seção retangular, submetida a carregamento uniformemente distribuído no eixo baricêntrico.

\subsubsection{CABOS OBLÍQUOS}

Seja uma viga sujeita à ação de seu peso-próprio, cujos pontos de içamento estão dispostos a uma distância $e$ acima do centro de gravidade, conforme ilustrado na figura 3.1 .

Um pequeno giro $\phi$ da posição vertical na seção do apoio, resulta em um momento de torção na viga igual a -p€eф/2, esquematizado na figura 3.2. Pode-se denominar como constante elástica $\mathrm{R}_{\mathrm{x}}$ o seguinte valor:

$$
R_{x}=\frac{1}{2} p \ell e
$$

CATANIA \& COCCHI (1985) indicam a expressão (3.2) para o cálculo da distância $e$, em casos similares e aproximáveis aos da figura 3.1 . 


$$
e=d+\frac{H}{1+\frac{p \ell \cos \beta}{4 E_{a} A_{a} \operatorname{sen}^{3} \gamma \operatorname{sen}^{2} \beta}}
$$

onde:.$_{\mathrm{a}}$ e $A_{\mathrm{a}}$ são, repectivamente, o módulo de elasticidade e área da seção transversal do cabo de suspensão;

. $\gamma$ e $\beta$ são os ângulos indicados na figura 3.1;

. d é a distância entre o CG da viga e o ponto de fixação dos cabos, conforme ilustra a figura $3.1 \mathrm{c}$, ou equivale à altura da seção, como indicado na figura $3.1 \mathrm{~b}$.

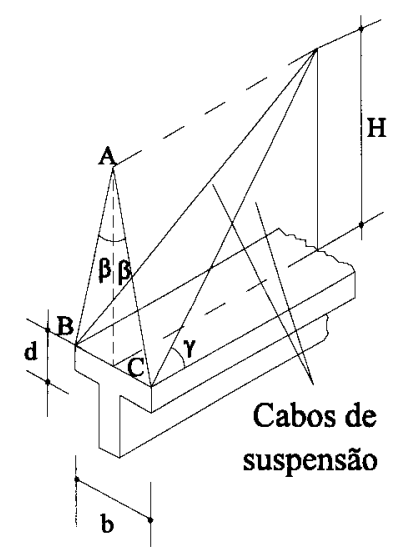

(a)

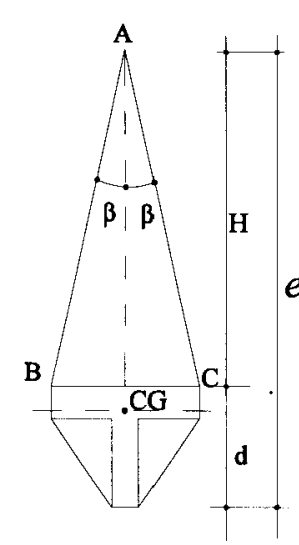

(b)

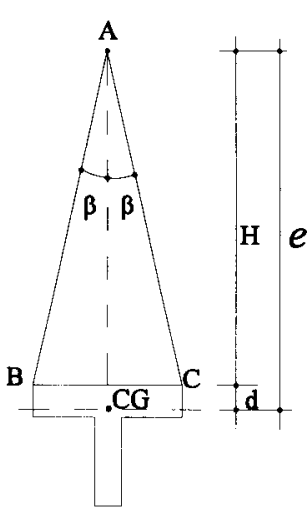

(c)

Figura 3.1 - Excentricidade $e$. CATANIA \& COCCHI (1985).

Uma análise mais detalhada da expressão (3.2) permite limitar sua utilização. Primeiramente, para a suspensão em 2 pontos, um em cada extremidade, o valor do ângulo de inclinação $\gamma$ dos cabos não influencia o valor da carga crítica. Assim, para $\beta$ tendendo a zero e $\gamma$ qualquer, tem-se $e=\mathrm{d}$. Isto significa que a componente normal resultante da ação inclinada da força de suspensão, a qual deve aumentar a instabilidade da suspensão, não está sendo avaliada. Alguns exemplos apresentados no capítulo 5 , mostram numericamente, a influência do ângulo de inclinação dos cabos no carrregamento crítico.

Também para a situação onde $\beta=\gamma=90^{\circ}$, o significado físico de $H$ não é esclarecido, sendo $e$ igual à soma $\mathrm{d}+\mathrm{H}$.

Em suma, as situações mais coerentes para aplicação da expressão (3.2) relativa 
ao cálculo da distância $e$, além da disposição por quatro cabos oblíquos, são: $\beta \sim 0^{\circ} \mathrm{e}$ $\gamma=90^{\circ}$, onde tem-se $e=\mathrm{d}$ e para $\beta>0^{\circ}$ e $\gamma=90^{\circ}$, onde $e<\mathrm{d}+\mathrm{H}$.

Para as combinações entre $\beta$ e $\gamma$, que resultam em valores significativos da distância $e$, o estudo da instabilidade lateral das vigas não protendidas pode ser definido.

Assim, para a viga apoiada sobre garfos indeformáveis, a condição $\phi(x= \pm a)=0$ de giro nulo nos apoios, deve ser substituída por:

$$
M_{t} \underset{x \rightarrow \pm a}{\mid}=G J_{t} \phi^{\prime} \underset{x= \pm a}{\mid}=-R_{x} \phi \underset{x= \pm a}{\mid}
$$

Na variável m:

$$
G J_{t} \frac{\phi^{\prime}(m)}{a}=-R_{x} \phi(m)
$$

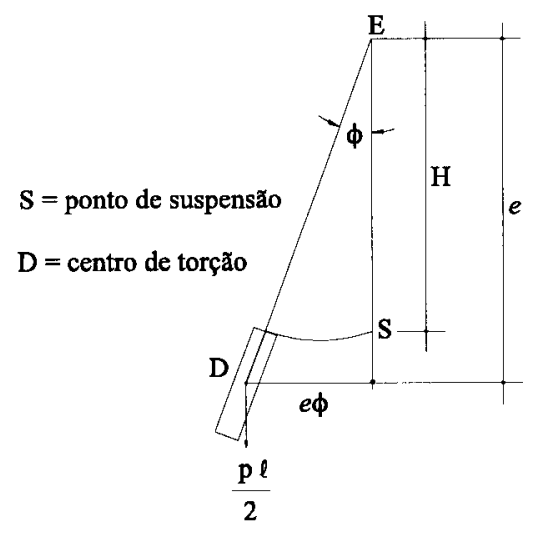

pois,

$$
\begin{gathered}
\phi^{\prime}(x)=\frac{1}{a} \phi^{\prime}(m) \\
\text { para } x=m a \\
\text { Para } \mathrm{m}=1, \text { resulta: } \\
G J_{t} \frac{\phi^{\prime}(1)}{a}=-R_{x} \phi(1)
\end{gathered}
$$

Figura 3.2 - Vista em corte da viga deslocada.

[ CATANIA \& COCCHI (1985)]

Para flambagem em uma única onda e desprezando os termos com potência $\alpha>2$ na expressão de $\phi$, com referência ao estudo da viga apoiada sobre garfos indeformáveis, tem-se as expressões (3.7) e (3.8), onde os coeficientes $A_{0}$ à $A_{12}$ da série foram utilizados:

$$
\begin{aligned}
\phi & =A_{0}\left[1-\alpha\left(\frac{1}{2} m^{2}-\frac{1}{6} m^{4}+\frac{1}{30} m^{6}\right)+\right. \\
& \left.+\alpha^{2}\left(\frac{1}{24} m^{4}-\frac{7}{180} m^{6}+\frac{13}{840} m^{8}-\frac{7}{2700} m^{10}+\frac{1}{3960} m^{12}\right)\right]
\end{aligned}
$$




$$
\begin{aligned}
\phi^{\prime}= & A_{0}\left[-\alpha\left(m-\frac{2}{3} m^{3}+\frac{1}{5} m^{5}\right)+\right. \\
& \left.+\alpha^{2}\left(\frac{1}{6} m^{3}-\frac{7}{30} m^{5}+\frac{13}{105} m^{7}-\frac{7}{270} m^{9}+\frac{1}{330} m^{11}\right)\right]
\end{aligned}
$$

Então, nas extremidades da viga, onde $\mathrm{m}$ é igual a 1 , tem-se:

$$
\begin{gathered}
\phi(1)=A_{0}\left[1-\frac{11}{30} \alpha+\frac{6617}{415800} \alpha^{2}\right] \\
\phi^{\prime}(1)=A_{0}\left[-\frac{8}{15} \alpha+\frac{356}{10395} \alpha^{2}\right]
\end{gathered}
$$

Substituindo as expressões (3.9) e (3.10) na equação (3.6), resulta:

$$
\frac{\ell}{2} \frac{R_{x}}{G J_{t}}=\frac{\frac{8}{15} \alpha-\frac{356}{10395} \alpha^{2}}{1-\frac{11}{30} \alpha+\frac{6617}{415800} \alpha^{2}}=f(\alpha)
$$

A expressão da carga crítica, conforme definida no capítulo 2 para vigas sobre apoios deformáveis, para o valor de $\alpha$ obtido da expressão (3.11), é:

$$
p_{c r i t}=16 \sqrt{\alpha_{c r i t}} \frac{\sqrt{E I_{y} G J_{t}}}{\ell^{3}}
$$

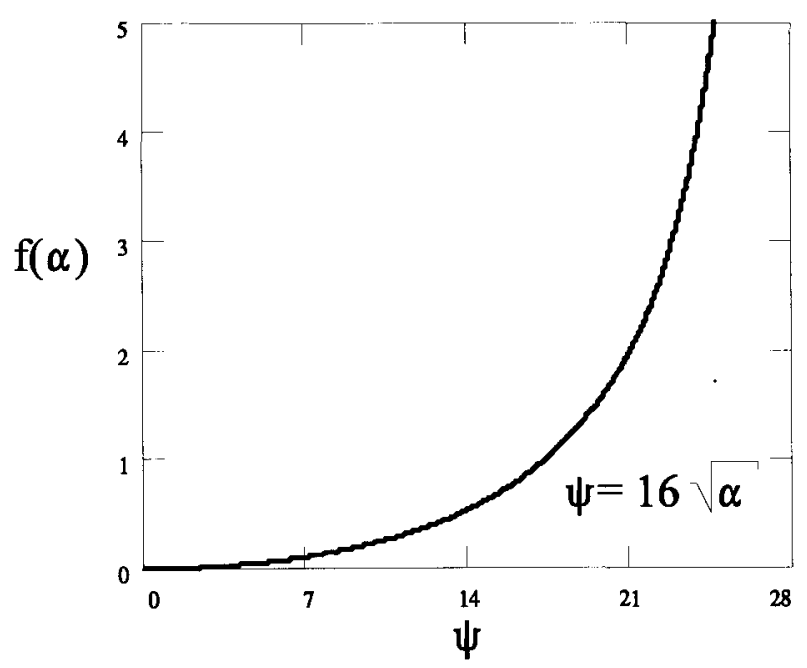

Figura 3.3 - Gráfico da função $f(\alpha)$ em função de $\psi$. 
A curva apresentada na figura 3.3 representa a função $f(\alpha)$ conforme definida na expressão (3.11), onde:

$$
\psi=16 \sqrt{\alpha_{c r i t}}
$$

Para cada valor de $\mathrm{f}(\alpha)$ obtido da expressão (3.11) em função de $\mathrm{R}_{\mathrm{x}}, \mathrm{GJ}_{\mathrm{t}} \mathrm{e} \ell$, temse na curva da figura 3.3 um correspondente valor de $\psi$, que na expressão (3.12), resulta a carga crítica na fase de suspensão.

\subsubsection{CABOS RETOS}

\subsubsection{Suspensão por 2 pontos}

O uso de cabos retos para a suspensão das vigas representa uma disposição mais comum em relação à inclinada, e o posicionamento dos cabos a uma determinada distância a partir das extremidades, aumenta a estabilidade nesta fase, e também o valor da carga crítica. Este estudo é oportuno em alternativa a uma disposição mais genérica do esquema de içamento, como o esquema oblíquo, por ser amplamente empregado.

Para uma viga de seção retangular, suspensa em dois pontos equidistantes do eixo de simetria, conforme a figura 3.4, a expressão do momento fletor no trecho entre os cabos, para $\mathrm{x}=\mathrm{ma}$, e origem dos eixos coordenados na seção de simetria é:

$$
\begin{aligned}
& M(x)=M(m)=p a(\bar{d} a+m a)-p(a+m a) \frac{1}{2}(a+m a) \\
& M(m)=\frac{1}{2} p a^{2}\left(\bar{\gamma}^{2}-m^{2}\right) \quad ; \quad \text { para } \bar{\gamma}^{2}=(2 \bar{d}-1)
\end{aligned}
$$

A equação regente da instabilidade lateral para viga de seção retangular pode ser escrita por:

$$
\phi^{\prime \prime}(x)+\frac{M_{z}^{2}(x)}{E I_{y} G J_{t}} \phi(x)=0
$$

De forma que, para:

$$
\phi^{\prime}(x)=\frac{1}{a} \phi^{\prime}(m) \quad e \quad \phi^{\prime \prime}(x)=\frac{1}{a^{2}} \phi^{\prime \prime}(m)
$$



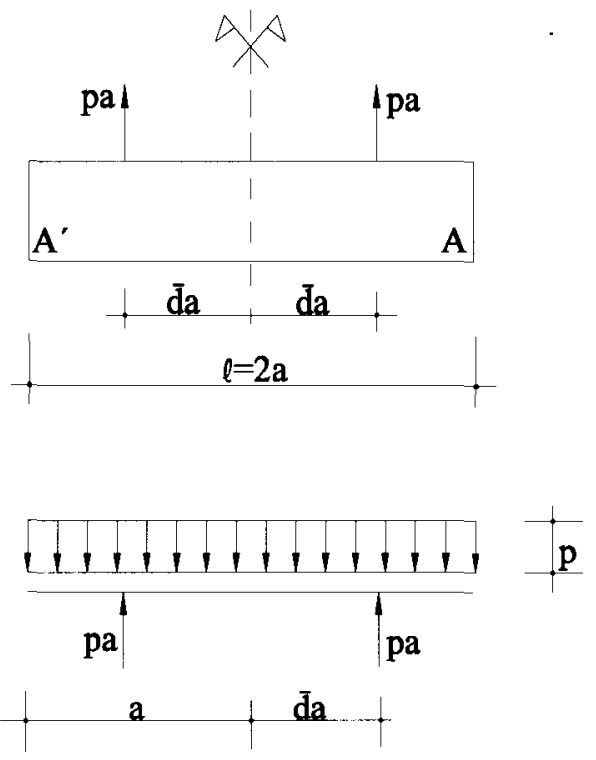

Figura 3.4 - Viga suspensa por cabos retos com balanços. [ CATANIA \& COCCHI (1985)]

e substituindo (3.14) em (3.15), na variável m, tem-se:

$$
\phi^{\prime \prime}(m)+\frac{p^{2} a^{6}}{4 E I_{y} G J_{t}}\left(\bar{\gamma}^{2}-m^{2}\right)^{2} \phi(m)=0
$$

Definindo m como:

$$
m=\bar{\gamma} n
$$

e relacionando as derivadas em relação a n:

$$
\phi^{\prime \prime}(m)=\frac{1}{\bar{\gamma}^{2}} \phi^{\prime \prime}(n)
$$

A expressão diferencial em relação a variável $n$ é:

$$
\phi^{\prime \prime}(n)+\frac{p^{2} a^{6} \bar{\gamma}^{6}}{4 E I_{y} G J_{t}}\left(1-n^{2}\right)^{2} \phi(n)=0
$$

onde pode-se designar $\alpha$ e $\alpha^{\prime}$ por:

$$
\begin{aligned}
& \alpha=\frac{p^{2} a^{6}}{4 E I_{y} G J_{t}} \\
& \alpha^{\prime}=\alpha \bar{\gamma}^{6}
\end{aligned}
$$

Enfim: 


$$
\phi^{\prime \prime}(n)+\alpha^{\prime}\left(1-n^{2}\right)^{2} \phi(n)=0
$$

Pode-se fazer uma analogia entre a equação diferencial (3.22) e a equação (2.7) trocando nesta última $\alpha$ por $\alpha^{\prime}$ e $\mathrm{n}$ por $\mathrm{m}$.

Conforme desenvolvido para a equação (2.7), tem-se idêntica solução para a expressão diferencial (3.22). Assim:

$$
\phi=A_{0}^{\cdot}\left(1+A_{2} \frac{m^{2}}{\bar{\gamma}^{2}}+A_{4} \frac{m^{4}}{\bar{\gamma}^{4}}+\ldots\right)=A_{0}^{*} f(m)
$$

onde os coeficientes podem ser expressos por:

$$
\begin{aligned}
& A_{2}=-\frac{1}{2} \alpha^{\prime}=-\frac{1}{2} \alpha \bar{\gamma}^{6} \\
& A_{4}=\frac{1}{6} \alpha^{\prime}+\frac{1}{24} \alpha^{\prime 2}=\frac{\alpha \bar{\gamma}^{6}}{6}+\frac{\alpha^{2} \bar{\gamma}^{12}}{24} \\
& A_{6}=-\frac{\alpha^{\prime}}{30}-\frac{7 \alpha^{\prime 2}}{180}-\frac{\alpha^{\prime 3}}{720}=-\frac{\alpha \bar{\gamma}^{6}}{30}-\frac{7 \alpha^{2} \bar{\gamma}^{12}}{180}-\frac{\alpha^{3} \gamma^{18}}{720} \\
& A_{8}=\frac{13 \alpha^{\prime 2}}{840}+\frac{11 \alpha^{\prime 3}}{5040}+\frac{\alpha^{4}}{40320}=\frac{13 \alpha^{2} \bar{\gamma}^{12}}{840}+\frac{11 \alpha^{3} \bar{\gamma}^{18}}{5040}+\frac{\alpha^{4} \gamma^{24}}{40320}
\end{aligned}
$$

Para impor a condição de continuidade entre os trechos, através do giro por torção nos apoios, é necessário definir a expressão do ângulo $\phi$ de torção nos balanços.

O momento fletor no balanço, vale:

$$
M(m)=-p(a-m a) \frac{(a-m a)}{2}=-\frac{1}{2} p a^{2}(1-m)^{2}
$$

Chamando de $\phi_{\mathrm{S}}$ o ângulo de torção no trecho do balanço, da expressão do momento fletor $\mathrm{M}=\mathrm{M}_{\mathrm{z}}$ deste trecho, tem-se a expressão diferencial que segue:

$$
\phi_{S}^{\prime \prime}(x)+\frac{M_{z}^{2}(x)}{E I_{y} G J_{t}} \phi_{S}(x)=0
$$

Em função da variável m:

$$
\phi_{S}^{\prime \prime}(m)+\alpha(1-m)^{4} \phi_{S}(m)=0
$$

onde $\alpha$ é o definido anteriormente na expressão (3.21).

Seja a lei geral escolhida para a série de potência: 


$$
\begin{aligned}
\phi_{S}(m)= & \sum_{i=2 j, 1}^{\infty} B_{i}(1-m)^{i}+\sum_{k=2 j}^{\infty} B_{k}(1-m)^{k} \\
\phi_{S}(m)= & B_{0}\left[1+B_{2}(1-m)^{2}+B_{4}(1-m)^{4}+\ldots\right]+ \\
& +B_{1}\left[(1-m)+B_{3}(1-m)^{3}+B_{5}(1-m)^{5}+\ldots\right]
\end{aligned}
$$

onde as constantes com índices pares são definidas por:

$$
\begin{array}{ll}
B_{2}=B_{4}=0 & B_{14}=B_{16}=0 \\
B_{6}=-\frac{1}{30} \alpha & B_{18}=-\frac{1}{1211760} \alpha^{3} \\
B_{8}=B_{10}=0 & B_{20}=B_{22}=0 \\
B_{12}=\frac{1}{3960} \alpha^{2} & B_{24}=\frac{1}{668891520} \alpha^{4}
\end{array}
$$

Para as condições de contorno do trecho em balanço, o momento de torção nas extremidades livres é nulo, o que resulta em $\phi_{\mathrm{S}}{ }^{\prime}(1)=0$. Resolvendo a primeira derivada da função (3.28), obtém-se $B_{1}=0$, restando então, os termos pares da série. Assim, para o trecho do balanço, o ângulo de torção é expresso pela equação:

$$
\phi_{S}=B_{0}\left[1+B_{6}(1-m)^{6}+B_{12}(1-m)^{12}+\ldots\right]=B_{0} g(m)
$$

Nos pontos de fixação dos cabos, para estabelecer as condições de continuidade, deve valer a seguinte igualdade:

$$
\begin{gathered}
\left.\phi\right|_{m=\bar{d}}=\left.\phi_{S}\right|_{m=\bar{d}} \\
A_{0} f(\bar{d})=B_{0} g(\bar{d})
\end{gathered}
$$

Para a igualdade das rotações, tem-se:

$$
G J_{t}\left(\phi_{S}^{\prime}-\phi^{\prime}\right) \underset{x=\bar{d} a}{\mid}=\frac{1}{2} p \operatorname{le} \phi(x) \underset{x=\bar{d} a}{\mid}=p a \text { e } \phi(x) \underset{x=\bar{d} a}{\mid}
$$

e nos pontos de fixação dos cabos, em $\mathrm{m}=\overline{\mathrm{d}}$, resulta:

$$
\frac{G J_{t}}{a}\left(B_{0} g^{\prime}(\bar{d})-A_{0}^{\prime} f^{\prime}(\bar{d})\right)=p a e A_{0}^{\prime} f(\bar{d})=p a e B_{0} g(\bar{d})
$$

Da função $\alpha$, definida na equação (3.21), pode-se extrair o valor de $\mathrm{p}$ : 


$$
p=2 \frac{\sqrt{E I_{y} G J_{t} \alpha}}{a^{3}}
$$

e eliminando $\mathrm{A}_{0}{ }^{*}$ e $\mathrm{B}_{0}$ na equação (3.33), tem-se:

$$
\begin{gathered}
\frac{G J_{t}}{a}\left(B_{0} g^{\prime}(\bar{d})-A_{0}^{\prime} f^{\prime}(\bar{d})\right)=2 \frac{\sqrt{E I_{y} G J_{t} \alpha}}{a^{3}} \text { a e } B_{0} g(\bar{d}) \\
\frac{B_{0} g^{\prime}(\bar{d})-A_{0}^{\prime} f^{\prime}(\bar{d})}{B_{0} g(\bar{d})}=2 \frac{e}{a} \sqrt{\frac{\alpha E I_{y}}{G J_{t}}}
\end{gathered}
$$

Aplicando a equação (3.31) na equação (3.36), resulta:

$$
\therefore \quad 2 \frac{e}{a} \sqrt{\frac{\alpha E I_{y}}{G J_{t}}}=\frac{g^{\prime}(\bar{d})}{g(\bar{d})}-\frac{f^{\prime}(\bar{d})}{f(\bar{d})}
$$

de forma que, a expressão que fornece o valor de $\alpha$ correspondente à instabilidade lateral é:

$$
2 \frac{e}{a} \sqrt{\frac{E I_{y}}{G J_{t}}}=\left[\frac{g^{\prime}(\bar{d})}{g(\bar{d})}-\frac{f^{\prime}(\bar{d})}{f(\bar{d})}\right] \frac{1}{\sqrt{\alpha}}=j(\alpha, \bar{d})
$$

A expressão $f(\alpha, \bar{d})$ pode ser escrita por:

$$
\begin{gathered}
f(\alpha, \bar{d})=A(\alpha, \bar{d})-B(\alpha, \bar{d})+C(\alpha, \bar{d}) \\
A(\alpha, \bar{d})=1-\frac{1}{2} \alpha(2 \bar{d}-1)^{2}+\left[\frac{\alpha}{6}+\frac{\alpha^{2}}{24}(2 \bar{d}-1)^{3}\right](2 \bar{d}-1)^{4} \bar{d}^{4} \\
B(\alpha, \bar{d})=\left[\frac{\alpha}{30}+\frac{7 \alpha^{2}}{180}(2 \bar{d}-1)^{3}+\frac{\alpha^{3}}{720}(2 \bar{d}-1)^{6}\right] \bar{d}^{6} \\
C(\alpha, \bar{d})=\left[\frac{13 \alpha^{2}}{840}(2 \bar{d}-1)^{2}+\frac{11 \alpha^{3}}{5040}(2 \bar{d}-1)^{5}+\frac{\alpha^{4}}{40320}(2 \bar{d}-1)^{8}\right] \bar{d}^{8}
\end{gathered}
$$

e a expressão $g(\alpha, \overline{\mathrm{d}})$ : 


$$
\begin{aligned}
g(\alpha, \bar{d})= & 1-\frac{\alpha}{30}(1-\bar{d})^{6}+\frac{\alpha^{2}}{3960}(1-\bar{d})^{12}-\frac{\alpha^{3}}{1211760}(1-\bar{d})^{18}+ \\
& +\frac{\alpha^{4}}{668891520}(1-\bar{d})^{24}
\end{aligned}
$$

Para as derivadas $\mathrm{f}^{\prime}(\alpha, \overline{\mathrm{d}})$ e $\mathrm{g}^{\prime}(\alpha, \overline{\mathrm{d}})$, e para um valor pré-definido de $\overline{\mathrm{d}}$, define-se a função $\mathrm{j}(\alpha)$.

Os gráficos ilustrados nas figuras 3.5 à 3.8, para diferentes valores de $\overline{\mathrm{d}}$, representam as curvas da função $\mathrm{j}(\alpha)$, para os valores de $\alpha$ que resultam na carga crítica.

$$
p_{\text {crit }}=16 \sqrt{\alpha_{c r i t}} \frac{\sqrt{E I_{y} G J_{t}}}{\ell^{3}}
$$

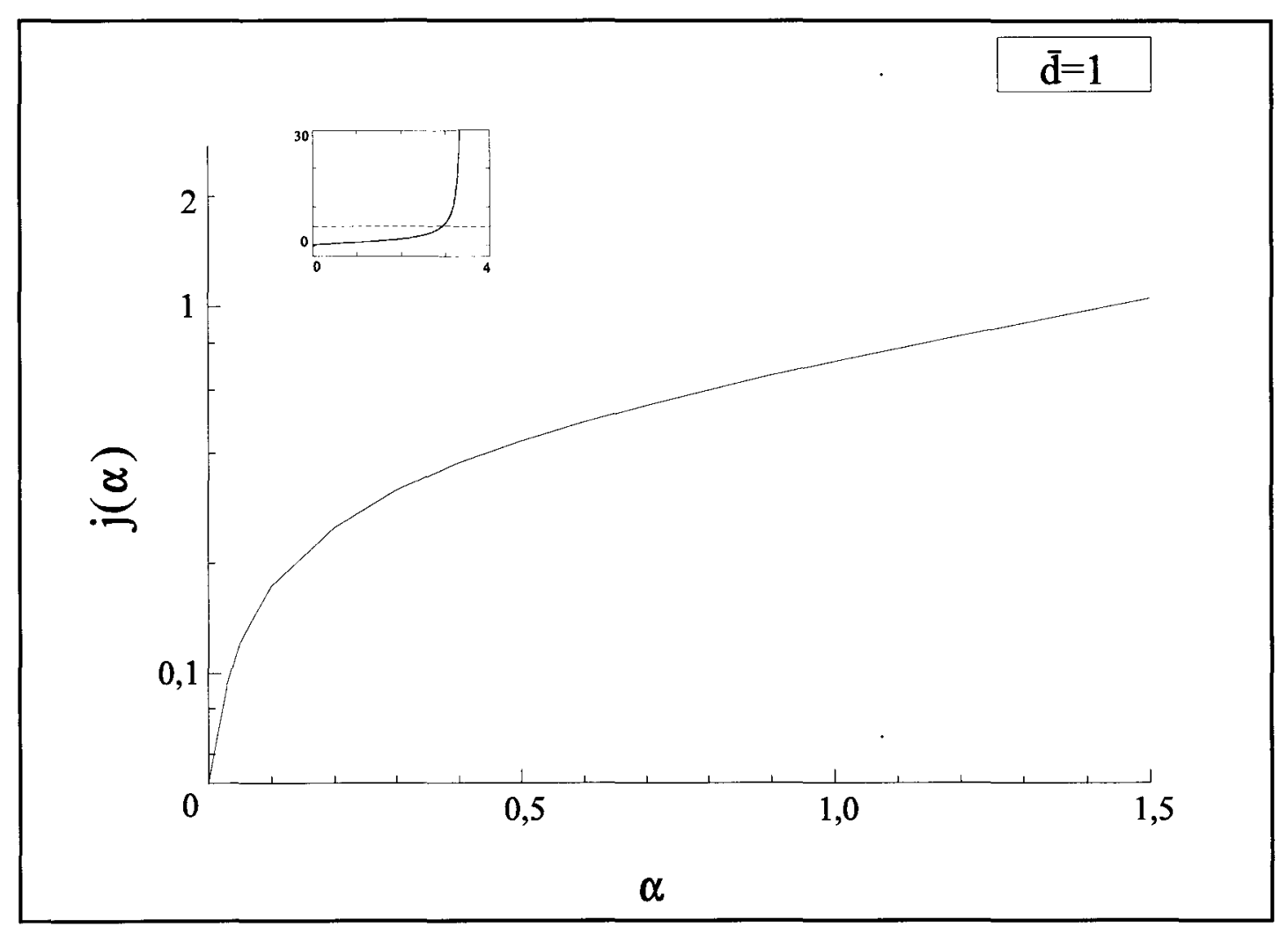

Figura 3.5 - Gráfico da função $\mathrm{j}(\alpha)$ para $\mathrm{d}=1$. 
$\overline{\mathrm{d}}=0$
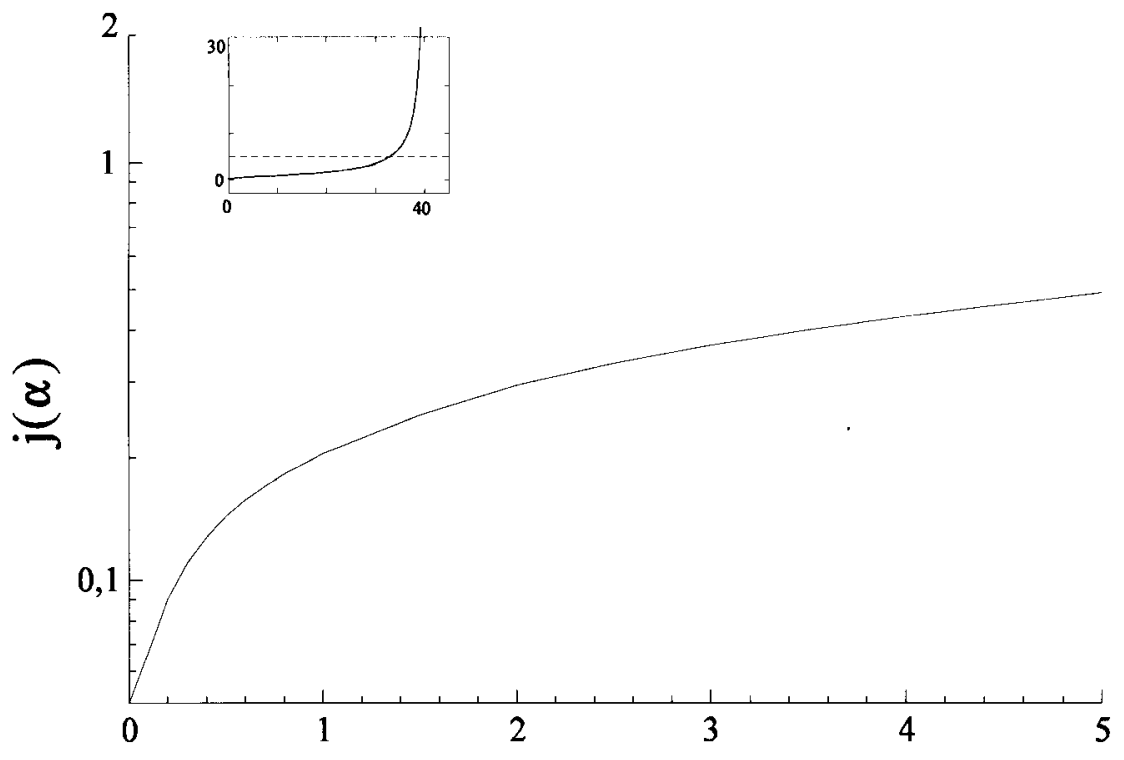

$\alpha$

Figura 3.6 - Gráfico da função $\mathrm{j}(\alpha)$ para $\mathrm{d}=0$.

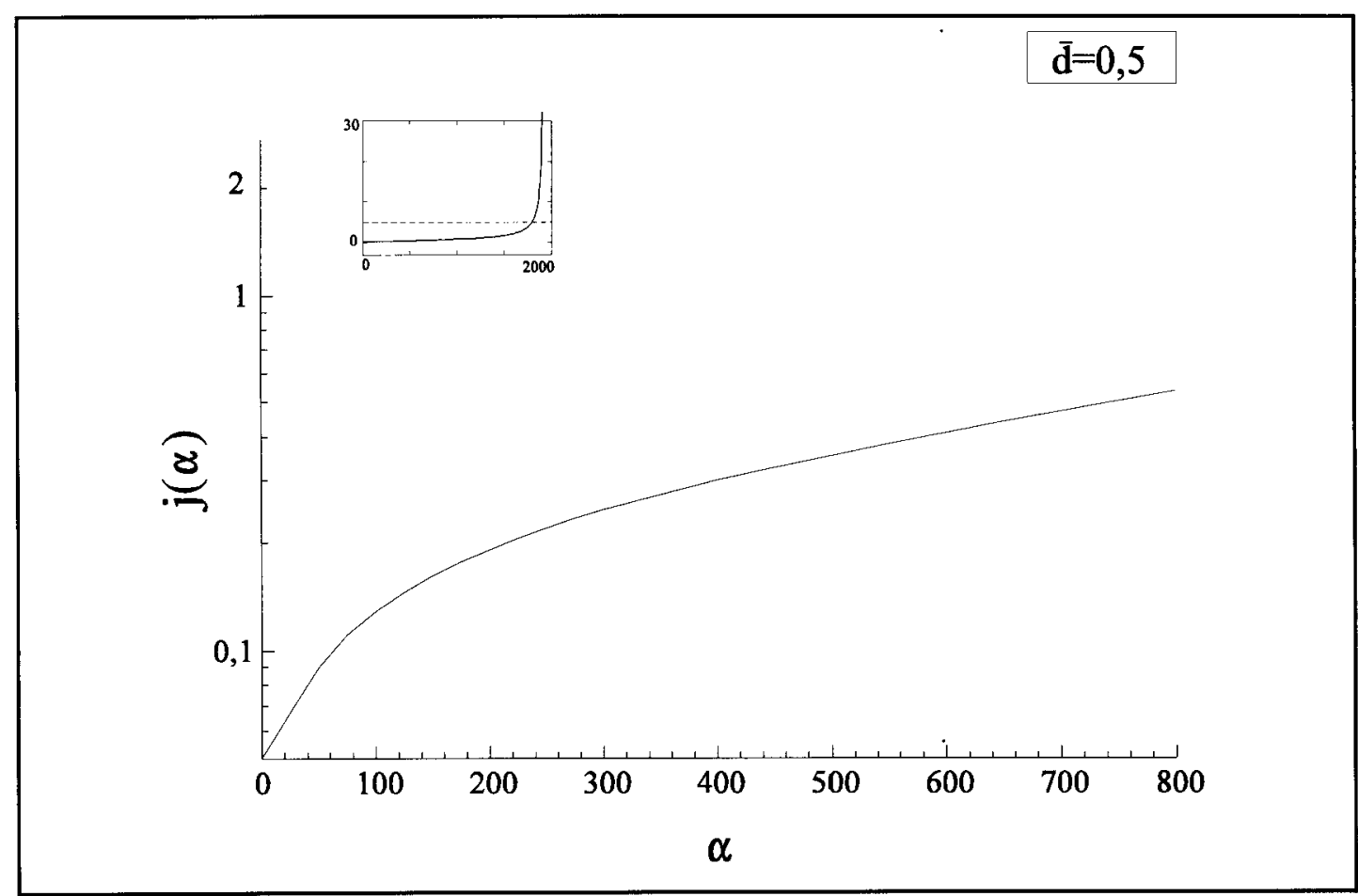

Figura 3.7 - Gráfico da função $j(\alpha)$ para $\bar{d}=0,5$. 


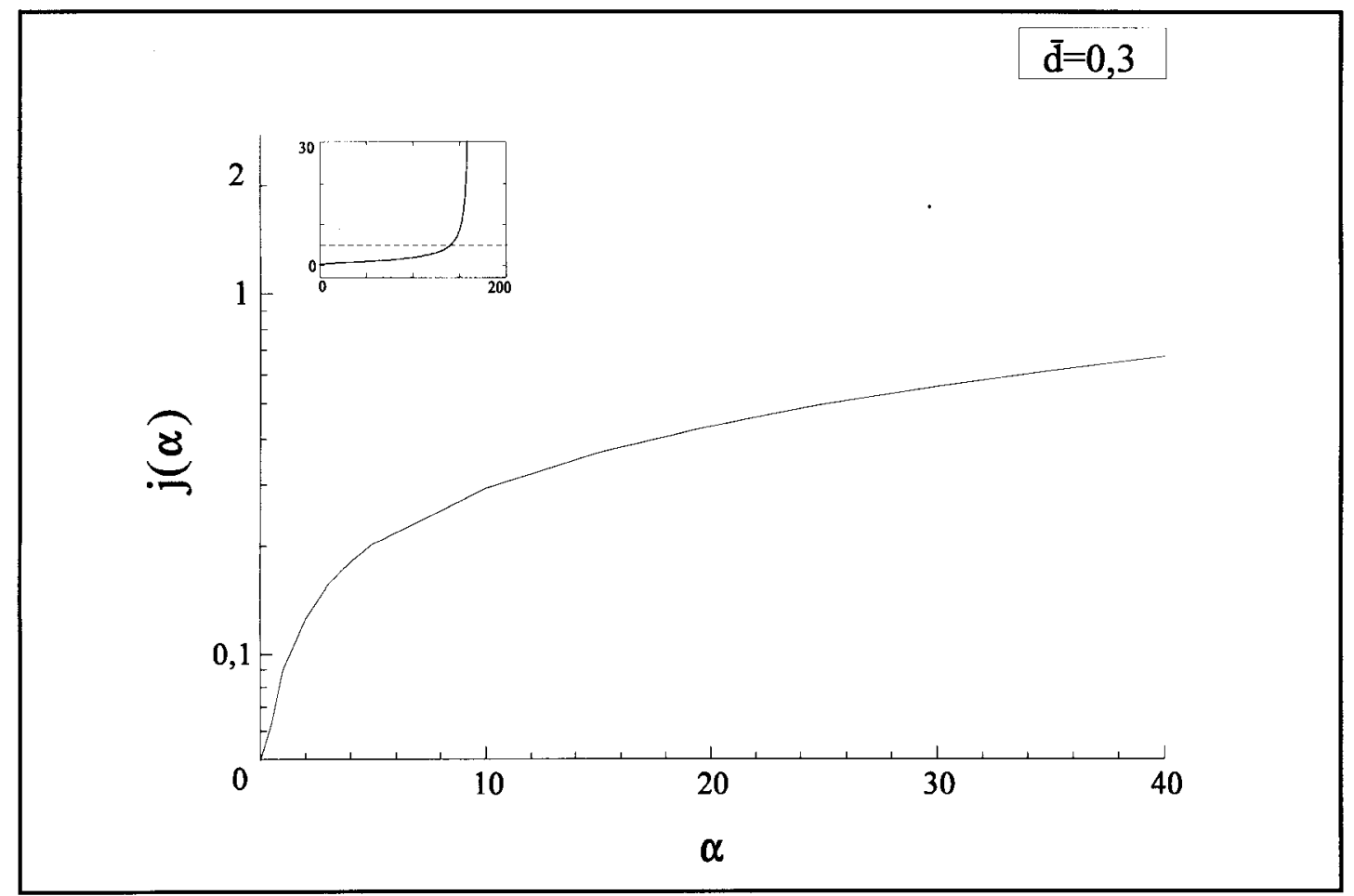

Figura 3.8 - Gráfico da função $\mathrm{j}(\alpha)$ para $\mathrm{d}=0,3$.

A variação de $\overline{\mathrm{d}}$ com $\psi$ está representada na figura 3.9 , para valores de $\mathrm{j}(\alpha, \overline{\mathrm{d}})$ tendendo a infinito. Na curva de $\bar{d}=1$ para $j(\alpha, \bar{d}) \sim \infty$ resulta $\psi=28,3$; da mesma forma, para $\overline{\mathrm{d}}=0,8$ tem-se $\psi=59,65$ quando a função $\mathrm{j}(\alpha, \overline{\mathrm{d}}) \sim \infty$, e assim para as demais curvas. Para valores tendendo a infinito da função $\mathrm{j}$, os apoios representam ligações rígidas à torção.

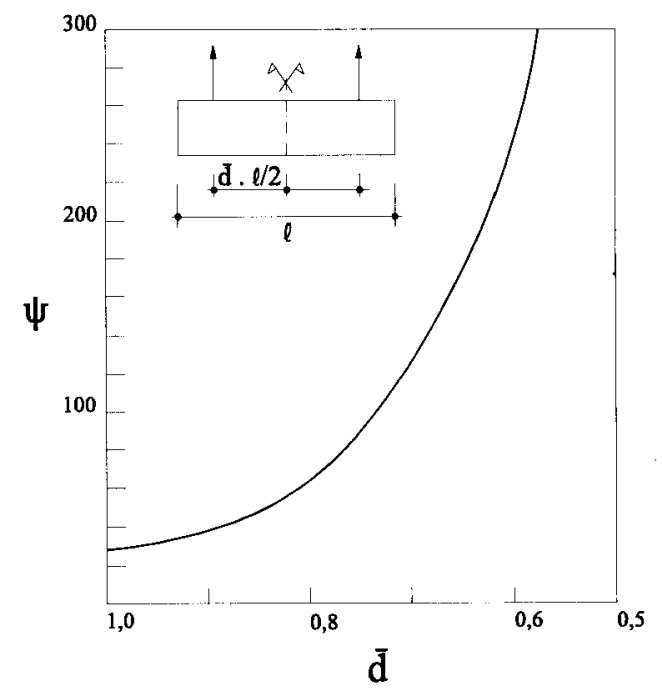

Figura 3.9 - Gráfico de $\psi$ em função de đ. [ CATANIA \& COCCHI (1985) ] 
Conforme explícito no gráfico da figura 3.9, a presença dos balanços aumenta sensivelmente o valor da carga crítica. Assim, um modo efîcaz de aumentar o fator de segurança é reduzir a distância entre os pontos de içamento.

Entretanto, o posicionamento dos balanços deve ser definido de forma a não gerar instabilidade nos trechos em balanço. Será tratado neste estudo, o caso de uma viga suspensa na seção central. A disposição dos balanços nos quartos de vão fornece ótimos resultados. Porém a localização definitiva dos pontos de içamento deve ser subordinada a um efetivo controle das tensões em todas as seções críticas.

Em relação à suspensão com balanços, CATANIA \& COCCHI (1985) apresentam a expressão da distância crítica d, que conforme mostrado na figura 3.1, pode ser definida como a distância do ponto teórico de suspensão ao CG da viga.

Assim,

$$
d_{c r i t}=k\left(1-\alpha k_{1}\right) \frac{p \ell^{4}}{E I_{y}}
$$

onde:

$$
\begin{aligned}
& k(\bar{d})=\frac{3(1-\bar{d})^{5}+m}{60} \\
& k_{1}(\bar{d})=\frac{3(1-\bar{d})^{11}+m n}{1800 k}
\end{aligned}
$$

sendo $\mathrm{m}$ e $\mathrm{n}$ definidos por:

$$
\begin{aligned}
& n=15 \bar{\gamma} \bar{d}^{2}-5 \bar{\gamma} \bar{d}^{4}+\bar{d}^{4}+\bar{d}^{6} \\
& m=15 \bar{\gamma}^{4} \bar{d}-10 \bar{\gamma}^{2} \bar{d}^{3}+3 \bar{d}^{5}
\end{aligned}
$$

Os parâmetros k e $k_{1}$ são funções de d (distância da seção de simetria ao ponto de suspensão), e seus valores encontram-se dispostos na tabela 3.1.

Para $\alpha=0$, tem-se:

$$
d_{c r i t}=\frac{k p \ell^{4}}{E I_{y}}
$$

A expressão de $\mathrm{k}(\overline{\mathrm{d}})$ pode ser desenvolvida substituindo $m$ da equação (3.43) e $\bar{\gamma}$ da expressão (3.14): 


$$
k=\frac{1}{60}\left[3-30 \bar{d}^{2}+40 \bar{d}^{3}-5 \bar{d}^{4}\right]
$$

Tabela 3.1 - Valores dos parâmetros k em função de d.

\begin{tabular}{|c|c|c|c||}
\hline $\bar{d}$ & $k(\bar{d})$ & $\frac{1}{k(\bar{d})}$ & $k_{1}(\bar{d})$ \\
\hline 0 & & 20,00 & 0,0333 \\
\hline 0,1 & 0,0500 & 21,90 & 0,0126 \\
\hline 0,2 & 0,0351 & 28,41 & 0,0080 \\
\hline 0,3 & 0,0223 & 44,79 & 0,0063 \\
\hline 0,4 & 0,0105 & 94,94 & 0,0032 \\
\hline 0,5 & 0,0031 & 320,00 & 0,0005 \\
\hline 0,6 & 0,0032 & 312,50 & 0,0037 \\
\hline 0,7 & 0,0137 & 73,22 & 0,0269 \\
\hline 0,8 & 0,0372 & 26,88 & 0,0829 \\
\hline 0,9 & 0,0763 & 13,10 & 0,1894 \\
\hline 1 & 0,1333 & 7,50 & 0,3333 \\
\hline
\end{tabular}

Supor $\alpha=0$ significa assumir uma rigidez infinita à torção, porque entende-se $\mathrm{EI}_{\mathrm{y}}$ como um fator finito, já que $\alpha$ é função de $\mathrm{EI}_{\mathrm{y}}$ e $\mathrm{GJ}_{\mathfrak{t}}$ e inversamente proporcional à estas constantes.

A figura 3.10 representa um diagrama com os valores de $\mathrm{k} \mathrm{e} 1 / \mathrm{k}$.

Da expressão (3.42), pode-se isolar $p$, de forma que para $d_{\text {crit }}$ tenha-se $p_{\text {crit }}$

$$
p_{c r i t}=\frac{E I_{y} d}{\ell^{4}} \frac{1}{k\left(1-\alpha k_{1}\right)}
$$

Por aproximação, assumindo $\alpha=0$ :

$$
\begin{aligned}
& \bar{d}=0 \quad » \quad \frac{1}{k}=20 \quad » \quad \therefore p_{\text {crt }}=20 E I_{y} \frac{d}{\ell^{4}} \\
& \bar{d}=1 \quad \gg \quad \frac{1}{k}=7,5 \quad » \quad \therefore p_{\text {crut }}=7,5 E I_{y} \frac{d}{l^{4}}
\end{aligned}
$$




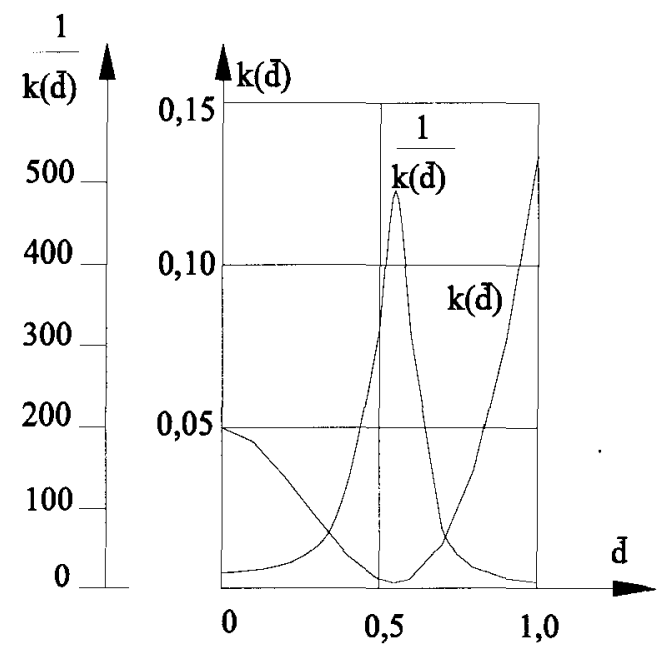

Figura 3.10 - Diagrama dos valores de $\mathbf{k}$ e $\mathbf{1} / \mathbf{k}$.

[ CATANIA \& COCCHI (1985)]

Os valores máximos de $\mathrm{k}(\overline{\mathrm{d}})$ ocorrem para:

$$
\begin{aligned}
\frac{d k(\bar{d})}{d(\bar{d})} & =-\frac{2 * 30}{60} \bar{d}+\frac{3 * 40}{60} \bar{d}^{2}-\frac{4 * 5}{60} \bar{d}^{3}= \\
& =-\bar{d}+2 \bar{d}^{2}-\frac{1}{3} \bar{d}^{3}=0
\end{aligned}
$$

que resulta em:

$$
\bar{d}_{m i x}=0,55
$$

Para $\overline{\mathrm{d}}=0,55$ o valor de $\mathrm{p}_{\text {crit }}$ pode ser expresso:

$$
p_{\text {crit }}=\frac{E I_{y} d}{\ell^{4}} \frac{1}{k} \quad \rightarrow \quad \text { para } \alpha=0
$$

onde k resulta em:

$$
k=\frac{1}{60}\left(3-30 * 0,55^{2}+40 * 0,55^{3}-5 * 0,55^{4}\right)
$$

A expressão da carga crítica é, então:

$$
p_{\text {crit }}=489,9 \frac{E I_{y}}{\ell^{4}} d
$$

Assim, para $\overline{\mathrm{d}}=0,55$ o valor da carga crítica é cerca de 65 vezes maior que para $\bar{d}=1$, ou seja, para cabos presos nas extremidades, representado na equação (3.48). 


\subsubsection{Suspensão por 3 pontos}

A suspensão por 3 cabos verticais pode ser adotada para aumentar a segurança da suspensão, entretanto, introduz esforços de tração na seção de simetria, os quais devem ser verificados.

Na suspensão por 3 pontos, representada no gráfico da figura 3.11, CATANIA \& COCCHI (1985) propõem a expressão (3.54) para o cálculo da carga crítica:

$$
p_{c r i t}=m \frac{\sqrt{E I_{y} G J}}{\ell^{3}}
$$

onde $\mathrm{m}=\mathrm{f}(\mathrm{Q} / \mathrm{p} \ell)$ e $\mathrm{Q}$ é a carga concentrada aplicada com sentido contrário ao peso próprio.

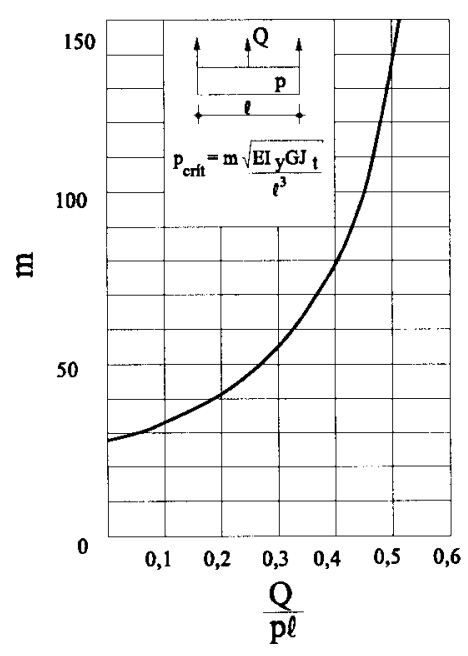

Figura 3.11 - Viga suspensa em 3 pontos.

[ CATANIA \& COCCHI (1985)]

Em uma viga suspensa nas extremidades, submetida a uma carga aplicada na simetria em sentido oposto ao do carregamento relativo ao peso-próprio, para o aumento da carga $Q$, a carga crítica também aumenta.

\subsubsection{Suspensão por 1 ponto}

Para a viga suspensa na seção central de simetria $x=\ell / 2$, como está ilustrado na figura 3.12, CATANIA \& COCCHI (1985) definem a seguinte expressão para o cálculo 
da carga crítica:

$$
p_{\text {crtt }}=20 \frac{E I_{y}}{\ell^{4}} d
$$

que é a mesma equação anteriormente apresentada como (3.48), para $\overline{\mathrm{d}}=0$.

$\mathrm{Na}$ suspensão com uma barra rígida fixa na seção central da viga, tem-se a expressão abaixo:

$$
p_{\text {crit }}=m \frac{\sqrt{E I_{y} G J}}{\ell^{3}} \sqrt{1+\left(\frac{\pi}{K \ell}\right)^{2}}
$$

onde os valores de $\mathrm{m}$ são função de $\mathrm{K} \ell$ para os diversos valores de $\omega$, sendo que $\mathrm{K}$ e $\omega$ são definidos pelas expressões (3.57) e (3.58). No gráfico da figura 3.13 obtém-se o valor de $\mathrm{m}$.

$$
\begin{gathered}
K=\sqrt{\frac{G J_{t}}{E I_{y}} \frac{h^{2}}{4}}, h=\text { altura da viga } \\
\omega=\frac{d}{\ell} \sqrt{\frac{E I_{y}}{G J_{t}}} \frac{1}{\sqrt{1+\frac{\pi^{2}}{(K \ell)^{2}}}}
\end{gathered}
$$
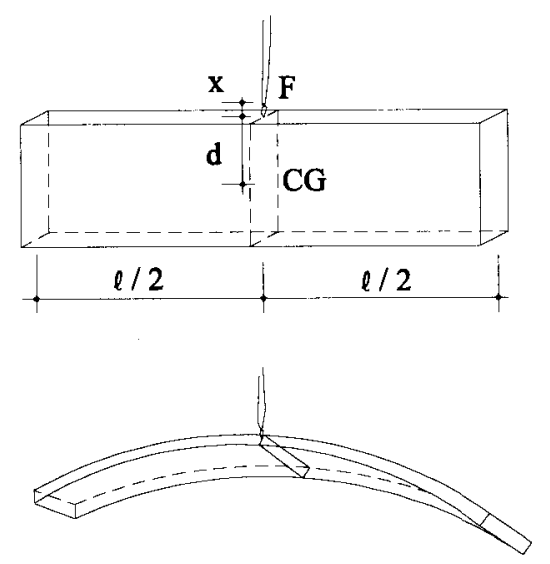

Figura 3.12 - Esquema de viga suspensa no vão central.

CATANIA \& COCCHI (1985) 
Um comentário a ser feito na suspensão única por um ponto na seção central de simetria é que sua apresentação neste texto tem carácter informativo, visto que deve ser realizada em situação especial. A fase transitória deve ser, preferencialmente, compatível à situação em serviço, ou seja, não deve introduzir esforços totalmente opostos.

Por exemplo, para uma viga comum, que irá trabalhar sob esforços de tração na face inferior e de compressão na face superior, não é favorável proceder a suspensão em único ponto no vão central. Os esforços, apesar de serem numericamente equivalentes, são exatamente opostos, como mostra a figura 3.14 .

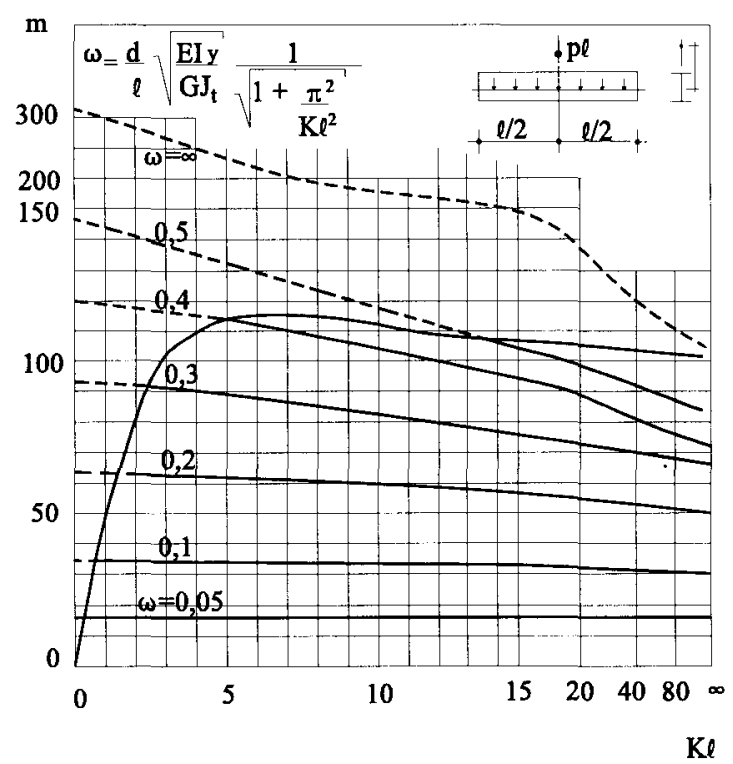

Figura 3.13 - Gráfico de suspensão em x=̨/2. CATANIA \& COCCHI (1985).

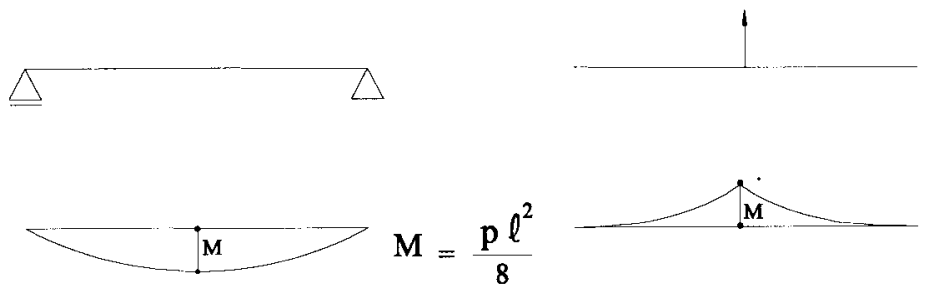




\subsection{VIGAS PROTENDIDAS DURANTE A FASE TRANSITÓRIA DE SUSPENSÃO}

A análise da instabilidade em vigas protendidas de seção duplo $\mathrm{T}$ genérica, de altura constante e cabos de protensão retilíneos, será descrita pelo método da energia potencial, conforme desenvolvido por CATANIA \& COCCHI (1985).

Para o esquema estático da protensão ilustrado na figura (3.15), será considerado em primeira etapa, os efeitos da protensão como ação isolada. Os efeitos da ação conjunta do carregamento aplicado em presença da protensão, compõem a segunda etapa.

A fase de suspensão, analisada pelos autores acima, foi desenvolvida para uma viga vinculada por ligação tipo garfo nas extremidades, rígidos à torção, onde prendemse os cabos de suspensão, em pontos na face superior do apoio. Desta forma, deve-se apenas acrescentar ao estudo da fase de serviço, a contribuição da componente horizontal da força de suspensão do cabo inclinado.

\subsubsection{AÇÃO ISOLADA DA PROTENSÃO}

A energia de deformação, para a viga esquematizada na figura 3.15 é a mesma descrita no item 2.4.2, bem como a energia potencial dos esforços externos. Para a configuração de equilíbrio ilustrada na figura 3.15, o cálculo do trabalho de segunda ordem das componentes de tensão será apresentado a seguir.

Do mesmo modo como foi definido no estudo da fase de serviço, a configuração deformada baseia-se nos deslocamentos $\delta$ do centro de gravidade e nos deslocamentos angulares $\phi$.

Sendo $\mathrm{f}_{0}(\mathrm{x})$ as forças radiais representadas na figura 2.4, para o equilíbrio do cabo de protensão, tem-se:

$$
f_{0}(x)=\frac{N_{P}}{r} \quad \text { e } \quad \frac{1}{r}=\frac{d^{2} w}{d x^{2}}
$$

Conforme a deformada da viga, em um ponto genérico sob o cabo de protensão, 
o deslocamento $\mathrm{w}$ em $\mathrm{z}$ pode ser descrito por: $\mathrm{w}=\delta+\mathrm{e}_{0} \phi$. Assim, em $\mathrm{f}_{0}(\mathrm{x})$ resulta:

$$
f_{0}(x)=N_{P} \frac{d^{2}\left(\delta+e_{0} \phi\right)}{d x^{2}}
$$

À expressão da tensão normal da viga protendida suspensa por cabos, acrescenta-se a parcela do momento fletor $M_{c}$ devido à componente normal da força de suspensão do cabo inclinado. Assim, as tensões normais e cișalhantes da viga podem ser escrita por:

$$
\begin{gathered}
\sigma_{x} \cong-\frac{N_{P}}{A}-\frac{N_{P} e_{0}}{I_{z}} y+\frac{M_{c}}{I_{z}} y \\
\tau_{x y}=0
\end{gathered}
$$

onde o momento $M_{c}$ é definido por: $M_{c}=F_{0} \cdot z_{1}$.
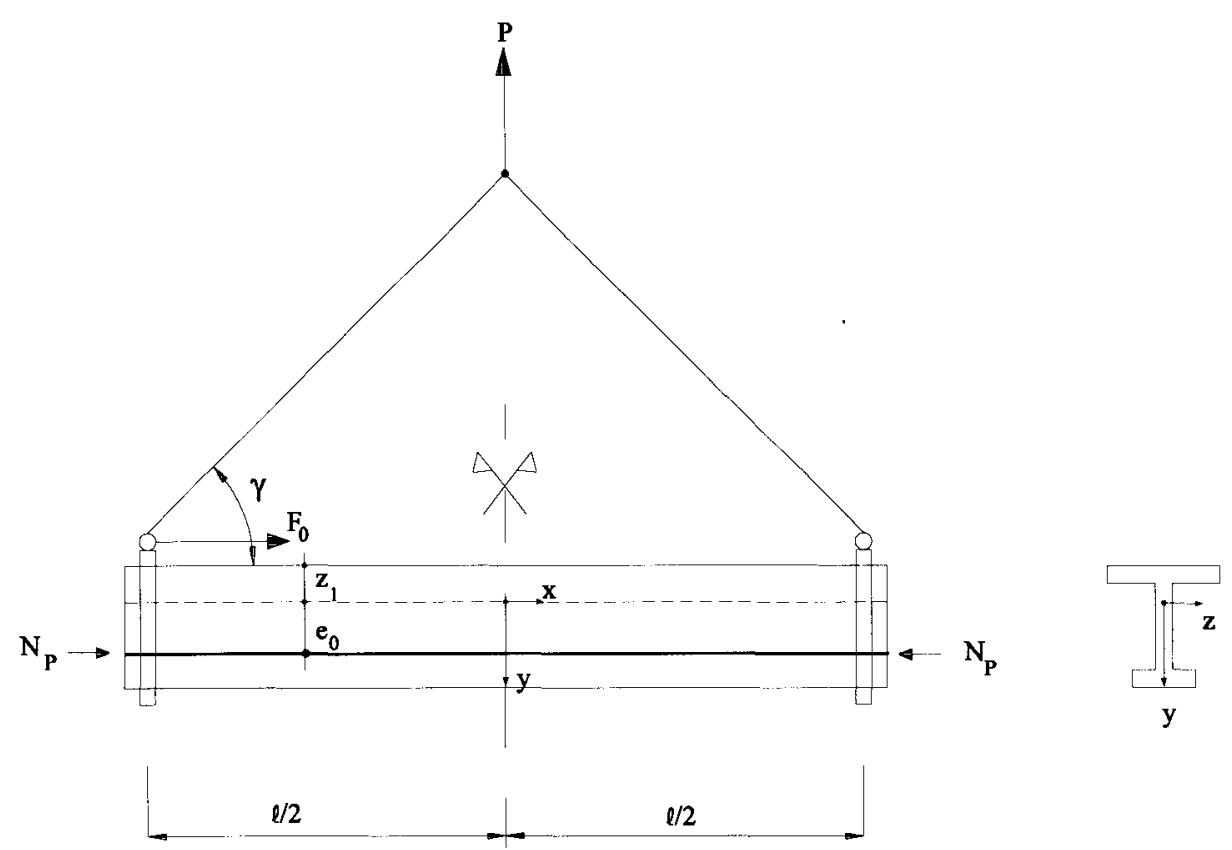

Figura 3.15 - Viga protendida em suspensão. [ CATANIA \& COCCHI (1985) ]

A expressão da energia potencial $T_{2}$ dos esforços internos de $1^{a}$ ordem nas deformações de $2^{\mathrm{a}}$ ordem, como definida anteriormente, é: 


$$
T_{2}=\int_{V} \sigma_{x} \varepsilon_{x}^{(2)} d V
$$

onde $\varepsilon_{\mathrm{x}}{ }^{(2)}$ é deformação de $2^{\mathrm{a}}$ ordem:

$$
\begin{aligned}
& \varepsilon_{x}^{(2)}=\frac{1}{2}\left[\left(\frac{\partial v}{\partial x}\right)^{2}+\left(\frac{\partial w}{\partial x}\right)^{2}\right]=\frac{1}{2}\left[\frac{d}{d x}(\delta+y \phi)\right]^{2} \\
& \varepsilon_{x}^{(2)}=\frac{1}{2}\left[\left(\frac{d \delta}{d x}\right)^{2}+2 y \frac{d \delta}{d x} \frac{d \phi}{d x}+y^{2}\left(\frac{d \phi}{d x}\right)^{2}\right]
\end{aligned}
$$

Desta forma, a expressão da energia potencial dos esforços internos de $2^{\mathrm{a}}$ ordem nas deformações de $1^{\mathrm{a}}$ ordem $\mathrm{T}_{2}$, pode ser escrita como segue:

$$
T_{2}=\int_{V}\left[\left(-\frac{N_{p}}{A}-\frac{N_{p} e_{0}}{I_{z}} y\right) \varepsilon_{x}^{(2)}+\frac{M_{c}}{I_{z}} y \varepsilon_{x}^{(2)}\right] d V
$$

Assim, considerando as contribuições da força normal $\mathrm{N}_{P}$ de protensão e do momento fletor $\mathrm{M}_{\mathrm{c}}$, tem-se:

$$
T_{2}=\left(T_{2}\right)_{N_{P}}+\left(T_{2}\right)_{M_{c}}
$$

O primeiro termo, referente à parcela de contribuição da força normal, calculado no item 2.4.2, é:

$$
\begin{aligned}
& \left(T_{2}\right)_{N_{P}}=\int_{0}^{d / 2} \int_{A}-N_{P}\left(\frac{1}{A}+\frac{e_{0}}{I_{z}} y\right) \cdot\left[\left(\frac{d \delta}{d x}\right)^{2}+2 y \frac{d \delta}{d x} \frac{d \phi}{d x}+y^{2}\left(\frac{d \phi}{d x}\right)^{2}\right] d A d x \\
& \left(T_{2}\right)_{N_{P}}=-N_{P} \int_{0}^{\ell / 2}\left[\left(\frac{d \delta(x)}{d x}\right)^{2}+i_{z}^{2}\left(\frac{d \phi(x)}{d x}\right)^{2}-2 e_{0} \phi \frac{d^{2} \delta(x)}{d x^{2}}\right] d x
\end{aligned}
$$

e a contribuição do momento $M_{c}$ :

$$
\left(T_{2}\right)_{M_{c}}=\int_{V} \frac{M_{c}}{I_{z}} y \varepsilon_{x}^{(2)} d V
$$




$$
\left(T_{2}\right)_{M_{c}}=\frac{1}{2} \int_{V} \frac{M_{c}}{I_{z}} y\left[\left(\frac{d \delta}{d x}\right)^{2}+2 y \frac{d \delta}{d x} \frac{d \phi}{d x}+y^{2}\left(\frac{d \phi}{d x}\right)^{2}\right] d V
$$

Assumindo as igualdades da equação (2.52):

$$
\begin{aligned}
& \left(T_{2}\right)_{M_{c}}=\frac{M_{c}}{I_{z}} \int_{0}^{1 / 2} 2 I_{z} \frac{d \delta}{d x} \frac{d \phi}{d x} d x \\
& \left(T_{2}\right)_{M_{c}}=2 M_{c} \int_{0}^{1 / 2} \frac{d \delta}{d x} \frac{d \phi}{d x} d x
\end{aligned}
$$

Substituindo as expressões das elásticas, para as condições de vinculações assumidas, resulta:

$$
\begin{gathered}
\left(T_{2}\right)_{M_{c}}=2 M_{c} a b \frac{\pi^{2}}{\ell^{2}} \int_{0}^{\ell / 2} \operatorname{sen}^{2} \frac{\pi x}{\ell} d x \\
\left(T_{2}\right)_{M_{c}}=\frac{1}{2} M_{c} a b \frac{\pi^{2}}{\ell}
\end{gathered}
$$

Assim, a expressão final da energia potencial total é:

$$
E P T=E I_{y} \frac{\pi^{4}}{4 \ell^{3}} a^{2}+G J t \frac{\pi^{2}}{4 \ell} b^{2}+N_{P} \frac{\pi^{2}}{4 \ell}\left(e_{0}^{2}-i_{z}^{2}\right) b^{2}+M_{c} a b \frac{\pi^{2}}{2 \ell}
$$

Para a determinação da carga crítica, devem ser simultaneamente satisfeitas as condições determinadas em (2.77), ou seja, as derivadas nulas da EPT em relação aos parâmetros da deformada, consiste em uma configuração de equilíbrio.

$$
\begin{aligned}
& \frac{\partial E P T}{\partial a}=2 E I_{y} \frac{\pi^{4}}{4 l^{3}} a-M_{c} \frac{\pi^{2}}{2 l} b=0 \\
& \frac{\partial E P T}{\partial b}=2 G J_{t} \frac{\pi^{2}}{4 l} b+2 N_{P} \frac{\pi^{2}}{4 l}\left(e_{0}^{2}-i_{z}^{2}\right) b-M_{c} \frac{\pi^{2}}{2 \ell} a=0
\end{aligned}
$$

No limite de instabilidade lateral, anula-se o determinante para que a solução não seja trivial:

$$
\left|\begin{array}{cc}
E I_{y} \frac{\pi^{4}}{2 \ell^{3}} & M_{c} \frac{\pi^{2}}{2 \ell} \\
M_{c} \frac{\pi^{2}}{2 \ell} & \frac{\pi^{2}}{2 \ell}\left[G J_{t}+N_{p}\left(e_{0}^{2}-i_{z}^{2}\right)\right]
\end{array}\right|=0
$$


Resolvendo o determinante, tem-se:

$$
E I_{y} \frac{\pi^{6}}{4 \ell^{4}}\left[G J_{t}+N_{P}\left(e_{0}^{2}-i_{z}^{2}\right)\right]-M_{c}^{2} \frac{\pi^{4}}{4 \ell^{2}}=0
$$

Para as análises da instabilidade lateral, o momento $M_{c}$ é igual a:

$$
M_{c \text { crit }}^{2}=E I_{y} G J_{t} \frac{\pi^{2}}{\ell^{2}}+E I_{y} \frac{\pi^{2}}{\ell^{2}} N_{P}\left(e_{0}^{2}-i_{z}^{2}\right)
$$

\subsubsection{AÇÃO DE EVENTUAIS OUTROS CARREGAMENTOS DISTRIBUÍDOS}

Para a contribuição do carregamento externo definido em série de Fourier, apresentado no desenvolvimento da fase de serviço, item 2.4 .3 , pode-se escrever as derivadas parciais da energia potencial em relação aos parâmetros da deformada:

$$
\begin{aligned}
& \frac{\partial E P T}{\partial a}=2 E I_{y} \frac{\pi^{4}}{4 \ell^{3}} a+F_{0} z_{1} \frac{\pi^{2}}{2 \ell} b=0 \\
& \frac{\partial E P T}{\partial b}=2 G J_{t} \frac{\pi^{2}}{4 \ell} b+2 N_{P} \frac{\pi^{2}}{4 l}\left(e_{0}^{2}-i_{z}^{2}\right) b+F_{0} z_{1} \frac{\pi^{2}}{2 \ell} a+ \\
& -2 d \cdot p \cdot \frac{\ell}{\pi} b=0
\end{aligned}
$$

onde o momento fletor $M_{c}$ foi substituído por $F_{0} \cdot z_{1}$.

O determinante deve ser nulo para que a solução não seja trivial. Assim:

$$
\left|\begin{array}{cc}
E I_{y} \frac{\pi^{4}}{2 \ell^{3}} & F_{0} z_{1} \frac{\pi^{2}}{2 \ell} \\
F_{0} z_{1} \frac{\pi^{2}}{2 \ell} & \frac{\pi^{2}}{2 \ell}\left[G J_{t}+N_{p}\left(e_{0}^{2}-i_{z}^{2}\right)\right]-2 d \cdot p \cdot \frac{\ell}{\pi}
\end{array}\right|=0
$$

Desta forma, admitindo a relação entre $\mathrm{F}_{0}$ e $\mathrm{P}$ :

$$
F_{0}=\frac{P}{2 \operatorname{tg} \gamma}=\frac{p \cdot \ell}{2 \operatorname{tg} \gamma}
$$

Resolvendo o determinante para a relação (3.81): 


$$
E I_{y} G J_{t} \frac{\pi^{6}}{4 \ell^{4}}+E I_{y} N_{P} \frac{\pi^{6}}{4 \ell^{4}}\left(e_{0}^{2}-i_{z}^{2}\right)-E I_{y} d \cdot p \cdot \frac{\pi^{3}}{\ell^{2}}-F_{0}^{2} \cdot z_{1}^{2} \frac{\pi^{4}}{4 \ell^{2}}=0
$$

Tem-se então, uma equação do $2^{\circ}$ grau em $\mathrm{p}^{*}$ a ser desenvolvida:

$$
\begin{gathered}
E I_{y} G J_{t} \frac{\pi^{2}}{2 \ell^{2}}+E I_{y} N_{p} \frac{\pi^{2}}{2 \ell^{2}}\left(e_{0}^{2}-i_{z}^{2}\right)-2 E I_{y} d^{\cdot} \cdot \frac{p^{\cdot}}{\pi}-\frac{p^{.2} z_{1}^{2} \ell^{2}}{8 \operatorname{tg}^{2} \gamma}=0 \\
\frac{\ell^{2} z_{1}^{2}}{8 \operatorname{tg}^{2} \gamma} p^{\cdot 2}+\frac{2 E I_{y} d^{*}}{\pi} p^{\cdot}-\frac{E I_{y} \pi^{2}}{2 \ell^{2}}\left[G J_{t}+N_{p}\left(e_{0}^{2}-i_{z}^{2}\right)\right]=0
\end{gathered}
$$

Extraindo $\mathrm{p}^{*}$ da equação acima, resulta:

$$
p_{c r t i}=\frac{8 E I_{y} d^{\cdot} \operatorname{tg}^{2} \gamma}{\pi \ell^{2} z_{1}^{2}}\left[-1+\sqrt{1+\frac{\pi^{4} z_{1}^{2}}{16 E I_{y} \operatorname{tg}^{2} \gamma d^{2}}\left[G J_{t}+N_{P}\left(e_{0}^{2}-i_{z}^{2}\right)\right]}\right]
$$

Desenvolvendo em série, a expressão de $\mathrm{p}_{\text {crit }}^{*}$, pode-se escrever:

$$
\begin{gathered}
p_{\text {crit }}^{\cdot}=\frac{8 E I_{y} d^{\cdot} \operatorname{tg}^{2} \gamma}{\pi \ell^{2} z_{1}^{2}}\left[\frac{1}{2} \frac{\pi^{8} z_{1}^{4}}{16^{2} E I_{y}^{2} \operatorname{tg}^{4} \gamma d^{4}}\left[G J_{t}+N_{P}\left(e_{0}^{2}-i_{z}^{2}\right)\right]^{2}\right] \\
p_{\text {crtt }}^{*}=\frac{\pi^{7} z_{1}^{2}}{64 E I_{y} d^{3} \operatorname{tg}^{2} \gamma \ell^{2}}\left[G J_{t}+N_{P}\left(e_{0}^{2}-i_{z}^{2}\right)\right]^{2}
\end{gathered}
$$

onde a expressão (3.87) determina o carregamento crítico através da definição de p em séries de Fourier, na equação (2.75).

\subsection{APLICAÇÃo DO MÉTODO DE RUNGE-KUTTA EM PROBLEMAS DE INSTABILIDADE LATERAL DE VIGAS EM SUSPENSÃO}

\subsubsection{INTRODUÇÃO}

O estudo da instabilidade lateral de vigas esbeltas de seção constante, 
retangulares e duplo $\mathrm{T}$ simétrico, suspensas por cabos presos às extremidades com inclinação variável, foi feito através da programação do método numérico de RungeKutta, por representar algumas situações práticas. Os programas desenvolvidos, para cabos retos e inclinados, calculam a carga ou o comprimeno crítico, a partir das equações diferenciais regentes da instabilidade lateral para carregamento linear distribuído no eixo baricêntrico.

As situações analisadas foram as seguintes:

. viga de seção retangular suspensa por cabos verticais e por cabos inclinados; . viga de seção duplo T simétrico suspensa por cabos verticais e por cabos inclinados.

As análises para cabos inclinados representam o caso geral em relação à suspensão por cabos retos. Entretanto, o estudo da instabilidade na suspensão com cabos retos foi desenvolvido para propiciar a verificação dos resultados, na inclinação dos cabos à $90^{\circ}$.

As equações diferenciais que representam as situações acima definidas variam de segunda à oitava ordem.

O algoritmo consiste em transformar uma equação diferencial de n-ésima ordem em $n$ equações diferenciais de $1^{a}$ ordem. A partir das condições de contorno, das características geométricas da seção e das propriedades dos materiais, calculam-se os incrementos definidos pelo método, em cada trecho em que a viga foi dividida. Para as várias iterações, até que o carregamento ou o comprimento representem o primeiro modo de flamgabem, ou seja, indiquem mudança de equilíbrio, pesquisa-se o fenômeno da instabilidade.

O método numérico de Runge-Kutta segundo ABRAMOWITZ \& SEGUN (1968) e KREYSZIG (1988) encontra-se descrito no Anexo A, incluindo as listagens dos programas desenvolvidos para este trabalho.

Os programas elaborados são exclusivamente eficientes para seções delgadas, sendo as vigas metálicas e as vigas de argamassa armada perfeitamente avaliadas pelo processo automatizado. Já os elementos pré-moldados de concreto armado, cujas seções apresentem alta rigidez à torção e à flexão lateral, o programa não apresenta resultados coerentes. 


\subsubsection{PRELIMINARES DO MÉTODO DE RUNGE-KUTTA EM PROBLEMAS DE AUTO-VALOR}

De forma geral, os programas desenvolvidos calculam para uma série de valores do carregamento $\mathrm{p}$ uniformemente distribuído no eixo baricêntrico, o determinante representativo da verificação das condições de contorno. Esta verificação ocorre, geralmente, no meio do vão ou na extremidade da viga. Repetidamente, para cada valor do carregamento incrementado, calcula-se o determinante. $O$ valor do carregamento que faz o determinante igual a zero, é o valor crítico procurado.

As funções diferenciais de ordem $\mathrm{n}$ das equações regentes da instabilidade e das condições de contorno, na tarefa de representar uma equação de ordem $n$ em $n$ equações de ordem 1, devem ser associadas a funções $\mathrm{F}$ de primeira ordem.

O método de Runge-Kutta calcula por passos, as funções $\mathrm{F}$ em cada trecho no qual a viga foi previamente dividida.

Assim, as tabelas 3.2 à 3.4 ilustram as associações feitas entre as funções de ordem $\mathrm{n}$ e as funções $\mathrm{F}$ de primeira ordem, para os tipos de seções e içamento em estudo. À cada função F atribui-se uma variável, a qual será utilizada como incremento. Estes incrementos são quantidades auxiliares que compõem a formulação de RungeKutta no processo de resolução do sistema diferencial.

Em problemas de auto-valor, procede-se inicialmente, atribuindo valores às condições de contorno desconhecidas em $\mathrm{x}=0$, e verificando-as em pontos que são conhecidas. Este procedimento está esquematizado nos quadros 3.1 à 3.3 . Alternadamente, faz-se uma das funções desconhecidas igual a 1 e as demais nulas, e posteriormente, a superposição dos efeitos.

O fluxograma apresentado a seguir, refere-se ao cálculo da carga crítica da viga de seção duplo T simétrico suspensa por cabos inclinados e que representa a resolução da equação diferencial de ordem 8 . Os demais programas apresentam procedimento perfeitamente análogo, alterando apenas o número de superposição de efeitos e o número de funções de primeira ordem.

Para o cálculo do comprimento crítico, o carregamento $\mathrm{p}$ uniformemente 


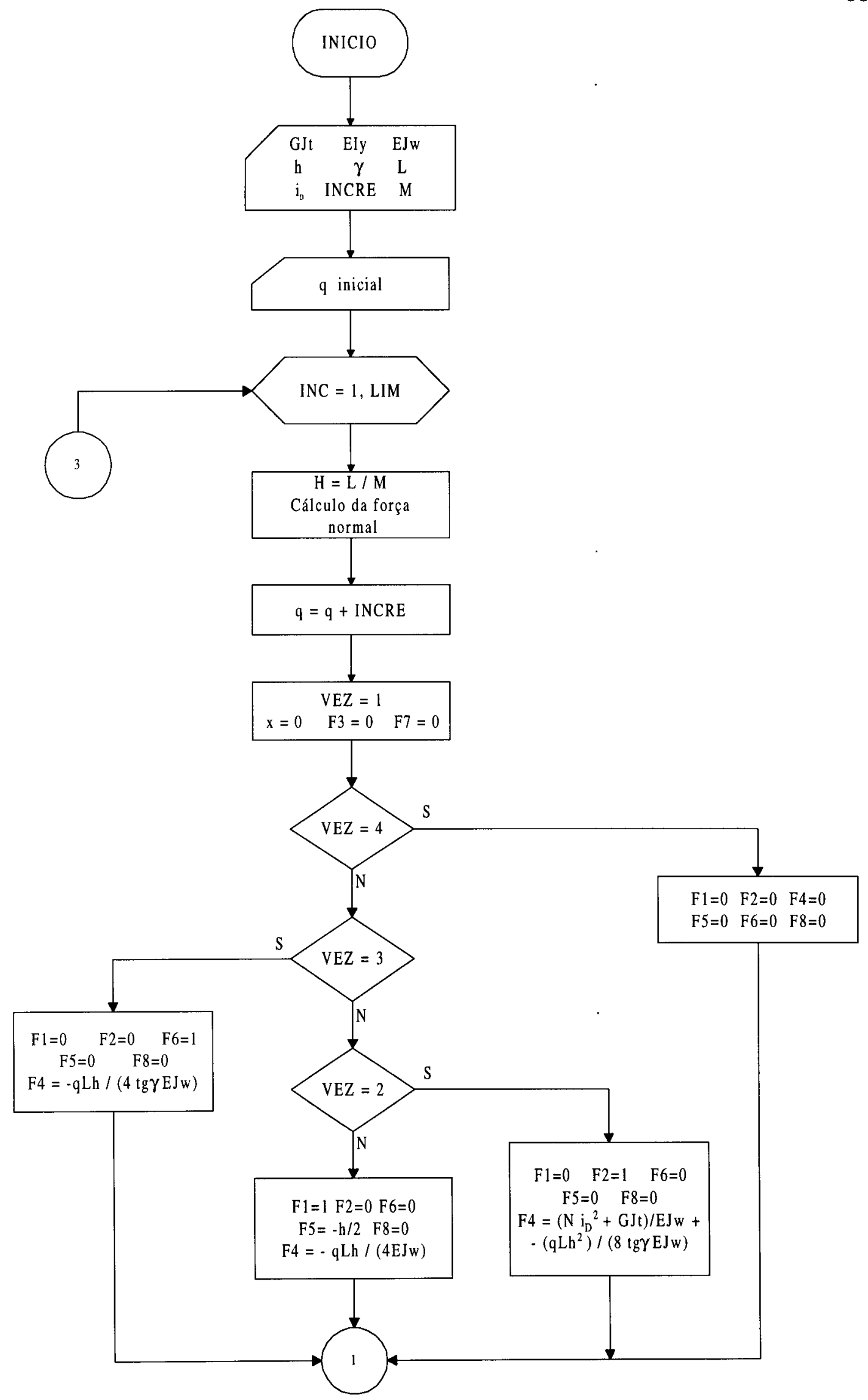




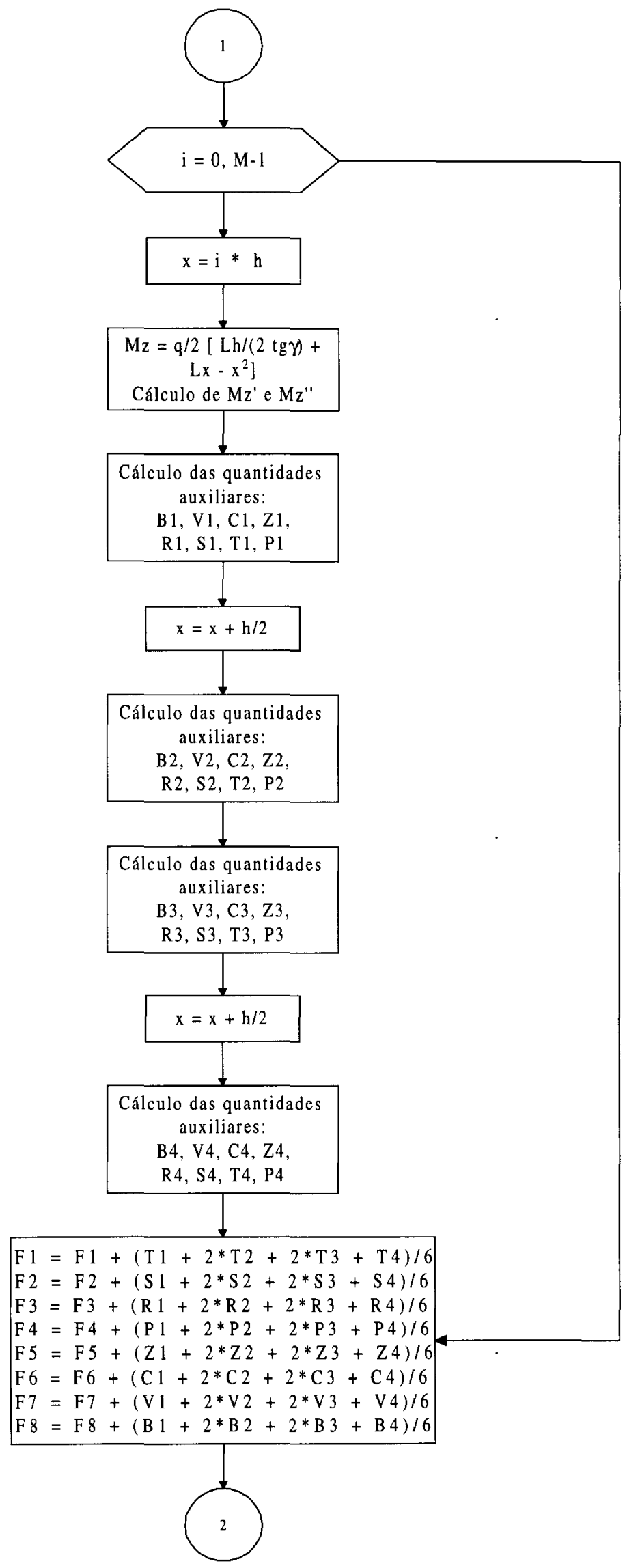




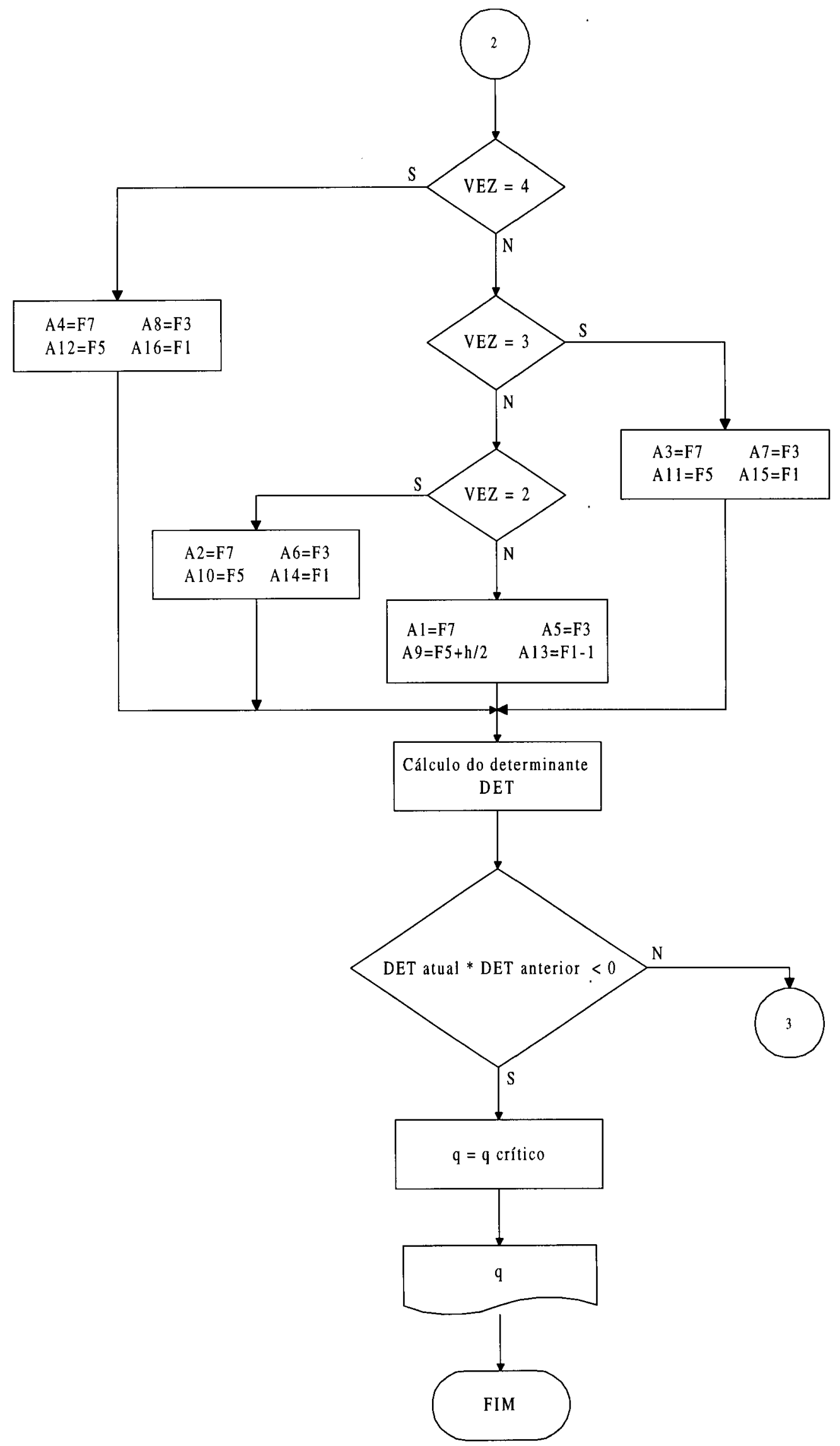


distribuído passa a ser um dado de entrada. O comprimento inicial de $\mathrm{L}$ deve ser sucessivamente incrementado. Para cada valor de $\mathrm{L}$, calcula-se o determinante. O comprimento $\mathrm{L}$ que anula o determinante é o $\mathrm{L}_{\text {crit }}$ da viga em suspensão.

\subsubsection{VIGA DE SEÇÃO RETANGULAR SUSPENSA POR CABOS VERTICAIS}

A viga de seção retangular suspensa nas extremidades por cabos verticais encontra-se ilustrada na figura 3.16. Os procedimentos para a aplicação do método numérico serão descritos a seguir.

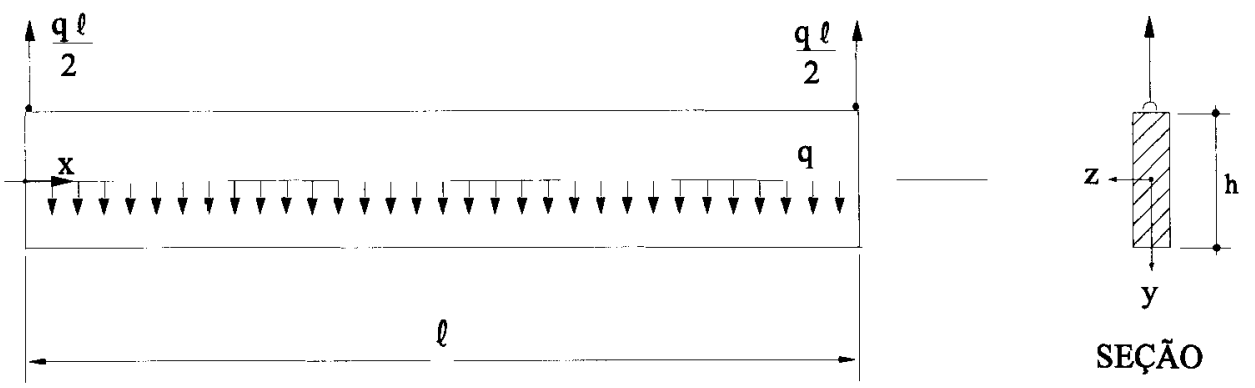

Figura 3.16 - Viga de seção retangular suspensa por cabos verticais.

Em uma seção duplamente simétrica, da geometria resulta:

$$
\begin{aligned}
& y_{D}=z_{D}=0 \\
& K_{y}=K_{z}=0 \quad \text { ( por simetria) }
\end{aligned}
$$

onde $\mathrm{y}_{\mathrm{D}}$ e $\mathrm{z}_{\mathrm{D}}$ são as coordenadas do centro de torção em relação ao $\mathrm{CG}$ da seção e $\mathrm{K}_{\mathrm{y}} \mathrm{e}$ $\mathrm{K}_{\mathrm{z}}$ são as coordenadas do centro do círculo de estabilidade, com dimensão de comprimento.

As equações diferenciais regentes da instabilidade podem ser escritas por:

$$
\begin{aligned}
& \text { (a) } E I_{z} v_{D}^{\prime \prime \prime \prime}-q=0 \\
& \text { (b) } E I_{y} w_{D}^{\prime \prime \prime \prime}+\left(M_{z} \phi\right)^{\prime \prime}=0 \\
& \text { (c) }-G J_{t} \phi^{\prime \prime}+M_{z} w_{D}^{\prime \prime}=0
\end{aligned}
$$


e os esforços de $1^{\text {a }}$ ordem:

$$
\begin{aligned}
& M_{y}=0 \\
& M_{z}=\frac{q \ell}{2} x-\frac{q}{2} x^{2}=\frac{q}{2} x(l-x) \\
& N=0
\end{aligned}
$$

As condições de contorno da viga suspensa por cabos podem ser escritas como segue:

$$
\begin{aligned}
& \text { a) } w_{D}^{\prime \prime}(0)=0 \\
& \text { b) } w_{D}^{\prime \prime}(\ell)=0 \\
& \text { c) } \phi^{\prime}(0)=\frac{q \frac{\ell}{2} \phi(0) \frac{h}{2}}{G J_{t}} \\
& \text { d) } \phi^{\prime}(\ell)=\frac{-q \frac{\ell}{2} \phi(\ell) \frac{h}{2}}{G J_{t}} \\
& \text { e) } \phi(0)=\phi(\ell) \text { ou } \phi^{\prime}\left(\frac{\ell}{2}\right)=0
\end{aligned}
$$

Fazendo a integração preliminar da equação (3.89b), resulta:

$$
\begin{aligned}
& E I_{y} w_{D}^{\prime \prime \prime}+\left(M_{z} \phi\right)^{\prime}=A_{1} \\
& E I_{y} w_{D}^{\prime \prime}+M_{z} \phi=A_{1} x+A_{2}
\end{aligned}
$$

Das condições de contorno que determinam $\mathrm{A}_{2}$, tem-se:

$$
\begin{gathered}
\text { Em } x=0: w_{D}^{\prime \prime}(0)=0 \text { e } M_{z}(0)=0 \\
\therefore A_{2}=0
\end{gathered}
$$

e que determinam $A_{1}$ :

$$
\begin{gathered}
\text { Em } x=\ell: \quad w_{D}^{\prime \prime}(\ell)=0 \text { e } M_{z}(\ell)=0 \\
\therefore A_{1}=0
\end{gathered}
$$

A equação diferencial regente da instabilidade lateral resulta em: 


$$
w_{D}^{\prime \prime}(x)=-\frac{M_{z}(x)}{E I_{y}} \phi(x)
$$

Substituindo a equação resultante de $\mathrm{w}_{\mathrm{D}}$ " na equação (3.89c), tem-se:

$$
\phi^{\prime \prime}(x)+\frac{q^{2}}{4 E I_{y} G J_{t}} x^{2}(\ell-x)^{2} \phi(x)=0
$$

Utilizando a variável auxiliar t, adimensional, pode-se escrever as relações das derivadas:

$$
\begin{aligned}
x & =t \ell \\
\frac{d x}{d t} & =\ell \\
\frac{d \phi}{d x} & =\frac{1}{\ell} \frac{d \phi}{d t} \\
\frac{d^{2} \phi}{d x^{2}} & =\frac{1}{\ell^{2}} \frac{d^{2} \phi}{d t^{2}}
\end{aligned}
$$

e a equação diferencial em t:

$$
\frac{\phi^{\prime \prime}(t)}{\ell^{2}}+\frac{q^{2} \ell^{4}}{4 E I_{y} G J_{t}}\left(t-t^{2}\right)^{2} \phi(t)=0
$$

onde pode-se chamar $\beta$ de:

$$
\beta^{2}=\frac{q^{2} \ell^{6}}{4 E I_{y} G J}
$$

A expressão diferencial, definida no intervalo de t entre 0 e $1 / 2$, é:

$$
\phi^{\prime \prime}(t)=-\beta^{2}\left(t-t^{2}\right)^{2} \phi(t)
$$

Para um problema de auto-valor, partindo de uma condição de contorno atribuída, por exemplo $\phi^{\prime}(0)=1$, obtém-se na relação (3.91c), o respectivo valor de $\phi(0)$. A relação entre $\phi$ e $\phi^{\prime}$, para $t=0$ é:

$$
\phi(t=0)=\frac{4 G J_{t}}{q \ell^{2} h} \phi^{\prime}(t=0)
$$

lembrando que $d \phi / d x=(1 / \ell) .(d \phi / d t)$. 
Para a equação diferencial resultante neste item, foi aplicado de forma direta, a solução de Runge-Kutta em equações diferenciais de segunda ordem, como apresentadas em ABRAMOWITZ \& SEGUN (1968). Entretanto, poderia ter sido desenvolvido sem nenhuma dificuldade, como foi feito para as demais resoluções, a associação de uma equação de ordem 2 em duas equações de primeira ordem.

Assim, atribuindo $M$ passos ao processo, o valor de $\beta$ que verifica a condição $\phi^{\prime}(\mathrm{t}=1 / 2)=0$, define a carga crítica ou o vão limite para a viga em fase de suspensão.

\subsubsection{VIGA DE SEÇÃo RETANGULAR SUSPENSA POR CABOS INCLINADOS}

Para a viga de seção retangular em suspensão por cabos inclinados dispostos nas extremidades, conforme ilustra a figura 3.17 , serão desenvolvidos os procedimentos para a programação do cálculo da carga crítica ou do comprimento crítico, através da aplicação do método de Runge-Kutta, na resolução da equação diferencial regente.

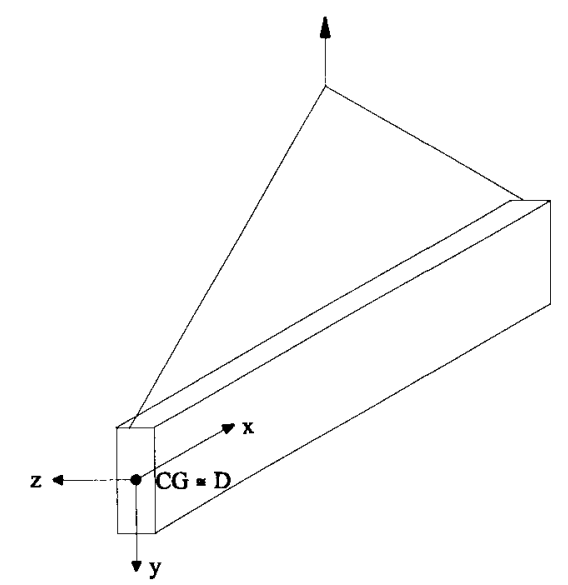

Figura 3.17 - Viga de seção retangular suspensa por cabos inclinados.

A figura 3.18 representa o equilíbrio das forças atuantes na viga em suspensão.

As expressões da força $F_{A}$ de tração do cabo inclinado e sua respectiva componente horizontal $\mathrm{N}_{\mathrm{A}}$ estão definidas pelas equações (3.102) e (3.103). 


$$
\begin{aligned}
& F_{A}=\frac{q \ell}{2 \operatorname{sen} \gamma} \\
& N_{A}=-\frac{q \ell}{2 \operatorname{tg} \gamma}
\end{aligned}
$$

O momento fletor $\mathrm{M}_{\mathrm{z}}$ aplicado na extremidade $\mathrm{A}$ da seção é:

$$
M_{z_{A}}=\frac{q \ell h}{4 \operatorname{tg} \gamma}
$$

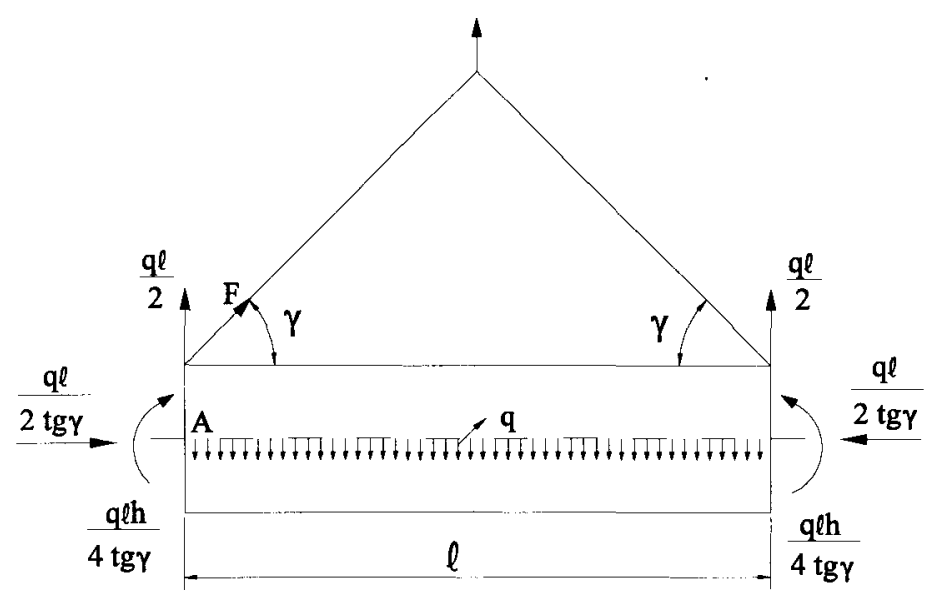

Figura 3.18 - Equilíbrio da viga em suspensão por cabos inclinados

e em uma seção S qualquer:

$$
M_{z_{S}}=\frac{q}{2}\left[\frac{\ell h}{2 \operatorname{tg} \gamma}+\ell x-x^{2}\right]
$$

$\mathrm{O}$ momento fletor $\mathrm{M}_{\mathrm{y}}$ relativo à flexão lateral é nulo nas seções extremas da viga. As equações diferenciais da instabilidade para os esforços ilustrados na figura 3.18 são: 


$$
\begin{aligned}
& \text { (a) } E I_{z} v_{D}^{\prime \prime \prime \prime}-\left(N v_{D}^{\prime}\right)^{\prime}-q=0 \\
& \text { (b) } E I_{y} w_{D}^{\prime \prime \prime \prime}-\left(N w_{D}^{\prime}\right)^{\prime}+\left(M_{z} \phi\right)^{\prime \prime}=0 \\
& \text { (c) }-G J_{i} \phi^{\prime \prime}-N i_{D}^{2} \phi^{\prime \prime}+M_{z} w_{D}^{\prime \prime}=0
\end{aligned}
$$

A resolução da equação diferencial desacoplada (3.106a) referente à flexão no plano vertical será descrita a seguir.

$$
v_{D}^{\prime \prime \prime \prime}-\frac{N}{E I_{z}} v_{D}^{\prime \prime}=\frac{q}{E I_{z}}
$$

A equação para o cálculo da solução homogênea resulta em:

$$
v_{D}^{\prime \prime \prime \prime}(x)+\frac{q \ell}{2 \operatorname{tg} \gamma E I_{z}} v_{D}^{\prime \prime}(x)=0
$$

e assumindo a solução convencional, tem-se:

$$
\begin{gathered}
\left(v_{D}\right)_{h o m}=A_{1}+A_{2} x+A_{3} \operatorname{sen} k_{c} x+A_{4} \cos k_{c} x \\
\text { onde } k_{c}^{2}=\frac{q \ell}{2 \operatorname{tg} \gamma E I_{z}}
\end{gathered}
$$

A expressão da solução particular pode ser escrita por:

$$
v_{D}^{\prime \prime \prime \prime}(x)+k_{c}^{2} v_{D}^{\prime \prime}(x)=\frac{q}{E I_{z}}
$$

Admitindo a equação da elástica em z descrita pelo polinômio abaixo, tem-se as respectivas derivadas:

$$
\begin{aligned}
& v_{D}=A_{5}+A_{6} x+A_{7} x^{2} \\
& v_{D}^{\prime}=A_{6}+2 A_{7} x \\
& v_{D}^{\prime \prime}=2 A_{7} \\
& v_{D}^{\prime \prime \prime}=0 \\
& v_{D}^{\prime \prime \prime \prime}=0
\end{aligned}
$$

Substituindo as expressões das derivadas na equação da solução particular, obtém-se o valor da constante $A_{7}$ : 


$$
A_{7}=\frac{q}{E I_{z}} \frac{1}{2 k_{c}^{2}}
$$

de forma que, como $\mathrm{A}_{6}$ é qualquer, pode ser suposto nulo. Do mesmo modo, procede-se para a constante $\mathrm{A}_{5}$. Então, $\mathrm{A}_{5}=\mathrm{A}_{6}=0$ que satisfaz, e é solução.

Portanto, a solução da particular:

$$
\left(v_{D}\right)_{p a r}=\frac{q}{E I_{z}} \frac{1}{2 k_{c}^{2}} x^{2}
$$

A expressão da solução geral pode ser escrita por:

$$
\begin{gathered}
v_{D}=\left(v_{D}\right)_{h o m}+\left(v_{D}\right)_{p a r} \\
v_{D}(x)=A_{1}+A_{2} x+A_{3} \operatorname{sen} k_{c} x+A_{4} \cos k_{c} x+\frac{q}{E I_{z}} \frac{1}{2 k_{c}^{2}} x^{2}
\end{gathered}
$$

A partir das condições de contorno e assumindo a hipótese de cabos inextensíveis, obtém-se as expressões das constantes de integração, que ficam indeterminadas em função de $\mathbf{k}_{\mathbf{c}}$.

A carga crítica é de $2^{a}$ espécie, ou seja, a posição deslocada é função do carregamento e existe deslocamento para qualquer valor do carregamento diferente de zero. Assim, a carga crítica é aquela que faz os valores dos deslocamentos tenderem a infinito. A elástica tende a infinito quando $\operatorname{sen} \mathrm{k}_{\mathrm{c}} \mathrm{l}=0$.

A expressão da carga crítica de flambagem no plano vertical é:

$$
q_{c r i t}=\frac{2 \pi^{2} \operatorname{tg} \gamma E I_{z}}{\ell^{3}}
$$

As expressões diferenciais (3.107b) e (3.107c) representantes da instabilidade lateral são:

$$
\begin{aligned}
& E I_{y} w_{D}^{\prime \prime \prime \prime}-N w_{D}^{\prime \prime}+M_{z} \phi^{\prime \prime}+2 M_{z}^{\prime} \phi^{\prime}+M_{z}^{\prime \prime} \phi=0 \\
& \phi^{\prime \prime}=\frac{M_{z}}{G J_{t}+N i_{D}^{2}} w_{D}^{\prime \prime}
\end{aligned}
$$


As condições de contorno da viga suspensa por cabos podem ser escritas como segue:

a) $w_{D}^{\prime \prime}(0)=0$

b) $w_{D}^{\prime \prime}(l)=0$

c) $w_{D}(0)=-\frac{h}{2} \phi(0)$

d) $w_{D}(\ell)=-\frac{h}{2} \phi(\ell)$

e) $\phi(0)=\phi(\ell) \quad$ ou $\quad \phi^{\prime}(\ell / 2)=0$

f) $M_{\ell\left(2^{2}\right)}=\left(N i_{D}^{2}+G J_{t}\right) \phi^{\prime}$ e $\quad M_{f t}=0$

$$
\begin{aligned}
& M_{t\left(2^{2}\right)}=\frac{q \ell h}{4} \phi(0)+\frac{q \ell h}{4 \operatorname{tg} \gamma} w_{D}^{\prime}(0)+\frac{q l h^{2}}{8 \operatorname{tg} \gamma} \phi^{\prime} . \\
& \phi^{\prime}=\frac{\frac{q \ell h}{4} \phi(0)+\frac{q \ell h}{4 \operatorname{tg} \gamma} w_{D}^{\prime}(0)}{N i_{D}^{2}+G J_{t}-\frac{q l h^{2}}{8 \operatorname{tg} \gamma}}
\end{aligned}
$$

A figura 3.19 ilustra como obter a expressão do momento de torção definida na equação (3.118f).

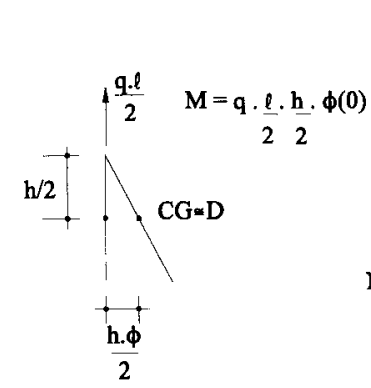

momento de torção devido à componente vertical da força de suspensão

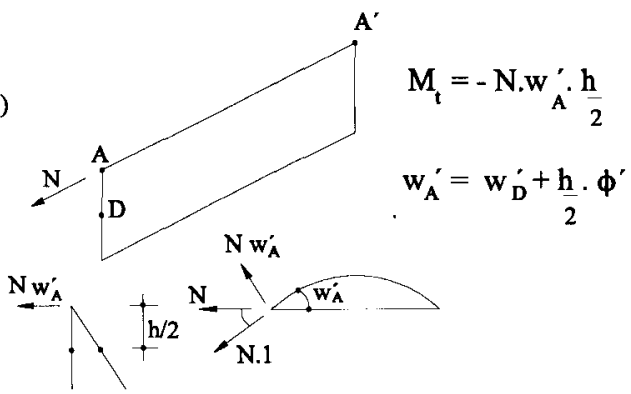

momento de torção devido à componente horizontal da força de suspensão

\section{SEÇÃO EXTREMA ONDE SE FIXAM OS CABOS}

Figura 3.19 - Diagrama para definição da expressão do momento de torção nos apoios.

As expressões do momento fletor $\mathrm{M}_{\mathrm{z}}$ e suas derivadas são: 


$$
\begin{aligned}
& M_{z}=\frac{q}{2}\left[\frac{\ell h}{2 \operatorname{tg} \gamma}+\ell x-x^{2}\right] \\
& M_{z}^{\prime}=\frac{q}{2}[\ell-2 x] \\
& M_{z}^{\prime \prime}=-q
\end{aligned}
$$

A aplicação do método de Runge-Kutta para resolução do sistema de equações diferenciais regentes procede como segue. Ao sistema constituído por uma equação de quarta ordem e outra de segunda ordem, deve-se transformá-las em 6 equações diferenciais de primeira ordem. Para isso, é necessário definir os incrementos de cada função atribuída.

Assim procedendo, a tabela 3.2 contém na $1^{\mathrm{a}}$ coluna, a associação de uma função diferencial de ordem qualquer à uma função $\mathrm{F}$. A segunda coluna da tabela define para cada função F, uma variável representativa do incremento. E por fim, a terceira coluna representa a primeira derivada da função $\mathrm{F}$.

Tabela 3.2 - Expressões diferenciais de $1^{\text {a }}$ ordem - Sistema de ordem 6

\begin{tabular}{||c|c|c||}
\hline Definição & Incremento & Expressão diferencial de $1^{\mathrm{a}}$ ordem \\
\hline$w_{D}^{\prime \prime \prime}=F_{6}$ & $\mathrm{R}$ & $F_{6}^{\prime}=\frac{1}{E I_{y}}\left[\left(N-\frac{M_{z}^{2}}{G J_{t}+N i_{D}^{2}}\right) F_{5}-2 M_{z}^{\prime} F_{2}-M_{z}^{\prime \prime} F_{1}\right]$ \\
\hline$w_{D}^{\prime \prime}=F_{5}$ & $\mathrm{Q}$ & $F_{5}^{\prime}=F_{6}$ \\
\hline$w_{D}^{\prime}=F_{4}$ & $\mathrm{G}$ & $F_{4}^{\prime}=F_{5}$ \\
\hline$w_{D}=F_{3}$ & $\mathrm{D}$ & $F_{3}^{\prime}=F_{4}$ \\
\hline$\phi^{\prime}=F_{2}$ & $\mathrm{C}$ & $F_{2}^{\prime}=\left[\frac{M_{z}}{G J_{t}+N i_{D}^{2}}\right] F_{5}$ \\
\hline$\phi=F_{1}$ & $\mathrm{~B}$ & $F_{1}^{\prime}=F_{2}$ \\
\hline
\end{tabular}


As condições de contorno escritas através das funções definidas na tabela acima são:

$$
\begin{array}{ll}
\mathrm{Em} \mathrm{x}=0: & \mathrm{F}_{5}=0 \\
& \mathrm{~F}_{3}=-\mathrm{h} / 2 \mathrm{~F}_{1}(0) \\
& \mathrm{F}_{1}(0)=\mathrm{F}_{1}(\ell) \\
& \\
& F_{2}=\frac{\frac{q \ell h}{4} F_{1}(0)+\frac{q \ell h}{4 \operatorname{tg} \gamma} F_{4}(0)}{N i_{D}^{2}+G J_{t}-\frac{q \ell h^{2}}{8 \operatorname{tg} \gamma}} \\
& \\
\text { Em x }=\ell: & \mathrm{F}_{5}=0 \\
& \mathrm{~F}_{3}=-\mathrm{h} / 2 \mathrm{~F}_{1}(\ell) \\
& \mathrm{F}_{1}(0)=\mathrm{F}_{1}(\ell)
\end{array}
$$

\begin{tabular}{|c|c|c|c|c|}
\hline \multirow{3}{*}{$\mathrm{VEZ}=1$} & $F_{1}=1$ & \multirow{9}{*}{$\mathrm{F}_{5}=0$} & \multirow{3}{*}{$F_{3}=-h / 2$} & \multirow{3}{*}{$F_{2}=\frac{\frac{q l h}{4}}{N i_{D}^{2}+G J_{t}-\frac{q l h^{2}}{8 \operatorname{tg} \gamma}}$} \\
\hline & $F_{4}=0$ & & & \\
\hline & $\mathrm{F}_{6}=0$ & & & \\
\hline \multirow{3}{*}{ VEZ $=2$} & $\mathrm{~F}_{1}=0$ & & \multirow{3}{*}{$\mathrm{F}_{3}=0$} & $q \ell h$ \\
\hline & $F_{4}=1$ & & & $F_{2}=\frac{4 \operatorname{tg} \gamma}{2}$ \\
\hline & $\mathrm{F}_{6}=0$ & & & $N i_{D}^{2}+G J_{t}-\frac{q l h^{2}}{8 \operatorname{tg} \gamma}$ \\
\hline \multirow{3}{*}{$V E Z=3$} & $\mathrm{~F}_{1}=0$ & & \multirow{3}{*}{$\mathrm{F}_{3}=0$} & \multirow{3}{*}{$F_{2}=0$} \\
\hline & $\mathrm{F}_{4}=0$ & & & \\
\hline & $\mathrm{F}_{6}=1$ & & & \\
\hline
\end{tabular}

$\mathrm{O}$ quadro 3.1 define as funções desconhecidas em $\mathrm{x}=0$, as funç̃os conhecidas e aquelas que lhes são dependentes.

Quadro 3.1 - Procedimentos para superposição de efeitos - Sistema de ordem 6

No quadro acima, tem-se as funções cujos valores são conhecidos em $\mathrm{x}=0$, e as 
funções desconhecidas, representadas pelas funções $\mathrm{F}_{1}, \mathrm{~F}_{4}$ e $\mathrm{F}_{6}$. À estas, deve-se atribuir valores, e por superposição de efeitos, proceder à verificação das condições de contorno em $\mathrm{x}=\ell$.

O trecho de comprimento $\mathrm{H}$ é definido pela razão do vão em relação ao número $M$ de subdivisões da viga: $H=\ell / M$. É intuitivo que esse número $M$ de subdivisões seja diretamente proporcional à precisão do método. Passo à passo, serão calculadas nas 3 vezes necessárias, as funções $\mathrm{F}$ para cada incremento $\mathrm{H}$, até atingir o ponto $\mathrm{x}=\ell$. Neste ponto, as funções devem ser verificadas com as condições de contorno reais por superposição dos efeitos.

As expressões do sistema definido em (3.120) correspondem à verificação das condições de contorno em $\mathrm{x}=\ell$ :

$$
\begin{gathered}
F_{5}(l)=0 \\
\overline{F_{5}} \cdot F_{1}(0)+\overline{\overline{F_{5}}} \cdot F_{4}(0)+F_{6}(0)=0 \\
F_{3}(l)=-\frac{h}{2} F_{1}(0) \\
\overline{F_{3}} \cdot F_{1}(0)+\overline{\overline{F_{3}}} \cdot F_{4}(0)+\overline{\overline{\bar{F}_{3}}} \cdot F_{6}(0)=-\frac{h}{2} F_{1}(0) \\
F_{1}(l)=F_{1}(0) \\
\overline{F_{1}} \cdot F_{1}(0)+\overline{\overline{F_{1}}} \cdot F_{4}(0)+\overline{\overline{F_{1}}} \cdot F_{6}(0)=F_{1}(0)=F_{1}(l)
\end{gathered}
$$

Para o valor da carga ou do comprimento que anulam o determinante abaixo esquematizado, tem-se a situação crítica que define a mudança de equilíbrio.

$$
\left[\begin{array}{lll}
\overline{F_{5}} & \overline{\bar{F}_{5}} & \overline{\overline{F_{5}}} \\
\overline{F_{3}}+\frac{h}{2} & \overline{\bar{F}_{3}} & \overline{\overline{F_{3}}} \\
\overline{F_{1}}-1 & \overline{\overline{F_{1}}} & \overline{\overline{F_{1}}}
\end{array}\right]\left[\begin{array}{l}
F_{1}(0) \\
F_{4}(0) \\
F_{6}(0)
\end{array}\right]=\left[\begin{array}{l}
0 \\
0 \\
0
\end{array}\right]
$$

onde $\bar{F}_{i}, \overline{\bar{F}}_{i} e \overline{\bar{F}}_{i}$ representam o valor da função $F_{i}$ para as 3 superposições de efeitos. 


\subsubsection{VIGA DE SEÇÃO DUPLO T SIMÉTRICO SUSPENSA POR CABOS VERTICAIS}

A viga da seção duplo $\mathrm{T}$ simétrico suspensa por cabos retos presos às extremidades está ilustrada na figura 3.20.

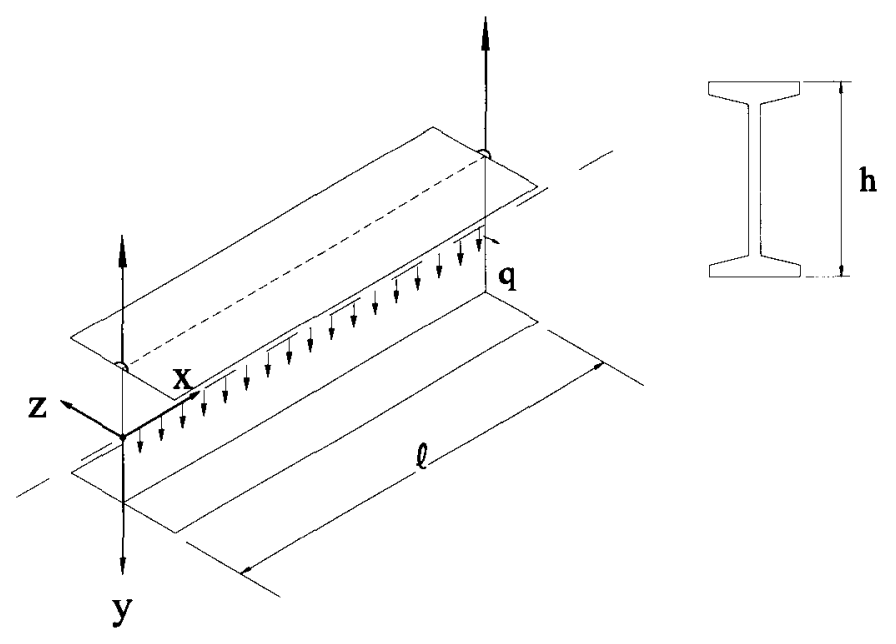

Figura 3.20 - Viga de seção duplo T simétrico suspensa por cabos verticais.

As equações diferenciais regentes da instabilidade lateral para a viga de seção duplo T simétrico, sob carregamento uniforme são:

$$
\begin{aligned}
& \text { (a) } E I_{z} v_{D}^{\prime \prime \prime \prime}-q=0 \\
& \text { (b) } E I_{y} w_{D}^{\prime \prime \prime \prime}+\left(M_{z} \phi\right)^{\prime \prime}=0 \\
& \text { (c) } E J_{w} \phi^{\prime \prime \prime \prime}-G J_{t} \phi^{\prime \prime}+M_{z} w_{D}^{\prime \prime}=0
\end{aligned}
$$

onde o momento fletor $M_{z}$ é dado por:

$$
\begin{aligned}
& M_{z}=\frac{q \ell}{2} x-\frac{q}{2} x^{2} \\
& M_{z}=\frac{q}{2}\left(\ell x-x^{2}\right)
\end{aligned}
$$

Fazendo a integração preliminar da equação (3.122b), tem-se:

$$
E I_{y} w_{D}^{\prime \prime}+M_{z} \phi=C_{1} x+C_{2}
$$


Para as condições de contorno em $\mathrm{x}=0$ e $\mathrm{x}=\ell$, obtém-se os valores das constantes de integração $C_{1}$ e $C_{2}$. Em x=0, $M_{z}=0$ e $w_{D}=0$, e portanto $C_{2}=0$. Da mesma forma para $\mathrm{X}=\ell$, resulta $\mathrm{C}_{1}=0$.

As equações diferenciais finais regentes da instabilidade lateral são:

$$
\begin{aligned}
& w_{D}^{\prime \prime}=-\frac{M_{z}}{E I_{y}} \phi \\
& \phi^{\prime \prime \prime \prime}=\frac{G J_{t}}{E J_{w}} \phi^{\prime \prime}+\frac{M_{z}^{2}}{E J_{w} E I_{\nu}} \phi
\end{aligned}
$$

As condições de contorno em $\phi$ da viga suspensa por cabos podem ser escritas como segue:

$$
\begin{aligned}
& \text { a) } \phi(0)=\phi(l) \\
& \text { b) } \phi^{\prime \prime}(0)=0 \\
& \text { c) } \phi^{\prime \prime}(\ell)=0 \quad \text { ou } \quad \phi^{\prime \prime \prime}(\ell / 2)=0 \\
& \text { d) } M_{t}=M_{\ell}+M_{f t}=G J_{t} \phi^{\prime}-E J_{w} \phi^{\prime \prime \prime} \\
& M_{t}=q \frac{\ell}{2} \frac{h}{2} \phi(0) \\
& \phi^{\prime \prime \prime}=\frac{G J_{t} \phi^{\prime}-\frac{q \ell h}{4} \phi}{E J_{w}}
\end{aligned}
$$

As funções $\mathrm{F}$ e seus respectivos incrementos, para aplicação do método numérico estão definidas na tabela 3.3 .

As condições de contorno escritas através das funções $\mathrm{F}$ definidas na tabela 3.3 são:

$$
\begin{array}{ll}
\mathrm{Em} \mathrm{x}=0: & \mathrm{F}_{2}=0 \\
& F_{1}=\frac{G J_{t} F_{3}-\frac{q \ell h}{4} F_{4}}{E J_{w}} \\
\mathrm{Em} \mathrm{x}=\ell / 2: & \mathrm{F}_{3}=0 \\
& \mathrm{~F}_{1}=0
\end{array}
$$


Tabela 3.3 - Expressões diferenciais de $1^{\text {an }}$ ordem - Sistema de ordem 4

\begin{tabular}{|c|c|c||}
\hline Definição & Incremento & Expressão diferencial de $1^{\mathrm{a}}$ ordem \\
\hline$\phi^{\prime \prime \prime}=F_{1}$ & $\mathrm{~B}$ & $F_{1}^{\prime}=\frac{1}{E J_{w}}\left[G_{t} F_{2}+\frac{M_{z}^{2}}{E I_{y}} F_{4}\right]$ \\
\hline$\phi^{\prime \prime}=F_{2}$ & $\mathrm{C}$ & $F_{2}^{\prime}=F_{1}$ \\
\hline$\phi^{\prime}=F_{3}$ & $\mathrm{D}$ & $F_{3}^{\prime}=F_{2}$ \\
\hline$\phi=F_{4}$ & $\mathrm{G}$ & $F_{4}^{\prime}=F_{3}$ \\
\hline
\end{tabular}

O quadro 3.2 esquematiza as duas etapas do processo de resolução da equação diferencial por Runge-Kutta.

Quadro 3.2 - Procedimentos para superposição de efeitos - Sistema de ordem 4

\begin{tabular}{||c|c|c|c||}
\hline \multirow{2}{*}{ VEZ =1 } & $\mathrm{F}_{3}=1$ & \multirow{3}{*}{$\mathrm{F}_{2}=0$} & $F_{1}=\frac{G J_{t}}{E J_{w}}$ \\
\cline { 2 - 2 } & $\mathrm{F}_{4}=0$ & & $F_{1}=-\frac{q \ell h}{4 E J_{w}}$ \\
\hline VEZ $=2$ & $\mathrm{~F}_{3}=0$ & & \\
\cline { 2 - 3 } & $\mathrm{F}_{4}=1$ & & \\
\hline
\end{tabular}

O sistema de equações para verificação das condições de contorno no meio do vão, em $x=\ell / 2$ é: 


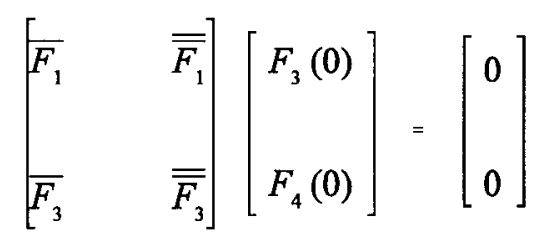

de forma que, para carga e comprimento que anulem o determinante, tem-se a configuração crítica.

\subsubsection{VIGA DE SEÇÃO DUPLO T SIMÉTRICO SUSPENSA NOS EXTREMOS POR CABOS INCLINADOS}

A viga de seção duplo $\mathrm{T}$ simétrico suspensa nas extremidades por cabos inclinados está ilustrada na figura 3.21.

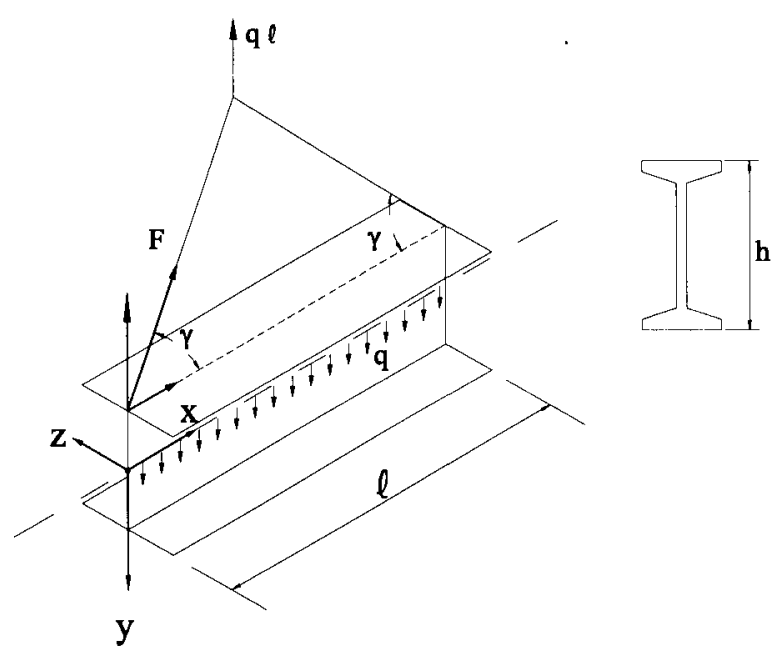

Figura 3.21 - Viga de seção duplo T simétrico suspensa por cabos inclinados.

As equações diferenciais regentes da instabilidade lateral são:
(a) $E I_{z} v_{D}^{\prime \prime \prime \prime}-N v_{D}^{\prime \prime}-q=0$
(b) $E I_{y} w_{D}^{\prime \prime \prime \prime}-N w_{D}^{\prime \prime}+\left(M_{z} \phi\right)^{\prime \prime}=0$
(c) $E J_{w} \phi^{\prime \prime \prime \prime}-G J_{t} \phi^{\prime \prime}-N i_{D}^{2} \phi^{\prime}+M_{z} w_{D}^{\prime \prime}=0$ 
e o sistema de equações acopladas representantes da instabilidade:

$$
\begin{aligned}
& w_{D}^{\prime \prime \prime \prime}=\frac{1}{E I_{y}}\left[N w_{D}^{\prime \prime}-M_{z} \phi^{\prime \prime}-2 M_{z}^{\prime} \phi^{\prime}-M_{z}^{\prime \prime} \phi\right] \\
& \phi^{\prime \prime \prime \prime}=\frac{1}{E J_{w}}\left[\left(N i_{D}^{2}+G J\right) \phi^{\prime \prime}-M_{z} w_{D}^{\prime \prime}\right]
\end{aligned}
$$

As condições de contorno necessárias à aplicação do método numérico são:

a) $w_{D}^{\prime \prime}(0)=0$

b) $w_{D}^{\prime \prime}(\ell)=0$

c) $\phi^{\prime \prime}(0)=0$

d) $\phi^{\prime \prime}(\ell)=0$

e) $w_{D}(0)=-\frac{h}{2} \phi(0)$

f) $w_{D}(\ell)=-\frac{h}{2} \phi(\ell)$

g) $\phi(0)=\phi(l) \quad \therefore \quad \phi^{\prime}(\ell / 2)=0$

h) $M_{\ell\left(2^{\prime}\right)}=\left(N i_{D}^{2}+G J\right) \phi^{\prime} \quad$ e $\quad M_{t}=M_{f t}+M_{\ell}$

$$
\begin{aligned}
& M_{t\left(\left(^{\prime}\right)\right.}=\frac{q \ell h}{4 \operatorname{tg} \alpha} w_{D}^{\prime}(0)+\frac{q l h}{4} \phi(0)+\frac{q l h^{2}}{8 \operatorname{tg} \alpha} \phi^{\prime} \\
& \phi^{\prime \prime \prime}=\frac{\frac{-q \ell h}{4 \operatorname{tg} \alpha} w_{D}^{\prime}(0)-\frac{q l h}{4} \phi(0)-\frac{q l h^{2}}{8 \operatorname{tg} \alpha} \phi^{\prime}+\left(N i_{D}^{2}+G J_{t}\right) \phi^{\prime}}{E J_{w}}
\end{aligned}
$$

As condições de contorno definidas através das funções $F$ podem ser escritas como segue:

$$
\begin{array}{ll}
\operatorname{Em} x=0: & F_{7}=0 \\
& F_{3}=0 \\
& F_{5}=-h / 2 \quad F_{1}(0)
\end{array}
$$




$$
\begin{aligned}
& \mathrm{F}_{1}(0)=\mathrm{F}_{1}(\ell) \\
& F_{4}=\frac{-\frac{q \ell h}{4 \operatorname{tg} \gamma} F_{6}-\frac{q \ell h}{4} F_{1}-\frac{q \ell h^{2}}{8 \operatorname{tg} \gamma} F_{2}+\left(N i_{D}^{2}+G J_{t}\right) F_{2}}{E J_{w}}
\end{aligned}
$$

$$
\begin{array}{ll}
\text { Em } x=\ell: & F_{7}=0 \\
& F_{3}=0 \\
& F_{5}=-h / 2 \quad F_{1}(\ell) \\
& F_{1}(0)=F_{1}(\ell)
\end{array}
$$

A tabela 3.4 representa as expressões diferenciais de ordem 1 relativas ao sistema de equações diferenciais regentes da instabilidade lateral.

Tabela 3.4 - Expressões diferenciais de $1^{\text {a }}$ ordem - Sistema de ordem 8

\begin{tabular}{||c|c|c||}
\hline \hline Definição & Incremento & Expressão diferencial de $1^{\mathrm{a}}$ ordem \\
\hline$w_{D}^{\prime \prime \prime}=F_{8}$ & $\mathrm{~B}$ & $F_{\mathrm{8}}^{\prime}=\frac{1}{E I_{y}}\left[N F_{7}-M_{z} F_{3}-2 M_{z}^{\prime} F_{2}-M_{z}^{\prime \prime} F_{1}\right]$ \\
\hline$w_{D}^{\prime \prime}=F_{7}$ & $\mathrm{~V}$ & $F_{7}^{\prime}=F_{8}$ \\
\hline$w_{D}^{\prime}=F_{6}$ & $\mathrm{C}$ & $F_{6}^{\prime}=F_{7}$ \\
\hline$w_{D}=F_{5}$ & $\mathrm{Z}$ & $F_{5}^{\prime}=F_{6}$ \\
\hline$\phi^{\prime \prime \prime}=F_{4}$ & $\mathrm{P}$ & $F_{4}^{\prime}=\frac{1}{E J_{w}}\left[\left(N i_{D}^{2}+G J_{t}\right) F_{3}-M_{z} F_{7}\right]$ \\
\hline$\phi^{\prime \prime}=F_{3}$ & $\mathrm{R}$ & $F_{3}^{\prime}=F_{4}$ \\
\hline$\phi^{\prime}=F_{2}$ & $\mathrm{~S}$ & $F_{2}^{\prime}=F_{3}$ \\
\hline$\phi^{\prime}=F_{1}$ & $\mathrm{~T}$ & $F_{1}^{\prime}=F_{2}$ \\
\hline
\end{tabular}


Quadro 3.3 - Procedimentos para superposição de efeitos - Sistema de ordem 8

\begin{tabular}{|c|c|c|c|c|}
\hline \multirow{2}{*}{$\mathrm{VEZ}=1$} & $\mathrm{~F}_{1}=1$ & $\mathrm{~F}_{6}=0$ & \multirow{8}{*}{$\begin{array}{l}\mathrm{F}_{3}=0 \\
\mathrm{~F}_{7}=0\end{array}$} & \multirow{2}{*}{$\begin{array}{l}F_{5}=-\frac{h}{2} \\
F_{4}=-\frac{q \ell h}{4 E J_{w}}\end{array}$} \\
\hline & $\mathrm{F}_{2}=0$ & $\mathrm{~F}_{8}=0$ & & \\
\hline \multirow{2}{*}{$\mathrm{VEZ}=2$} & $\mathrm{~F}_{1}=0$ & $F_{6}=0$ & & $F_{s}=0$ \\
\hline & $\mathrm{F}_{2}=1$ & $\mathrm{~F}_{8}=0$ & & $F_{4}=-\frac{q l h^{2}}{8 \operatorname{tg} \alpha E J_{w}}+\frac{N l_{D}+G J_{t}}{E J_{w}}$ \\
\hline \multirow{2}{*}{$\mathrm{VEZ}=3$} & $\mathrm{~F}_{1}=0$ & $F_{6}=1$ & & \multirow{2}{*}{$\begin{array}{l}F_{5}=0 \\
F_{4}=-\frac{q \ell h}{4 \operatorname{tg} \alpha E J_{w}}\end{array}$} \\
\hline & $\mathrm{F}_{2}=0$ & $\mathrm{~F}_{8}=0$ & & \\
\hline \multirow{2}{*}{$\mathrm{VEZ}=4$} & $F_{1}=0$ & $\mathrm{~F}_{6}=0$ & & \multirow{2}{*}{$\begin{array}{l}F_{5}=0 \\
F_{4}=0\end{array}$} \\
\hline & $\mathrm{F}_{2}=0$ & $F_{8}=1$ & & \\
\hline
\end{tabular}

A verificação das condições de contorno em $\mathrm{x}=\ell$, conforme o quadro 3.3 referente à superposição dos efeitos, deve ser feita igualando a zero o determinante da matriz abaixo definida.

$$
\left[\begin{array}{llll}
\overline{F_{1}}-1 & \overline{\overline{F_{1}}} & \overline{\overline{F_{1}}} & \overline{\overline{\bar{F}}} \\
\overline{F_{3}} & \overline{\overline{F_{3}}} & \overline{\overline{F_{3}}} & \overline{\overline{\overline{F_{3}}}} \\
\overline{F_{5}}+\frac{h}{2} & \overline{\overline{F_{5}}} & \overline{\overline{\bar{F}}} & \overline{\overline{\overline{F_{5}}}} \\
\overline{F_{7}} & \overline{\overline{F_{7}}} & \overline{\overline{F_{7}}} & \overline{\overline{\overline{F_{7}}}}
\end{array}\right]\left[\begin{array}{l}
F_{1}(0) \\
F_{2}(0) \\
F_{6}(0) \\
F_{8}(0)
\end{array}\right]=\left[\begin{array}{l}
0 \\
0 \\
0 \\
0
\end{array}\right]
$$

Para os valores da carga linear distribuída e do comprimento da viga que anulam o determinante, tem-se a situação crítica de flambagem lateral em estudo. 


\section{PROCEDIMENTOS SIMPLIFICADOS PARA DETERMINAÇÃO DO FATOR DE SEGURANÇA DURANTE A FASE TRANSITÓRIA}

\subsection{PRELIMINARES}

O estudo dos fatores de segurança aplicáveis aos problemas de instabilidade lateral na fase transitória apresenta, basicamente, um caráter prático. Até agora, as análises da instabilidade limitaram-se, neste trabalho, ao cálculo do carregamento crítico que provoca mudança de equilíbrio em teoria de segunda ordem. Nenhuma referência à consideração das excentricidades laterais por imperfeições construtivas ou outro motivo qualquer foi feita.

Neste capítulo, será apresentado um estudo simplificado, baseado no trabalho de MAST (1993), para avaliação da segurança das fases transitórias de manuseio e de transporte, considerando as excentricidades geradas na fase de fabricação do elemento.

As excentricidades iniciais podem ter causas diversas, como: as imperfeições construtivas das fôrmas de moldagem, a retração diferenciada entre as faces laterais do elemento, os gradientes térmicos, desvios de posicionamento dos cabos de protensão, 
etc. Tais variações causam curvatura horizontal no elemento durante a sua fabricação. Durante o manuseio, em uma análise crítica, os desvios de posicionamento dos cabos de içamento aumentam a excentricidade lateral, favorecendo a perda de estabilidade.

Os ensaios em laboratório são importantes para direcionar a escolha dos valores médios que devem ser assumidos para as excentricidades iniciais de fabricação.

O significado físico de um coeficiente de segurança pode ser entendido como um valor prático para verificação ou para dimensionamento. Usar um ou outro fator de segurança, depende da análise crítica e da experiência de cada projetista. Na literatura existem algumas indicações práticas para a segurança da fase de içamento e de transporte, como as apresentadas por IMPER \& LASZLO (1987) e MAST $(1989,1993)$.

O estudo de MAST (1993) sobre o comportamento de vigas de seção I protendidas durante a fase de içamento, verificado por ensaios em escala real, é a principal referência do PCI Design Handbook $4^{\mathrm{a}}$ edição (Precast/Prestressed Concrete Institute). Análises considerando a fissuração e a ruptura foram associadas ao estudo dos fatores de segurança, tanto para a suspensão como para o transporte.

Inclui-se ainda neste capítulo, um recente trabalho desenvolvido por CUNHA (1994), com algumas indicações práticas para a determinação da rigidez à rotação da carroceria do caminhão e a obtenção da superelevação da rodovia.

\subsection{VIGAS PRÉ-MOLdAdAS DE CONCRETO DURANTE FASE DE IÇAMENTO}

Em uma viga suspensa simplesmente por cabos, que são vínculos que não impedem rotações, as seções dos apoios apresentam deslocamentos laterais e giro. Neste caso, o chamado eixo de giro da viga é composto pela linha que passa pelos pontos onde o cabo está ligado ao elemento estrutural, geralmente na face superior do mesmo.

Considerando que na etapa construtiva é praticamente impossível que não ocorram imperfeições, e sendo importante considerar tolerâncias no posicionamento dos cabos, então é razoável assumir uma excentricidade inicial $\mathrm{e}_{i}$ do eixo de giro em relação 
ao centro de gravidade do elemento. Esta excentricidade causa uma rotação inicial $\phi_{\mathrm{i}}$ na viga, resultando em giro de corpo rígido.

Conforme ilustrado na figura $4.1 \mathrm{~b}$, na posição de equilíbrio, a componente do carregamento Psen $\phi_{\mathrm{i}}$ é responsável por deslocamentos laterais que afasta o centro de massa da viga até a acomodação em um ângulo $\phi$. A componente da força resultante devido ao peso próprio na direção do eixo de maior inércia Psen $\phi$, gera novos deslocamentos laterais na viga. O ciclo, aumento do ângulo $\phi$, que provoca acréscimo da componente lateral devido ao peso próprio, aumentanto os deslocamentos laterais e, consequentemente, incrementando a rotação $\phi$, se inicia. Dependendo da rigidez lateral da viga, esta pode encontrar o equilíbrio para $\phi$ um pouco maior que $\phi_{\mathrm{i}}$, ou então, $\phi$ irá aumentar até o colapso da viga.

(a) Perspectiva de uma viga livre para girar e

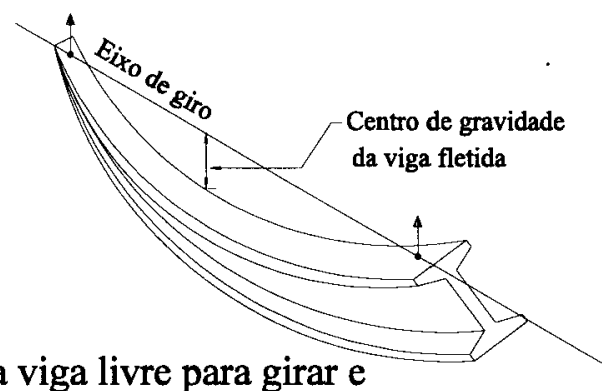

fletir lateralmente

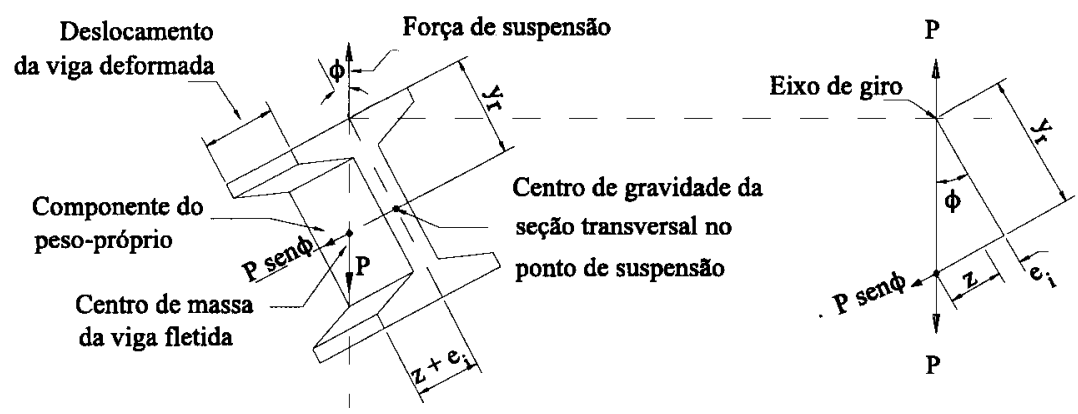

$\begin{array}{ll}\text { (b) Vista em corte } & \text { (c) Diagrama de equilíbrio }\end{array}$

Figura 4.1 - Equilíbrio da viga durante a suspensão. [ MAST (1989) ] 
Assumindo a posição de equilíbrio para toda a viga girada uniformemente de um ângulo $\phi$, a componente Psen $\phi$ causa um deslocamento lateral adicional $\mathrm{z}$ do centro de massa da viga, que passa a apresentar uma configuração curva. De acordo com o diagrama de equilíbrio, figura 4.1c, para o cálculo do ângulo $\phi$, precisa-se conhecer o deslocamento z. Este último depende da componente Psen $\phi$, que por sua vez depende de $\phi$. Tem-se então, uma relação de dependência entre z e $\phi$.

Este impasse pode ser resolvido calculando, primeiramente, o deslocamento teórico $z_{0}$ do centro de massa da viga para todo o peso próprio aplicado lateralmente.

Chamando $\mathrm{z}_{0}$ de um valor fictício, pode-se escrever:

$$
\int_{0}^{1} w_{D}(x) d x=z_{0} \cdot \ell
$$

onde a integral equivale à área da elástica para carregamento uniforme distribuído, $\ell$ é o comprimento da viga e $\mathrm{w}_{\mathrm{D}}$ é o deslocamento lateral do centro de torção na direção do eixo $\mathrm{z}$.

Da equação diferencial da flexão no plano do carregamento, tem-se:

$$
E I_{y} w_{D} \cdots+p=0
$$

onde p é o carregamento uniformemente distribuído.

A solução da equação (4.2) resulta em:

$$
w_{D}(x)=\frac{p}{24 E I_{y}}\left(x^{4}-2 \ell x^{3}+x \ell^{3}\right)
$$

Assim, $\mathrm{z}_{0}$ pode ser definido como:

$$
\begin{gathered}
z_{0}=\frac{\int_{0}^{1} \frac{p}{24 E I_{y}}\left(x^{4}-2 \ell x^{3}+x \ell^{3}\right) d x}{\ell} \\
z_{0}=\frac{p \ell^{4}}{120 E I_{y}}
\end{gathered}
$$

$O$ valor fictício $z_{0}$ pode ser relacionado com $o$ valor da flecha no meio do vão como indicado a seguir: 


$$
\frac{z_{0}}{w_{D}(\ell / 2)}=\frac{\frac{p \ell^{4}}{120 E I_{y}}}{\frac{5 p \ell^{4}}{384 E I_{y}}}=\frac{1}{120} \frac{384}{5}=0,64
$$

Logo:

$$
z_{0} \cong 0,64 w_{D}(\ell / 2)
$$

Enfim, na seção do meio do vão, se z é o deslocamento devido a Psen $\phi$ e $z_{0}$ é o deslocamento devido a P total, então:

$$
z=z_{0} \operatorname{sen} \phi
$$

onde $\mathrm{z}$ e $\mathrm{z}_{0}$ são perpendiculares ao eixo de menor inércia no mesmo plano.

$\mathrm{O}$ valor fíctício $\mathrm{z}_{0}$ é assim chamado, porque a maioria das vigas romperiam quando todo o peso próprio estivesse aplicado lateralmente. A determinação de $\mathrm{z}_{0}$ tem a finalidade de proporcionar o cálculo do deslocamento $\mathrm{z}$.

De acordo com a figura 4.1, pode-se escrever a seguinte equação, relativa à posição $\phi$ de equilíbrio:

$$
\operatorname{tg} \phi=\frac{\left(z_{0} \operatorname{sen} \phi+e_{i}\right)}{y_{r}}
$$

Para uma dada viga, com vão e altura conhecidos, $\mathrm{z}_{0}$ pode ser calculado pela expressão (4.7) e uma excentricidade inicial $e_{i}$ pode ser admitida. Por tentativas, determina-se a rotação $\phi$. Para a maioria das aplicações, $\phi$ é suficientemente pequeno, da ordem de $0,2 \operatorname{rad}$ ou $11,5^{\circ}$. Desta forma, a aproximação para ângulos pequenos, $\phi \sim \operatorname{sen} \phi \sim \operatorname{tg} \phi$, é razoável e pode ser feita. Para $\phi_{\mathrm{i}}=\mathrm{e}_{\mathrm{i}} / \mathrm{y}_{\mathrm{r}}$, tem-se:

$$
\phi=\phi_{i}\left(\frac{1}{1-z_{0} / y_{r}}\right)
$$

Entretanto, ainda que as imperfeições iniciais sejam praticamente nulas, o ângulo $\phi$ tende a infinito quando $\mathrm{z}_{0}$ é aproximadamente igual a $\mathrm{y}_{\mathrm{r}}$, o que pode ser notado na expressão acima, a qual é válida apenas para ângulos pequenos, conforme já definido.

MAST (1989) quantificou inicialmente, os fatores de segurança da seguinte 
maneira: como na equação (4.10), que define $\phi$ em função de $\phi_{\mathrm{i}}$, observa-se que a altura do eixo de giro $\mathrm{y}_{\mathrm{r}}$ precisa ser muito maior que $\mathrm{z}_{0}$, então a razão $\mathrm{y}_{\mathrm{r}} / \mathrm{z}_{0}$ pode ser utilizada na determinação de um fator de segurança contra o colapso por instabilidade lateral.

$$
F S=\frac{y_{r}}{z_{0}}
$$

Esta equação fornece o fator de segurança contra instabilidade lateral para uma viga quase perfeita. Vigas com imperfeições iniciais são mais críticas, e o máximo ângulo $\phi$ que a viga pode tolerar depende da rigidez à flexão lateral. Este ângulo é definido como $\phi_{\text {máx }}$ e a relação $\left(\mathrm{y}_{\mathrm{r}} / \mathrm{z}_{0}\right)_{\text {crítica }}$ é definida pelo valor $\left(\mathrm{y}_{\mathrm{r}} / \mathrm{z}_{0}\right)$ que faz $\phi=\phi_{\operatorname{máx}}$.

Da equação (4.10):

$$
\left(\frac{y_{r}}{z_{0}}\right)_{\text {crit }}=\frac{1}{1-\phi_{i} / \phi_{\text {max }}}
$$

onde, para $\phi$ igual a $\phi_{\text {máx }}$, tem-se a relação $\left(\mathrm{y}_{\mathrm{r}} / \mathrm{z}_{0}\right)$ crítica, podendo-se também avaliar a segurança pela expressão (4.13).

$$
F S=\frac{y_{r} / z_{0}}{\left(y_{r} / z_{0}\right)_{c r t i c o}}
$$

Substituindo a equação (4.12) na expressão (4.13), resulta:

$$
F S=\frac{y_{r}}{z_{0}}\left(1-\frac{\phi_{i}}{\phi_{\max }}\right)
$$

Quando não são consideradas as imperfeições iniciais, ou seja $\phi_{\mathrm{i}}=0$, a equação (4.14) reduz-se à equação (4.11). As equações (4.11) e (4.14) definem o fator de segurança dependendo do parâmetro $\mathrm{z}_{0}$, que é função das características mecânicas da seção transversal. O efeito de $\phi_{\mathrm{i}}$ e $\phi_{\text {máx }}$ na equação (4.14) do fator de segurança tem caráter redutor quando as imperfeições iniciais são admitidas.

Se a viga é rígida lateralmente, e portanto $\mathrm{z}_{0}$ é pequeno, o fator de segurança não deve ser tão alto quanto o resultante pela aplicação da equação (4.14). Do mesmo modo, para as vigas rígidas, existe uma tolerância máxima para o ângulo de giro $\Phi_{\max }$, além do qual, a viga pode romper por flexão lateral. Porém, para uma situação onde o efeito da 
excentricidade inicial for dominante, é mais lógico definir o fator de segurança pela relação $\phi_{\text {máx }} / \phi$.

$$
F S=\frac{\phi_{\max }}{\phi}
$$

Substituindo na equação (4.15) o valor de $\phi$ da equação (4.10), tem-se:

$$
F S=\frac{\phi_{\max }}{\phi_{i}}\left(1-\frac{z_{0}}{y_{r}}\right)
$$

A expressão (4.16) é muito parecida com a (4.14), entretanto, varia o parâmetro modificador, representado pelo termo entre parênteses.

$O$ fator de segurança a ser adotado deve ser o menor entre os valores obtidos nas equações (4.14) e (4.16).

Uma comparação com o PCI Design Handbook $3^{\mathrm{a}}$ edição, o qual baseia-se nas considerações definidas em MAST (1989), mostra que o fator de segurança obtido da expressão apresentada no PCI é em torno de $2 / 3$ do fator de segurança dado pela equação (4.11). Entretanto, os resultados entre as duas indicações acima citadas são muito próximos quando ao invés de comparar com o valor dado pela equação (4.11), verificar com o resultante da equação (4.14). A expressão para cálculo do fator de segurança dada no PCI é:

$$
F S=\frac{y_{r}}{w_{D}(\ell / 2)}
$$

O fator de segurança FS dado pela expressão do PCI, de certa forma compensa a ausência de especificações referente às imperfeições e aos desvios.

A consideração das excentricidades iniciais pode ser decisiva na análise da estabilidade lateral, e seus efeitos podem ser avaliados explicitamente pelo uso da equação (4.14).

Inicialmente, os estudos desenvolvidos em MAST (1989) limitavam-se aos problemas de instabilidade antes da viga começar a fissurar, e o máximo ângulo de giro era limitado pela máxima tensão de tração das fibras superiores da seção. Análises 
experimentais desenvolvidas no trabalho de MAST (1993) confirmaram que as vigas suportam ângulos maiores que o ângulo limite de fissuração, mas a rigidez deve ser reduzida. Desta forma, dois ângulos limites podem ser avaliados, um deles é o ângulo de inclinação de fissuração $\phi_{\text {máx }}$, o outro, é o ângulo de inclinação de ruptura $\phi_{\text {máx }}^{\prime}$ É oportuno lembrar que o termo ruptura, tratado pelo autor em questão, não equivale ao estado limite último definido pela norma brasileira.

Pela análise dos resultados de um programa computacional, com comprovação experimental de um ensaio em escala real, MAST (1993), para nove diferentes vigas longas de seção I semelhantes ao padrão PCI BT (bulb tee), verifica que a rigidez à flexão lateral diminui na viga fissurada.

Todas as vigas avaliadas pelo programa quanto à rigidez lateral efetiva, apresentavam o menor momento de inércia em torno de $4 \%$ do maior, e tensão média de protensão mínima aproximadamente igual a $8,30 \mathrm{MPa}$.

Verificou-se similar relação entre a rigidez e o ângulo de inclinação nas nove vigas avaliadas, e o ângulo previsto para atingir a resistência nominal ocorreu em torno de $23^{\circ}$.

A partir destes resultados, MAST (1993) propõe uma expressão simplificada para o cálculo da rigidez efetiva de longas vigas I de concreto protendido de dimensões aproximáveis à da viga PCI BT-72, cuja seção transversal encontra-se ilustrada no capítulo 5, item 5.4. As indicações de MAST(1993) para assumir a rigidez efetiva são: . para ângulos de inclinação que produzem tensões de tração na mesa superior menores que $7,5 \sqrt{f_{c}^{\prime}}$, usar toda a inércia I da seção;

. para ângulos de inclinação que produzem tensões de tração na mesa superior maiores que $7,5 \sqrt{f_{c}^{\prime}}$, usar a inércia efetiva:

$$
I_{e f}=\frac{I}{1+2,5 \phi}
$$

onde $\mathrm{f}_{\mathrm{c}}{ }_{\mathrm{c}}$ é a resistência de um corpo de prova cilíndrico de concreto na data do içamento ou transporte. A expressão $7,5 \sqrt{f_{c}^{\prime}}$, utilizada para definição da rigidez a ser considerada, representa o limite da resistência à tração do concreto, conforme indicado 
no $\mathrm{ACI}$ (American Concrete Institute).

O gráfico da figura 4.2 ilustra os resultados da variação da inércia em relação ao giro $\phi$, obtidos computacionalmente por MAST (1993). A curva tracejada representa a equação (4.18) proposta para o cálculo da inércia efetiva.

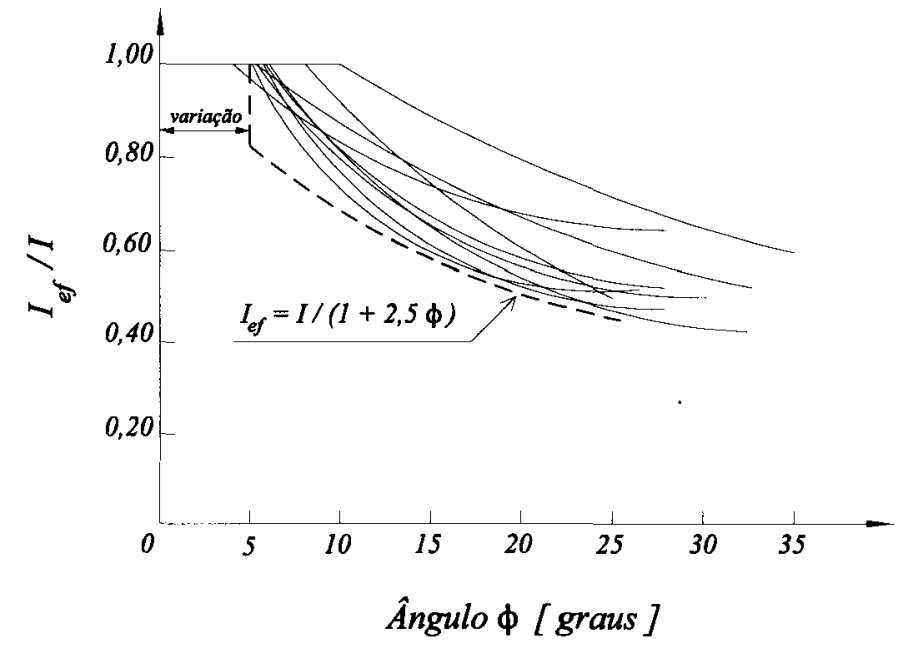

Figura 4.2 - Relação entre a inércia efetiva e o ângulo $\phi$. [ MAST (1993) ]

Embora os resultados apresentados na figura acima, para as nove vigas avaliadas, não indiquem súbita perda de rigidez no início da fissuração, conforme representa a curva tracejada, por outro lado, os deslocamentos também resultam menores após a fissuração. Assim, considerar uma quase instantânea perda de rigidez no início da fissuração é uma hipótese conservativa. O comprimento. do patamar que define a fissuração varia de acordo com a tensão na mesa superior.

Para ângulos de inclinação $\phi$ entre a fissuração e a ruptura, deve-se utilizar a rigidez efetiva, conforme a expressão (4.18), em vigas protendidas de concreto armado, de longos vãos e com dimensões proporcionais à viga PCI BT-72. Para estas vigas, o ângulo de inclinação equivalente à ruptura é da ordem de $0,4 \mathrm{rad}$.

Como $\mathrm{z}_{0}$ é função da rigidez e $\mathrm{I}_{\mathrm{y}}$ depende de $\phi$, então $\mathrm{z}_{0}$ é também função de $\phi$ para as vigas fissuradas. Esta dependência entre $z_{0}$ e $\phi$ torna mais difícil o cálculo de $\phi$, comparado com a solução inicial dada por MAST (1989) para vigas não fissuradas, onde $\mathrm{z}_{0}$ não dependia de $\phi$, visto que o momento de inércia era invariável.

Esta dificuldade pode ser superada plotando as curvas para o braço do momento 
aplicado e o braço de momento resistente. Os braços dos momentos são obtidos dividindo os momentos pelo peso $\mathrm{P}$ total da viga. $\mathrm{O}$ uso dos braços dos momentos ao invés dos momentos foi preferido por MAST (1993) porque elimina o peso próprio $P$ das equações. Além disso, como os braços dos momentos são medidas de comprimento, os gráficos tornam-se mais simples de serem visualizados.

O braço do momento aplicado $\mathrm{c}_{\mathrm{a}}$ é a medida do deslocamento do centro de massa da viga fletida e é dado pela equação (4.19). O braço do momento resistente $\mathrm{c}_{\mathrm{r}}$ é obtido pela expressão (4.20). Os valores de $c_{a}$ e $c_{r}$ são calculados a partir dos momentos em torno do CG na seção transversal do ponto de suspensão. A figura 4.3 ilustra $c_{a}$ e $c_{r}$.

$$
\begin{gathered}
c_{a}=z_{0} \operatorname{sen} \phi \cos \phi+e_{i} \cos \phi \\
c_{r}=y_{r} \operatorname{sen} \phi
\end{gathered}
$$

Aproximando $\operatorname{sen} \phi=\phi$ e $\cos \phi=1$, as curvas que definem $c_{a}$ e $c_{r}$ podem ser representadas por retas, onde a intersecção das mesmas indica o ângulo $\phi$ de equilíbrio. A figura 4.4 ilustra graficamente a relação entre os braços dos momentos e a rotação $\phi$.

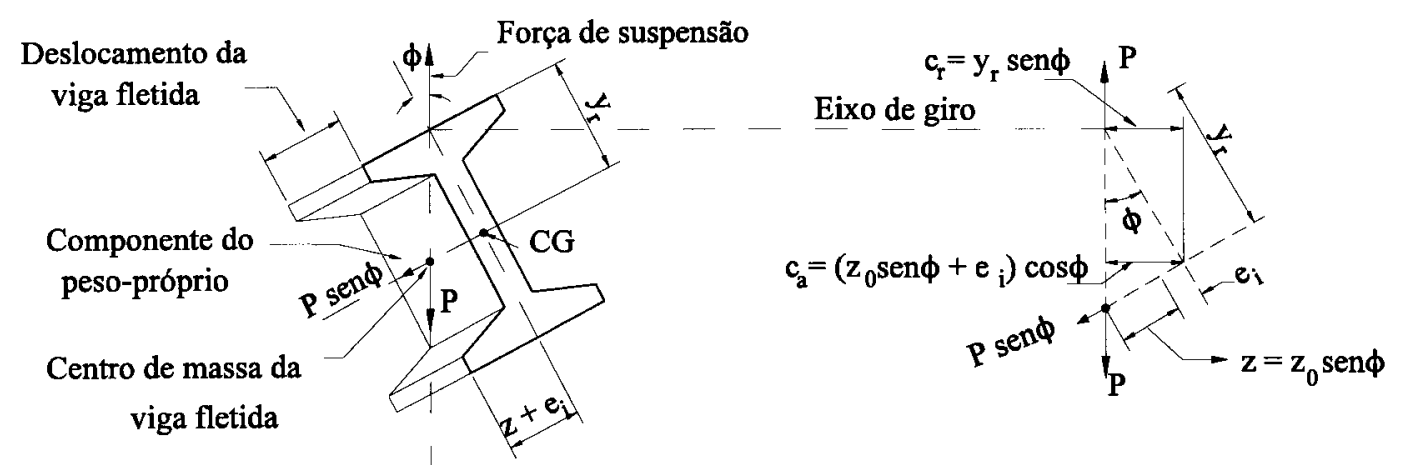

(a) Vista em corte

(b) Diagrama de Equilíbrio

Figura 4.3 - Braço do momento aplicado e do momento resistente. [ MAST (1993) ]

O fator de segurança pode ser definido como a razão entre o braço do momento resistente $c_{\mathrm{r}}$ e o braço do momento aplicado $c_{\mathrm{a}}$ :

$$
F S=\frac{c_{r}}{c_{a}}=\frac{y_{r} \phi}{z_{o} \phi+e_{i}}
$$




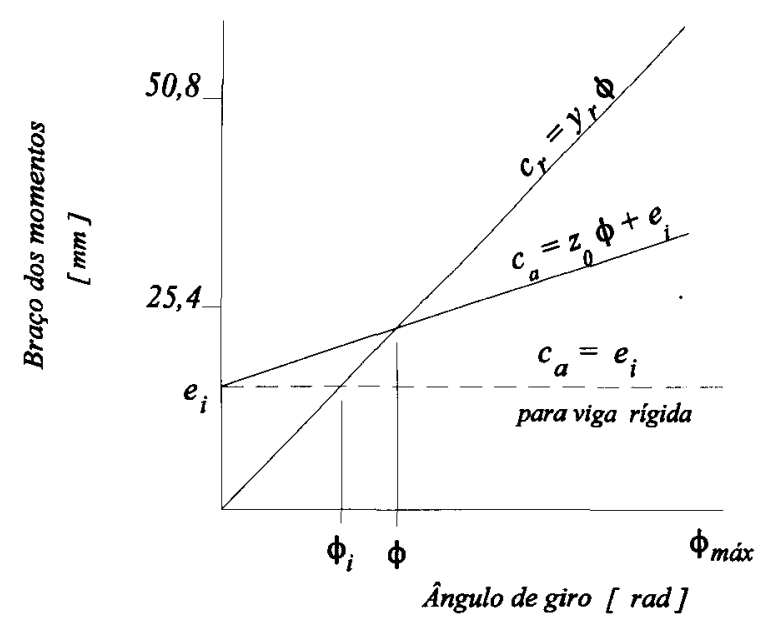

Figura 4.4 - Braço dos momentos $x$ ângulo de inclinação. [ MAST (1993) ]

O ângulo inicial de giro $\phi_{\mathrm{i}}$ da viga como corpo rígido é obtido pela equação $\mathrm{e}_{\mathrm{i}} / \mathrm{y}_{\mathrm{r}}$. Quando $\phi$ é igual a $\phi_{\max }$, tem-se o ângulo de inclinação equivalente à fissuração. Substituindo $\phi_{\mathrm{i}} \mathrm{y}_{\mathrm{r}}=\mathrm{e}_{\mathrm{i}}$ e simplificando, resulta o fator de segurança FS contra fissuração:

$$
F S=\frac{1}{z_{0} / y_{r}+\phi_{i} / \phi_{\max }}
$$

A figura 4.5 representa a equação (4.22) graficamente, que deve ser preferida às equações (4.14) e (4.16), visto que considera o efeito combinado das duas relações $z_{0} / y_{r}$ e $\phi_{\mathrm{i}} / \phi_{\text {máx }}$ variando simultaneamente.

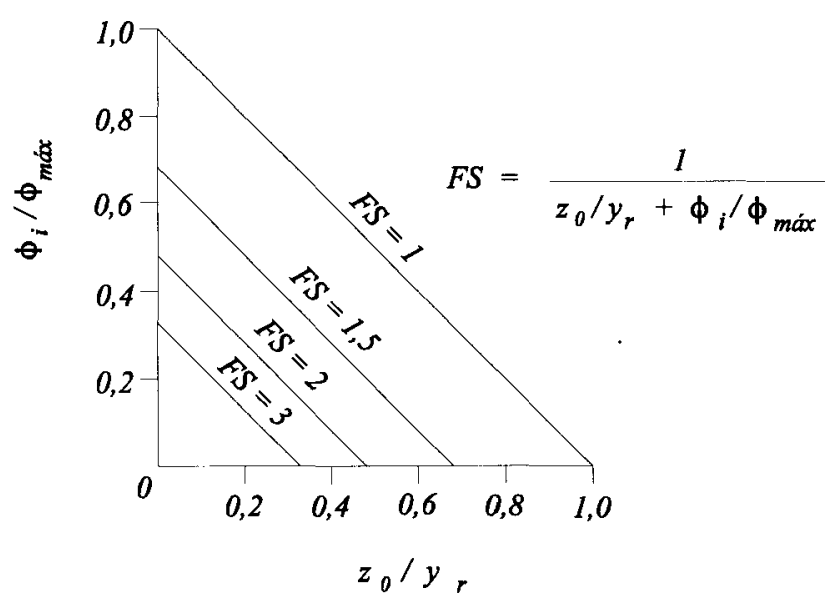

Figura 4.5 - Fatores de segurança contra fissuração. [ MAST (1993) ] 
$\mathrm{O}$ fator de segurança contra ruptura FS' pode ser analisado na figura 4.6, após o ponto de fissuração. A razão $c_{\mathrm{r}} / \mathrm{c}_{\mathrm{a}}$ também está ilustrada nesta figura, na escala da direita.

$O$ cálculo de $c_{a}$ e $c_{\mathrm{r}}$ resulta na aplicação das equações (4.19) e (4.20) para valores do ângulo de inclinação $\phi$ além do ponto de fissuração. Para cada ângulo $\phi$ da viga fissurada, a quantidade $z^{\prime}$ deve ser calculada, utilizando a equação (4.18) para obter a inércia efetiva $\mathrm{I}_{\mathrm{ef}}$. A curva do braço do momento resistente $c_{\mathrm{r}}$ é quase uma linha reta até $0,4 \operatorname{rad}(\operatorname{sen} \phi=0,97 \phi)$. A curva para o braço do momento aplicado $c_{a}$ tem um acréscimo em $\phi_{\text {máx }}$ devido à diminuição da rigidez, resultando no aumento de $z_{0}$.

$\mathrm{O}$ fator de segurança contra a ruptura pode ser obtido substituindo a inércia da seção fissurada pela inércia efetiva na equação (4.21), relativa ao fator de segurança contra fissuração. Assim, para $\mathrm{z}^{\prime}{ }_{0}=\mathrm{z}_{0}(1+2,5 \phi)$, tem-se:

$$
F S=\frac{c_{r}}{c_{a}}=\frac{y_{r} \phi}{z_{0} \phi(1+2,5 \phi)+e_{i}}
$$

O máximo ângulo de ruptura $\phi_{\text {máx }}^{\prime}$ pode ser calculado da seguinte forma:

$$
\frac{d F S}{d \phi}=0 \Rightarrow \phi_{\text {max de rupura }}=\phi_{\text {max }}^{\prime}
$$

Assim, a expressão do máximo ângulo de ruptura é dada por:

$$
\phi_{\text {max }}^{\prime}=\sqrt{\frac{e_{i}}{2,5 z_{0}}}
$$

onde o linha (') representa a ruptura e não a simbologia de derivada, conforme está esquematizado em (4.24). 


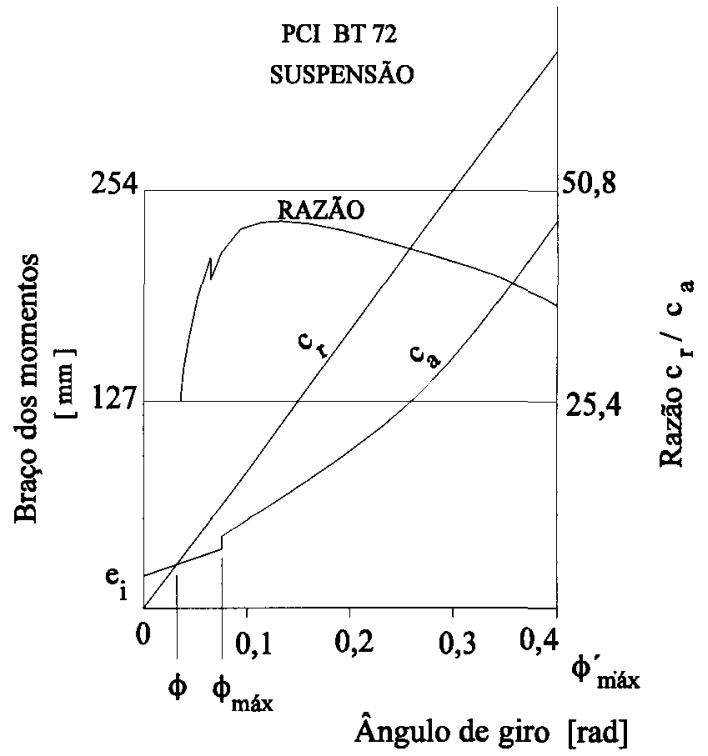

Figura 4.6 - Viga fissurada para $\phi>\phi_{\max \times}$ [ MAST (1993) ]

Substituindo na equação (4.23), os valores máximo ângulo de ruptura $\phi_{\text {máx }}^{\prime}$ e z ${ }_{0}^{\prime}$, resulta o fator de segurança contra ruptura FS':

$$
F S^{\prime}=\frac{y_{r} \phi_{\text {max }}^{\prime}}{z_{0}^{\prime} \phi_{\text {mix }}^{\prime}+e_{i}}
$$

Se o fator de segurança contra ruptura $\mathrm{FS}^{\prime}$ for menor que o fator de segurança contra fissuração, isto indica que o máximo fator de segurança ocorre justamente antes da fissuração. Neste caso, FS' deve ser igual a FS. 


\subsection{VIGAS PRÉ-MOLDADAS DE CONCRETO DURANTE O TRANSPORTE}

Quando a viga está apoiada sobre suporte flexível como almofadas de elastômero ou sobre os veículos de transporte, a tendência de giro da viga é em torno de um eixo na sua face inferior. O eixo de giro situa-se, portanto, abaixo do CG da viga.

A rigidez dos vínculos, nesta fase temporários, pode ser expressa como uma mola de constante elástica $\mathrm{K}_{\phi}$, representativa da somatória da rigidez dos elementos a serem considerados. Equacionando o equilíbrio dos momentos em torno do eixo de giro, conforme a figura 4.7, e fazendo aproximações para ângulos pequenos, tem-se:

$$
\begin{gathered}
P\left(\left(z_{0} \operatorname{sen} \phi+e_{i}\right) \cos \phi+y \operatorname{sen} \phi\right)=K_{\phi}(\phi-\alpha) \\
P\left(z_{0} \phi+e_{i}+y \phi\right)=K_{\phi}(\phi-\alpha)
\end{gathered}
$$

onde $\alpha$ é o ângulo de superelevação da pista de rolamento ou ângulo de inclinação do apoio, $\mathrm{z}_{0}$ é o deslocamento lateral do centro de massa do arco descrito pela viga fletida para todo o peso próprio aplicado lateralmente, $\mathrm{e}_{\mathrm{i}}$ é a excentricidade inicial do centro de gravidade da viga, e y é a altura do centro de gravidade da viga acima do eixo de giro.

Resolvendo a equação (4.27) para as aproximações assumidas, $\operatorname{sen} \phi=\phi$ e $\cos \phi=1$, e substituindo $\mathrm{r}$ por $\mathrm{K}_{\phi} / \mathrm{P}$, resulta:

$$
\phi=\frac{\alpha r+e_{i}}{r-y-z_{0}}
$$

Uma primeira análise da equação acima permite concluir que, quando r é muito grande, ou seja, o veículo é muito rígido, $\phi$ se aproxima de $\alpha$.

Da somatória dos momentos em torno do eixo de giro representado na figura 4.7, as equações para braço do momento aplicado $c_{a}$ e braço do momento resistente $c_{r}$ são:

$$
\begin{gathered}
c_{a}=\left(z_{0} \operatorname{sen} \phi+e_{i}\right) \cos \phi+y \operatorname{sen} \phi \\
c_{r}=r(\phi-\alpha)
\end{gathered}
$$

A equação (4.30) resulta da expressão do momento resistente igual a $\mathrm{K}_{\phi}(\phi-\alpha)$ e $\mathrm{r}$, dado por $\mathrm{K}_{\phi} / \mathrm{P}$. A diferença $\phi$ - $\alpha$ representa a rotação do mecanismo do veículo. 
Definindo como no item anterior, o fator de segurança equivale à relação entre $\mathrm{o}$ braço do momento resistente e o braço do momento aplicado. As aproximações para ângulos pequenos serão consideradas.

Para o máximo ângulo de inclinação $\phi_{\max }$ referente à fissuração, tem-se o fator de segurança FS contra fissuração:

$$
F S=\frac{r\left(\phi_{\max }-\alpha\right)}{z_{0} \phi_{\max }+e_{i}+y \Phi_{\max }}
$$

Os braços dos momentos ilustrados na figura 4.7 são muito maiores que para a mesma viga em suspensão ilustrada na figura 4.3. O momento aplicado é influenciado pelo alto valor de y que provoca o colapso em torno do eixo de giro. $\mathrm{O}$ valor fictício $\mathrm{z}_{0}$ não é tão importante quanto na viga em suspensão.

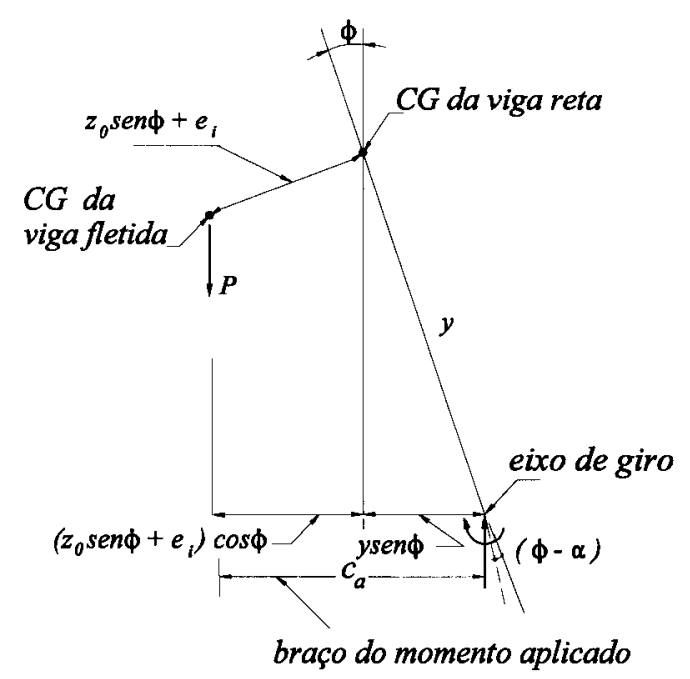

Figura 4.7 - Braço do momento aplicado para apoio na face inferior. [ MAST (1993) ]

MAST (1993), quanto à utilização da equação (4.31), apresenta informações positivas com a comprovação experimental de vigas que foram embarcadas e transportadas com sucesso: os fatores de segurança contra fissuração calculados para estas vigas resultaram muito próximo ou menor que 1.

Para definir o fator de segurança contra ruptura, é necessário conhecer o comportamento pós-fissuração da viga. Como feito para a viga em suspensão, o comportamento do elemento entre a fissuração e a ruptura pode ser descrito pelo 
diagrama das curvas dos braços dos momentos resistentes e aplicados.

A figura 4.8 mostra o diagrama de $c_{\mathrm{a}}$ e $c_{\mathrm{r}}$ para a viga modelo PCI BT-72. Nesta viga, a rotação referente ao equilíbrio em uma superelevação de $0,08 \mathrm{rad}$ ocorreu em torno de 0,113 radianos, sendo que o ângulo limite de fissuração era 0,10 rad.

A flexibilidade à rotação da carroceria do veículo possibilita rotações extras da viga, além da superelevação. Sugere-se o uso de equipamento para transporte bem rígido. Entretanto, uma pequena flexibilidade é necessária, não apenas para absorver impactos, mas também para prevenir excessivas tensões de torção, quando a cabine do caminhão e a carroceria estiverem sobre ângulos desiguais na pista de rolamento.

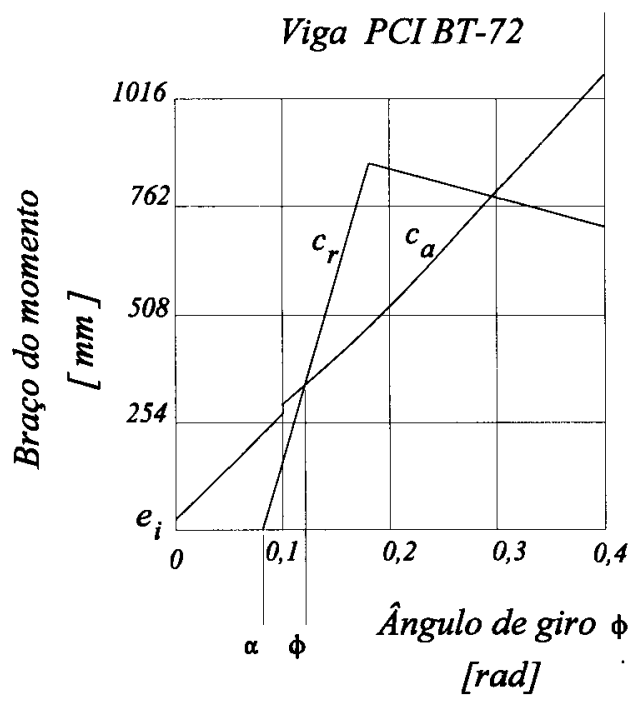

Figura 4.8 - Braço dos momentos x ângulo de inclinação da viga sobre a carroceria do caminhão. [ MAST (1993) ]

Os ensaios experimentais realizados por MAST (1993) indicaram que uma inclinação em excesso àquela que teoricamente provoca fissuração, pode ser tolerada. Além disso, nenhuma mudança no comportamento da viga, antes que o ângulo de inclinação fosse aproximadamente duas vezes o ângulo teórico de fissuração, foi observada.

$\mathrm{O}$ braço do momento resistente $\mathrm{c}_{\mathrm{r}}$ é limitado pela geometria do equipamento de transporte. Assumindo a altura $h_{r}$ em relação ao eixo de giro e a distância $z_{\text {máx }}$ do ponto 
central da carroceria até o centro entre as duas rodas do eixo traseiro de 4 pneus, o máximo braço do momento resistente $\mathrm{c}_{\text {rmáx }}$ pode ser encontrado como ilustrado na figura 4.9 .

$$
c_{r \max }=z_{\max } \cos \alpha-h_{r} \operatorname{sen} \alpha
$$

Substituindo a equação (4.30) na equação (4.32) e resolvendo para ângulo de giro $\phi_{\text {máx }}^{\prime}$, onde o braço do momento resistente é máximo, tem-se para as aproximações usuais:

$$
\phi_{\max }^{\prime}=\frac{z_{\operatorname{mix}}-h_{r} \alpha}{r}+\alpha
$$

Para encontrar o fator de segurança FS' contra ruptura, a equação (4.31) pode ser modificada pela substituição da seção fissurada, trocando $z_{0}$ por $z_{0}^{\prime}$ e $\phi_{\text {máx }}$ por $\phi_{\text {máx }}^{\prime}$.

$$
F S^{\prime}=\frac{r\left(\phi_{\max }^{\prime}-\alpha\right)}{z_{0}^{\prime} \phi_{\max }^{\prime}+e_{i}+y \phi_{\max }^{\prime}}
$$

Quanto à importância da rigidez da mola $\mathrm{K}_{\phi}$, pode-se visualizar o seu efeito nos fatores de segurança contra fissuração e ruptura através da figura 4.10.

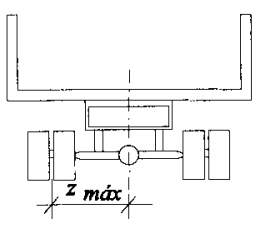

VISTA TRANSVERSAL

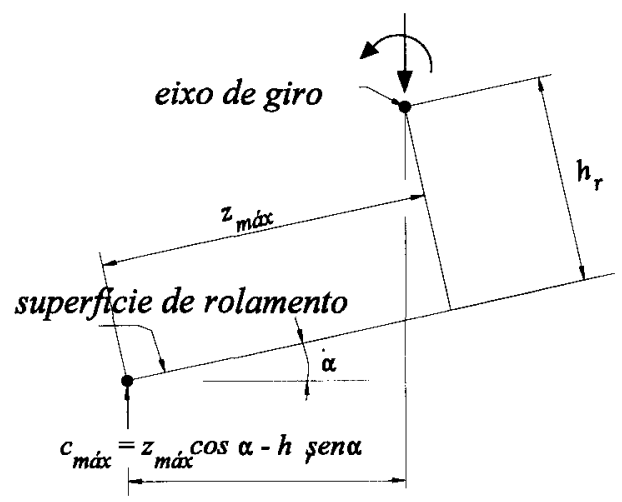

Figura 4.9 - Máximo braço do momento resistente durante o transporte. [ MAST (1993) ]

A rigidez ao giro dos veículos de transporte, dada pela constante de mola à rotação, é um parâmetro importante na avaliação da segurança de vigas esbeltas durante 
o transporte. Como informações sobre a rigidez ao giro dos veículos não são comuns, a rigidez pode ser determinada de forma simples e muito válida, pois será obtida no próprio veículo, pela disposição de pesos em vários pontos excêntricos à linha de centro.

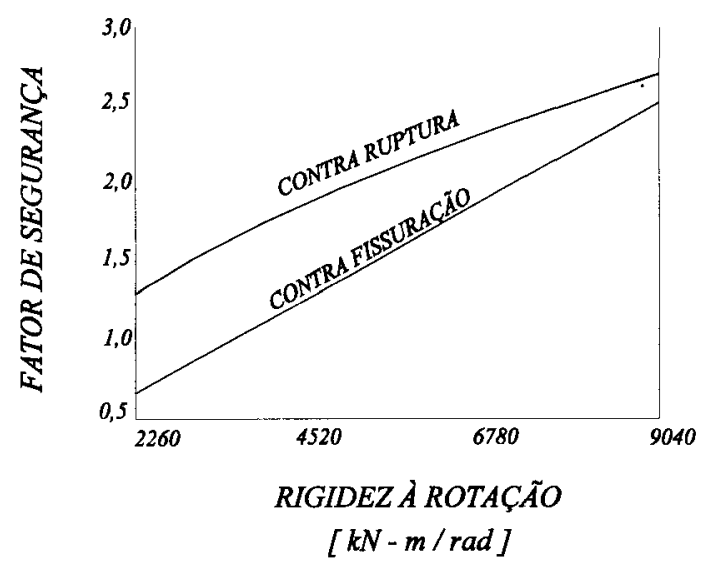

Figura 4.10 - Importância da rigidez à rotação da carroceria e da cabine. [ MAST (1993) ]

MAST (1993) recomenda um método simples para medir a rigidez ao giro: com o equipamento sobre uma superfície plana ou nivelada, mede-se o ângulo de inclinação da viga nos dois apoios (eixo dianteiro e eixo traseiro) e calcula a média. Depois, com o veículo estacionado sobre o pavimento com uma certa inclinação, mede-se o ângulo de inclinação da viga nos dois apoios e calcula-se a média. $O$ valor de $\phi$ deve ser definido como a diferença entre as leituras iniciais e as finais. Então, a estimativa de $\mathrm{r}=\mathrm{K}_{\phi} / \mathrm{P}$ resulta $\mathrm{em}:$

$$
r=\frac{\left(y+z_{0}\right) \phi}{\phi-\alpha}
$$

CUNHA (1994) apresenta um processo simples para a determinação experimental da rigidez à rotação da carroceria do caminhão a ser utilizado no transporte das vigas, a partir da utilização de recursos existentes na própria fábrica. Dois ensaios foram realizados: no primeiro considerou-se a carroceria na posição normal, conforme a figura 4.11 e no outro, a carroceria na posição extendida, como na figura 4.12. Em ambos os ensaios, foram utilizadas quatro vigas pré-moldadas de concreto, das quais eram conhecidos o peso $\mathrm{e}$ as dimensões. Estas vigas foram posicionadas 
sistematicamente sobre a carroceria do caminhão. A rigidez à rotação da carroceria foi obtida a partir da leitura da rotação da mesma, para os pesos conhecidos. O valor da constante de mola à rotação é dado pelo momento aplicado em relação à respectiva rotação.

No estudo experimental de CUNHA (1994), para o caminhão na posição normal, os valores obtidos para a rigidez apresentaram uma certa uniformidade. Entretanto, são valores representativos apenas da rigidez da parte posterior situada sobre os eixos traseiros da carroceria. $O$ cálculo da rigidez da cabine pode ser dispensado se a rigidez medida para os eixos traseiros proporcionar estabilidade às vigas durante o transporte, pois a rigidez da cabine do caminhão só irá contribuir positivamente para aumentar a rigidez do conjunto.

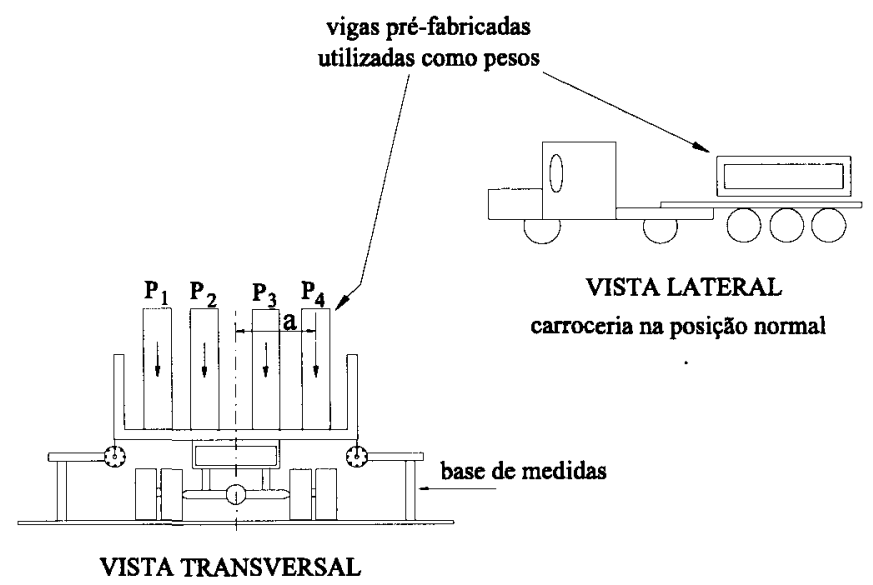

Figura 4.11 - Situação das cargas no caso da carroceria em posição normal.

[ CUNHA (1994)]

Para os ensaios experimentais com a carroceria do caminhão na posição extendida, que representa a situação real em que se pretende fazer o transporte das vigas, CUNHA (1994) verifica que quando o caminhão encontra-se mais carregado, ocorre um pequeno aumento do valor médio do coeficiente $\mathrm{K}_{\phi}$. 


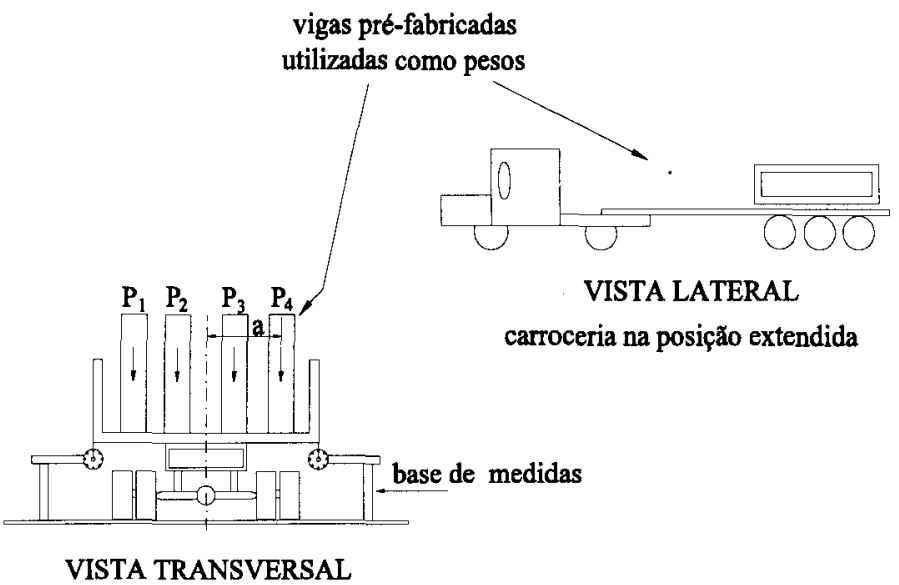

Figura 4.12 - Situação das cargas para carroceria em posição extendida. [ CUNHA (1994) ]

Quanto à determinação da superelevação da pista de rolamento, CUNHA (1994) recomenda um dispositivo de ensaio muito simples, composto de uma mangueira de nível e duas réguas graduadas em centímetros. Faz-se a disposição destes instrumentos sobre a carroceria do próprio caminhão no eixo central posterior, conforme representado na figura 4.12. As leituras devem ser feitas com o veículo parado sobre diversos pontos críticos escolhidos na trajetória. Este procedimento é considerado viável porque é realizado no próprio caminhão e inclui as características do trajeto a ser percorrido.

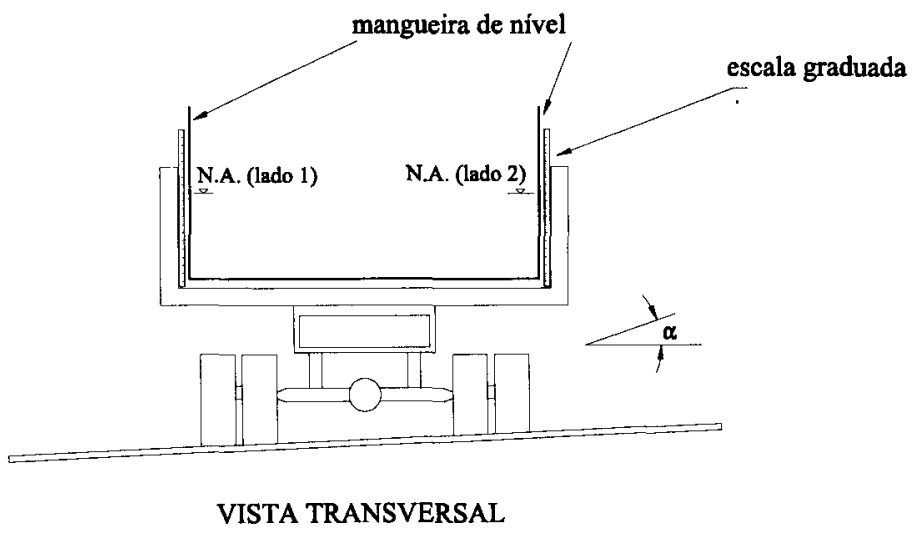

Figura 4.13 - Esquema para a determinação da superelevação da estrada medida no próprio caminhão de transporte. [ CUNHA (1994)] 


\subsection{CONSIDERAÇÕES GERAIS SOBRE OS FATORES DE SEGURANÇA}

Alguns comentários em relação à definição do fator de segurança necessário devem ser feitos. Um fator de segurança, além do embasamento matemático e das leis científicas, deve ser determinado e comprovado experimentalmente.

O PCI Design Handbook $3^{a}$ edição indica a utilização de um fator de segurança igual a 2 para a fase de suspensão. Em 1963, alguns ensaios com vigas protendidas de aproximadamente $45 \mathrm{~m}$ de comprimento, indicaram que um fator igual a 2 era adequado quando a excentricidade inicial em relação às tolerâncias fosse muito pequena.

IMPER \& LASZLO (1987) sugerem o uso de um fator de 1,5 para manuseio no canteiro da fábrica e 1,75 para manuseio no local da edificação, ambos relativos à fase de suspensão. MAST (1993) recomenta utilizar um fator de segurança igual a 1 contra fissuração e igual a 1,5 contra ruptura, para vigas em suspensão e para as vigas apoiadas na face inferior, como ocorre durante o transporte.

O efeito benéfico dos balanços na fase transitória deve ser sempre que possível aproveitado. A figura 4.14 ilustrada em MAST (1993) mostra como os fatores de segurança aumentam quando a suspensão é realizada com balanços. Para o critério de verificação da resistência utilizada no PCI, o melhor comprimento para os balanços variam entre 2,1 a $2,7 \mathrm{~m}$ para a viga protendida PCI BT-72.

IMPER \& LASZLO (1987), no gráfico esquematizado na figura 4.15, mostram como a presença dos balanços influenciam a carga crítica, bem como o fator de segurança. $O$ posicionamento dos cabos de suspensão a partir da extremidade da viga, pode aumentar significativamente a estabilidade desta fase, reduzindo a flecha no meio de vão, bem como o deslocamento $z_{0}$. 


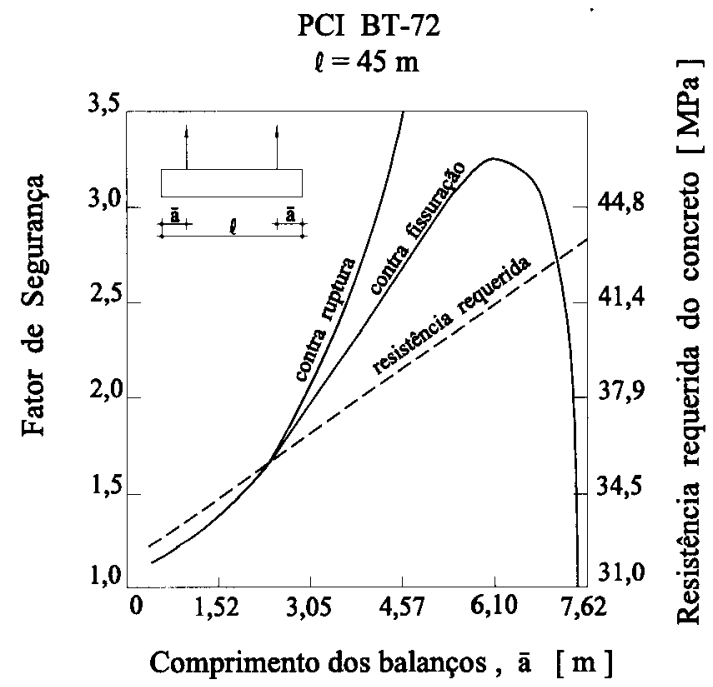

Figura 4.14 - Efeito dos balanços no fator de segurança em vigas na fase de suspensão. [ MAST (1993) ]

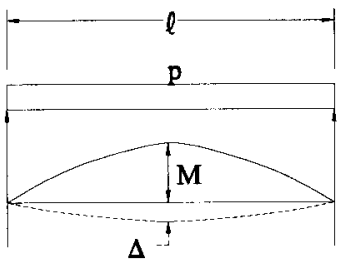

$\mathrm{M}=\frac{\mathrm{p} \ell^{2}}{8} \quad \Delta=\frac{5 \mathrm{p} \ell^{2}}{384 \mathrm{EI}}$

(a)

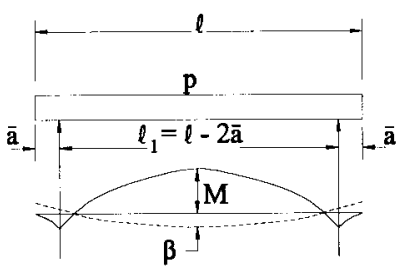

$M=p\left(\ell_{1}-4 \bar{a}^{2}\right)$

8

$\beta=p \ell_{1}\left(5 \ell_{1}^{2}-24 \bar{a}\right)$

384EI

(b)

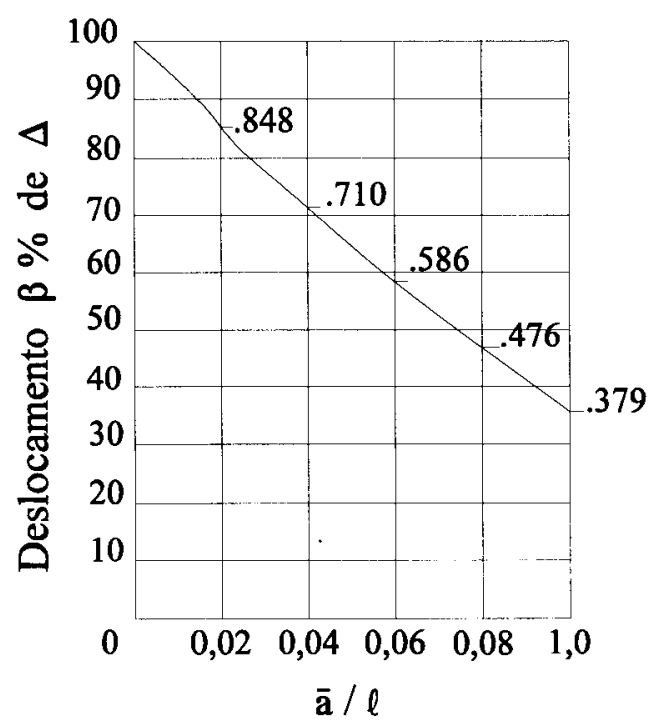

(c)

EXEMPLO:

$30,50 \mathrm{~m}$ de viga simplesmente apoiada: $\Delta=2,54 \mathrm{~cm}$ mesma viga apoiada com balanços de $15,25 \mathrm{~cm}$ à partir das extremidades: $\beta=1,47 \mathrm{~cm}$

Figura 4.15 - Influência da posição dos balanços em vigas protendidas. [ IMPER \& LASZLO (1987)] ] 
A figura $4.15 \mathrm{c}$, para a relação $\bar{a} / \ell$ fornece a porcentagem da flecha máxima da viga com balanços em relação à disposição sem balanços, onde à é o comprimento do balanço e $\ell$ é o vão total. Por exemplo, para um balanço equivalente a $6 \%$ do vão, a flecha máxima diminue para $58,8 \%$ da flecha para a mesma viga apoiada nas extremidades. Além disso, o valor de $\mathrm{z}_{0}$ cai para a metade, o que duplica o fator de segurança.

O mínimo fator de segurança aplicado aos cálculos não considera os efeitos de deformação lenta e impacto, os quais são preferencialmente importantes durante o transporte em veículo, especialmente quando este passa sobre uma superelevação em baixa velocidade. IMPER \& LASZLO (1987) recomendam considerar separadamente os efeitos de impacto e superelevação, em razão da pouca probabilidade de ocorrência da intensidade máxima destes efeitos simultaneamente. A suspensão e as superelevações das rodovias são atividades passageiras para que se preocupe com os problemas de fluência. Entretanto, é recomendável que a viga não seja mantida em posição inclinada por um tempo suficientemente capaz de tornar os efeitos de deformação lenta significativos.

Nas vigas em suspensão, como o peso e a rigidez são conhecidos, uma das incógnitas é a excentricidade inicial devido às imperfeições construtivas e aos desvios de posicionamento dos cabos. Medindo o ângulo $\phi$ de inclinação da viga durante o içamento, o fabricante pode utilizar a equação (4.9), fazendo $\operatorname{tg} \phi \sim \phi$, para determinar o valor da excentricidade inicial e ajustar os cálculos.

$$
e_{i}=\left(y_{r}-z_{0}\right) \phi
$$

A verificação da segurança requer o conhecimento de $\mathrm{e}_{\mathrm{i}} \mathrm{e} \phi_{\operatorname{máx}}$. A excentricidade inicial pode ser obtida como citado acima ou através de uma combinação crítica das tolerâncias. O PCI Design Handbook $3^{\mathrm{a}}$ edição faz algumas indicações práticas para o cálculo desta excentricidade, as quais serão citadas no capítulo 5. A determinação de $\phi_{\operatorname{máx}}$ envolve algumas dificuldades. Usando um programa para avaliação da resistência limite por flexão nos dois planos, o máximo momento que produz flexão lateral em combinação com a flexão vertical pode ser encontrado, resultando então, $\phi_{\operatorname{máx}}$ para a 
carga crítica. Entretanto, uma vez que o momento lateral excede a resistência à fissuração, a rigidez diminue e $z_{0}$ aumenta. Uma aproximação conservativa é calcular $\phi_{\text {máx }}$ baseado no momento lateral que, quando combinado com o momento vertical, produz uma tensão nas fibras superiores igual à tensão última. $O$ valor correto de $\phi_{\operatorname{máx}}$ provavelmente está entre esta aproximação e a da resistência última.

O cuidado de tentar aumentar a estabilidade da viga durante a suspensão resulta em algumas recomendações, as quais encontram-se também descritas no estudo de MAST (1989), e que serão detalhadas a seguir.

Mover os pontos de suspensão a partir da extremidade da viga é um dos mais efetivos procedimentos que aumentam a estabilidade lateral do elemento estrutural durante a suspensão. A segurança chega a duplicar para um balanço equivalente a $6 \%$ do vão, conforme já definido. Entretanto, as tensões nas seções críticas precisam ser conferidas. IMPER \& LASZLO (1987) sugerem o uso temporário da pós-tensão no topo da mesa superior, onde é necessário controlar as tensões.

Porém, diminuir o vão de suspensão e transporte, pode em algumas situações não ser viável, porque em fase de projeto a viga é dimensionada para carregamento e tipo de vinculação relativos à disposição definitiva. É difícil, em alguns casos, mover os pontos de suspensão a partir da extremidade, sem aumentar significativamente as tensões na seção do meio do vão. Isso ocorre especialmente em vigas protendidas, porque quando suspensa com balanços, o momento fletor na seção central devido ao peso-próprio é menor que no içamento pelas extremidades. Logo, as tensões finais são maiores, já que o alívio de tensões devido ao peso-próprio frente à protensão é menor.

Outro meio é levantar o eixo de giro acima da superfície superior da viga. Isto pode ser feito usando uma barra rígida fixa à viga no ponto de suspensão, de forma que os cabos de içamento sejam presos nesta barra.

Aumentar o módulo E de elasticidade, resulta no aumento da resistência do concreto. Estes acréscimos melhoram a capacidade da viga de suportar as tensões atuantes. Por outro lado, aumentar o módulo de elasticidade do concreto e a resistência à compressão apenas para suportar a fase transitória de suspensão muitas vezes é inviável economicamente. $\mathrm{O}$ aumento desses valores, dentro de um certo limite é 
aceitável, porque geralmente as tensões nas seções são mais críticas na fase transitória do que na fase em serviço, no caso das vigas protendidas. Nesta fase, não ocorre o carregamento acidental que promove a redução das tensões devido à protensão.

A disposição de contraventamentos é utilizada, apesar de ser um dos meios menos efetivos, caso o contraventamento não apresente substancial área de aço. Porém, podem aumentar significativamente a resistência à flexão lateral, e portanto, $\phi_{\text {máx }}$. Este procedimento é de certa forma seguro quando altos valores de $\phi$ são obtidos, como por exemplo, em curvas superelevadas.

O uso de contraventamento tem o seu mérito e era muito utilizado para manuseio e transporte de vigas no passado. No entanto, se toda viga tiver que ser enrijecida, este tipo temporário de contraventamento pode ser muito caro, e além de um determinado valor do deslocamento lateral, o sistema geralmente é inútil e não previne o colapso da viga.

O sistema de contraventamento pode ser mais efetivo quando a viga está sendo transportada em caminhão, quando este se movimenta sobre as superelevações. Como as deformações não são instantâneas, o movimento compensa as inclinações da via de tráfego, visto que estas são passageiras. Mas a situação é crítica quando o veículo de transporte pára sobre uma determinada superelevação ou superfície irregular, porque as deformações horizontais não podem ser ajustadas ou interrompidas.

IMPER \& LASZLO (1987) citam o exemplo de uma viga que estava sendo içada do caminhão e teve que ser recolocada na pista de rolamento porque o guindaste não suportava o peso da mesma. Mas devido à superelevação da via onde foi posicionada, a viga apresentou $8,67^{\circ}$ de inclinação lateral. Após 20 minutos, atingiu o deslocamento crítico. A viga em questão era de seção duplo T com 2,13m de altura e $40 \mathrm{~m}$ de extensão.

A alteração da seção transversal não é um meio comumente utilizado em projetos, com o objetivo de aumentar a estabilidade das fases transitórias. Mas, quando a seção de uma viga pode ser alterada, é importante que apresente uma mesa inferior que contribua ao máximo para a estabilidade, tanto quanto a mesa superior. De fato, adicionar material à mesa inferior é benéfico porque abaixa o $C G$, aumenta $\mathrm{y}_{\mathrm{r}} \mathrm{e} \mathrm{I}_{\mathrm{y}}$. Além 
do mais, a mesa inferior, no caso de uma viga protendida, está submetida a compressão e não deve perder rigidez por fissuração como pode ocorrer com a mesa superior. 


\section{EXEMPLOS NUMÉRICOS E ANÁLISES DOS RESULTADOS}

\subsection{PRELIMINARES}

Serão analisados neste capítulo alguns exemplos numéricos das formulações tratadas nos capítulos precedentes. Maior ênfase será dada à fase transitória de suspensão. Algumas comparações com respeito à maior estabilidade da fase de serviço em relação ao içamento por cabos, bem como indicações de uso e análises críticas serão detalhadas para cada formulação. O quadro 5.1 mostra os casos analisados.

$\mathrm{Na}$ fase de serviço, as vigas estão apoiadas sobre vínculos de garfo nas extremidades, cuja deformabilidade será avaliada para a viga de seção retangular. A suspensão é definida por cabos acoplados à viga em ganchos previamente fixados na face superior.

A viga retangular de concreto pré-moldado, nas duas fases de análise, será avaliada pela formulação por séries de potência e pelo programa desenvolvido neste trabalho, utilizando o método numérico de Runge-Kutta.

A viga de seção duplo T simétrico de argamassa armada será analisada em serviço quanto à carga crítica de flambagem lateral, pela expressão apresentada em 
Quadro 5.1 - Esquema geral dos casos analisados e os respectivos processos de cálculo.

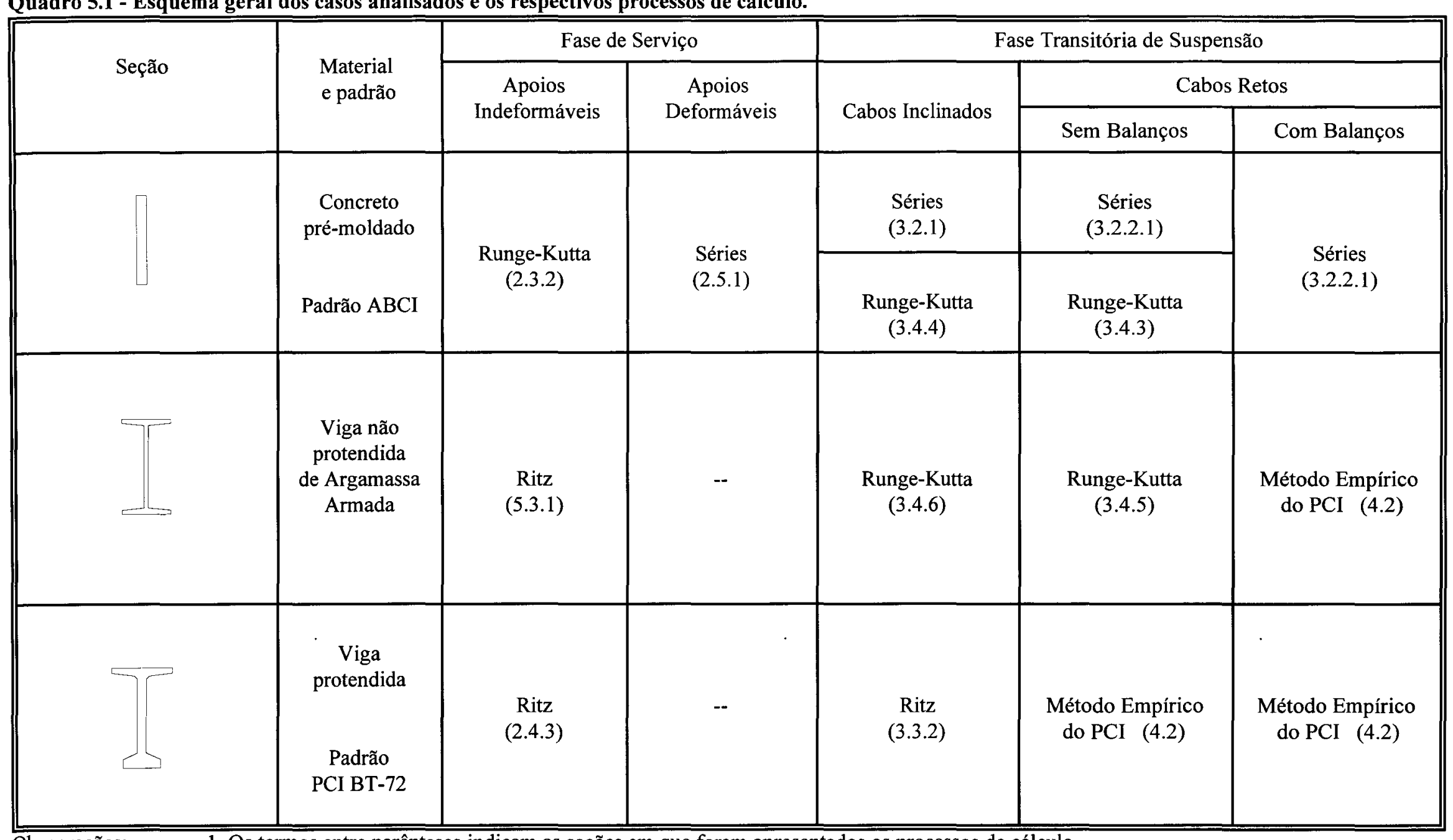


RACHID (1993). Na fase transitória de suspensão, o estudo numérico será desenvolvido a partir dos resultados dos programas (Runge-Kutta) e pela adaptação do trabalho de MAST (1993).

A viga protendida será analisada pelos estudos empíricos de IMPER \& LASZLO (1987) e MAST $(1989,1993)$. O trabalho analítico de CATANIA \& COCCHI (1985) desenvolvido pelo método da energia potencial, na aplicação do processo de Ritz, complementa as avaliações comparativas.

Uma indicação prática, sem maiores comentários, encontra-se em KONCZ (1978), o qual considera segura toda a elevação cuja carga crítica de instabilidade seja superior a 4 vezes o peso próprio. Este fator inclui além da segurança, uma parcela referente às imperfeições construtivas.

A norma brasileira NBR-9062/85 indica o uso de um coeficiente de ação dinâmica, quando uma análise específica não puder ser efetuada, durante a montagem e a fase de manuseio no canteiro. Neste caso, a solicitação pode ser considerada, por uma solicitação estática equivalente, através do coeficiente 1,3. O CEB MC/90 recomenda, na falta de determinação mais acurada, que os efeitos verticais dinâmicos do levantamento e transporte podem ser levados em conta pela consideração do peso próprio com um fator $1 \pm 0,2$.

Neste trabalho, será adotado o fator 4 indicado em KONCZ (1978), com o objetivo de considerar as possíveis imperfeições construtivas, e o coeficiente 1,3, para as ações dinâmicas na fase transitória.

As análises da instabilidade lateral, nas hipóteses de regime elástico linear, serão desenvolvidas através da relação carga crítica em relação ao peso próprio. Esta relação, representada por RCP será comparada ao fator $1,3 \times 4$, ou 5,20, devendo ser maior ou igual a este valor. Para este limite será verificada a segurança do elemento prémoldado quanto aos problemas de perda de estabilidade lateral durante a fase transitória.

\subsection{VIGA DE SEÇÃO RETANGULAR}

A viga de seção retangular a ser analisada é um modelo padronizado pela 
Associação Brasileira da Construção Industrializada, ABCI (1986), denominada vigatesteira ou platibanda. A figura 5.1 ilustra a seção transversal da viga em estudo, cujas relações altura/vão e altura/espessura permitem, numa aproximação, assumir a seção como delgada.

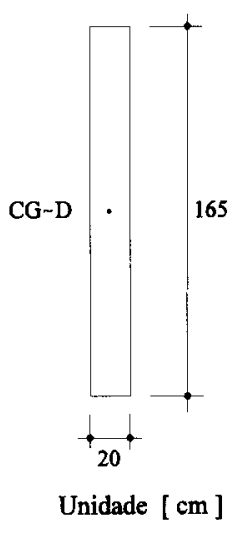

Figura 5.1 - Seção transversal padrão da ABCI .
O módulo de elasticidade longitudinal E é $3460 \mathrm{kN} / \mathrm{cm}^{2}$ e o módulo transversal $\mathrm{G}$ é igual a 1440 $\mathrm{kN} / \mathrm{cm}^{2}$.

A viga-testeira tem $20 \mathrm{~m}$ de comprimento e a área da seção transversal é $3300 \mathrm{~cm}^{2}$.

$\mathrm{O}$ peso próprio da viga de concreto armado equivale a 0,0825 $\mathrm{kN} / \mathrm{cm}$.

As características geométricas da seção transversal da viga em análise são:

$$
\begin{array}{ll}
I_{y}=110000 \mathrm{~cm}^{4} & I_{z}=7486875 \mathrm{~cm}^{4} \\
J_{t}=440000 \mathrm{~cm}^{4} & i_{D}{ }^{2}=2302,08 \mathrm{~cm}^{2}
\end{array}
$$

\subsubsection{FASE DE SERVIÇO}

\subsubsection{Apoios Indeformáveis}

Com a expressão (2.36) da carga crítica, obtida pela resolução da equação diferencial regente da instabilidade lateral através do método numérico de Runge-Kutta, tem-se: 


$$
\begin{aligned}
& p_{c r i t}=28,31 \frac{\sqrt{G J_{t} E I_{y}}}{\ell^{3}} \\
& p_{\text {crit }}=28,31 \frac{\sqrt{633,6 \cdot 10^{6} \cdot 380,6 \cdot 10^{6}}}{(2000)^{3}}=1,74 \frac{\mathrm{kN}}{\mathrm{cm}} \\
& R C P=\frac{1,74}{0,0825}=21,09
\end{aligned}
$$

Na relação RCP igual a 21,09, equivalente a quantas vezes a carga crítica de instabilidade lateral supera o peso próprio, verifica-se durante a fase de serviço a grande segurança com relação à estabilidade lateral apresentada pela viga apoiada sobre vínculos rígidos à torção.

\subsubsection{Apoios Deformáveis}

A carga crítica da viga-testeira apoiada sobre vínculos deformáveis à torção depende da constante elástica $\mathrm{K}_{\phi}$, que representa a rigidez da ligação.

Na ligação de garfo deformável à torção, ilustrada na figura 5.2, a inércia em relação ao giro lateral, o módulo de elasticidade e a rotação produzida por momento unitário, permitem de forma aproximada, estimar numericamente a rigidez à torção do vínculo. Lembrar que este cálculo é grosseiro e não é objetivo deste trabalho.

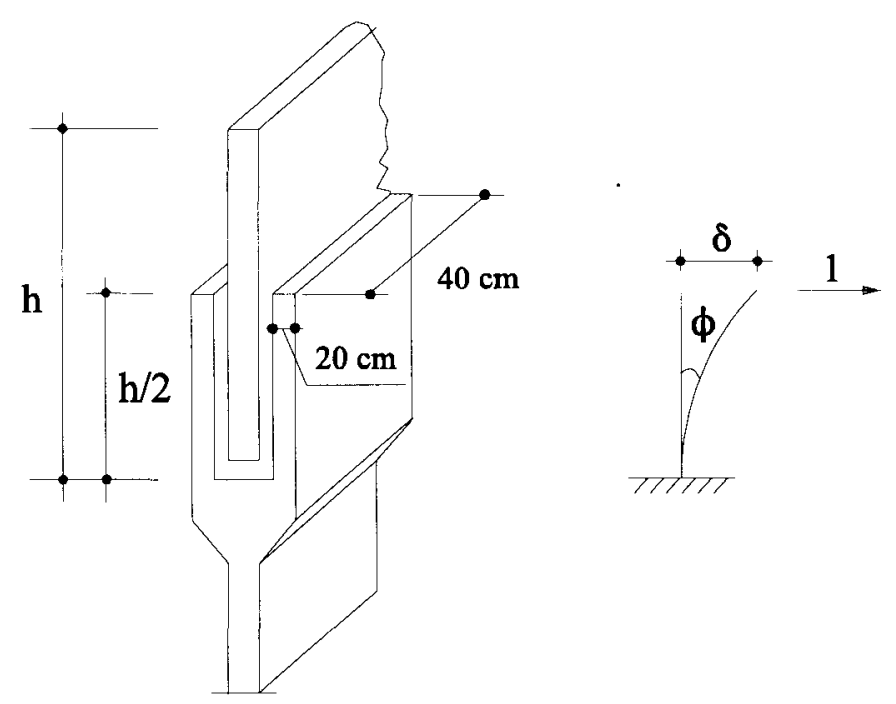

Figura 5.2 - Ligação de garfo 
Com base nestas hipóteses a rigidez da ligação será assumida igual a $2,9091 \times 10^{6} \mathrm{kNcm}$.

No cálculo da carga crítica pelo estudo utilizando séries de potência, tem-se:

a) $f(\alpha)=\frac{\ell}{2} \frac{K_{\phi}}{G J_{t}}=\frac{2000}{2} \frac{2,9091 \cdot 10^{6}}{633,6 \cdot 10^{6}}=4,5914$

b) Para $f(\alpha)=4,5914$

$\therefore$ na equação (2.91) ou no gráfico da figura $2.8: \psi=16 \sqrt{\alpha}=24,4496$

c) $p_{\text {crit }}=\frac{24,4496 \sqrt{380,6 \cdot 10^{6} \cdot 633,6 \cdot 10^{6}}}{(2000)^{3}}=1,50 \mathrm{KN} / \mathrm{cm}$

d) $R C P=\frac{1,50}{0,0825}=18,19$

O valor da carga crítica para a viga de seção retangular apoiada nas extremidades por vínculos de garfo, cuja deformabilidade à torção permite giro e deslocamentos laterais nos apoios, é menor que o valor resultante da ligação rígida. Para ligações com alta deformabilidade à torção, a carga crítica na fase de serviço atinge valores mais críticos. Mas, em relação à fase transitória de suspensão, a carga crítica avaliada neste item é ainda muito superior.

\subsubsection{FASE TRANSITÓRIA DE SUSPENSÃO}

\subsubsection{Cabos Inclinados}

$\mathrm{Na}$ disposição dos cabos de içamento inclinados fixos às seções extremas, o estudo da carga crítica será desenvolvido utilizando a formulação da instabilidade do trabalho de CATANIA \& COCCHI (1985), onde aplica-se o método de integração por séries de potência e estudo desenvolvido neste trabalho, a partir da teoria da instabilidade em seções delgadas, apresentada em RACHID (1993), no qual o método 
numérico de Runge-Kutta foi utilizado para a resolução das equações diferenciais. É importante lembrar, que não estão sendo comparados os processos de resolução de expressões diferenciais, mas sim, a formulação do estudo da instabilidade lateral.

\subsection{Séries de potência}

O esquema da suspensão com cabos inclinados considera os cabos presos às extremidades da viga, como ilustrado na figura 3.17. Desta forma, os ângulos definidos no estudo de CATANIA \& COCCHI (1985) são: $\beta=0^{\circ}$ e $\gamma$ variável.

Entretanto, para estes valores de $\beta$ e $\gamma$, o resultado da distância $e$ resulta sempre o mesmo e igual à distância $\mathrm{d}$ do centro de gravidade à face superior da viga, conforme a figura 3.1. Nestes termos, tem-se:

$$
\begin{gathered}
\beta=0^{\circ} \quad \text { e } \quad \gamma=\text { qualquer } \\
e=d=82,5 \mathrm{~cm}
\end{gathered}
$$

a) $f(\alpha)=\frac{\ell}{2} \frac{R_{x}}{G J_{t}}=\frac{2000}{2} \frac{35392,50}{633,6 \cdot 10^{6}}=0,0559$ onde $R_{x}=\frac{1}{2} p l e$

b) Para $f(\alpha)=0,0559$

$\therefore \quad$ na expressão (3.11) ou na figura 3.3: $\psi=16 \sqrt{\alpha}=5,0954$

c) $p_{\text {crit }}=\frac{5,0954 \sqrt{380,610^{6} \cdot 633,6 \cdot 10^{6}}}{(2000)^{3}}=0,313 \mathrm{KN} / \mathrm{cm}$

d) $R C P=\frac{0,313}{0,0825}=3,79<5,20$

Para as limitações desta formulação, onde a variação da inclinação do cabo de suspensão não altera o valor da carga crítica de instabilidade lateral, equivale dizer que, a força normal oriunda da decomposição da força de tração dos cabos na direção horizontal não é considerada. Apesar desta inconsistência, a inclinação $\gamma$ não introduz acréscimos tão significativos na carga crítica, como se verifica nos resultados obtidos dos programas elaborados neste trabalho. 
Da relação RCP menor que o valor adotado para a segurança da fase transitória, tem-se uma situação com grandes riscos de instabilidade lateral por mudança de equilíbrio.

\subsection{Runge-Kutta}

Para várias inclinações dos cabos, a tabela 5.1 ilustra os valores da carga crítica da viga de seção retangular.

Tabela 5.1 - Ângulos $\gamma \times$ carga crítica

Viga-testeira padrão da ABCI

\begin{tabular}{|c|c|}
\hline$\gamma[$ graus $]$ & $p_{\text {crit }}[\mathrm{kN} / \mathrm{cm}]$ \\
\hline $50^{\circ}$ & 0,193 \\
\hline $60^{\circ}$ & 0,204 \\
\hline $70^{\circ}$ & 0,213 \\
\hline $80^{\circ}$ & 0,223 \\
\hline $90^{\circ}$ & 0,233 \\
\hline $100^{\circ}$ & 0,243 \\
\hline $110^{\circ}$ & 0,256 \\
\hline $120^{\circ}$ & 0,271 \\
\hline $130^{\circ}$ & 0,293 \\
\hline
\end{tabular}

A relação $\mathrm{RCP}$, para os ângulos de inclinação definidos na tabela 5.1, varia entre 2,30 e 3,55, sendo ainda menor que 5,20 para qualquer um dos ângulos de içamento.

Nota-se a diferença entre os resultados da carga crítica obtidos do programa desenvolvido neste trabalho em relação ao estudo apresentado por CATANIA \& COCCHI (1985), mostrado no item anterior, para a inclinação $\gamma$ igual a $90^{\circ}$. 


\subsubsection{Cabos Retos}

\subsection{Sem balanços}

A carga crítica de suspensão por cabos retos presos às extremidades, obtida do estudo da instabilidade pela aplicação do método de Runge-Kutta, conforme a tabela 5.1, é $0,233 \mathrm{kN} / \mathrm{cm}$.

Por séries, no estudo da suspensão por cabos retos sem balanços, o valor adimensional de $\overline{\mathrm{d}}$ deve ser igual a 1. Para $\beta$ tendendo a zero e $\gamma$ igual a $90^{\circ}$, resulta a excentricidade $e$, equivalente à distância do centro de gravidade à face superior da viga, onde localizam-se os pontos de fixação dos cabos de içamento.

$$
\begin{gathered}
\bar{d}=1 \\
\beta \sim 0^{\circ} \text { e } \gamma=90^{\circ} \\
e=d=82,5 \mathrm{~cm}
\end{gathered}
$$

a) $j(\alpha, \bar{d})=2 \frac{e}{a} \sqrt{\frac{E I_{y}}{G J_{t}}}=2 \frac{82,5}{1000} \sqrt{\frac{380,6 \cdot 10^{6}}{633,6 \cdot 10^{6}}}=0,1279$

b) Para $j(\alpha, 1)=0,1279 \quad \therefore$ na figura 3.5: $\alpha=0,0558$

c) $p_{\text {crit }}=\frac{16 \sqrt{0,0558} \sqrt{380,6 \cdot 10^{6} \cdot 633,6 \cdot 10^{6}}}{(2000)^{3}}=0,232 \mathrm{KN} / \mathrm{cm}$

d) $R C P=\frac{0,232}{0,0825}=2,81$

O estudo de CATANIA \& COCCHI (1985), relativo à suspensão por cabos retos, apresenta resultados praticamente coincidentes com os obtidos do programa para cálculo da carga crítica de instabilidade lateral, através do método de Runge-Kutta.

Pela verificação das tensões, a seção apresenta-se fissurada. Além disso, observa-se pela relação RCP inferior a 5,20, a ocorrência muito provável da flambagem lateral. 


\subsection{Com balanços}

Este tipo de suspensão é geralmente eficaz porque aumenta de forma considerável o valor da carga crítica. Os resultados obtidos a partir da formulação de CATANIA \& COCCHI (1985) estão dispostos na tabela 5.2.

$$
p_{\text {crt }}=\frac{16 \sqrt{\alpha} \sqrt{380,6 \cdot 10^{6} \cdot 633,6 \cdot 10^{6}}}{(2000)^{3}}
$$

Tabela 5.2 - Valores da carga crítica conforme variação de đ.

\begin{tabular}{|c|c|c|c||}
\hline $\mathrm{d}$ & $\alpha$ & $\mathrm{p}_{\text {crit }}[\mathrm{kN} / \mathrm{cm}]$ & Observações \\
\hline 1,0 & 0,05584 & 0,232 & Suspensão sem balanços \\
\hline 0,8 & 0,68275 & 0,812 & $\dot{A} 0,1 \ell$ das extremidades \\
\hline 0,6 & 90,55163 & 9,346 & $\grave{A} 0,2 \ell$ das extremidades \\
\hline 0,55 & 255,05871 & 15,685 & $\dot{\mathrm{A}} 0,225 \ell$ das extremidades \\
\hline 0,5 & 96,62948 & 9,654 & $\mathrm{Em} \mathrm{x}=1 / 4 \ell \mathrm{e} \mathrm{x}=3 / 4 \ell$ \\
\hline 0,3 & 2,01843 & 1,395 & $\dot{\mathrm{A}} 0,35 \ell$ das extremidades \\
\hline 0,0 & 0,40285 & 0,623 & Suspensão única em $\mathrm{x}=\ell / 2$ \\
\hline
\end{tabular}

A presença dos balanços incrementa consideravelmente a carga crítica de instabilidade lateral, além de beneficiar a integridade do elemento por apresentar menores tensões de tração.

Os valores de $\alpha$ foram obtidos a partir do desenvolvimento matemático da expressão (3.38). As figuras 3.5 à 3.8 representam os gráficos da função $\mathrm{j}(\alpha, \overline{\mathrm{d}})$. Como definido na teoria do capítulo 3, para d igual a 0,55 a carga crítica é em torno de 65 vezes maior que a carga crítica na suspensão pelos extremos da viga.

Cabe observar que o cálculo da carga crítica apresentado neste trabalho assume as hipóteses de regime elástico-linear. A viga-testeira de $20 \mathrm{~m}$ de comprimento mostra-se fissurada para a mais estável disposição de balanços. Portanto, encontra-se fora das condições estabelecidas para o estudo da instabilidade elástica. Maiores comentários serão definidos no capítulo 6 . 


\subsection{VIGA DE ARGAMASSA ARMADA}

A viga de argamassa armada não protendida com $15 \mathrm{~m}$ de comprimento e seção duplo $\mathrm{T}$ simétrico está ilustrada na figura 5.3. A seção transversal apresenta as espessuras típicas do material constituinte.

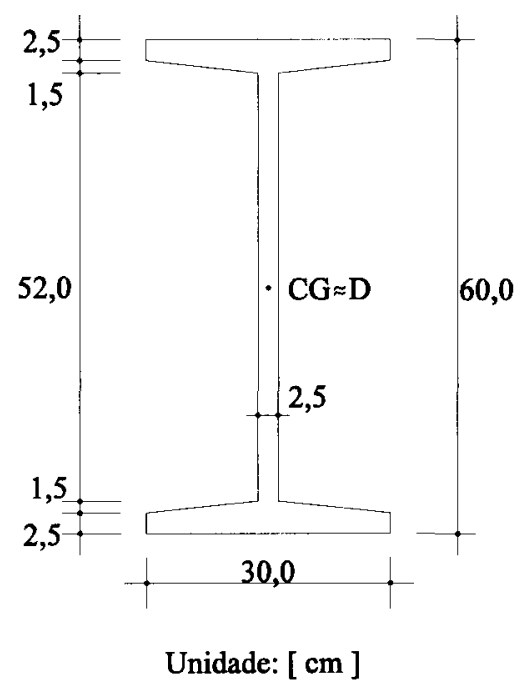

Figura 5.3 - Seção transversal da viga de argamassa armada .

As resistências características à tração e compressão da viga em estudo são:

$$
\begin{aligned}
f_{c k} & =3 \mathrm{kN} / \mathrm{cm}^{2} \\
f_{t k} & =0,06 \cdot f_{c k}+0,7 ; \text { para } f_{c k}[\mathrm{MPa}] \\
f_{t k} & =0,25 \mathrm{kN} / \mathrm{cm}^{2}
\end{aligned}
$$

e os módulos de elasticidade longitudinal e transversal:

$$
\begin{aligned}
& E_{\mathrm{sec}}=0,8 \cdot\left(0,9 \cdot 6600 \cdot \sqrt{f_{c k}+3,5}\right) \quad ; \quad f_{c k}[M P a] \\
& E=2750,40 \mathrm{kN} / \mathrm{cm}^{2} \\
& G=1100,16 \mathrm{kN} / \mathrm{cm}^{2}
\end{aligned}
$$

Para o peso específico da argamassa armada igual a $25 \mathrm{kN} / \mathrm{m}^{3}$, e a área da seção igual a $328,75 \mathrm{~cm}^{2}$, o peso próprio resulta igual a $8,2188 \times 10^{-3} \mathrm{kN} / \mathrm{cm}$. 
As características geométricas da viga de argamassa armada são:

$$
\begin{array}{ll}
I_{y}=14694,66 \mathrm{~cm}^{4} & I_{z}=189076,22 \mathrm{~cm}^{4} \\
J_{t}=965,21 \mathrm{~cm}^{4} & J_{w}=11,7752 \times 10^{6} \mathrm{~cm}^{6} \\
i_{D}^{2}=619,84 \mathrm{~cm}^{2} &
\end{array}
$$

onde $\mathrm{J}_{\mathrm{t}}$ é o momento de inércia à torção, $\mathrm{J}_{\mathrm{w}}$ é o momento setorial de inércia, $\mathrm{e} \mathrm{i}_{\mathrm{D}}$ é o raio de giração polar em relação ao centro de torção.

\subsubsection{FASE DE SERVIÇO}

A carga crítica em serviço da viga de argamassa armada pode ser calculada pela expressão de RACHID (1993), deduzida a partir da expressão da energia potencial total, utilizando o método de Ritz. A equação abaixo refere-se a uma viga esbelta de seção duplo T simétrico, apoiada nas extremidades sobre garfos indeformáveis, com carregamento linear uniformemente distribuído, sendo $\mathrm{d}^{*}$ a excentricidade do carregamento em relação ao centro de torção. Deve-se assumir a excentricidade positiva se o carregamento estiver aplicado acima do centro de torção. Neste exemplo, d* é igual a zero. Para a equação abaixo esquematizada, a carga crítica resulta em $0,0588 \mathrm{kN} / \mathrm{cm}$.

$$
p_{\text {crit }}=\frac{41,0415}{\ell^{4}} E I_{y} \cdot\left[ \pm \sqrt{4,74687 \frac{J_{w}}{I_{y}}+0,480957 \ell^{2} \frac{G J_{t}}{E I_{y}}+d^{\cdot 2}}-d \cdot\right]
$$

O cálculo da carga crítica para a viga em questão, através da expressão (2.36), resulta igual a $0,0550 \mathrm{kN} / \mathrm{cm}$, ou seja, desprezando as características setoriais da seção duplo T simétrico.

A relação $\mathrm{RCP}$ vale 7,15 , indicando a segurança da fase de serviço. 


\subsubsection{FASE TRANSITÓRIA DE SUSPENSÃO}

\subsubsection{Cabos inclinados e Cabos Retos}

Na aplicação do programa desenvolvido (Runge-Kutta) relativo à viga de seção duplo T simétrico, suspensa nas extremidades por cabos inclinados conforme ilustra a figura 5.4, tem-se na tabela 5.3, os resultados da carga crítica de instabilidade lateral para a variação do ângulo $\gamma$.

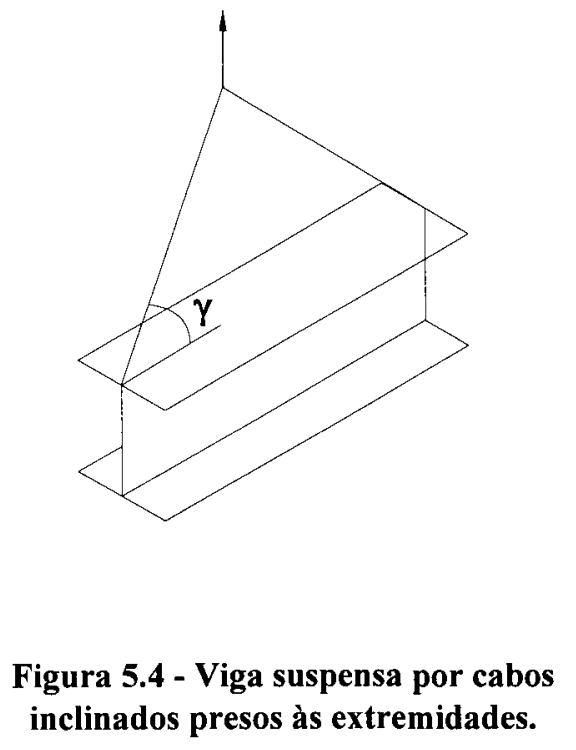

Tabela 5.3 - Carga crítica para a variação do ângulo $\gamma$ - Viga de argamassa armada

\begin{tabular}{||c|l||}
\hline$\gamma$ [graus] & $\mathrm{p}_{\text {crit }}[\mathrm{KN} / \mathrm{cm}]$ \\
\hline $50^{\circ}$ & $2,140 \times 10^{-2}$ \\
\hline $60^{\circ}$ & $2,200 \times 10^{-2}$ \\
\hline $70^{\circ}$ & $2,240 \times 10^{-2}$ \\
\hline $80^{\circ}$ & $2,280 \times 10^{-2}$ \\
\hline $90^{\circ}$ & $2,320 \times 10^{-2}$ \\
\hline $100^{\circ}$ & $2,370 \times 10^{-2}$ \\
\hline $110^{\circ}$ & $2,410 \times 10^{-2}$ \\
\hline $120^{\circ}$ & $2,470 \times 10^{-2}$ \\
\hline $130^{\circ}$ & $2,540 \times 10^{-2}$ \\
\hline
\end{tabular}

A suspensão por cabos retos sem balanços, de grande aplicação prática, é caso particular da disposição inclinada, e no programa para içamento por cabos retos, a carga crítica resulta também igual a $0,0232 \mathrm{kN} / \mathrm{cm}$. No içamento por cabos verticais, a relação $\mathrm{RCP}$, medida pela carga crítica e pelo peso próprio, é igual a 2,82. De forma geral, para a suspensão por cabos retos ou inclinados, a relação é inferior a 5,20 e a suspensão sem balanços não revela nenhuma segurança.

$\mathrm{Na}$ análise das tensões, a viga encontra-se fissurada, para os limites da 
resistência à tração do concreto indicados na norma brasileira NB-1. Isto significa que o estudo da instabilidade elástica não é adequado às tensões solicitantes que a viga apresenta.

O estudo de CATANIA \& COCCHI (1985) para suspensão com cabos retos, assumindo $\overline{\mathrm{d}}=1$, resulta em uma carga crítica de içamento equivalente a $0,0243 \mathrm{kN} / \mathrm{cm}$. Verifica-se na tabela 5.3, para $\gamma$ igual a $90^{\circ}$, valores próximos da carga crítica resultantes entre estes procedimentos de cálculo. Entretanto, sua indicação para as seções retangulares, parte do princípio de ter sido formulado baseado na equação diferencial de vigas sem características setoriais.

\subsubsection{Método Empírico}

Segundo a formulação de MAST (1993) para vigas protendidas de longos vãos, serão aplicados os mesmos procedimentos para avaliação da segurança, utililizados nesta referência bibliográfica, para uma adaptação em vigas sem protensão. As imperfeições construtivas serão consideradas neste estudo.

A suspensão da viga de argamassa armada será disposta com balanços para evitar ou apenas diminuir os riscos de fissuração.

Para a posição dos balanços que produz momentos fletores positivos e negativos iguais, tem-se o diagrama ilustrado na figura 5.5.

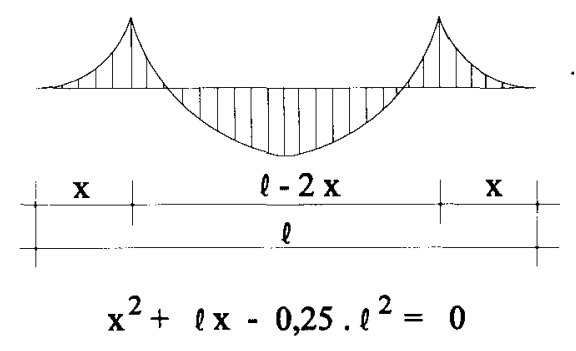

Figura 5.5 - Diagrama de momento fletor e equação para máximo momento fletor positivo e negativo iguais.

Sendo $\ell=15 \mathrm{~m}$, tem-se $\mathrm{x} \sim 3,10 \mathrm{~m}$. O momento fletor no meio do vão devido ao 
peso próprio de $8,21875 \cdot 10^{-3} \mathrm{kN} / \mathrm{cm}$ é:

$$
M_{p p}=400,66 \mathrm{kN} \mathrm{cm}
$$

Para o cálculo das tensões no meio do vão, tem-se:

$$
\begin{aligned}
& \sigma_{\text {mesa sup }}=-\frac{400,66}{189076,22} 30=-0,06357 \mathrm{kN} / \mathrm{cm}^{2} \\
& \sigma_{\text {mesa inf }}=0,06357 \mathrm{kN} / \mathrm{cm}^{2}
\end{aligned}
$$

$\mathrm{Na}$ verificação da resistência do concreto à tração, segundo as exigências da NB-1, a viga ainda encontra-se dentro dos limites desejados.

$$
0,06357 \frac{\mathrm{kN}}{\mathrm{cm}^{2}}<1,2 \cdot 0,25 \frac{\mathrm{kN}}{\mathrm{cm}^{2}}=0,30 \frac{\mathrm{kN}}{\mathrm{cm}^{2}}
$$

Uma maneira simples e aproximada de estimar analiticamente a excentricidade inicial decorrente das imperfeições construtivas, está proposta em MAST (1993). Esta excentricidade representa a distância entre o CG da viga fletida lateralmente, ilustrada por uma parábola do $2^{\circ}$ grau, à posição indeformada do elemento.

O PCI recomenda adotar para cada $3,05 \mathrm{~m}$ de comprimento da viga de concreto armado, 3,2mm de desvio lateral por imperfeições construtivas. MAST (1993) considera razoável a metade do valor indicado para o desvio lateral no PCI, e $6 \mathrm{~mm}$ para os desvios no posicionamento dos ganchos, onde devem ser acoplados os cabos de suspensão. Estes valores dependem basicamente do controle de qualidade da execução da fôrma de moldagem e não em especial, do material constituinte do elemento estrutural.

As indicações acima descritas para o concreto armado serão assumidas para a viga de argamassa armada.

A flecha lateral $\Delta_{z}$ é medida pela distância entre o deslocamento no meio do vão do eixo baricêntrico em relação às extremidades. $O$ cálculo da excentricidade inicial lateral pode ser descrito por: 


$$
\begin{aligned}
& e_{i}=\Delta_{z}\left[\left(\ell_{1} / \ell\right)^{2}-1 / 3\right]+6 \mathrm{~mm} \\
& \left(\frac{\ell_{1}}{\ell}\right)^{2}-\frac{1}{3}=\left(\frac{880}{1500}\right)^{2}-\frac{1}{3}=0,0108 \\
& e_{i}=7,9 \mathrm{~mm}(0,0108)+6 \mathrm{~mm}=6,1 \mathrm{~mm}
\end{aligned}
$$

onde por regra de três simples, obtém-se o valor da flecha lateral, segundo as indicações de MAST (1993).

A figura 5.6 ilustra as características geométricas da parábola.

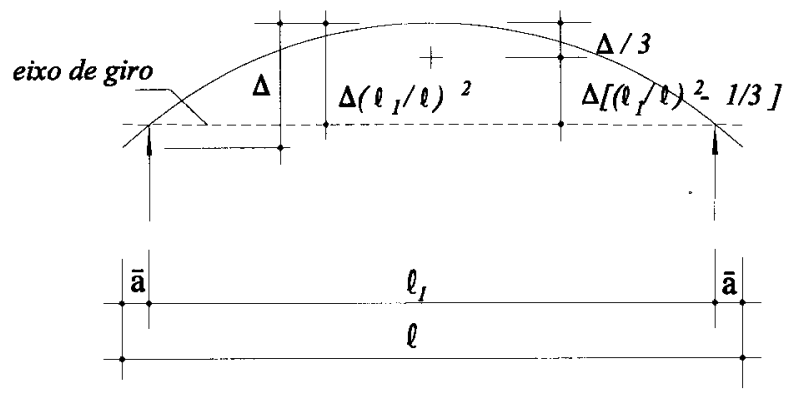

Figura 5.6 - Características geométricas da parábola para suspensão com balanços. [ MAST (1993) ]

Para o valor da curvatura $\mathrm{R}$, assumida como circular constante medida em relação às extremidades da viga, obtém-se a flecha no plano vertical y, e portanto, o valor de $\mathrm{y}_{\mathrm{r}}$ necessário ao cálculo do fator de segurança. A curvatura pode ser calculada por sua expressão aproximada:

$$
R=\frac{E I_{z}}{M}=\frac{2750,4 \cdot 189076,22}{400,66}=1297946,48 \mathrm{~cm}
$$

A flecha medida na vertical vale:

$$
\Delta_{y}=\frac{\ell^{2}}{8 R}=\frac{880^{2}}{8 \cdot 1297946,48}=0,07 \mathrm{~cm}
$$

sendo a distância $\mathrm{y}_{\mathrm{r}}$ do $\mathrm{CG}$ da viga ao eixo de giro definido na face superior da viga igual a:

$$
\begin{aligned}
& y_{r}=y_{t}+0,0108 \cdot \Delta_{y} \\
& y_{r}=30 \mathrm{~cm}+0,0108 \cdot 0,07 \mathrm{~cm} \sim 30,00 \mathrm{~cm}
\end{aligned}
$$


O cálculo do valor fictício $\mathrm{z}_{0}$, relativo ao deslocamento lateral do CG da viga para todo o carregamento aplicado lateralmente, na suspensão com balanços pode ser descrito por:

$$
\begin{aligned}
& z_{0}=\frac{p}{12 E I_{y} \ell}\left(\frac{1}{10} \ell_{1}^{5}-\overline{\mathbf{a}}^{2} \ell_{1}^{3}+3 \overline{\mathbf{a}}^{4} \ell_{1}+\frac{6}{5} \overline{\mathbf{a}}^{5}\right) \\
& z_{0}=\frac{8,21875 \cdot 10^{-3}}{12 \cdot 40416192,86 \cdot 1500}\left(\frac{1}{10} 880^{5}-310^{2} 880^{3}+3310^{4} 880+\frac{6}{5} 310^{5}\right) \\
& z_{0}=0,17 \mathrm{~cm}
\end{aligned}
$$

O giro inicial $\phi_{\mathrm{i}}$ por geometria, vale:

$$
\Phi_{i}=\frac{e_{i}}{y_{r}}=\frac{6,1 \mathrm{~mm}}{300,0 \mathrm{~mm}}=0,0203 \mathrm{rad}
$$

Para o cálculo do ângulo $\phi_{\text {máx }}$ relativo à fissuração, é necessário verificar a resistência à tração do concreto e calcular o máximo momento lateral resistente. Por geometria, determina-se o máximo ângulo de inclinação $\phi$.

A máxima tensão de tração atuante na argamassa armada é $0,06357 \mathrm{kN} / \mathrm{cm}^{2} \mathrm{e}$ a máxima tensão resistente de tração é $0,3 \mathrm{kN} / \mathrm{cm}^{2}$.

$$
M_{\text {lat }}=\frac{(0,3-0,06357) \cdot 14694,66}{\frac{30}{2}}=231,62 \mathrm{kN} . \mathrm{cm}
$$

O máximo ângulo ф relativo à fissuração é:

$$
\phi_{\text {max }}=\frac{M_{l a t}}{M_{p p}}=\frac{231,62}{400,66}=0,5781 \mathrm{rad}
$$

O cálculo do fator de segurança FS contra fissuração a partir da expressão (4.22) definida no capítulo 4, resulta em: 


$$
\begin{gathered}
F S=\frac{1}{\frac{z_{0}}{y_{r}}+\frac{\phi_{i}}{\phi_{\text {max }}}}=\frac{1}{\frac{0,17}{30,00}+\frac{0,0203}{0,5781}} \\
F S=24,52
\end{gathered}
$$

Na verificação da segurança contra ruptura, deve-se calcular o ângulo $\phi_{\text {máx }}^{\prime}$ de ruptura e o valor de $z_{0}^{\prime}$. Assim:

$$
\begin{gathered}
\phi_{\text {max }}^{\prime}=\sqrt{\frac{e_{i}}{2,5 \cdot z_{0}}}=\sqrt{\frac{6,1 \mathrm{~mm}}{2,5 \cdot 1,7 \mathrm{~mm}}}=1,1981 \mathrm{rad} \\
z_{0}^{\prime}=z_{0}\left(1+2,5 \phi_{\text {max }}^{\prime}\right)=0,68 \mathrm{~cm} \\
F S^{\prime}=\frac{y_{r} \cdot \phi_{\text {max }}^{\prime}}{z_{0}^{\prime} \cdot \phi_{\text {max }}^{\prime}+e_{i}}=\frac{30 \cdot 1,1981}{0,68 \cdot 1,1981+0,61} \\
F S^{\prime}=25,23
\end{gathered}
$$

Utilizou-se a inércia efetiva para a viga fissurada, conforme expressão recomendada por MAST (1993) para as vigas de concreto armado. Ensaios para avaliar a perda de rigidez lateral das vigas de argamassa armada após a fissuração devem ser realizados.

Como observa-se pelo alto resultado do fator de segurança contra fissuração, a presença dos balanços garante a segurança desta fase, também confirmada pela verificação das tensões.

O estudo de CATANIA \& COCCHI (1985) apresentado no item 3.2.2, para a suspensão pelos quartos de vão, resulta em uma carga crítica igual a $0,8428 \mathrm{kN} / \mathrm{cm}$. Esta carga crítica fornece uma relação RCP igual a 102,55 muito superior ao limite mínimo estabelecido igual a 5,20. O estudo empírico, para ește mesmo comprimento de balanços, fornece um fator de segurança equivalente a 17,61.

Entretanto, não é possível estabelecer maiores comparações entre os processos citados, visto que apresentam diferentes formas de introduzir a segurança. Por outro lado, em ambas as análises, verifica-se a segurança do içamento do elemento prémoldado com balanços, onde as tensões atuantes são inferiores às tensões limites. 


\subsection{VIGA PROTENDIDA PCI BT-72}

A viga protendida padrão do PCI, designada por PCI BT-72, é uma viga usual duplo T não simétrico com 72 polegadas $(1,83 \mathrm{~m})$ de altura, $41,45 \mathrm{~m}$ de comprimento e cuja seção transversal encontra-se ilustrada na figura 5.7. As análises a serem desenvolvidas baseiam-se na viga exemplificada em MAST (1993) e em IMPER \& LASZLO (1987).

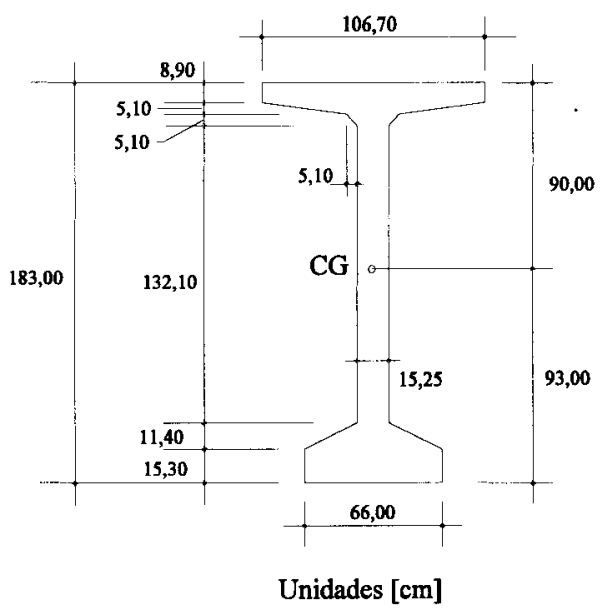

Figura 5.7 - Seção transversal viga PCI BT-72.

A protensão e a respectiva excentricidade em relação à linha do CG estão representadas na figura 5.8 .

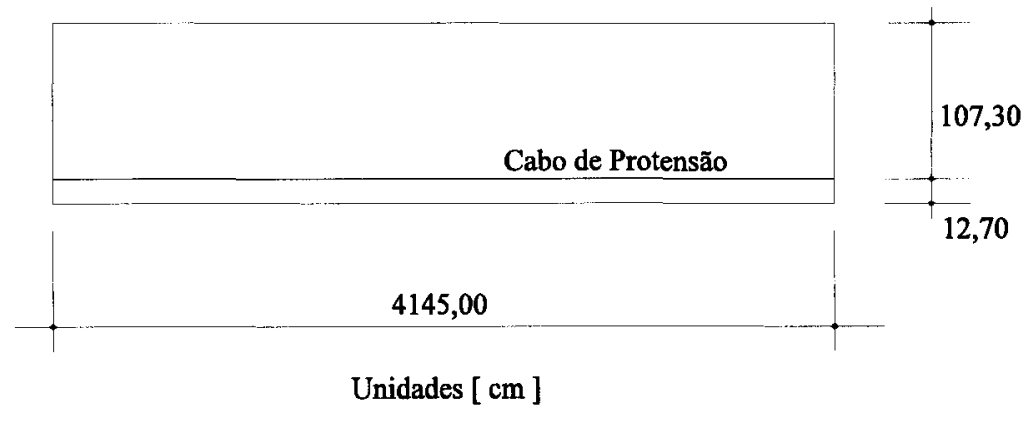

Figura 5.8 - Disposição do cabo de protensão na viga pré-moldada de concreto.

A posição do centro de gravidade é $90 \mathrm{~cm}$ a partir da superfície superior e 93 
cm da base da seção. A força de protensão aplicada após as perdas imediatas é igual a $4500 \mathrm{kN}$, cuja excentricidade $\mathrm{e}_{0}$ vale $80,3 \mathrm{~cm}$.

As características geométricas da seção transversal são:

$$
\begin{aligned}
& A=0,4950 \mathrm{~m}^{2} \\
& I_{z}=0,227218 \mathrm{~m}^{4} \\
& I_{y}=0,015664 \mathrm{~m}^{4}
\end{aligned}
$$

e as propriedades do concreto:

$$
\begin{aligned}
& f^{\prime}{ }_{c i}=31 \mathrm{MPa}=31000 \mathrm{kN} / \mathrm{m}^{2} \\
& \gamma_{c}=2483 \mathrm{Kg} / \mathrm{m}^{3} \quad\left(w_{c}=155 \mathrm{lb} / \mathrm{ft}^{3}\right) \\
& E=29454,5 \mathrm{MPa}
\end{aligned}
$$

sendo o módulo de elasticidade E calculado utilizando a mesma equação indicada por MAST (1993), equivalente à expressão apresentada no ACI:

$$
\begin{aligned}
& E=33 \cdot w_{c}^{1,5} \cdot f_{c i}^{\prime 0,5}
\end{aligned}
$$

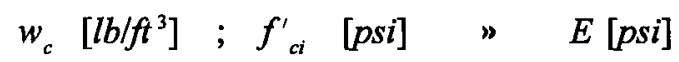

onde $\mathrm{w}_{\mathrm{c}}$ equivale ao peso específico aparente do concreto armado e $\mathrm{f}_{\mathrm{ci}}^{\prime}$ é a resistência inicial de um corpo de prova cilíndrico no instante da aplicação da protensão.

As expressões utilizadas para cálculo do módulo de elasticidade neste item são as mesmas usadas em MAST (1993), segundo o ACI, para facilitar comparações com o trabalho referido.

\subsubsection{FASE DE SERVIÇO}

Segundo a expressão (2.78) para vínculos de garfo indeformável à torção, uma análise da carga crítica pode ser desenvolvida como segue:

$$
p^{*}{ }_{\text {crt }}=-\frac{G J_{t}}{n\left(e_{0}^{2}-i_{z}^{2}\right)-\frac{4 d \cdot l^{2}}{\pi^{3}}}
$$




$$
p_{\text {crit }}=-\frac{53524,482}{n\left(0,803^{2}-0,459\right)-\frac{4 \cdot 0,42 \cdot 41,45^{2}}{\pi^{3}}}
$$

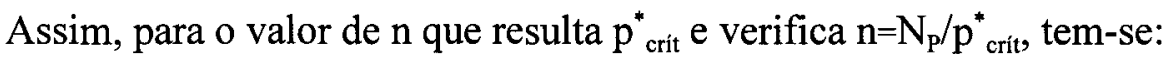

$$
\begin{aligned}
& n=7,706 \rightarrow p_{c r t}^{\cdot}=583,950 \mathrm{kN} / \mathrm{m} ; N_{P}=4500 \mathrm{kN} \\
& p_{0}=\frac{4}{\pi} p_{c r t t}=743,508 \mathrm{kN} / \mathrm{m}
\end{aligned}
$$

onde $\mathrm{p}_{0}$ representa carregamento linear uniformemente distribuído e é proveniente da aplicação das séries de Fourier na definição do tipo de carregamento.

A relação RCP fornece uma comparação entre a carga crítica e o peso próprio:

$$
R C P=\frac{p_{0}}{p p}=\frac{743,508}{12,291}=60,50
$$

Esta fase, segundo o trabalho de CATANIA \& COCCHI (1985), apresenta alto índice de segurança em relação ao carregamento exclusivo do peso próprio.

\subsubsection{FASE TRANSITÓRIA DE SUSPENSÃO}

\subsubsection{Cabos Inclinados}

A formulação de CATANIA \& COCCHI (1985) para a suspensão com cabos inclinados presos às extremidades foi desenvolvida pelo método de Ritz aplicado à expressão da energia potencial total.

Para a suspensão com cabos inclinados, conforme estudo descrito no capítulo 3, a expressão (3.87) para cálculo da carga crítica de suspensão em função do tipo de carregamento é:

$$
p_{\text {crit }}^{*}=\frac{\pi^{7} z_{1}^{2}}{64 E I_{y} d^{3} \operatorname{tg}^{2} \gamma \ell^{2}}\left[G J_{t}+N_{p}\left(e_{0}^{2}-i_{x}^{2}\right)\right]^{2}
$$

onde: $\quad \mathrm{z}_{1}$ - distância do CG à face superior da viga;

$\mathrm{d}^{*}$ - distância do ponto de aplicação do carregamento ao centro de torção; 
$\gamma$ - ângulo de inclinação do cabo de suspensão com a face superior da viga;

$\ell$ - vão da viga;

$\mathrm{N}_{P}$ - força de protensão;

$\mathrm{e}_{0}$ - excentricidade do cabo de protensão em relação ao $\mathrm{CG}$;

$\mathrm{i}_{\mathrm{z}}$ - raio de giração: $\left(\mathrm{I}_{\mathrm{z}} / \mathrm{A}\right)^{0,5}$.

Assim, para os dados da viga protendida do PCI BT-72:

$$
\begin{gathered}
p_{\text {crit }}=\frac{\pi^{7} 0,90^{2}}{64.461375,288 \cdot 0,42^{3} \cdot \operatorname{tg}^{2} \gamma \cdot 41,45^{2}}\left[53524,482+4500\left(0,803^{2}-0,459\right)\right]^{2} \\
p^{\cdot}{ }_{\text {crt }}=\frac{1923,409}{\operatorname{tg}^{2} \gamma}[\mathrm{kN} / \mathrm{m}]
\end{gathered}
$$

Na tabela 5.4, para diversos valores do ângulo de inclinação do cabo de suspensão, resultam os valores da carga crítica em função do tipo de carregamento, representado pelas séries de Fourier.

Tabela 5.4 - Valores da carga crítica em função do ângulo $\gamma$ - Viga protendida PCI BT-72.

\begin{tabular}{|c|c|}
\hline$\gamma$ [graus] & $\mathrm{p}^{*}{ }_{\text {crit }}[\mathrm{kN} / \mathrm{m}]$ \\
\hline $45^{\circ}$ & 1923,41 \\
\hline $50^{\circ}$ & 1354,25 \\
\hline $60^{\circ}$ & 641,14 \\
\hline $70^{\circ}$ & 254,80 \\
\hline $80^{\circ}$ & 59,80 \\
\hline $85^{\circ}$ & 14,72 \\
\hline $89,9^{\circ}$ & 0,0059 \\
\hline
\end{tabular}

A formulação acima descrita apresenta algumas limitações. Para variações do ângulo de inclinação $\gamma$ dos cabos de içamento nos limites de $90^{\circ}$, a carga crítica resulta 
próximo e tendendo a zero. Além disso, fazendo a componente normal $\mathrm{F}_{0}$ da força de içamento para cabos inclinados igual a zero, ou seja, suspensão com cabos retos, a carga crítica nesta fase transitória equivale à carga crítica de serviço. Para valores proporcionais de $\gamma<90^{\circ} \mathrm{e} \gamma>90^{\circ}$, a carga crítica é a mesma.

A aproximação desta formulação, desenvolvida por CATANIA \& COCCHI (1985), resulta da não consideração dos giros por torção e dos deslocamentos laterais da seção do apoio. É como se a fase de suspensão apenas acrescentasse a componente normal resultante do içamento por cabos inclinados.

Observa-se que, na disposição dos cabos nas extremidades formando ângulos menores que $90^{\circ}$, a carga crítica aumenta, o que não ocorre nas vigas sem protensão.

\subsubsection{Cabos Retos}

\subsection{Sem balanços}

O estudo da viga PCI BT-72 na fase de suspensão será numericamente realizado para o içamento por cabos retos sem balanços.

Para o peso específico do concreto armado, tem-se o peso-próprio da viga:

$$
p p=12,291 \mathrm{kN} / \mathrm{m}
$$

O momento fletor devido ao peso-próprio vale:

$$
M_{p p}=12,291.41,45^{2}\left[\frac{1}{4}-\frac{1}{8}\right]=2639,650 \mathrm{kN} / \mathrm{m}
$$

e as tensões normais atuantes nas mesas:

$$
\begin{aligned}
& \sigma_{\text {mess sup }}=-\frac{4500}{0,495}+\frac{4500 \cdot 0,803}{0,227218} 0,90-\frac{2639,650.0,90}{0,227218}=-5233,534 \frac{\mathrm{kN}}{\mathrm{m}^{2}} \\
& \sigma_{\text {mesa inf }}=-\frac{4500}{0,495}-\frac{4500.0,803}{0,227218} 0,93+\frac{2639,650 \cdot 0,93}{0,227218}=-13076,863 \frac{\mathrm{kN}}{\mathrm{m}^{2}}
\end{aligned}
$$

A ilustração das tensões normais devido à protensão e ao peso-próprio encontra-se esquematizada na figura 5.9. 


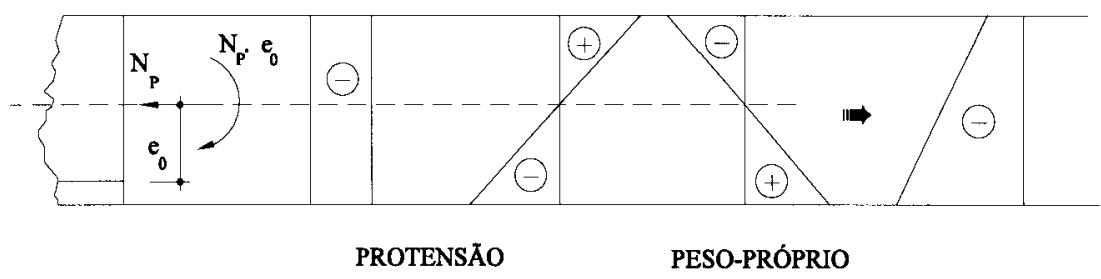

Figura 5.9 - Diagrama das tensões normais atuantes na seção do meio do vão para suspensão nas extremidades.

A verificação da resistência do concreto, conforme realizada em MAST (1993), foi feita majorando a máxima tensão atuante:

$$
{f^{\prime}{ }_{{ }_{\text {requerida }}}^{\prime}}=\frac{13076,863}{0,6}=21794,772 \frac{\mathrm{kN}}{\mathrm{m}^{2}}<31000 \frac{\mathrm{kN}}{\mathrm{m}^{2}}
$$

Não sendo necessário modificar a resistência inicial do concreto, o valor do módulo de elasticidade E continua o mesmo.

O cálculo da excentricidade inicial lateral baseia-se nas indicações do PCI para os desvios laterais construtivos e nas recomendações de MAST (1993), as quais já foram citadas no exemplo da viga de argamassa armada.

A excentricidade inicial, medida em relação ao arco descrito por uma parábola, pela distância da posição indeformada ao CG da linha, para suspensão nos extremos é:

$$
\begin{aligned}
& e_{i}=\frac{2}{3} \Delta_{z}+6 \mathrm{~mm} \\
& e_{i}=\frac{2}{3} \cdot 21,7+6=20,5 \mathrm{~mm} \\
& \text { onde : } \quad \begin{array}{c}
3,05 m-1,6 \mathrm{~mm} \\
41,45 \mathrm{~m}-
\end{array}
\end{aligned}
$$

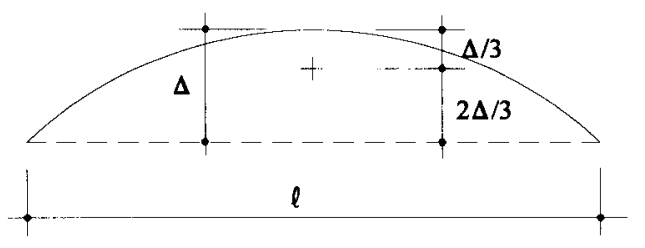

Figura 5.10 - Propriedades da parábola para suspensão nas extremidades.

A estimativa da curvatura no plano vertical assumida circular e constante, medida em relação às extremidades da viga, pela expressão aproximada, resulta em: 


$$
\frac{1}{R}=\frac{M}{E I_{z}} \quad \text { para }: \quad M=N_{p} \cdot e_{0}-M_{p p}
$$

onde o momento fletor $M$ no meio do vão é:

$$
M=4500.0,803-2639,650=973,850 \mathrm{kNm}
$$

Assim, a curvatura vale:

$$
R=\frac{29454500 \cdot 0,227218}{973,850}=6872,303 \mathrm{~m}
$$

O cálculo da flecha vertical $\Delta_{y}$, para curvatura constante:

$$
\Delta_{y}=\frac{\ell^{2}}{8 R}=\frac{41,45^{2}}{8.6872,303}=0,0313 m
$$

Como a flecha no meio do vão resulta da influência predominante da protensão em relação ao peso-próprio, para o cálculo de $\mathrm{y}_{\mathrm{r}}$, tem-se:

$$
y_{r}=y_{t}-\frac{2}{3} \Delta_{y}=0,90-\frac{2}{3} \cdot 0,0313=0,8791 \mathrm{~m}
$$

A curvatura tem apenas um pequeno efeito sobre $\mathrm{y}_{\mathrm{r}}$. Sugere-se então, ao invés dos cálculos acima, subtrair de $\mathrm{y}_{\mathrm{r}}$ uma quantidade em torno de 25 a $50 \mathrm{~mm}$.

O cálculo de $z_{0}$, para suspensão nas extremidades, é obtido através da seguinte expressão:

$$
z_{0}=\frac{p \ell^{4}}{120 E I_{y}}=\frac{12,291 \cdot 41,45^{4}}{120 \cdot 29454500 \cdot 0,015664}=0,6553 \mathrm{~m}
$$

Para $e_{i}$ e $y_{r}$ calculados, o valor de $\phi_{\mathrm{i}}$ resulta da relação geométrica:

$$
\phi_{i}=\frac{e_{i}}{y_{r}}=\frac{20,5 \mathrm{~mm}}{879,1 \mathrm{~mm}}=0,0233 \mathrm{rad}
$$

A partir da verificação da resistência à tração do concreto indicada no $\mathrm{ACI}$, e que será neste exemplo da mesma forma realizada, determina-se o máximo momento lateral resistente, e também por relações geométricas, calcula-se o ângulo $\phi_{\operatorname{máx}}$ de fissuração.

O limite indicado no ACI para a resistência à tração do concreto é: 


$$
\begin{aligned}
& f_{r}=7,5 \cdot \sqrt{f_{c i}^{\prime}} \quad \text { para } f_{c i}^{\prime} \text { em }[p s i] \\
& f_{r}=7,5 \cdot \sqrt{4500 p s i} ; \text { pois } f_{c i}^{\prime}=31000 \mathrm{kN} / \mathrm{m}^{2}=4500 \mathrm{psi} \\
& f_{r}=503,115 \mathrm{psi}=3468,980 \mathrm{kN} / \mathrm{m}^{2}
\end{aligned}
$$

Como as tensões atuantes são de compressão, a verificação do limite de tração será feito para a menor tensão de compressão, que equivale a $5233,534 \mathrm{kN} / \mathrm{m}^{2}$ na mesa superior.

O máximo momento lateral resistente deve ser então calculado por:

$$
M_{\text {lat }}=\frac{\sigma \cdot I_{y}}{y}=\frac{(3468,980+5233,534) \cdot 0,015664}{\frac{1,067}{2}}=255,513 \mathrm{kNm}
$$

e o máximo ângulo $\phi$ de fissuração é:

$$
\phi_{\max }=\frac{M_{\text {lat }}}{M_{p p}}=\frac{255,513 \mathrm{kNm}}{2639,650 \mathrm{kNm}}=0,0968 \mathrm{rad}
$$

O cálculo de $\phi_{\text {máx }}$ foi desenvolvido para o momento fletor devido ao peso próprio, o que é a favor da segurança, porque a protensão diminue o momento resultante.

O fator de segurança FS contra a fissuração é, enfim:

$$
F S=\frac{1}{\frac{z_{0}}{y_{r}}+\frac{\phi_{i}}{\phi_{\max }}}=\frac{1}{\frac{0,65531 \mathrm{~m}}{0,87917 \mathrm{~m}}+\frac{0,0233 \mathrm{rad}}{0,0968 \mathrm{rad}}}=1,01
$$

logo, para as indicações recomendadas em MAST (1993), para valores de fissuração, a suspensão apresenta segurança mínima. Mas, em relação às indicações de IMPER \& LASZLO (1987), que recomendam um coeficiente maior ou igual a 1,5 para manuseio no canteiro da fábrica e 1,75 no local na edificação, os índices recomendados não se verificam.

Na determinação do fator de segurança contra a ruptura FS', deve-se calcular o máximo ângulo $\phi_{\text {máx }}^{\prime}$ de ruptura. Assim: 


$$
\phi_{\text {max }}^{\prime}=\sqrt{\frac{e_{i}}{2,5 \cdot z_{0}}}=\sqrt{\frac{20,5 \mathrm{~mm}}{2,5.655,3 \mathrm{~mm}}}=0,1119 \mathrm{rad}
$$

e calculando $z^{\prime}{ }_{0}$ para a ruptura, tem-se:

$$
z_{0}^{\prime}=z_{0}\left(1+2,5 \cdot \phi_{\text {max }}^{\prime}\right)=0,6553(1+2,5 \cdot 0,1119)=0,8386 m
$$

$\mathrm{O}$ fator de segurança $\mathrm{FS}^{\prime}$ contra ruptura pode ser obtido pela seguinte expressão:

$$
F S^{\prime}=\frac{y_{r} \cdot \Phi_{\max }^{\prime}}{z_{0}^{\prime} \cdot \phi_{\max }^{\prime}+e_{i}}=\frac{0,8791 \cdot 0,1119}{0,8386 \cdot 0,1119+0,0205}=0,86
$$

que está fora das recomendações e é menor que 1,5. Deve-se então tomar o fator de segurança contra ruptura igual ao fator de segurança contra fissuração.

Assim, a suspensão nas extremidades resulta em mínimas condições de segurança segundo MAST (1993), mas está fora dos coeficientes recomendados por IMPER \& LASZLO (1987).

\subsection{Com balanços}

A definição da posição dos balanços pode ser feita conforme a figura 4.15. Adotando o fator de segurança $F S=1,5$ para manuseio, seguindo as orientações de IMPER \& LASZLO (1987), a flecha no meio do vão para apoios nas extremidades vale:

$$
\Delta_{y}=\frac{5 \cdot p \ell^{4}}{384 \cdot E I_{y}}=\frac{5 \cdot 12,291 \cdot 41,45^{4}}{384 \cdot 29454500 \cdot 0,015664}=1,024 \mathrm{~m}
$$

A razão entre as flechas com e sem balanços é dada por:

$$
\begin{gathered}
\beta_{y}=\frac{y_{t}}{F S}=\frac{0,90 \mathrm{~m}}{1,5}=0,60 \mathrm{~m} \\
\frac{\beta_{y}}{\Delta_{y}}=\frac{0,60 \mathrm{~m}}{1,03 \mathrm{~m}}=0,583
\end{gathered}
$$

e para determinar o comprimento do balanço a partir do fator de segurança imposto, 
tem-se que a relação $\beta_{y} / \Delta_{y}=0,583$ na figura 4.15 , define a razão balanço/vão representada por $\bar{a} / \ell$, aproximadamente igual a 0,06 . Assim:

$$
\begin{gathered}
\overline{\mathbf{a}}=0,06 \cdot \ell=0,06 \cdot 41,45=2,487 \mathrm{~m} \\
\ell_{1}=41,45-2 \cdot 2,487=36,476 \mathrm{~m}
\end{gathered}
$$

Arredondando os comprimentos dos vãos e balanços, tem-se:

$$
\overline{\mathrm{a}}=2,50 \mathrm{~m} \quad \mathrm{e} \quad \ell_{1}=36,45 \mathrm{~m}
$$

onde $\ell_{1}=\ell-2 \bar{a}$.

Verificando a flecha para suspensão com balanços, resulta:

$$
\begin{aligned}
& \beta_{y}=\frac{p \cdot \ell_{1}{ }^{2}}{384 \cdot E I_{y}}\left(5 \ell_{1}^{2}-24 \overline{\mathbf{a}}^{2}\right) \\
& \beta_{y}=\frac{12,291 \cdot 36,45^{2}}{384 \cdot 29454500 \cdot 0,015664}\left(5 \cdot 36,45^{2}-24 \cdot 2,50^{2}\right) \\
& \beta_{y}=0,598 m
\end{aligned}
$$

onde o fator de segurança vale:

$$
F S=\frac{y_{t}}{\beta_{y}}=\frac{0,900 m}{0,598 m}=1,50 \geq 1,50
$$

O cálculo das tensões na seção do meio do vão para os efeitos do peso próprio e da protensão deve ser realizado para verificar as tensões atuantes e as resistentes.

$$
M_{p p}=\frac{p}{2}\left(\ell_{1} \cdot x-x^{2}-\overline{\mathrm{a}}^{2}\right)=1964,413 k N m
$$

Para a força normal de protensão igual a $4500 \mathrm{kN}$ com excentricidade $\mathrm{e}_{0}$ igual a $0,803 \mathrm{~m}$, tem-se o cálculo das tensões normais atuantes para o diagrama ilustrado na figura 5.11.

$$
\begin{aligned}
& \sigma_{\text {mesa sup }}=-\frac{4500}{0,495}+\frac{4500 \cdot 0,803}{0,227218} 0,90-\frac{1964,413 \cdot 0,90}{0,227218}=-2558,952 \frac{\mathrm{kN}}{\mathrm{m}^{2}} \\
& \sigma_{\text {mesa inf }}=-\frac{4500}{0,495}-\frac{4500 \cdot 0,803}{0,227218} 0,93+\frac{1964,413 \cdot 0,93}{0,227218}=-15840,598 \frac{\mathrm{kN}}{\mathrm{m}^{2}}
\end{aligned}
$$

A verificação da resistência do concreto para a majoração da tensão atuante, resulta em: 


$$
f_{c i}=\frac{15840,598 \mathrm{kN} / \mathrm{m}^{2}}{0,6}=26400,997 \mathrm{kN} / \mathrm{m}^{2}<31000 \mathrm{kN} / \mathrm{m}^{2}
$$

não sendo necessário alterar o valor do módulo de elasticidade $\mathrm{E}$.

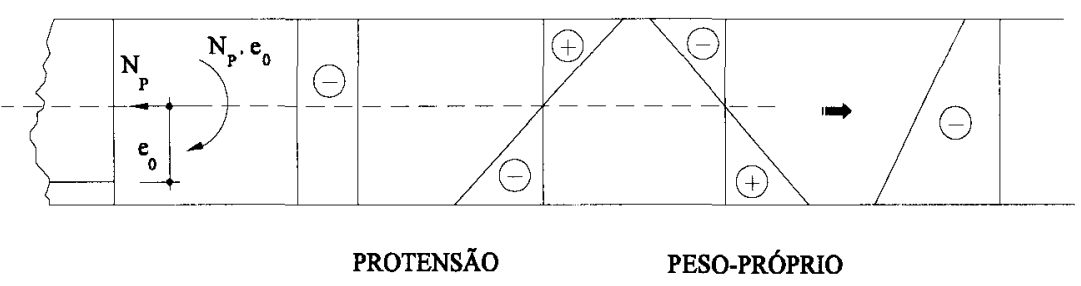

Figura 5.11 - Diagrama das tensões normais atuantes na seção do meio do vão para suspensão com balanços.

A excentricidade inicial medida lateralmente, para as propriedades da parábola em relação ao CG da linha, na suspensão com balanços é:

$$
\begin{aligned}
& \left(\ell_{1} / \ell\right)-1 / 3=\left(\frac{36,45}{41,45}\right)^{2}-\frac{1}{3}=0,440 \\
& e_{i}=21,7 \mathrm{~mm}(0,440)+6 \mathrm{~mm}=15,5 \mathrm{~mm}
\end{aligned}
$$

A curvatura circular e constante em relação às extremidades da viga, para o momento fletor M no meio do vão considerando a protensão e o peso próprio, pode ser obtida por:

$$
\begin{gathered}
M=1610,678 \mathrm{kNm} \\
R=\frac{29454500 \cdot 0,227218}{1610,678}=4155,140 \mathrm{~m}
\end{gathered}
$$

e a flecha vertical:

$$
\Delta_{y}=\frac{\ell^{2}}{8 R}=\frac{41,45^{2}}{8 \cdot 4155,140}=0,052 m
$$

Para a contra-flecha devido à protensão, o cálculo de $\mathrm{y}_{\mathrm{r}}$ resulta em:

$$
y_{r}=y_{t}-0,440 . \Delta_{y}=0,90-0,440 \cdot 0,052=0,877 m
$$

e o cálculo de $\mathrm{z}_{0}$, para suspensão com balanços: 


$$
\begin{gathered}
z_{0}=\frac{p}{12 E I_{y} \ell}\left(\frac{1}{10} \ell_{1}^{5}-\overline{\mathbf{a}}^{2} \ell_{1}^{3}+3 \overline{\mathrm{a}}^{4} \ell_{1}+\frac{6}{5} \overline{\mathbf{a}}^{5}\right) \\
z_{0}=\frac{12,291}{12 \cdot 461375,288 \cdot 41,45}\left(\frac{1}{10} 36,45^{5}-2,5^{2} \cdot 36,45^{3}+3 \cdot 2,5^{4} \cdot 36,45+\frac{6}{5} 2,5^{5}\right) \\
z_{0}=0,329 \mathrm{~m}
\end{gathered}
$$

Da relação geométrica entre $e_{i}$ e $y_{r}$, tem-se $\phi_{i}$ :

$$
\phi_{i}=\frac{e_{i}}{y_{r}}=\frac{15,5 \mathrm{~mm}}{877,0 \mathrm{~mm}}=0,0177 \mathrm{rad}
$$

Para o limite indicado no $\mathrm{ACI}$, a resistência à tração do concreto é:

$$
\begin{aligned}
& f_{r}=7,5 \cdot \sqrt{f_{c i}^{\prime}} \quad \text { para } f_{c i}^{\prime} \text { em }[\mathrm{psi}] \\
& f_{r}=7,5 \cdot \sqrt{4500 \mathrm{psi}} ; \text { pois } f_{c i}^{\prime}=31000 \mathrm{kN} / \mathrm{m}^{2}=4500 \mathrm{psi} \\
& f_{r}=503,115 \mathrm{psi}=3468,980 \mathrm{kN} / \mathrm{m}^{2}
\end{aligned}
$$

e para a menor tensão de compressão $2558,952 \mathrm{kN} / \mathrm{m}^{2}$ na mesa superior, o máximo momento lateral resistente pode ser calculado por:

$$
M_{\text {lat }}=\frac{\sigma \cdot I_{y}}{y}=\frac{(3468,980+2558,952) \cdot 0,015664}{\frac{1,067}{2}}=176,985 \mathrm{kNm}
$$

sendo o máximo ângulo $\phi$ de fissuração:

$$
\phi_{\text {max }}=\frac{M_{\text {lat }}}{M_{p p}}=\frac{176,985 \mathrm{kNm}}{1964,413 \mathrm{kNm}}=0,0901 \mathrm{rad}
$$

O fator de segurança FS contra a fissuração vale, enfim:

$$
F S=\frac{1}{\frac{z_{0}}{y_{r}}+\frac{\phi_{i}}{\phi_{\text {mix }}}}=\frac{1}{\frac{0,329 \mathrm{~m}}{0,877 \mathrm{~m}}+\frac{0,0177 \mathrm{rad}}{0,0901 \mathrm{rad}}}=1,75>1,5
$$

O coeficiente de segurança FS contra fissuração supera os valores mínimos recomendados, os quais foram citados anteriormente, na suspensão sem balanços.

No cálculo do fator de segurança contra ruptura FS', o máximo ângulo $\phi_{\text {máx }}^{\prime}$ de ruptura é: 


$$
\phi_{\text {max }}^{\prime}=\sqrt{\frac{e_{i}}{2,5 \cdot z_{0}}}=\sqrt{\frac{0,0155 \mathrm{~m}}{2,5 \cdot 0,3290 \mathrm{~m}}}=0,1373 \mathrm{rad}
$$

e $\mathrm{z}_{0}^{\prime}$ para a ruptura:

$$
z_{0}^{\prime}=z_{0}\left(1+2,5 \cdot \phi_{\text {mix }}^{\prime}\right)=0,329(1+2,5 \cdot 0,1373)=0,4419 m
$$

Assim:

$$
F S^{\prime}=\frac{y_{r} \cdot \phi_{\max }^{\prime}}{z_{0}^{\prime} \cdot \phi_{\text {max }}^{\prime}+e_{i}}=\frac{0,877 \cdot 0,1373}{0,4419 \cdot 0,1373+0,0155}=1,58<F S
$$

que por ser menor que FS deve-se ter $\mathrm{FS}^{\prime}=\mathrm{FS}$. Apesar do fator de segurança contra ruptura ser inferior ao fator de segurança contra fissuração, é superior ao mínimo estabelecido por MAST (1993), igual a 1,5.

A disposição dos cabos com balanços resulta em fatores de segurança superiores aos estabelecidos como mínimos e bem mais folgados em relação aos resultantes do içamento pelas extremidades.

Da verificação das tensões nota-se o melhor comportamento da viga pela presença dos balanços, na suspensão da viga protendida. 


\section{CONSIDERAÇÕES FINAIS E CONCLUSÕES}

Devido às situações de colapso e fissuração das vigas pré-moldadas esbeltas e de longos vãos resultante da instabilidade lateral destes elementos, procurou-se reunir neste trabalho, o estudo do equilíbrio no espaço em regime elástico-linear das vigas, durante a fase de serviço e a fase transitória.

Alguns comentários relativos às análises dos exemplos numéricos definidas no capítulo 5 merecem novo destaque.

Quanto ao comportamento das vigas durante o regime de serviço confirmou-se que estas fases geralmente não são críticas por perda de estabilidade lateral. $\mathrm{Na}$ fase transitória de suspensão sem balanços verificam-se situações de flambagem lateral, como é o caso da viga esbelta de argamassa armada mostrada no item 5.3. A presença dos balanços incrementa consideravelmente a segurança desta fase, como verificado pelo procedimento empírico de MAST (1993), no qual comprova-se a inexistência de fissuras.

A presença dos balanços aumenta a segurança da fase transitória, tanto para as vigas protendidas como para as vigas sem protensão. Porém, o comprimento dos balanços das vigas protendidas são geralmente pequenos, por apresentarem a situação em vazio. Confirma-se que, pelos exemplos analisados, a melhor disposição dos cabos 
para as vigas sem protensão situa-se, geralmente, nas proximidades dos quartos de vão, o que intuitivamente já era esperado.

Quanto aos processos de cálculo, a formulação de CATANIA \& COCCHI (1985) contém uma série de aproximações e limitações, que serão detalhadas a seguir, mas tem o seu mérito por avaliar, ainda que aproximadamente, a instabilidade para diversas disposições dos cabos de suspensão. Os programas desenvolvidos neste trabalho mostram-se mais adequados ao estudo da carga crítica de instabilidade de peças delgadas, sendo específicos para algumas seções. O procedimento empírico tratado por MAST (1993) representa uma maneira simples de verificar a segurança através do cálculo dos fatores de segurança.

É necessário esclarecer algumas aproximações do estudo de CATANIA \& COCCHI (1985) de forma a melhorar sua aplicação. Em vigas delgadas, onde faz-se necessário considerar as características setoriais da seção transversal, o estudo da instabilidade durante a fase de suspensão deve ser aplicado às seções retangulares, seções $\mathrm{T}$ e seções cruz, nas quais o diagrama de área setorial é nulo. Isto porque, as expressões finais da carga crítica, para as diversas formas de içamento, parte da resolução da equação diferencial regente da instabilidade lateral, para vigas com as mesmas particularidades setoriais das vigas de seção retangular. Entretanto, conforme verificado para a viga de argamassa armada no item 5.3, o estudo de CATANIA \& COCCHI (1985) pode ser utilizado para as análises da instabilidade nas seções duplo $T$ simétrico com bons resultados. Porém, recomenda-se proceder à determinação da carga crítica em seções delgadas duplo $\mathrm{T}$ simétrico pelo programa desenvolvido neste trabalho, para as análises da mudança de equilíbrio, porque considera as características das seções delgadas, bem como os deslocamentos e giros permitidos pela ligação dos apoios.

Quanto ao estudo da instabilidade em vigas pré-moldadas durante a fase de suspensão, o trabalho de CATANIA \& COCCHI (1985) apresenta algumas particularidades e limitações: não consideram os graus de liberdade proporcionados pelas vinculações temporárias e incompletas dos cabos de içamento, e na suspensão com cabos verticais, a carga crítica tende a zero. Por outro lado, anulando a componente 
horizontal da força de içamento, na disposição inclinada dos cabos para a elevação da viga, resulta uma carga crítica equivalente à carga crítica de serviço. Como já referido no capítulo 5, estas inconsistências resultam de um estudo com várias aproximações e considerações.

Enfim, as análises da instabilidade lateral foram baseadas nas hipóteses de regime elástico-linear. Desta forma, a carga crítica neste trabalho abordada representa o limite da instabilidade elástica, a partir do qual ocorre mudança de equilíbrio em teoria de segunda ordem.

No entanto, o estudo elástico é importante por permitir, como uma primeira aproximação, o cálculo da carga crítica de instabilidade lateral. Por outro lado, considerar o comportamento não linear dos materiais bem como a sua relação com o surgimento de fissuras resulta em um estudo mais realista.

O trabalho de KRAUS (1990) considera o comportamento não linear dos materiais, através de um modelo de rigidez que calcula a seção de concreto protendido necessária para suportar o fenômeno da flambagem lateral, justificando a dificuldade de se determinar a rigidez efetiva à torção e flexão lateral, que depende da distribuição das tensões na seção transversal em análise.

$\mathrm{Na}$ continuidade desta pesquisa sugere-se incluir a análise não linear do material no estudo da instabilidade lateral nas fases transitórias e nas fases em serviço, considerando a deformabilidade das ligações, utilizando em linhas gerais, o modelo de rigidez apresentado em KRAUS (1990). 


\section{REFERÊNCIAS BIBLIOGRÁFICAS}

[1] ABRAMOWITZ, M.; SEGUN, I. A. (1968). Handbook of mathematical function: with formulas, grafs, and mathematical tables. New York: Dover, pp.896-897.

[2] ASSOCIAÇÃO BRASILEIRA DA CONSTRUÇÃO INDUSTRIALIZADA (1986). Manual técnico de pré-fabricados de concreto, São Paulo, ABCI, 139p.

[3] ASSOCIAÇÃO BRASILEIRA DE NORMAS TÉCNICAS (1978). NB-1: Projeto e execução de obras de concreto armado. Rio de Janeiro, ABNT.

[4] ASSOCIAÇÃO BRASILEIRA DE NORMAS TÉCNICAS (1985). NBR-9062: Projeto e execução de estruturas de concreto pré-moldado. Rio de Janeiro, ABNT.

[5] BOYCE, W. E.; DIPRIMA, R. C. (1979). Equações diferenciais elementares e problemas de valores de contorno. Guanabara Dois. 3 ed. $587 \mathrm{p}$.

[6] CATANIA, M.; COCCHI, G. M. (1976). La stabilità dell'equilibrio di travi prefabbricate durante le fasi transitorie ed in presenza di imperfezioni ed errori costruttivi. Milano, ITEC/la preffabbricazione, atti del congresso c.t.e. 1976 sulla industrializzazione edilizia siena 12-14 novembre, pp.209-224.

[7] CATANIA, M.; COCCHI, G. M. (1985). La stabilità nelle travi prefabbricate in regime transitorio e di esercizio. Milano, ITEC editrice. $185 \mathrm{p}$.

[8] CUNHA, J. C. (1994). Estabilidade de vigas protendidas e esbeltas durante o içamento e o transporte. In: REUNIÃO DO IBRACON, 36., Porto Alegre. Anais. v. 1, pp.299-311. 
[9] FERREIRA, M. A. (1993). Estudo de deformabilidades de ligações para análise linear em pórticos planos de elementos pré-moldados. Tese de Mestrado EESC-USP.

[10] IMPER, R. R.; LASZLO, G. (1987). Handling and shipping of long span bridge beams. PCI Journal, Nov.-Dec., pp.86-101.

[11] KONCZ, T. (1978). Manual de la construcción prefabricada. Tomo 2. H. Blume Ediciones. Rosario, 17. Madrid-5. 2 ed. pp.56-62.

[12] KRAUS, D.; EHRET, K. -H. (1990). Lateral buckling and second order theory of reinforced and prestressed concrete girders. In: BICANIC, N.; MANG, H. eds. Computer aided analysis and design of concrete structures: Proc. 2nd Int. Conf., Austria, 1990. pp.249-261.

[13] KREYSZIG, E. (1988). Advanced engineering mathematics. 6 ed. pp.1068-1071.

[14] LA NORMATIVA SUI PREFABBRICATI (1984). CNR-10025/84, ITEC - LA PREFABBRICAZIONE a cura della redazione e el C.T.E.

[15] MATILDI, P. et al. (1978). Problemi di satatica delle strutture prefabbricate, Quaderni dei corsi di aggiornamento, n. 5. Milano, CTE. pp.359-387.

[16] MAST, R. F. (1989). Lateral stability of long prestressed concrete beams, Part 1. PCI Journal, Jan.-Feb., pp.34-53.

[17] MAST, R. F. (1993). Lateral stability of long prestressed concrete beams, Part 2. PCI Journal, Jan.-Feb., pp.70-88.

[18] MORI, D. D. (1988). Flexo-torção: Barras com seção transversal aberta e paredes delgadas. São Carlos, EESC-USP. 133p. 
[19] PRESTRESSED CONCRETE INSTITUTE (1992). PCI design handbook.4.ed.

[20] RACHID, M. (1983). Instabilidade de barras de secção delgada. São Carlos, EESC-USP. 119p.

[21] RACHID, M.; MORI, D. D. (1989). Instabilidade: conceitos - aplicação na flambagem por flexão. São Carlos, EESC-USP. 130p.

[22] RACHID, M.; MORI, D. D. (1993). Instabilidade: flambagem de barras de secção delgada por torção e flexão. São Carlos, EESC-USP. 166p. 


\section{ANEXO A}

\section{MÉTODO DE RUNGE-KUTTA}

O método de Runge-Kutta ${ }^{1}$ é um método numérico através do qual pode-se construir a solução de uma dada equação diferencial a partir das condições iniciais. Por ser um procedimento numérico, a solucão obtida é aproximada. Entretanto, o método de Runge-Kutta, dentre as formulações por passos, é amplamento utilizado com sucesso, gerando resultados bastante significativos.

A fórmula de Runge-Kutta clássica é equivalente a uma fórmula de Taylor de cinco termos:

$$
y_{n+1}=y_{n}+h y_{n}^{\prime}+\frac{h^{2}}{2 !} y_{n}^{\prime \prime}+\frac{h^{3}}{3 !} y_{n}^{\prime \prime \prime}+\frac{h^{4}}{4 !} y_{n}^{\prime \prime \prime}
$$

A expressão envolve uma média ponderada de valores de $\mathrm{f}(\mathrm{x}, \mathrm{y})$ tomados em pontos diferentes do intervalo $\mathrm{X}_{\mathrm{n}} \leq \mathrm{X} \leq \mathrm{X}_{\mathrm{n}+1}$. Em um sistema de equações diferenciais de $1^{\text {a }}$ ordem do tipo:

$$
y^{\prime}=f(x, y, z) \text { e } z^{\prime}=g(x, y, z) .
$$

as expressões do método de Runge-Kutta de $4^{\mathrm{a}}$ ordem, onde o erro por truncamento por passo é da ordem de $h^{5}$ são:

$$
\begin{aligned}
y_{n+1}=y_{n}+\frac{1}{6}\left(k_{1}+2 k_{2}+2 k_{3}+k_{4}\right)+O(h)^{5} \\
z_{n+1}=z_{n}+\frac{1}{6}\left(l_{1}+2 l_{2}+2 l_{3}+l_{4}\right)+O(h)^{5}
\end{aligned}
$$

e as quantidades auxiliares à formulação são definidas como segue:

${ }^{1}$ CARL DAVID TOLMÉ RUNGE (1856-1927) e WILHELM KUTTA (1867-1944), famosos matemáticos alemães. 


$$
\begin{gathered}
k_{1}=h f\left(x_{n}, y_{n}, z_{n}\right) \quad l_{1}=h g\left(x_{n}, y_{n}, z_{n}\right) \\
k_{2}=h f\left(x_{n}+\frac{h}{2}, y_{n}+\frac{1}{2} k_{1}, z_{n}+\frac{1}{2} l_{1}\right) \\
l_{2}=h g\left(x_{n}+\frac{h}{2}, y_{n}+\frac{1}{2} k_{1}, z_{n}+\frac{1}{2} l_{1}\right) \\
k_{3}=h f\left(x_{n}+\frac{h}{2}, y_{n}+\frac{1}{2} k_{2}, z_{n}+\frac{1}{2} l_{2}\right) \\
l_{3}=h g\left(x_{n}+\frac{h}{2}, y_{n}+\frac{1}{2} k_{2}, z_{n}+\frac{1}{2} l_{2}\right) \\
k_{4}=h f\left(x_{n}+h, y_{n}+k_{3}, z_{n}+l_{3}\right) \\
l_{4}=h g\left(x_{n}+h, y_{n}+k_{3}, z_{n}+l_{3}\right)
\end{gathered}
$$

De forma direta, para uma equação diferencial de segunda ordem do tipo:

$$
y^{\prime \prime}=f\left(x, y, y^{\prime}\right)
$$

o método de Runge-Kutta pode ser aplicado através das expressões abaixo:

$$
\begin{aligned}
y_{n+1}=y_{n}+h & {\left[y_{n}+\frac{1}{6}\left(k_{1}+k_{2}+k_{3}\right)\right]+O(h)^{5} } \\
y_{n+1}^{\prime} & =y_{n}^{\prime}+\frac{1}{6}\left(k_{1}+2 k_{2}+2 k_{3}+k_{4}\right)
\end{aligned}
$$

e os incrementos:

$$
\begin{aligned}
& k_{1}=h f\left(x_{n}, y_{n}, y_{n}^{\prime}\right) \\
& k_{2}=h f\left(x_{n}+\frac{h}{2}, y_{n}+\frac{h}{2} y_{n}^{\prime}+\frac{h}{8} k_{1}, y_{n}^{\prime}+\frac{k_{1}}{2}\right) \\
& k_{3}=h f\left(x_{n}+\frac{h}{2}, y_{n}+\frac{h}{2} y_{n}^{\prime}+\frac{h}{8} k_{2}, y_{n}^{\prime}+\frac{k_{2}}{2}\right) \\
& k_{4}=h f\left(x_{n}+h, y_{n}+h y_{n}^{\prime}+\frac{h}{2} k_{3}, y_{n}^{\prime}+k_{3}\right)
\end{aligned}
$$

As listagens dos programas desenvolvidos estão anexadas a seguir, para os casos gerais de vigas retangulares e vigas de seção duplo T simétrico suspensas por cabos inclinados. 


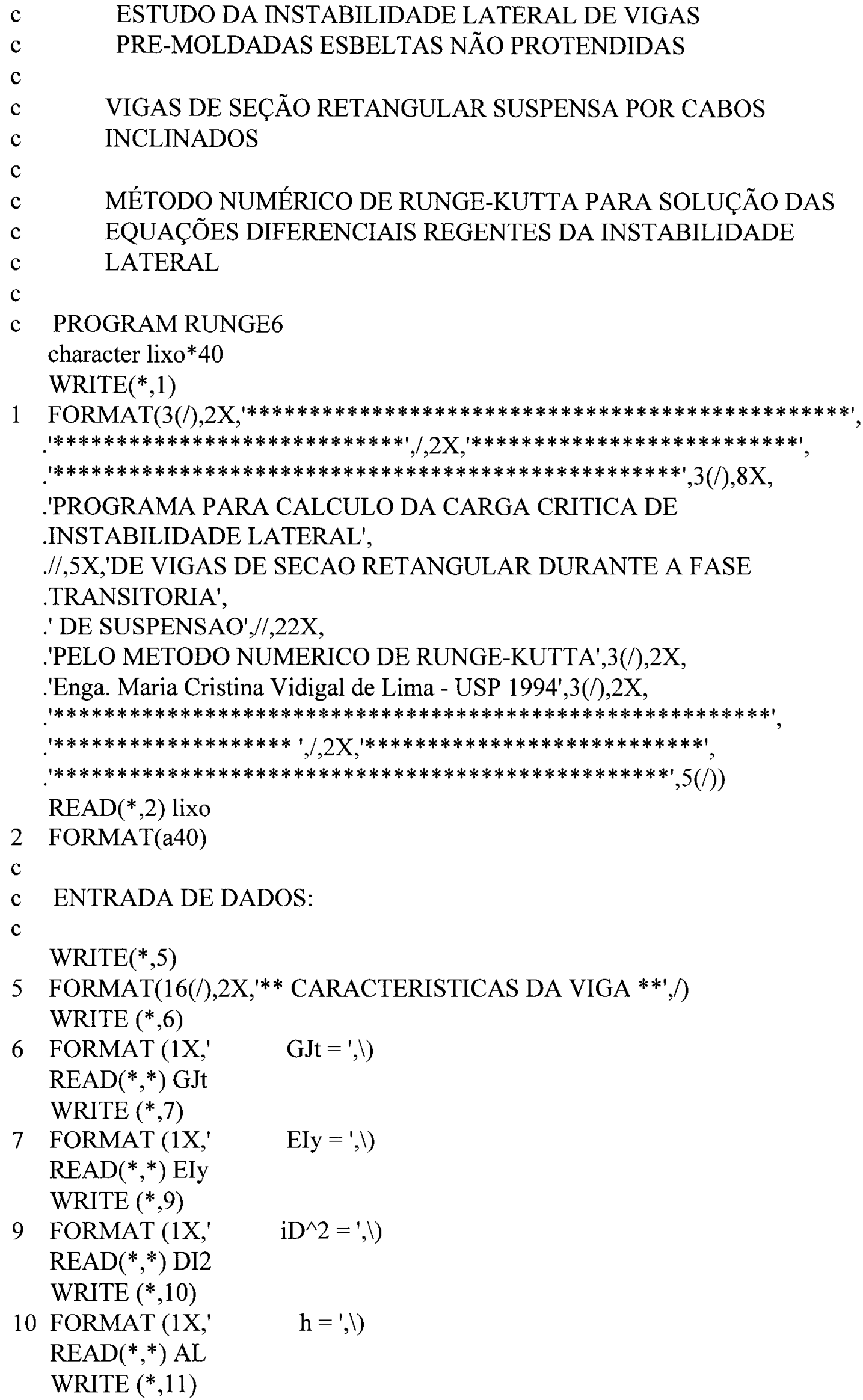




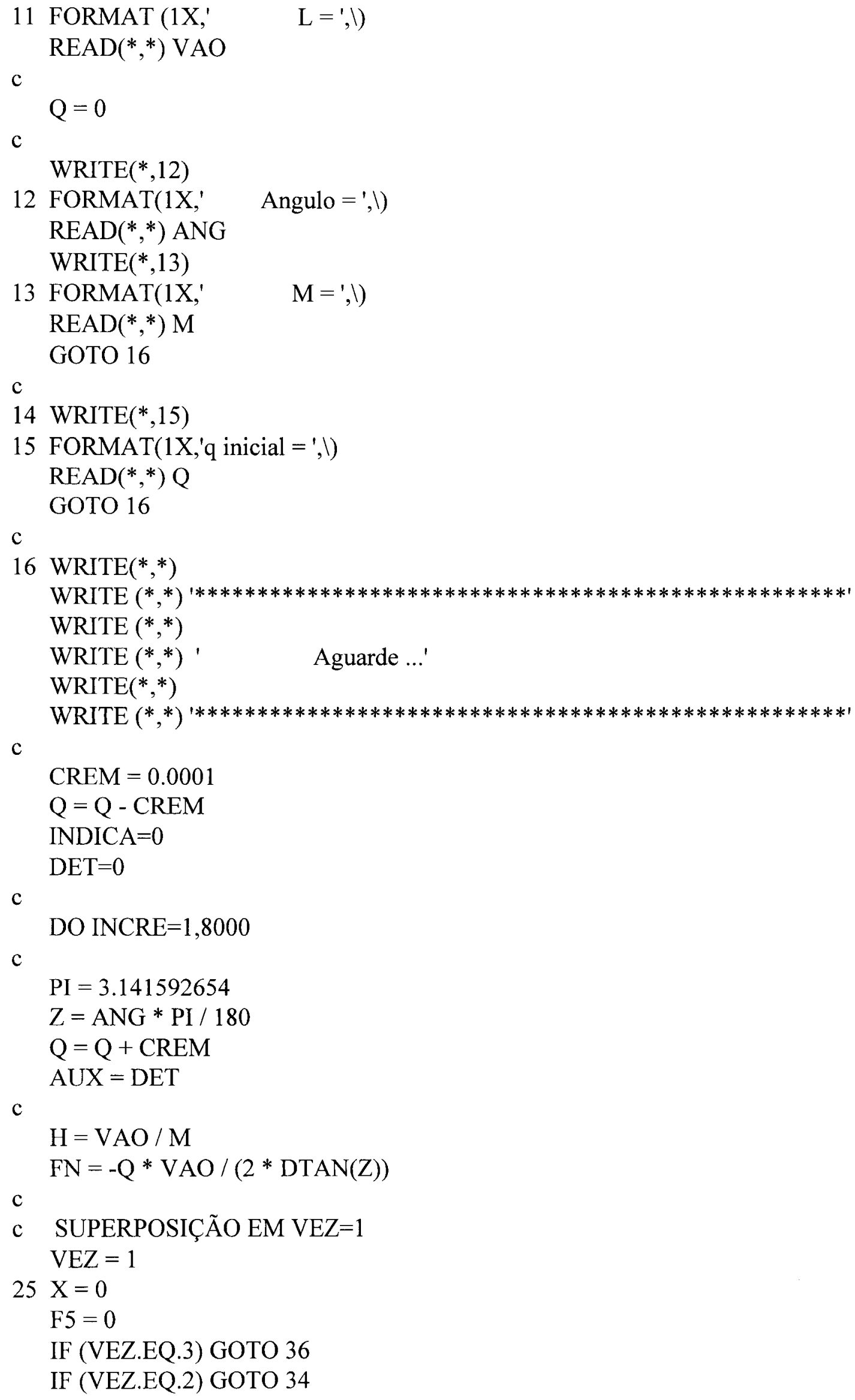




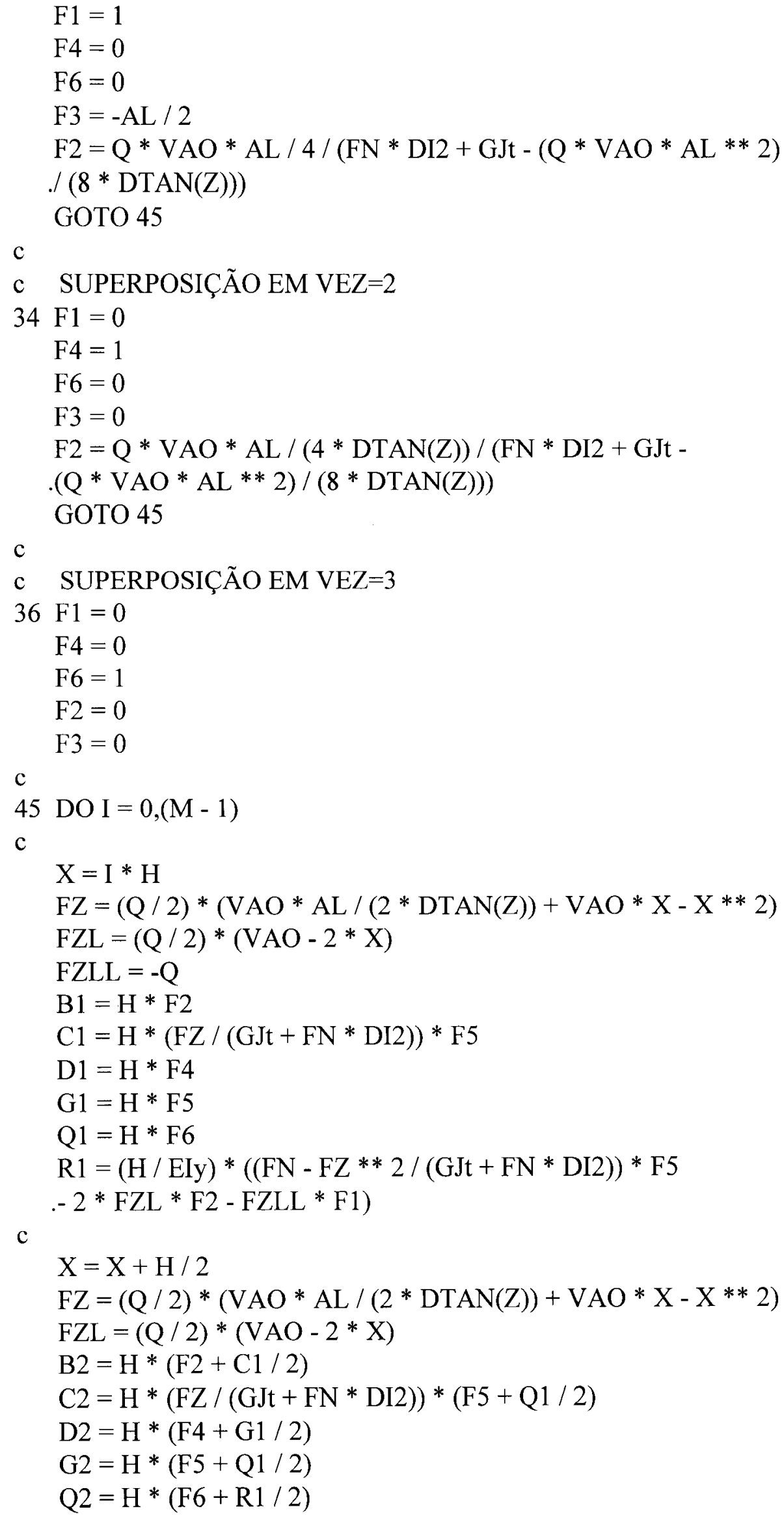




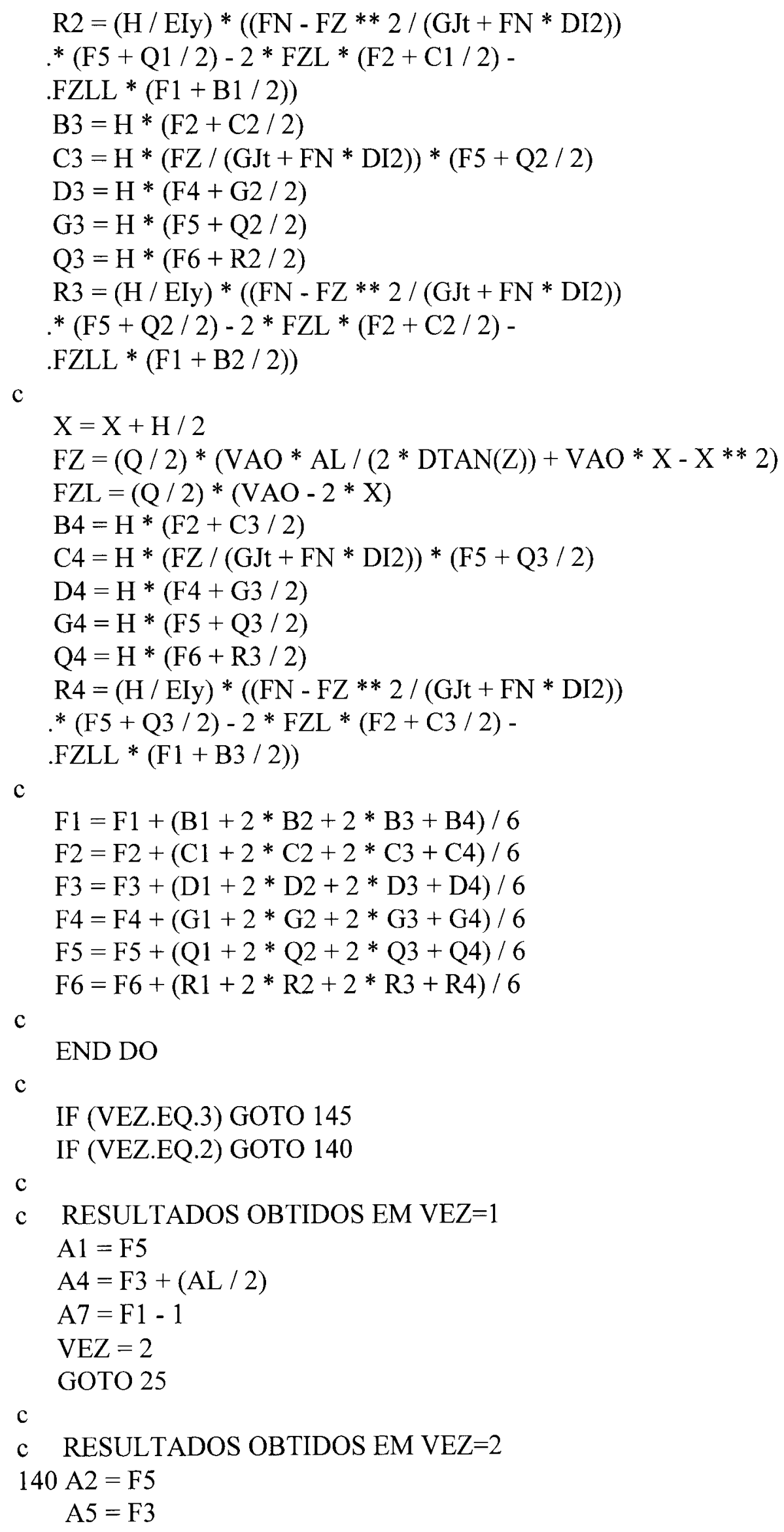




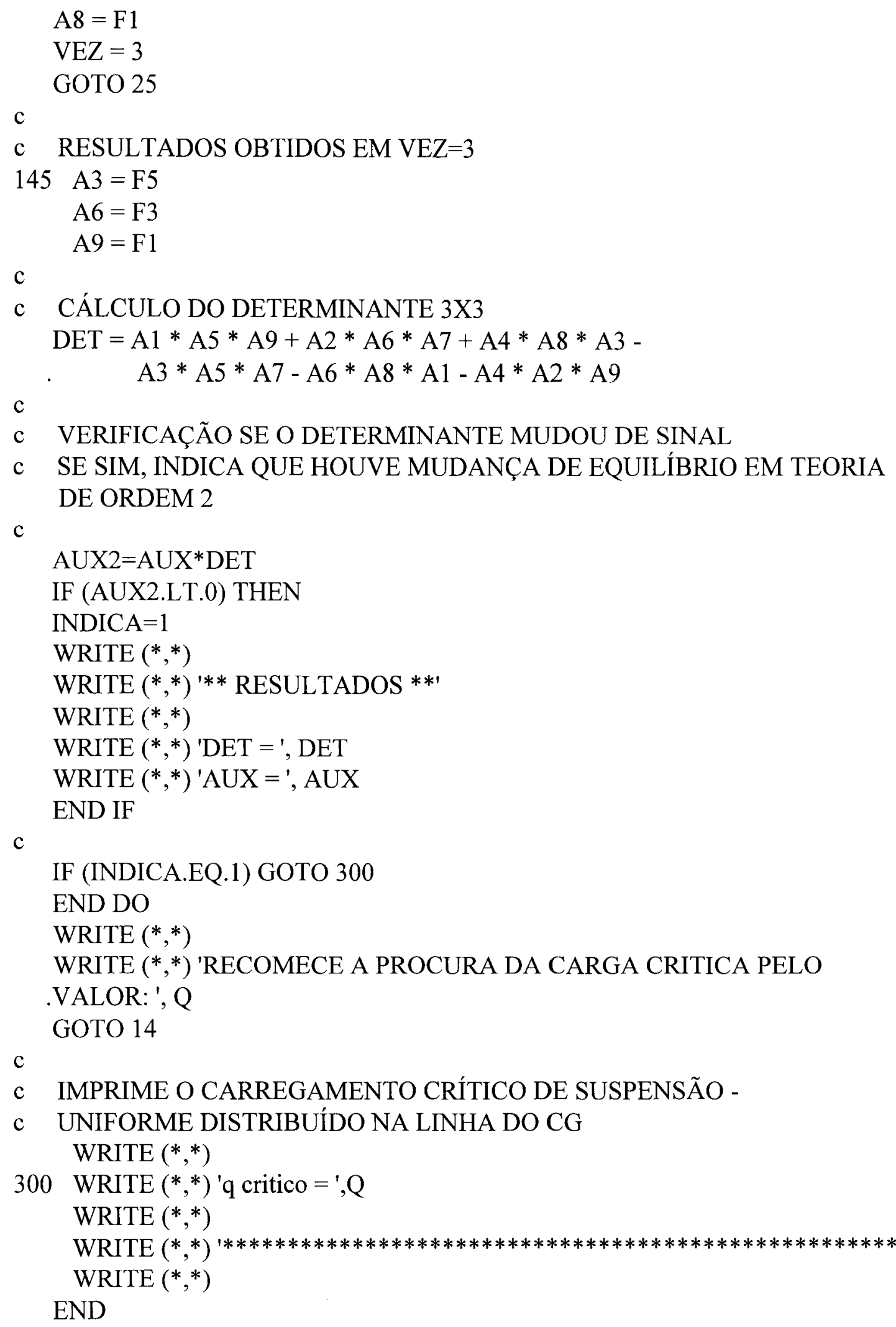




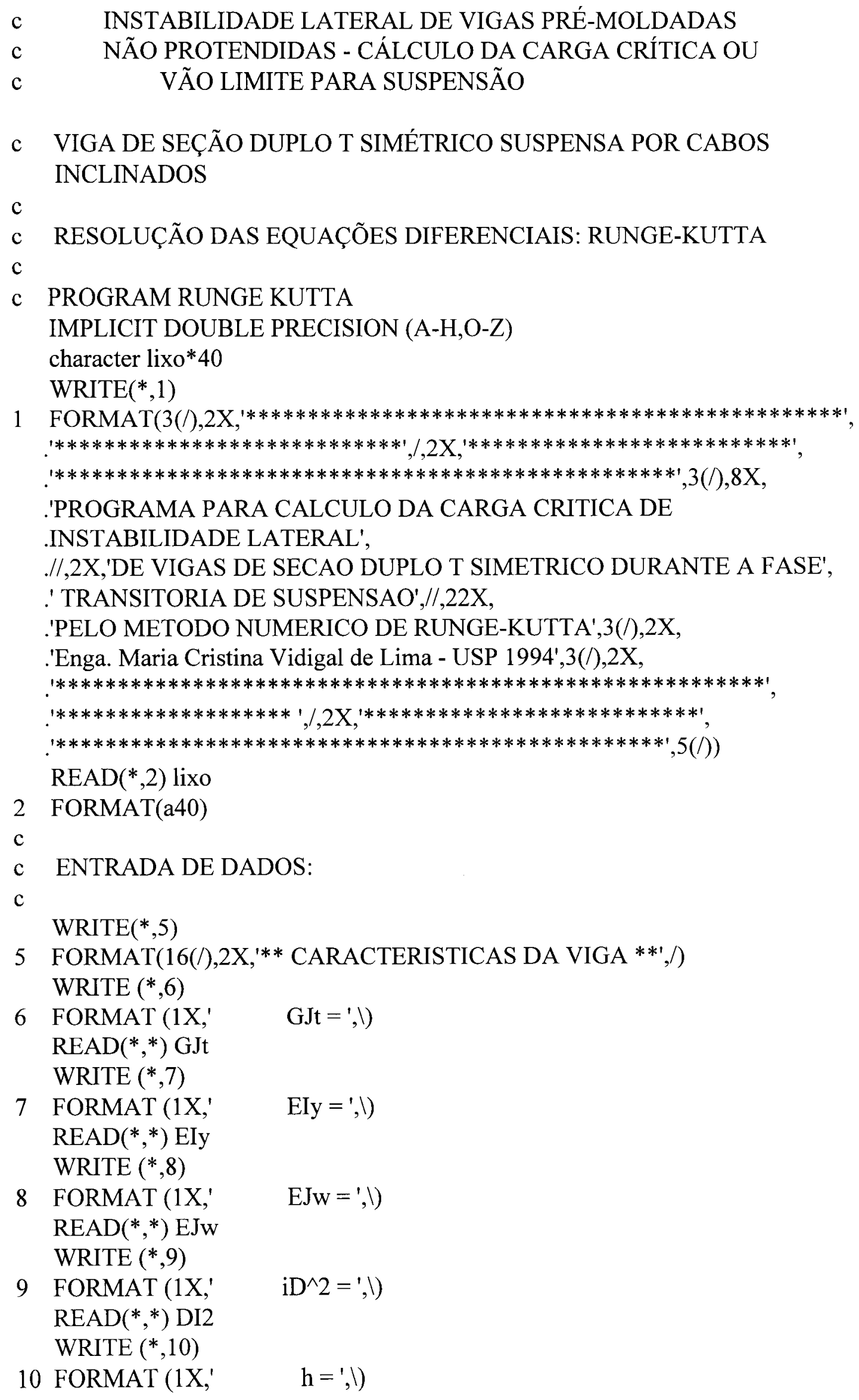




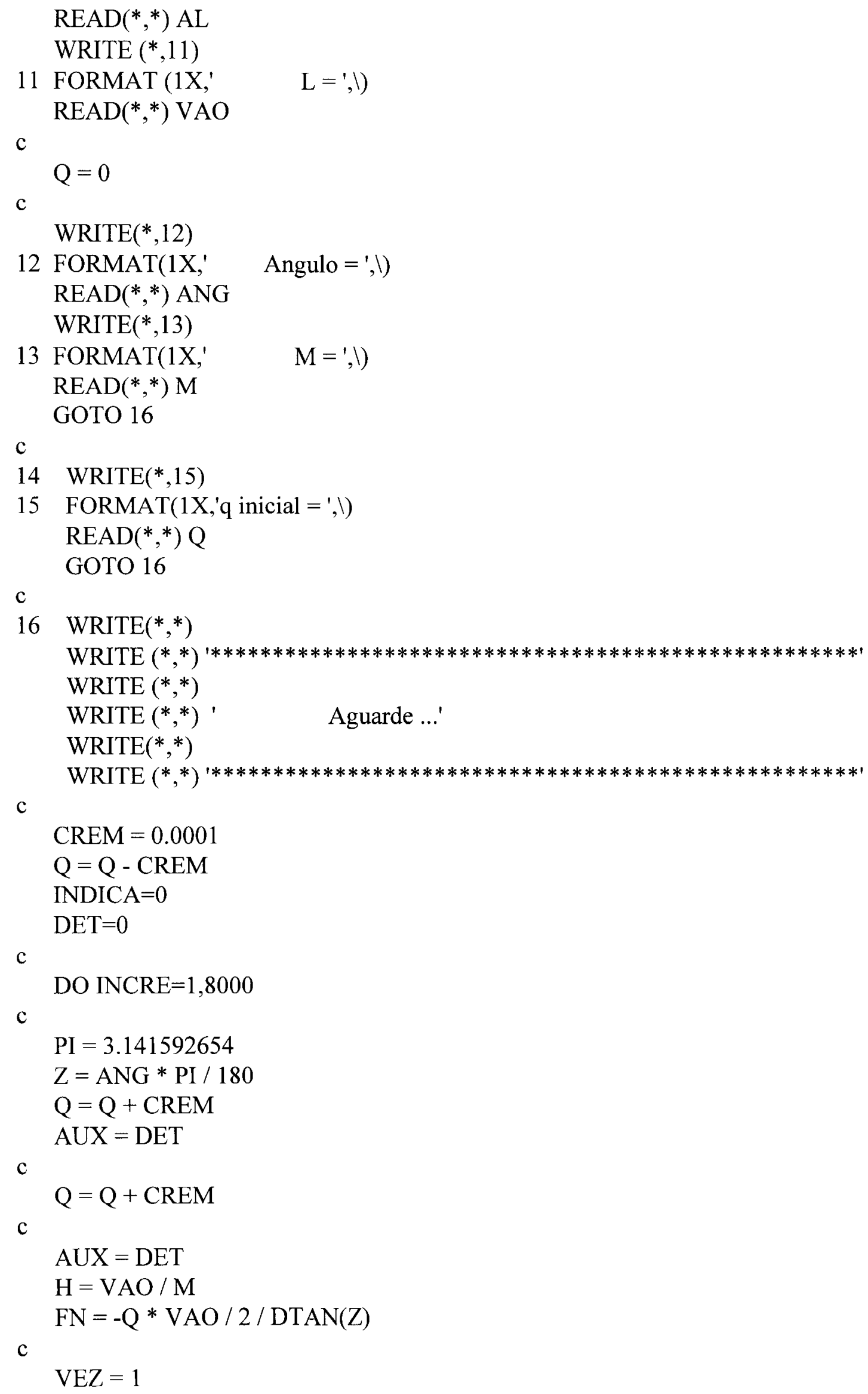




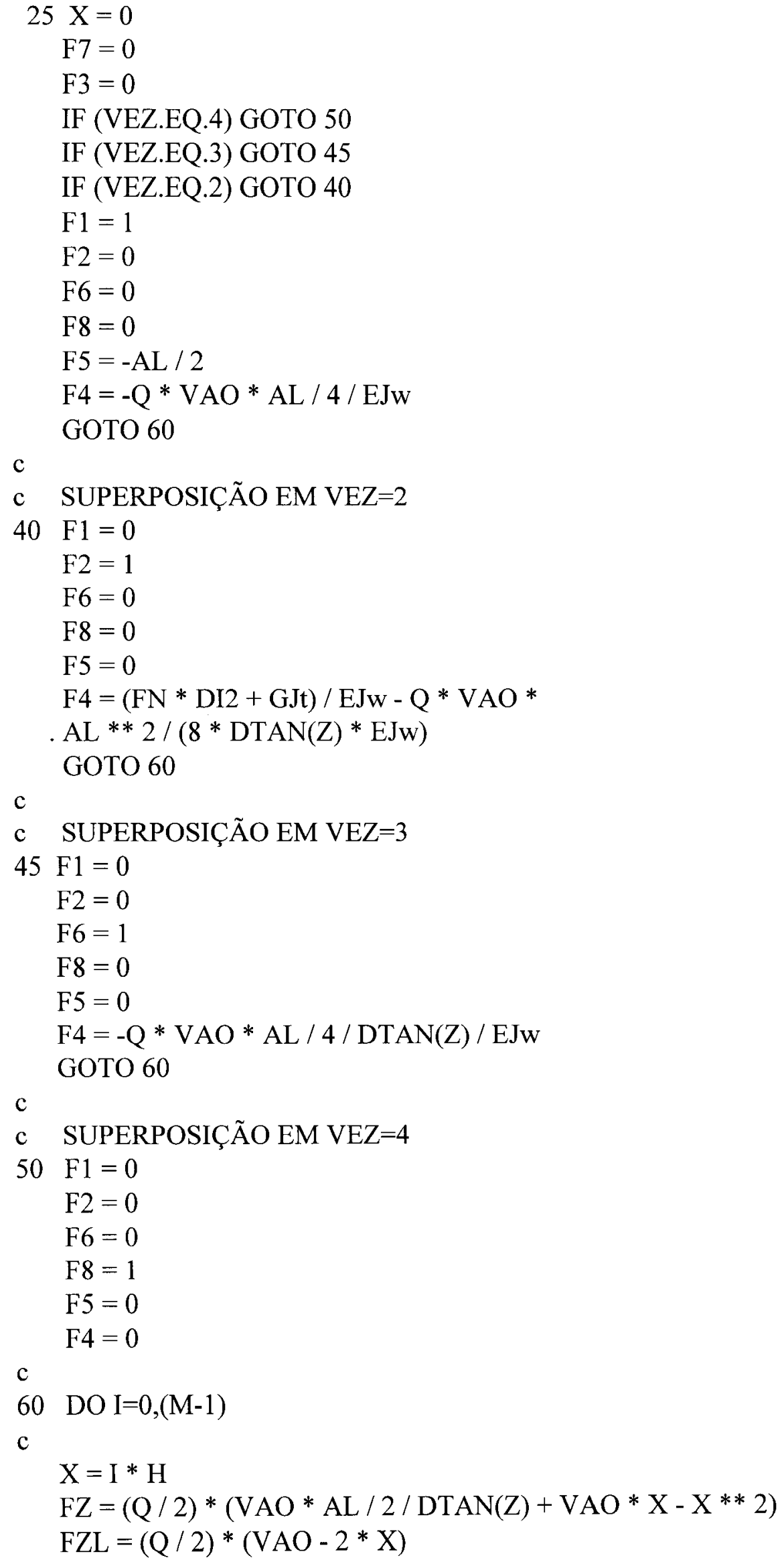




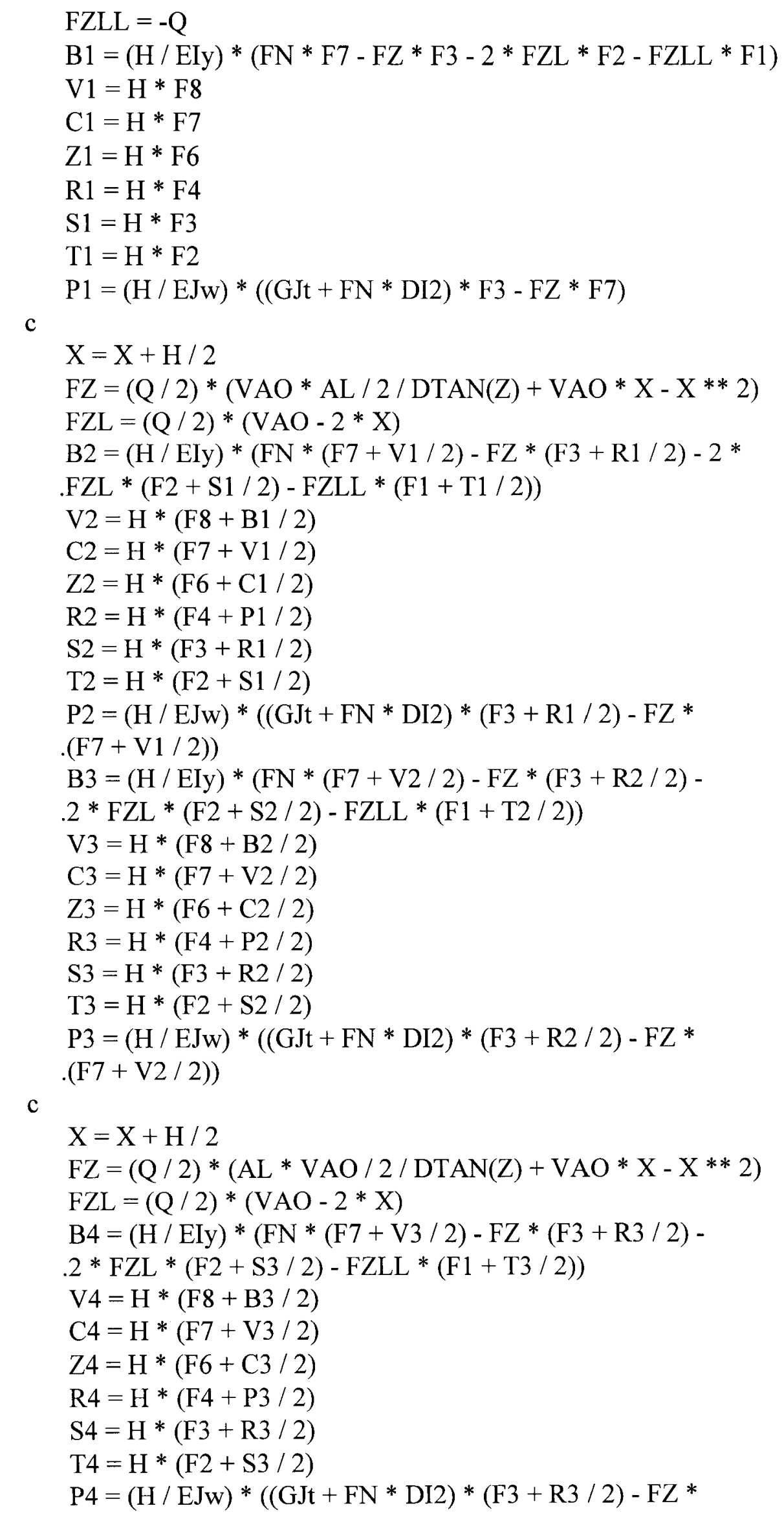




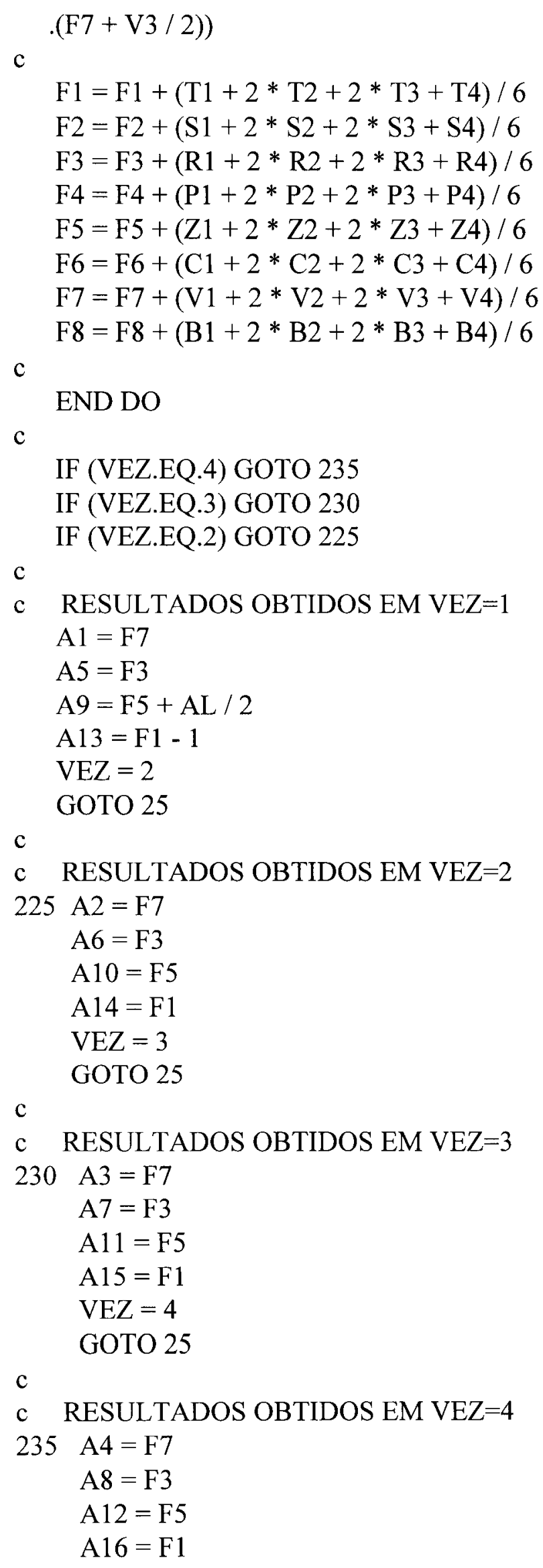


$\mathrm{c}$

c CÁLCULO DO DETERMINANTE $4 \mathrm{X} 4$

$\mathrm{D} 1=\mathrm{A} 6 * \mathrm{~A} 11 * \mathrm{~A} 16+\mathrm{A} 7{ }^{*} \mathrm{~A} 12 * \mathrm{~A} 14+\mathrm{A} 10 * \mathrm{~A} 15 * \mathrm{~A} 8-\mathrm{A} 8 *$

.A11* A14-A $12 * \mathrm{~A} 15 * \mathrm{~A} 6-\mathrm{A} 10 * \mathrm{~A} 7 * \mathrm{~A} 16$

$\mathrm{D} 2=\mathrm{A} 2 * \mathrm{~A} 11 * \mathrm{~A} 16+\mathrm{A} 3 * \mathrm{~A} 12 * \mathrm{~A} 14+\mathrm{A} 10 * \mathrm{~A} 15 * \mathrm{~A} 4-\mathrm{A} 4 *$

$\mathrm{A} 11 * \mathrm{~A} 14-\mathrm{A} 12 * \mathrm{~A} 15 * \mathrm{~A} 2-\mathrm{A} 10 * \mathrm{~A} 3 * \mathrm{~A} 16$

$\mathrm{D} 3=\mathrm{A} 2 * \mathrm{~A} 7 * \mathrm{~A} 16+\mathrm{A} 3 * \mathrm{~A} 8 * \mathrm{~A} 14+\mathrm{A} 6 * \mathrm{~A} 15 * \mathrm{~A} 4-\mathrm{A} 4 * \mathrm{~A} 7 *$

$\mathrm{A} 14-\mathrm{A} 8 * \mathrm{~A} 15 * \mathrm{~A} 2-\mathrm{A} 6 * \mathrm{~A} 3 * \mathrm{~A} 16$

$\mathrm{D} 4=\mathrm{A} 2 * \mathrm{~A} 7 * \mathrm{~A} 12+\mathrm{A} 3 * \mathrm{~A} 8 * \mathrm{~A} 10+\mathrm{A} 6 * \mathrm{~A} 11 * \mathrm{~A} 4-\mathrm{A} 4 * \mathrm{~A} 7 *$

$\mathrm{A} 10-\mathrm{A} 8 * \mathrm{~A} 11 * \mathrm{~A} 2-\mathrm{A} 6 * \mathrm{~A} 3 * \mathrm{~A} 12$

$\mathrm{DET}=\mathrm{A} 1 * \mathrm{D} 1-\mathrm{A} 5 * \mathrm{D} 2+\mathrm{A} 9 * \mathrm{D} 3-\mathrm{A} 13 * \mathrm{D} 4$

c

c VERIFICAÇÃO SE O DETERMINANTE MUDOU DE SINAL

c INDICA MUDANÇA DE EQUILÍBRIO EM TEORIA DE ORDEM 2

$\mathrm{c}$

$\mathrm{AUX} 2=\mathrm{AUX} * \mathrm{DET}$

IF (AUX2.LT.0) THEN

INDICA $=1$

$\operatorname{WRITE}(*, *)$

WRITE $\left(*,{ }^{*}\right)^{\prime * *} \operatorname{RESULTADOS} * *$ '

$\operatorname{WRITE}(*, *)$

WRITE $\left(*,{ }^{*}\right)$ 'DET $=$ ', DET

WRITE $(*, *)$ 'AUX =', AUX

END IF

c

IF (INDICA.EQ.1) GOTO 290

END DO

WRITE $(*, *)$

WRITE $(*, *)$ 'RECOMECE A PROCURA DA CARGA CRITICA PELO

.VALOR: ', Q

GOTO 14

$\mathrm{c}$

c IMPRIME VALOR CRÍTICO

c SE q FOI ASSUMIDO COMO DADO CONHECIDO, IMPRIME Lcrít

c SE L FOI ASSUMIDO COMO DADO CONHECIDO, IMPRIME qcrít

c

$\operatorname{WRITE}(*, *)$

290 WRITE $(*, *)$ 'q critico $=$ ',Q

$\operatorname{WRITE}(*, *)$

$\operatorname{WRITE}(* *)^{\prime *}$

WRITE $(*, *)$

END 